UNITED STATES

DEPARTMENT OF THE INTERIOR

GEOLOGICAL SURVEY

PRELIMINARY DATA FROM A SERIES OF ARTIFICIAL RECHARGE

EXPERIMENTS AT STANTON, TEXAS

By R. L. Bassett, E. P. Weeks, M. L. Ceazan, S. G. Perkins, D. C. Signor,

D. L. Redinger, R. L. Malcolm, G. R. Aiken, E. M. Thurman, P. A. Avery,

W. W. Wood, G. M. Thompson, and G. K. Stiles

Open-File Report $81-149$

Denver, Colorado

1981 
UNITED STATES DEPARTMENT OF THE INTERIOR

JAMES G. WATT, Secretary

GEOLOGICAL SURVEY

Doyle G. Federick, Acting Director

For additional information write to:

U.S. Geologica1 Survey

Water Resources Division

Mail Stop 413, Box 25046

Denver Federal Center

Denver, Colorado 80225

For purchase, write to:

Open File Services Section

U.S. Geologica1 Survey

Box 25425

Denver Federal Center

Denver, Colorado 80225

(303) 234-5888; FTS 234-5888 


\section{CONTENTS}

Table of units, abbreviations, and symbols

Abstract-

Introduction--

Site description and monitoring techniques-_-

Geology-

Hydrology-_-

Analytical methods-_-_-

Genera1-_-

Fluorocarbon-compound analysis-_-

Nonvolatile organic-compound analysis-_-

March 1977 pumping test--

March 1978 injection test-_

Hydraulic data-_-

Flowmeter logs-

Water-level data and analysis-

Sampling schedule and tracer data-_-

Apri1 1978 wel1-redevelopment test-__

May 1978 injection test-_-

Hydraulic data-_-_-_-

Chemical and tracer data--_-

Sampling schedule and tracer data-_

August 1978 injection test--

Hydraulic data--

Sampling schedule and tracer data-

December 1978 tracer test--

References

\section{ILLUSTRATIONS}

Figure 1. Location map of the Stanton, Tex. experimenta1 site on the Southern High Plains of Texas-

2. Plan view of the arrangement of wells and physical plant at the Stanton, Tex. research site--

3. Schematic showing construction of the point samplers used in this investigation-

4. Fence diagram showing the location of point samplers, observation wells, and piezometers at the Stanton,

5ex. research site- Cross section of the point-sampler arrangement, and resistivity $\log$ for the saturated part of the aquifer---

6. Sketch of the bypass chamber used to produce a virtually instantaneous pulse of tracer-tagged water in the injection stream- 
Figure 7. Lithologic column from the Stanton, Tex. site with point locations of mineralogical analyses, porosity, and hydraulic-conductivity determinations--

8. Sketch of stripping, concentration, and analytical system for fluorocarbons in the nanogram per liter range-----

9-12. Graphs showing:

9. Variation of input concentration with time of the fluorocarbon bromochlorodifluoromethane during the first tracer experiment, August 8, 1978--

10. Variation of input concentration with time of the fluorocarbon dichlorodifluoromethane during the second tracer experiment, August 10-11, 1978-

11. Variation of input concentration with time of the fluorocarbon dibromodifluoromethane during the third tracer experiment, August 12-13 12, 1978---------

12. Flowmeter logs from the injection well obtained during the March 1978 test--_-_-_-

13. Flowmeter logs from the injection well obtained during the May 1978 test--_-

14-38. Graph showing:

14. Change in specific conductance measured in samples from the 2-meter well and point samplers collected May $24-25,1978$

15. Change in specific conductance measured in samples from the 5-meter well and point samplers collected May 24-25, 1978-

16. Change in alkalinity measured in samples from the 2-meter well and point samplers collected May $24-25,1978$

Change in alkalinity measured in samples from the 5 -meter well and point samplers collected May 24-25, 1978-

18. Flowmeter logs from the injection well obtained during the August 7-15, 1978 test--

19. Bromide data obtained from the 2-meter well and point samplers during the first experiment of the August 1978 test--

20. Bromide data obtained from the 5-meter well and point samplers during the first experiment of the August 1978 test--

21. Bromide data obtained from the 10- and 15-meter wells during the first experiment of the August 1978 test----

22. Bromide data obtained from the 2-, 5-, 10-, and 15-meter wells during the first experiment of the August 1978 test-_-_- 


\section{ILLUSTRATIONS--Continued}

Figure 23. Boron data obtained from the 2-meter well and point samplers during the first experiment of the August 1978 test-_-_-

24. Boron data obtained from the 5-meter well and point
samplers during the first experiment of the

24. Boron data obtained from the 5-meter well and
samplers during the first experiment of the August 1978 test----

25. Boron data obtained from the 10- and 15-meter wells during the first experiment of the August 1978 test---------

26. Boron data obtained from the 30 -meter well and point samplers during the first experiment of the August 1978 test--

27. Boron data obtained from the 2-, 5-, 10-, 15-, and 30-meter wells during the first experiment of the August 1978 test-----

28. Comparison of boron, bromide, and bromochlorodifluoromethane data obtained from the 2-meter well during the first experiment of the August 1978 test-----

29. Comparison of boron, bromide, and bromochlorodifluoromethane data obtained from the 5-meter well during the first experiment of the August 1978 test-----

30. Comparison of tracer data obtained from the 2-meter well during the second experiment of the August 1978 test----

31. Comparison of bromide, benzoate, and phenolphthalein data obtained from the 2-meter orange point sampler during the second experiment of the August 1978 test--

32. Comparison of tracer data from the 5-meter orange point sampler during the second experiment of the August 1978 test--

33. Comparison of tracer data from the 2-meter orange point sampler during the third experiment of the August 1978 test---

34. Comparison of tracer data from the 5-meter orange point sampler during the third experiment of the August 1978 test--

35. Comparison of halide tracer data with yeast from the 2-meter well during the fourth experiment (pulse injection) of the August 1978 test--

36. Comparison of halide tracer data obtained from the 5-meter well during the fourth experiment (pulse injection) of the August 1978 test---_---

37. Comparison of halide and yeast tracer data from the 5-meter red piezometer during the fourth experiment pulse injection) of the August 1978 test---

38. Comparison of halide tracer data obtained from the 5-meter orange point sampler during the fourth experiment (pulse injection) of the August 1978 test---- 
Table 1. Summary of the tracers used in the four geochemical tests-_- 4

2. Depth and type of sampling locations-_-_-_-_-_-_--- 11

3. Mineralogy of the lithologic column as determined from cores taken from the area adjacent to the injection well-- - - - - - - -

4. Mineralogy of the heavy minerals present in the cores obtained from an area adjacent to the injection well

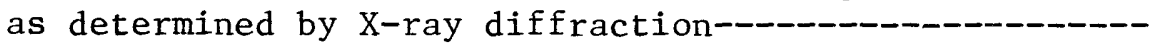
orosity and hydraulic-conductivity data determined from core samples from an area adjacent to the injection

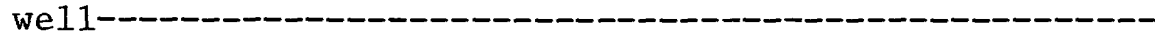

- Transmissivity and storage values computed for the Oga1lala aquifer based on data from the March 1977 pumping test---------

7. Tracer information for March 1978 injection test---------

8. Injection rates and cumulative volumes for the

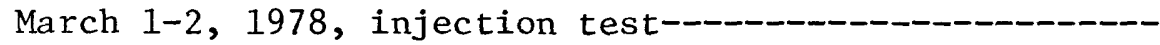

9. Transmissivity and storage values computed for the Ogallala aquifer based on data from the March 1978 injection test-----

10-21. Data obtained from the first tracer experiment on March 1, 1978, at the Stanton, Tex. site

10. 2-meter well-_-_-

11. 2-meter black point sampler-_-

12. 2-meter red point sampler-

13. 2-meter orange point sampler-_-

14. 2-meter green point sampler--

15. 5-meter wel1-_-_- 47

16. 5-meter black point sampler-- 49

17. 5-meter orange point sampler-- 50

18. 5-meter green point sampler-- 52

19. 10-meter wel1-- 53

20. 15-meter we11-_- 54

21. 30-meter we11-_-_- 55

22-29. Data obtained from the second tracer experiment on March 1, 1978, at the Stanton, Tex. site

22. 2-meter we11-_- 56

23. 2-meter black point sampler--_-_- 58

24. 2-meter red point sampler-- 59

25. 2-meter orange point sampler-_- 61

26. 2-meter green point sampler-_-_-_-_- 63

27. 5-meter we11-_- 64

28. 5-meter black point sampler-_- 65

29. 5-meter orange point sampler-_- 66 
TABLES--Continued

Table 30. Injection rates and amounts; Stanton injection test, May 24-25, 1978--

Page

31. Analyses of the playa-lake water and ground water prior to the May 24-25, 1978, injection test----

32. Tracer information for May injection---

33-43. Data obtained from water samples collected during the May 24-25, 1978, injection test using playa-lake water

33. 2-meter we11-

34. 2-meter black point sampler--

35. 2-meter red point sampler-

36. 2-meter orange point sampler--

37. 2-meter green point sampler-

38. 5-meter wel1-_-_-

39. 5-meter black point sampler-

40. 5-meter red point sampler-

41. 5-meter orange point sampler--

42. 10-meter point sampler--

43. 15-meter point sampler--

44. Cumulative volume of water injected after selected times during the August 1978 injection test----

45. Tracer information for the August 1978 test--

46. Analysis of the ground water used during the August 1978 tracer test--

47-60. Data obtained from the tracer experiments conducted during August 8-15, 1978, at the Stanton, Tex. site

47. 2-meter we11--

48. 2-meter black point sampler-_-

49. 2-meter red point sampler--

50. 2-meter orange point sampler-

51. 5-meter we11--

52. 5-meter black point sampler--

53. 5-meter red point sampler-_-

54. 5-meter orange point sampler-_-

55. 10-meter we11-_-

56. 15-meter we11-

57. 30-meter wel1--

58. 30-meter black point sampler--

59. 30-meter red point sampler--

60. 30-meter orange point sampler-

61-66. Comparison between all tracers used during the second tracer experiment written in terms of the unitless concentration ratio $\mathrm{C} / \mathrm{Co}$

61. 2-meter wel1-

62. 2-meter red point sampler--

63. 2-meter orange point sampler- 177 
Table 64. 5-meter we11-_- 65.

Page

65. 5-meter orange point sampler-

179

66. 15-meter we11- point sampler- 181

67-74. Comparison between all tracers used during the third tracer experiment written in terms of the unitless concentration ratio $\mathrm{C} / \mathrm{Co}$

67. 2-meter wel1

68. 2-meter red point sampler-_-_-_-_-_-_-_-_-_-_-_-_- 185

69. 2-meter orange point sampler- 187

70. 5-meter we11- 189

71. 5-meter red point wel1-_- 191

72. 5-meter orange point sampler- 193

73. 10-meter wel1-- 195

74. 15-meter we11-- 196

75-82. Comparison between all tracers used during the fourth tracer experiment (began 1200, August 14, 1978) and the fifth tracer experiment (began 1000, August 15, 1978)

75. 2-meter wel1--

76. 2-meter black point sampler-_- 199

77. 2-meter red point sampler--_- 200

78. 2-meter orange point sampler-_- 202

79. 5-meter well-_- 203

80. 5-meter red point sampler-_- 205

81. 5-meter orange point sampler- 207

82. 5-meter black point sampler-a 209

83. Injection rates and cumulative volumes for the December 1978 two-we11 tracer test-- 230

84. Chemical data from the two-we11 tracer test conducted
during December 1978 at the Stanton, Tex. site----- 232 
TABLE OF UNITS, ABBREVIATIONS, AND SYMBOLS

Units and Abbreviations

C/Co Unitless ratio of measured concentration divided by the initial concentration

d Day

h Hour

$\mathrm{kg} \quad$ Kilogram

$\mathrm{kW} \quad$ Kilowatt

L Liter

L/s Liter per second

$\mathrm{m} \quad$ Meter

$\mathrm{m}^{3} \quad$ Cubic meter

$\mathrm{m}^{2} / \mathrm{d} \quad$ Meter squared per day

$\mathrm{m} / \mathrm{d} \quad$ Meter per day

$\mathrm{m} / \mathrm{km} \quad$ Meter per kilometer

$\mathrm{mg} / \mathrm{L} \quad$ Milligram per liter

$\mathrm{mg} C / \mathrm{L} \quad$ Milligram of carbon per liter

min Minute

$\mathrm{mL} \quad$ Milliliter

$\mathrm{mm} \quad$ Millimeter

$\mathrm{nm} \quad$ Nanometer

ng/L Nanogram per liter

$\mathrm{Pa} \quad$ Pascal

TNTC Too numerous to count

$\mu \mathrm{g} / \mathrm{L} \quad$ Microgram per liter

$\mu \mathrm{L} \quad$ Microliter

\section{Symbols}

$\mathrm{BCF}, \mathrm{CBrClF} 2$ Bromochlorodifluoromethane

DBM, $\mathrm{CBr}_{2} \mathrm{~F}_{2}{ }^{2}$ Dibromodifluoromethane

DOC 22 Dissolved organic carbon

F-11, Freon-11 Trichlorofluoromethane

F-12, Freon-12,

and $\mathrm{CCl}_{2} \mathrm{~F}_{2}$ Dichlorodifluoromethane 


\title{
PRELIMINARY DATA FROM A SERIES OF ARTIFICIAL RECHARGE EXPERIMENTS AT STANTON, TEXAS
}

\section{By}

\author{
R. L. Bassett, E. P. Weeks, M. L. Ceazan, S. G. Perkins, \\ D. C. Signor, D. L. Redinger, R. L. Malcolm, G. R. Aiken, \\ E. M. Thurman, P. A. Avery, W. W. Wood, G. M. Thompson, \\ and G. K. Stiles
}

\begin{abstract}
A series of artificial recharge experiments was conducted by the U.S. Geological Survey at an experimental site located in Stanton, Texas. Five tests were performed from March 1977 through December 1978 to: (1) Evaluate the hydraulic properties of the aquifer; (2) test sampling and monitoring equipment; (3) compare tracers for future use in hydrologic investigations; and (4) determine the radial and vertical distribution of hydraulic properties at the site. Suites of inorganic, and both volatile and nonvolatile organic tracers were used in the tests, and comparative data were obtained from sampling points at several radial distances and depths from the injection well. Hydraulic data from aquifer tests and geologic data from core material also were obtained during the investigation.
\end{abstract}

\section{INTRODUCTION}

The U.S. Geological Survey has conducted a series of artificial-recharge experiments at several locations in the Southern High Plains of Texas (Brown and others, 1978). Artificial recharge is defined in this report as a process by which man adds water to an aquifer. This ongoing-research effort is directed toward defining the hydrologic and geochemical characteristics affecting the movement and quality of water that has been added to an aquifer.

A series of five tests was conducted from March 1977 through December 1978 at an experimental facility located within the city limits of Stanton, Tex. (fig. 1). The investigation, which consisted of both hydrologic and geochemical studies, began with a pumping test, followed by three single-well tracer-injection tests, and concluded with a two-well tracer test.

Data from these experiments are discussed in the following sections in chronological order and are referred to as: (1) March 1977 pumping test; (2) March 1978 injection test; (3) May 1978 injection test; (4) August 1978 injection test; and (5) December two-well test. The pumping test was performed to identify the hydraulic properties of the aquifer and to determine 


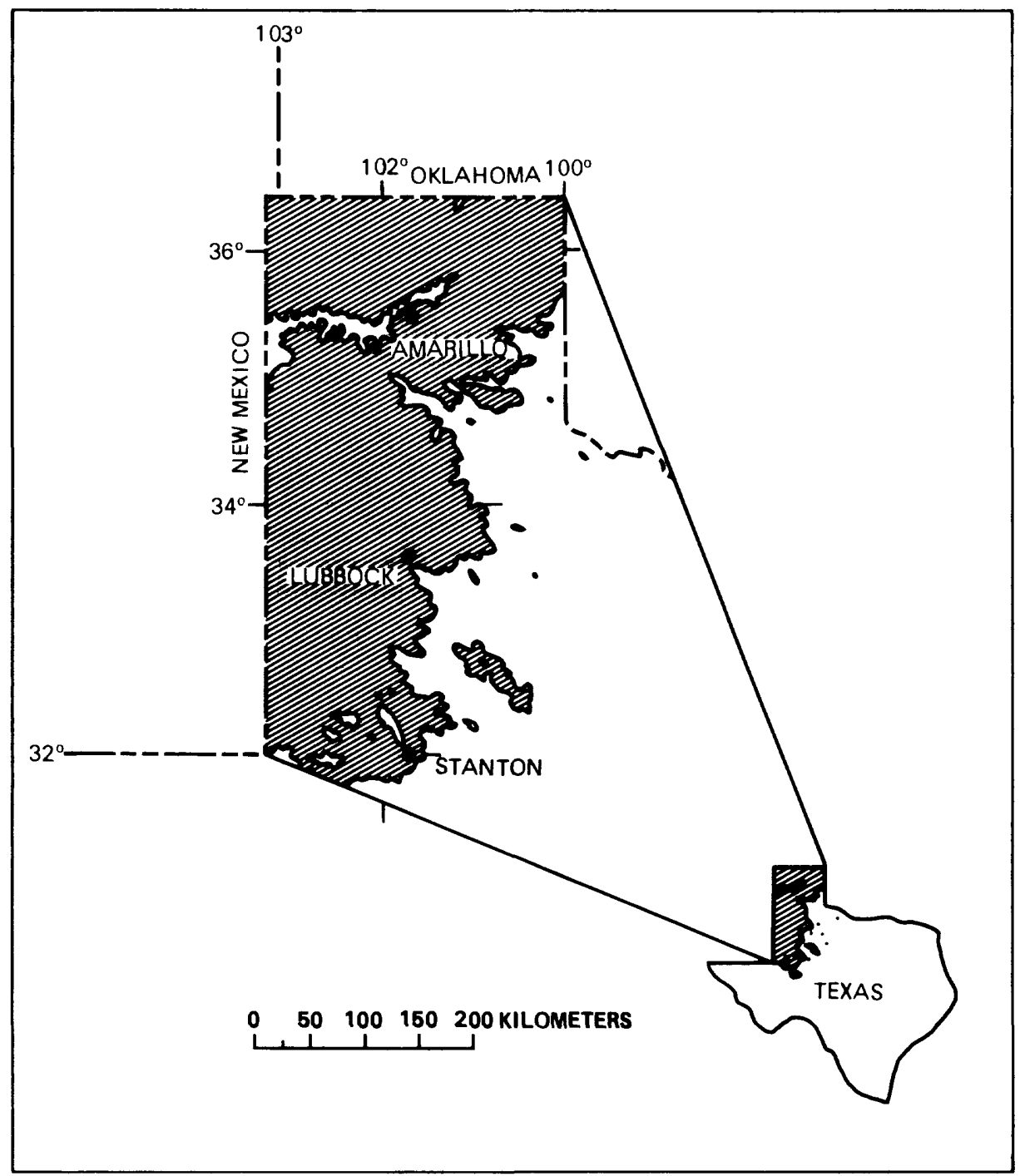

Figure 1.--Location of the Stanton, Tex. experimental site on the Southern High Plains of Texas. 
well loss and efficiency of the injection well. The tracer-injection experiments had the following overall objectives: (1) Testing water-sampling apparatus and monitoring equipment for future investigations; (2) evaluating retention mechanisms for several ground-water tracers; and (3) determining vertical and radial velocity distributions and dispersion coefficients.

This report describes the sampling and monitoring equipment and presents data collected during the five tests. The report deals primarily with the performance of numerous tracers in an unconfined sand-and-gravel aquifer containing argillaceous and carbonaceous material. Only a few selected references by the authors on the tracers used in the Stanton experiments are listed below, as an extensive review of the literature on ground-water tracers is beyond the scope of this report. Davis and others (1980) present a review of commonly used ground-water tracers, as well as some preliminary data on fluorocarbon tracers. Thompson and Hays (1979) describe the use of trichlorofluoromethane $(F-11)$ as an environmental tracer of ground-water movement. Wood and Ehrlich (1978) describe the use of yeast as a particulate tracer of ground-water movement for a previous test on well 1 at the Stanton test site. Dissolved organic carbon (DOC) has been used as an index of organic-contaminant movement from point sources into the ground water (Hughes and others, 1974). The differential movement and persistence of several organic solutes were determined useful in evaluating geochemical aspects of deep-waste storage by Leenheer and Malcolm (1976).

In each of the injection tests, both inorganic and organic compounds were used as tracers; the resultant data are presented in subsequent sections of the report. The operation of the research facility, the planning of the injection tests, and the selection of the inorganic tracers, were the responsibility of the U.S. Geological Survey Artificial-Recharge Project. Dr. Glenn Thompson of the University of Arizona was invited to cooperate in the tests because of his expertise in the analysis of volatile organics (fluorocarbons) used as ground-water tracers. Technical assistance in tracing and analyzing the movement of nonvolatile organic substances in the ground-water system was provided by the U.S. Geological Survey research project on organic geochemistry. Each research group had the responsibility of selecting, administering, and analyzing a group of tracers suitable for this geohydrologic environment. Tracers used in the geochemical studies are itemized in table 1; however, discussion of their analysis and the rationale for the selection, sequencing, and monitoring are deferred to the section on Analytical Methods, and to discussions of the individual tests.

\section{SITE DESCRIPTION AND MONITORING TECHNIQUES}

The Stanton, Texas experimental-recharge facility consists of a deepened and enlarged playa lake, a water-treatment plant, four dual-purpose production-injection we11s, an injection well, and numerous observation wells, piezometers, and water-quality samplers (fig. 2). The lake-storage facilities, treatment plant, and production-injection wells were constructed by the city of Stanton during 1968, using a grant from the U.S. Department of 
Table 1.--Summary of the tracers used in the

four geochemical tests

March 1978: Injection Test

\begin{tabular}{lc}
\hline Experiment 1 & Experiment 2 \\
\hline iodide & ethano1 \\
ethanol & benzoate \\
$\mathrm{CBrClF}_{2}$ & Freon-12 \\
\hline
\end{tabular}

May 1978: Injection Test

natural dissolved organic material

bromide

boron

Freon-12

$\mathrm{CBrClF}_{2}$

$\mathrm{CBr}_{2} \mathrm{~F}_{2}$

August 1978: Injection Test

\begin{tabular}{lllll}
\hline Experiment 1 & Experiment 2 & Experiment 3 & Experiment 4 & Experiment 5 \\
\hline boron & bromide & boron & bromide & Freon-12 \\
bromide & ethanol & ethanol & chloride & Freon-11 \\
copper & acetate & aniline & iodide & $\mathrm{CBrClF}_{2}$ \\
deuterium & phenolphthalein & $\mathrm{CBr}_{2} \mathrm{~F}_{2}$ & fluoride & \\
$\mathrm{CBrClF}_{2}$ & benzoate & ethylamine & yeast & \\
& Freon-12 & & & \\
& & & & \\
\hline
\end{tabular}

December 1978: Two Well Test

benzoate 


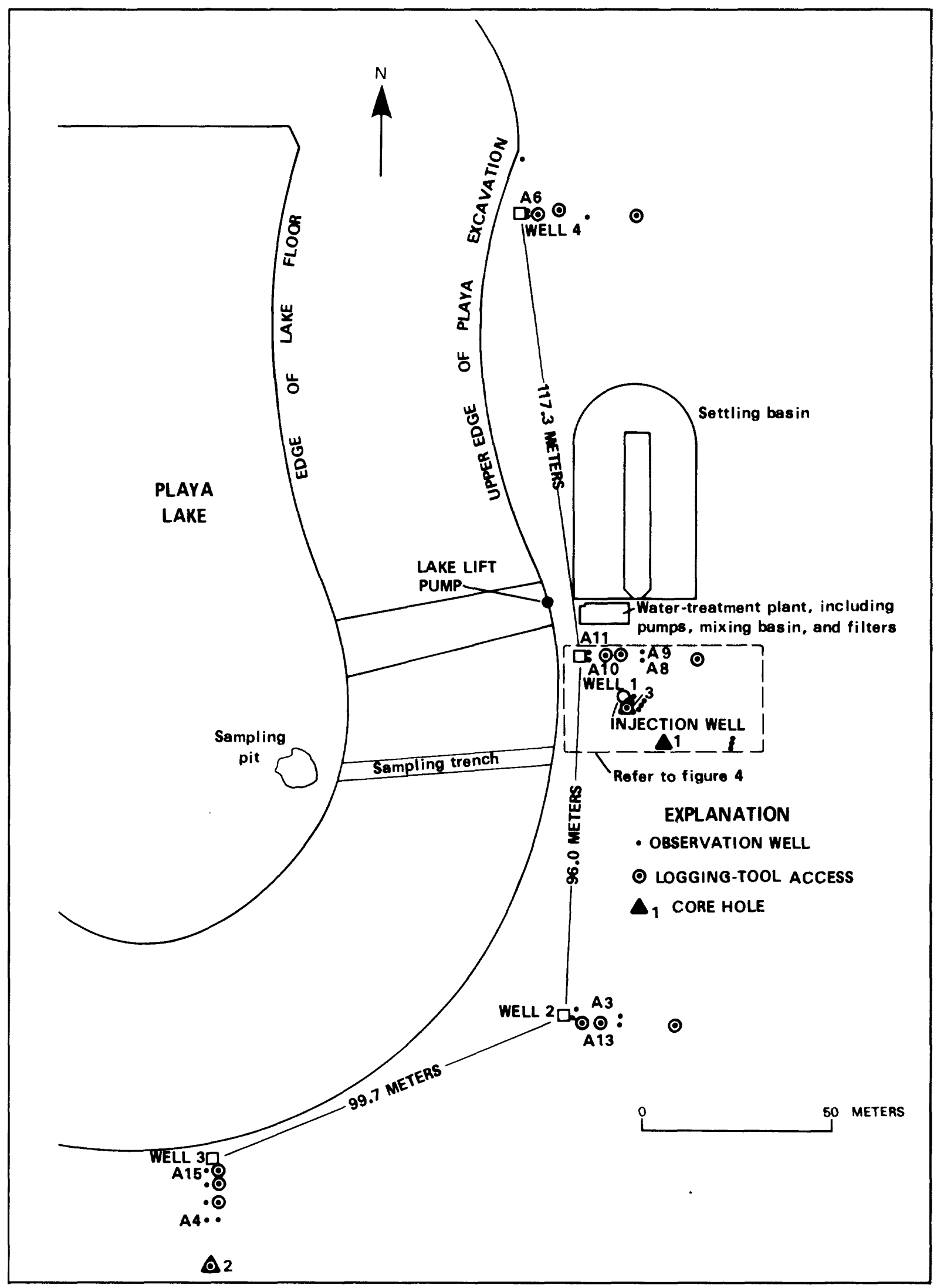

Figure 2.--Plan view of the arrangement of wells and physical plant at the Stanton, Tex. research site. 
Housing and Urban Development. The playa was deepened and enlarged to alleviate periodic flooding of the town. The well systems were constructed to provide a municipal ground-water supply by treating and artificially recharging water that accumulated in the playa for later withdrawal. The High Plains Artificial-Recharge Research Project of the U.S. Geological Survey requested use of the facility for artificial-recharge experiments; this use was granted for 5 years (starting in 1970), and was later extended for another 5 years.

Storage and treatment facilities include the enlarged playa and associated stormwater collection system that drains into the lake; the 1akelift pump to move water from the lake to the treatment facility; chemicalfeed pumps and a mixing basin for adding and mixing chemical flocculating agents; horseshoe-shaped settling basin; pick-up pump for removing water from the horseshoe basin; and three diatomaceous-earth filters (fig. 2). Ground-water and recharge pumpage facilities consist of four wells (fig. 2), drilled through the Ogallala Formation to the underlying Triassic red beds. These wells were drilled to a diameter of $0.75 \mathrm{~m}$ and were cased with $0.25-\mathrm{m}$ casing, slotted from a depth of $21.3 \mathrm{~m}$ to total depth. The wells were grave1-packed to within $12.2 \mathrm{~m}$ of the land surface, and were cemented above that depth. Each well was equipped with a turbine pump powered by a 22.7-kW electric motor. Dual lines connect each well to the treatment plant, including a discharge line for pumping ground water, and a recharge line to transport water from the playa.

The U.S. Geological Survey drilled 17 observation wells and 13 loggingtool access holes within the area (fig. 2), not including those installed around the injection well constructed by the U.S. Geological Survey, described hereafter. Geologic data were obtained from three core holes (fig. 2), all installed by rotary wireline coring techniques. Rotary core hole 1 was cored from land surface to a depth of $43 \mathrm{~m}$; however, core recovery in this hole was very poor. Rotary core hole 2 was cored from land surface to a depth of $50 \mathrm{~m}$, at that site, with almost complete core recovery except for intervals from 9 to $13 \mathrm{~m}$ and from 25 to $27.5 \mathrm{~m}$. The third core hole was cored in the interval from $30 \mathrm{~m}$ to $43 \mathrm{~m}$, which includes the saturated portion of the Ogallala Formation at that location. Core recovery was almost complete.

Additional geologic information was obtained from a trench excavated in the lake bank, and a pit (fig. 2) in the lake floor. The trench was dug with a backhoe to a depth of $3.3 \mathrm{~m}$. The trench was started at the lake floor, which consists of hard siliceous caliche that cannot be excavated with a backhoe, and was continued to the upper edge of the playa excavation, which is $7 \mathrm{~m}$ higher in altitude than the playa floor. By digging the trench into the sloping bank, it was possible to sample the top $7 \mathrm{~m}$ of material in a fresh open cut. The pit was excavated by blasting an opening in the siliceous layer with dynamite, and then excavating the loose rock and underlying unconsolidated material to a depth of $3.3 \mathrm{~m}$ with a backhoe. 
Initial plans were to use one or more of the dual-purpose wells for injection experiments; however, long-term pump tests on we11 1 and shortterm tests on wells 2 through 4 indicated that all four wells were poorly developed and subject to hydraulic-head losses during withdrawal or injection. Attempts to further develop the wells were unsuccessful; consequent1y, a new injection well was drilled about $17 \mathrm{~m}$ from well 1 . This well was drilled to a diameter of $0.3 \mathrm{~m}$, using biodegradable drilling fluid. The well was completed with $0.25-\mathrm{m}$ diameter wire-wound screen from a depth of 32 to $44 \mathrm{~m}$, which spans the saturated thickness of the aquifer. Blank casing of $0.25-\mathrm{m}$ diameter was cemented in place from a depth of $32 \mathrm{~m}$ to land surface. The well was developed by bailing, jetting, and swabbing to create a natural gravel pack.

Groups of observation wells, piezometers, and water samplers were located at distances of 2,5 , and $30 \mathrm{~m}$ from the injection well. In each group, the observation well was bottomed in the Triassic red beds at depths ranging from 44.2 to $44.8 \mathrm{~m}$. Each observation well was screened through the entire saturated thickness of the aquifer to obtain integrated water samples for the full depth. Four piezometers, consisting of 0.6-m long, 0.032-m diameter well points connected to land surface through $0.025-\mathrm{m}$ diameter pipe, were installed in a second hole at each location to measure water levels at various depths. A third hole in each group was equipped with four watersampling devices to obtain water samples at various depths within the aquifer. These samplers consist of polyethylene tubing fitted with a check valve, and are referred to as point samplers in this report. They are similar to the porous cup sampler described by Wood (1973), except the ceramic cup was replaced with a $0.50-\mathrm{m}$ long, $50-\mathrm{mm}$ diameter section of plastic well screen, and the tubing was packed in the well screen with clean sand or glass beads (fig. 3). In addition to the three sampling distances mentioned above, samples were obtained from a well screened from a depth of 41.5 to $42.7 \mathrm{~m}$, located $10 \mathrm{~m}$ (we11 A8, fig. 2) from the injection we11, and from a well (well All, fig. 2) screened from a depth of 30.5 to $41.8 \mathrm{~m}$, located $15 \mathrm{~m}$ from the injection well.

Depths and locations relative to the injection well of the various observation we11s, piezometers, and samplers used in the injection-well experiments are shown in figure 4. The injection we11 and the observation we11s I-1, I-4, and I-7 are bottomed in the Triassic red beds at depths ranging from 44.2 to $44.8 \mathrm{~m}$. The preinjection and prepumping depth to water was about $32 \mathrm{~m}$, and the wells are screened through the saturated part of the aquifer. Screened intervals also are shown in a cross-sectional view in figure 5 , so the relative vertical positions of the screens can be compared. Altitudes of the screen positions are listed in table 2. A single-point electrical resistivity $10 \mathrm{~g}$ made in well $\mathrm{I}-1$, located $2 \mathrm{~m}$ from the injection we11, also is shown in figure 5. In general, deflections of the log trace to the left indicate greater clay content of the sediments at that level and, by inference, less hydraulic conductivity. 


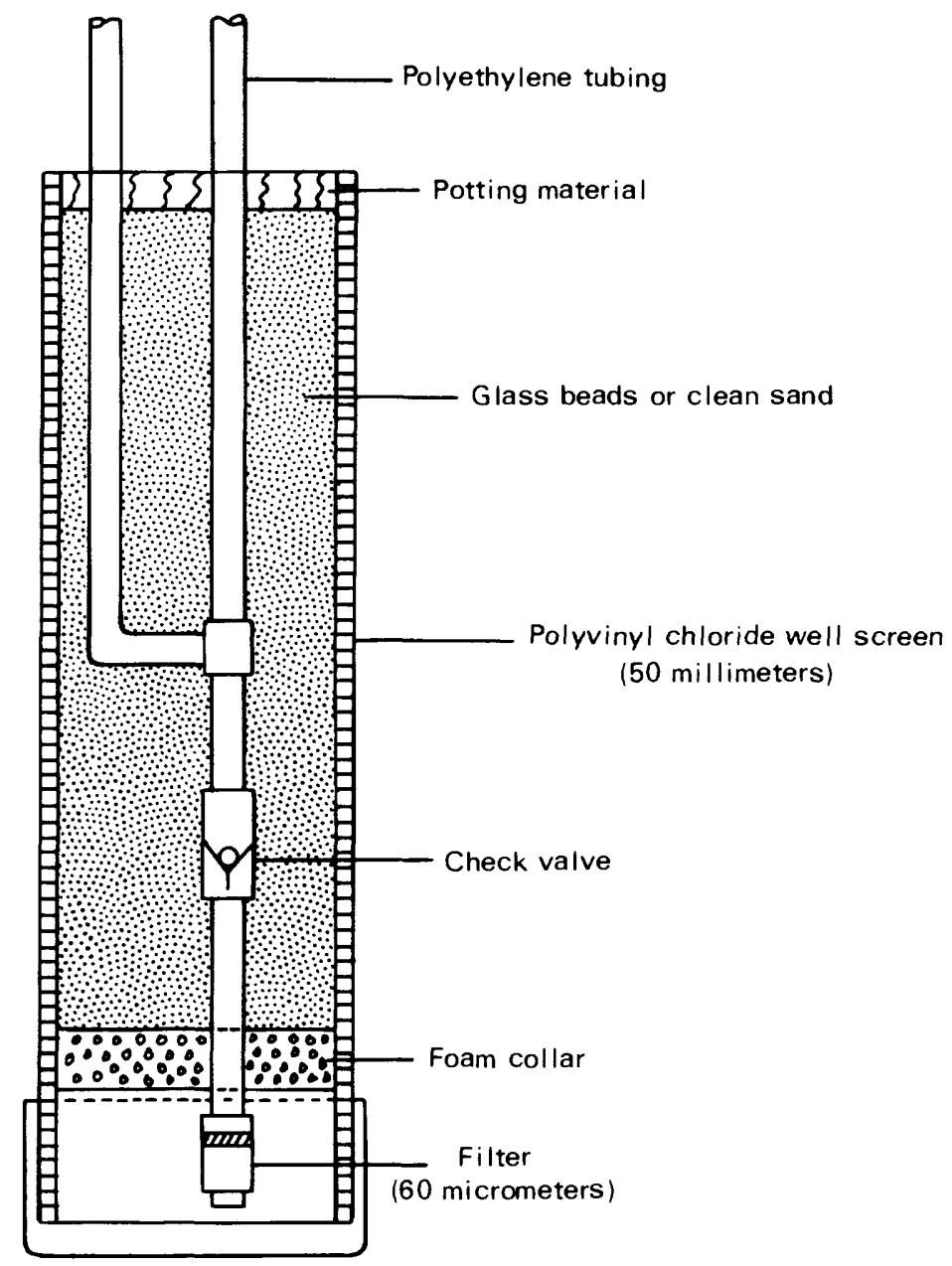

Figure 3.--Construction of the point samplers used in this investigation. 


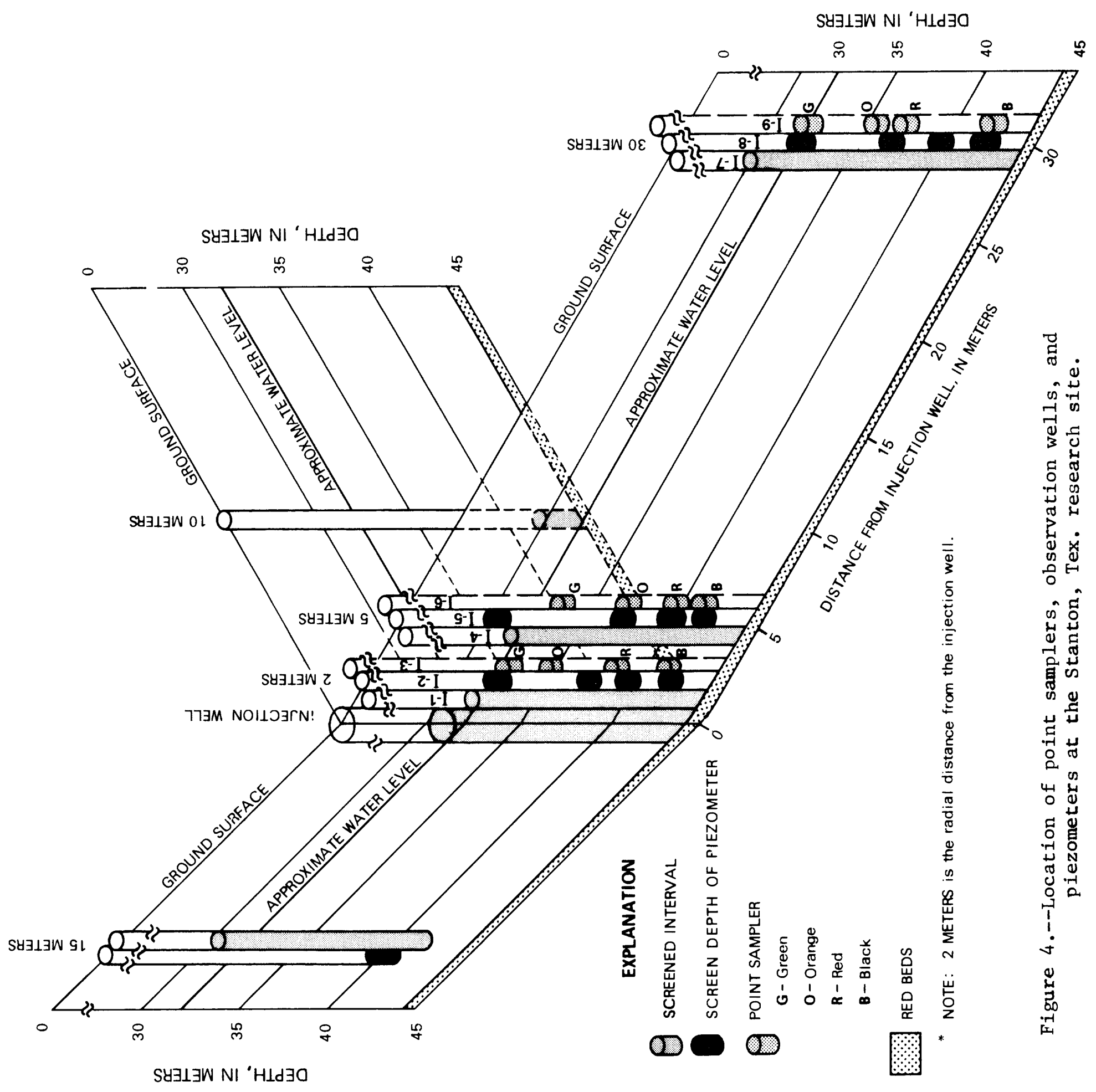



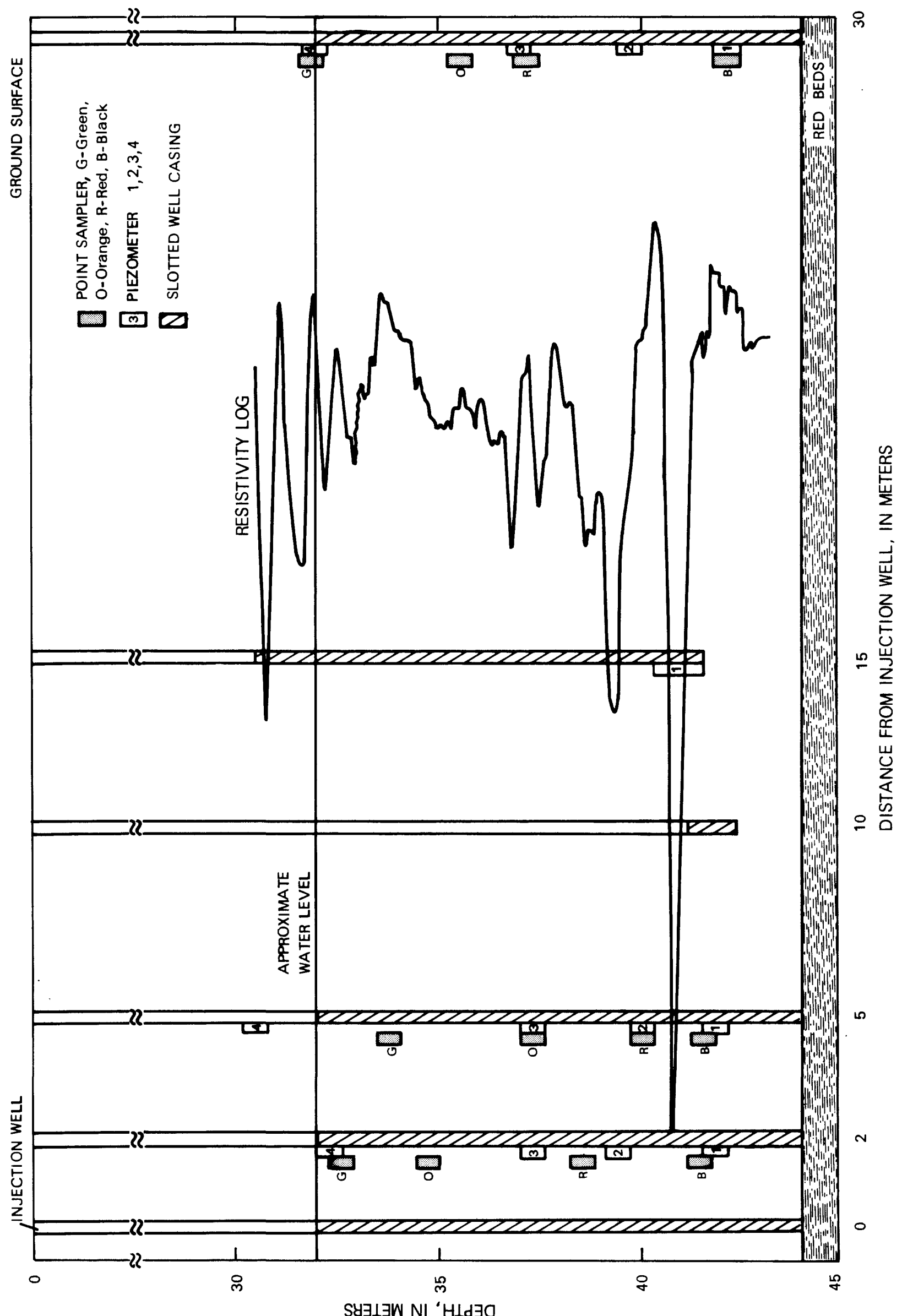

 
Table 2.--Depth and type of sampling Zocations

$$
\text { [p.s. = point sampler] }
$$

\begin{tabular}{|c|c|c|c|}
\hline $\begin{array}{l}\text { Radial distance } \\
\text { from injection } \\
\text { well } \\
\text { (m). }\end{array}$ & $\begin{array}{l}\text { Type of } \\
\text { sampler }\end{array}$ & $\begin{array}{l}\text { Range of } \\
\text { vertical } \\
\text { sampling } \\
\quad(\mathrm{m})\end{array}$ & Comments \\
\hline 2 & We11 & $32.0-44.2$ & Integrated sample. \\
\hline 2 & B1ack p.s. & $41 \cdot 3-41.9$ & \\
\hline 2 & Red p.s. & $38.4-39.0$ & \\
\hline 2 & Orange p.s. & $34.5-35.1$ & \\
\hline 2 & Green p.s. & $32.3-32.9$ & \\
\hline 5 & We11 & $32.0-44.2$ & Integrated sample. \\
\hline 5 & Black p.s. & $41.5-42.1$ & \\
\hline 5 & Red p.s. & $40.0-40.6$ & $\begin{array}{l}\text { Inoperable; an adjacent pie- } \\
\text { zometer was used. }\end{array}$ \\
\hline 5 & Piezometer & $39.9-40.5$ & Screened piezometer tube. \\
\hline 5 & Orange p.s. & $37.2-37.8$ & \\
\hline 5 & Green p.s. & $33.5-34.1$ & Above the water table. \\
\hline 10 & We11 & $41.5-42.7$ & Sampled only a small zone. \\
\hline 15 & We11 & $30.5-41.8$ & Integrated sample. \\
\hline 30 & We11 & $32.0-44.2$ & Integrated sample. \\
\hline 30 & Black p.s. & $42.1-42.7$ & \\
\hline 30 & Red p.s. & $37.1-37.7$ & \\
\hline 30 & Orange p.s. & $35.4-36.0$ & \\
\hline 30 & Green p.s. & $31.7-32.3$ & Above the water table. \\
\hline
\end{tabular}


Water samples from the fully penetrating observation we1ls at 2 , 5 , and $30 \mathrm{~m}$ were obtained using small-diameter gas-operated sample pumps (described by Signor, 1978). Intakes for these pumps were manifolded to draw water into the pump from points spaced $1.6 \mathrm{~m}$ apart through the full initially saturated thickness of the aquifer. Pumps were in continuous operation, and samples for chemical analysis were collected periodically from the pump outflow.

The point samplers were operated in the following manner: (1) Pressure was maintained in the lines by a $6,895-\mathrm{Pa}$ check valve, which prevented water from entering the sampler before the start of the sampling sequence; and (2) water was drawn into the sampler by releasing pressure and applying a vacuum for 2 min at selected sampling times. Once water had been drawn through the sampler into the lines, the vacuum was removed and pressure was applied from a nitrogen-cylinder tank to bring the sample to land surface. A11 the samplers performed as intended except the inoperative red-coded point sampler at $5 \mathrm{~m}$ from the injection well. However, the green point sampler at $30 \mathrm{~m}$ was above or so near the water table that samples could not be obtained during any of the three tests. Both the 2-m and 5-m green samplers were sampled during the March test, but the water table had declined to the point that only the 2-m green sampler could be sampled during May. None of the green point samplers could be sampled during the August test because of additional declines in the water table between May and August 1978 .

During the March 1978 test, the wells at 10 and $15 \mathrm{~m}$ were pumped using sma11-diameter bladder-type pumps designed by R. F. Middelburg of the U.S. Geological Survey. During the May 1978 and August 1978 tests, these wells, and the piezometer completed at the same distance and depth as the 5-m red sampler, were sampled using pumps that consisted of check-valve-equipped copper cylinders connected to the surface by two lengths of polyethylene tubing. Water enters these samplers because of hydrostatic pressure on the submerged check valve, and is expelled at surface by application of pressure from an air compressor. These samplers were operated by alternately applying and releasing pressure for 1-min intervals through a timer-equipped solenoid valve. In addition, point samples also were collected at several depths and times during the August 1978 test from the 15-m we11, using a straddle-packer arrangement on one of the copper-tube pumps; these samples are footnoted in the appropriate tables.

Water levels were monitored in piezometers consisting of 25-mm diameter, 0.6-m long we11 screens emplaced in we11s I-2, I-6, and I-8 at depths shown in the cross section (fig. 5). Water levels also were measured in observation wells A3, A6, A9, A10, and A14 (fig. 2). Pumping or injection rates into the production well were measured using inline propellor-driven Sparling* flowmeters. Periodic flowmeter traverses were made in the injection well

*The use of trade names in this report is for identification purposes on1y and does not imply endorsement by the U.S. Geologica1 Survey. 
during injection tests to determine which zones being recharged. Periodic neutron soil-moisture logs were made in selected observation wells to monitor changes in water stored in the capillary fringe and the unsaturated zone caused by changes in the altitude of the water table.

With the exception of the yeast and fluorocarbons, all tracers were added to the injection stream with chemical-feed pumps during each of the experiments; fluorocarbon tracers were contained in pressurized cylinders. Their flow into the injection water was controlled by a needle valve in the connecting tubing.

During the August 14, 1978, pulse-injection test, the injection stream was diverted through a sample chamber containing the tracers, as shown in figure 6. The sample chamber operated as follows: Prior and subsequent to injection of the tracer-bearing pulse, valve 1 was opened, and valves 2 and 3 were closed, so flow bypassed the chamber. Immediately prior to the pulse-injection test, the chamber was filled with the tracer solution. To start the pulse, valve 1 was closed and valves 2 and 3 were opened simultaneously to divert the injection stream through the chamber, and thus create a pulse of tracer-tagged water in the stream. After 2 min, valves 2 and 3 were closed, and valve 1 reopened to avoid gradual flushing of tracer-bearing water from the chamber.

\section{GEOLOGY}

The Stanton, Texas research site is located on the southern edge of the Ogallala aquifer, which is the principal water-bearing formation in the region. The aquifer is wholly contained within the Ogallala Formation of Miocene age. The Ogallala Formation is underlain by red Triassic shales and sandstones, which form an impermeable lower boundary to the aquifer at a depth of 44 to $45 \mathrm{~m}$ below land surface (fig. 7).

The lithologic column from land surface to the base of the aquifer, as determined from examination of the trench and pit and of cores from test holes (fig. 2) is shown in figure 7. The keyed numbers along the column refer to tables 3, 4, and 5, where additional information is given about the mineralogy, porosity, or hydraulic conductivity at that point in the column.

The sediments in this column are primarily alluvial material derived by erosion of the southern extensions of the Rocky Mountains in New Mexico. The sediments range from well-sorted subangular-to-angular argillaceous sands to poorly-sorted gravels, with occasional pebbles having diameters as much as $40+\mathrm{mm}$. Zones of calcium carbonate caliche occur in the upper part of the column, commonly associated with colloidal silica leached from the aggrading soil profile. One such zone occurs from about 6 to $7 \mathrm{~m}$; it is extremely hard and difficult to penetrate during drilling. Several relatively impermeable clay lenses are present in the section, but are not continuous enough to be stratigraphic markers. The sediments are grossly inhomogenous, and hydraulic conductivities vary widely through the section. 


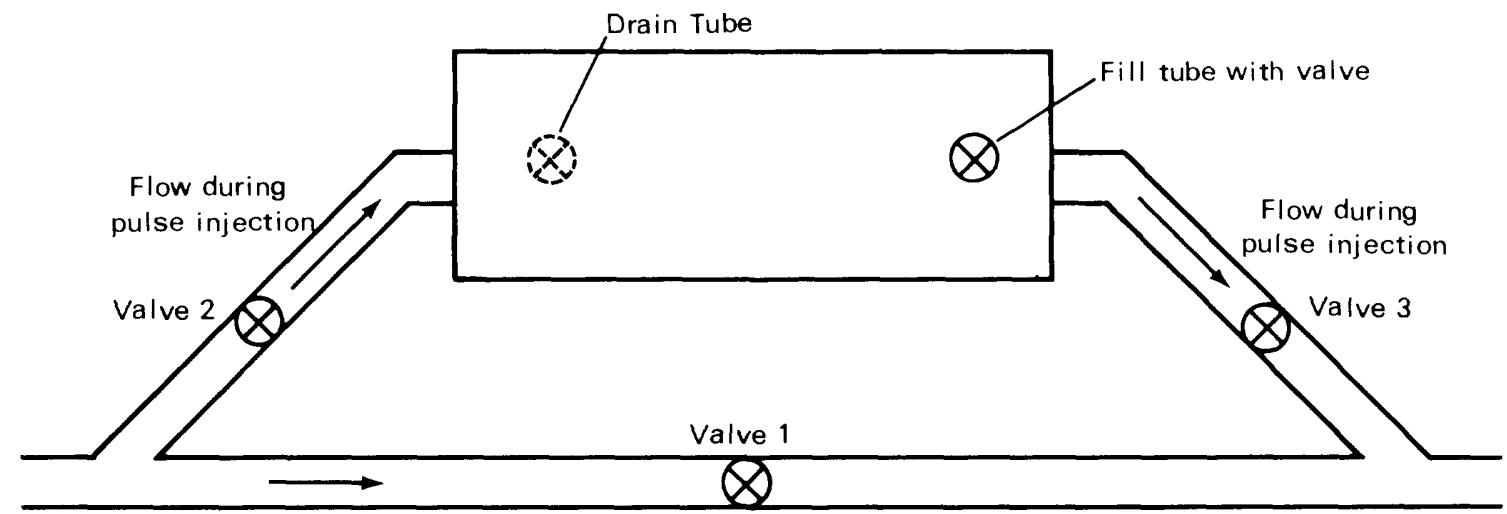

Flow during test

Figure 6.--Bypass chamber used to produce a virtually instantaneous pulse of tracer-tagged water in the injection stream.

(Valve 1 is open during bypass flow, and valves 2 and 3 are open during pulse injection.) 


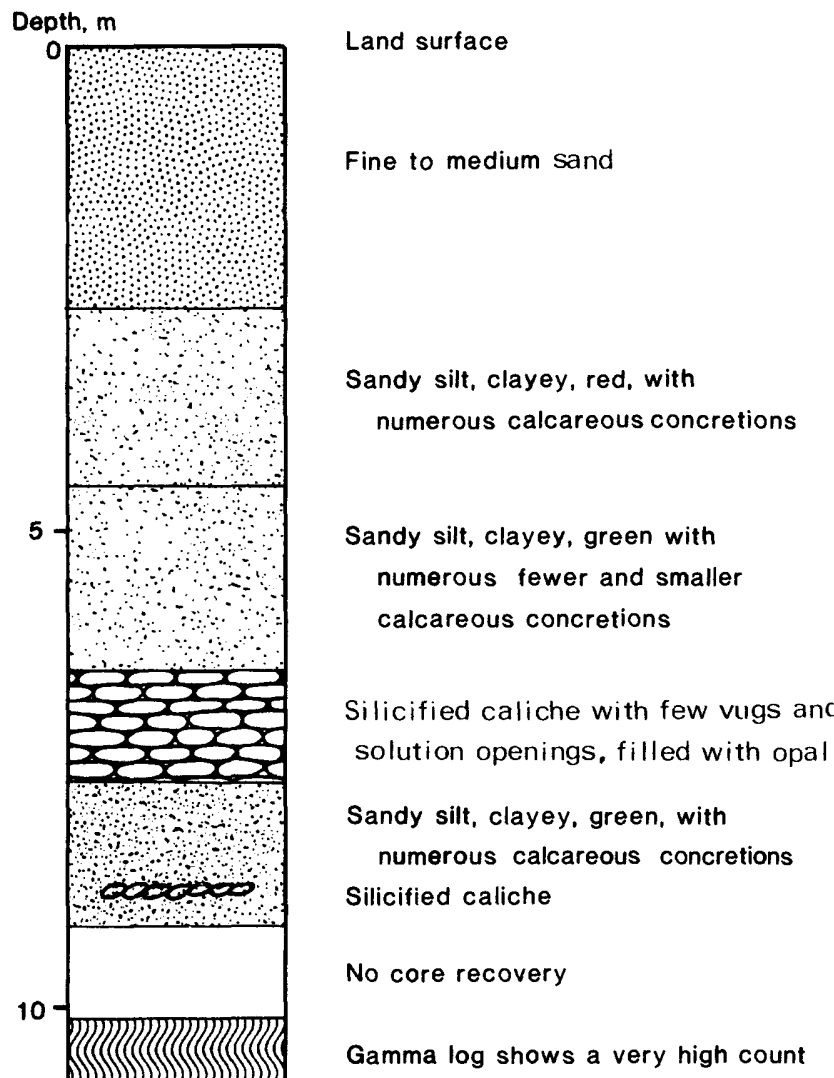

No core recovery

Gamma log shows a very high count

Conglomerate, silty clay, grey, calcareous deposits

Fine clayey sand, brownish red

Conglomerate, clayey sand, small cemented sandstone nodules

Silty sand, with small caliche nodules, reddish brown

Coarse sand

Silty fine sand, with tan caliche nodules

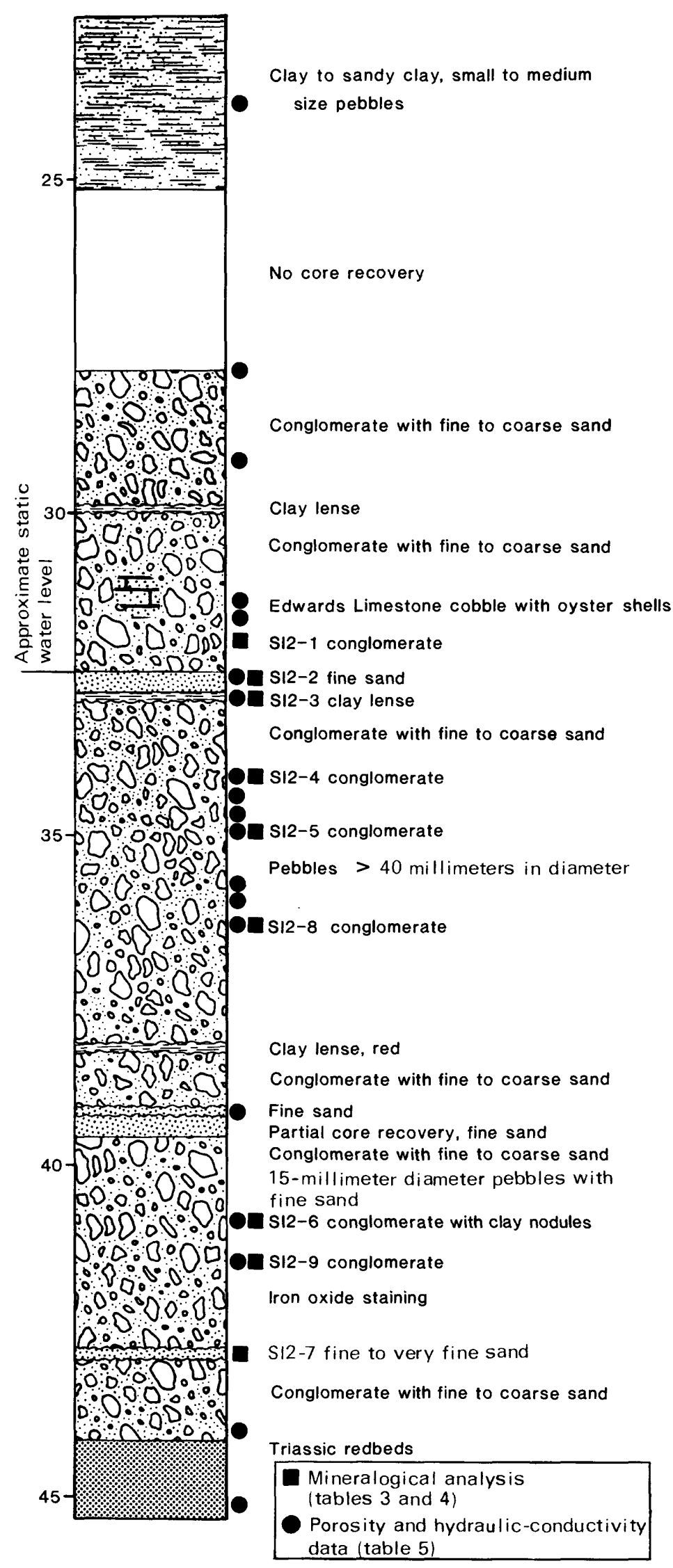

Figure 7.--Lithologic column from the Stanton, Tex. site with point locations of mineralogical analyses, porosity, and hydraulic-conductivity determinations. 


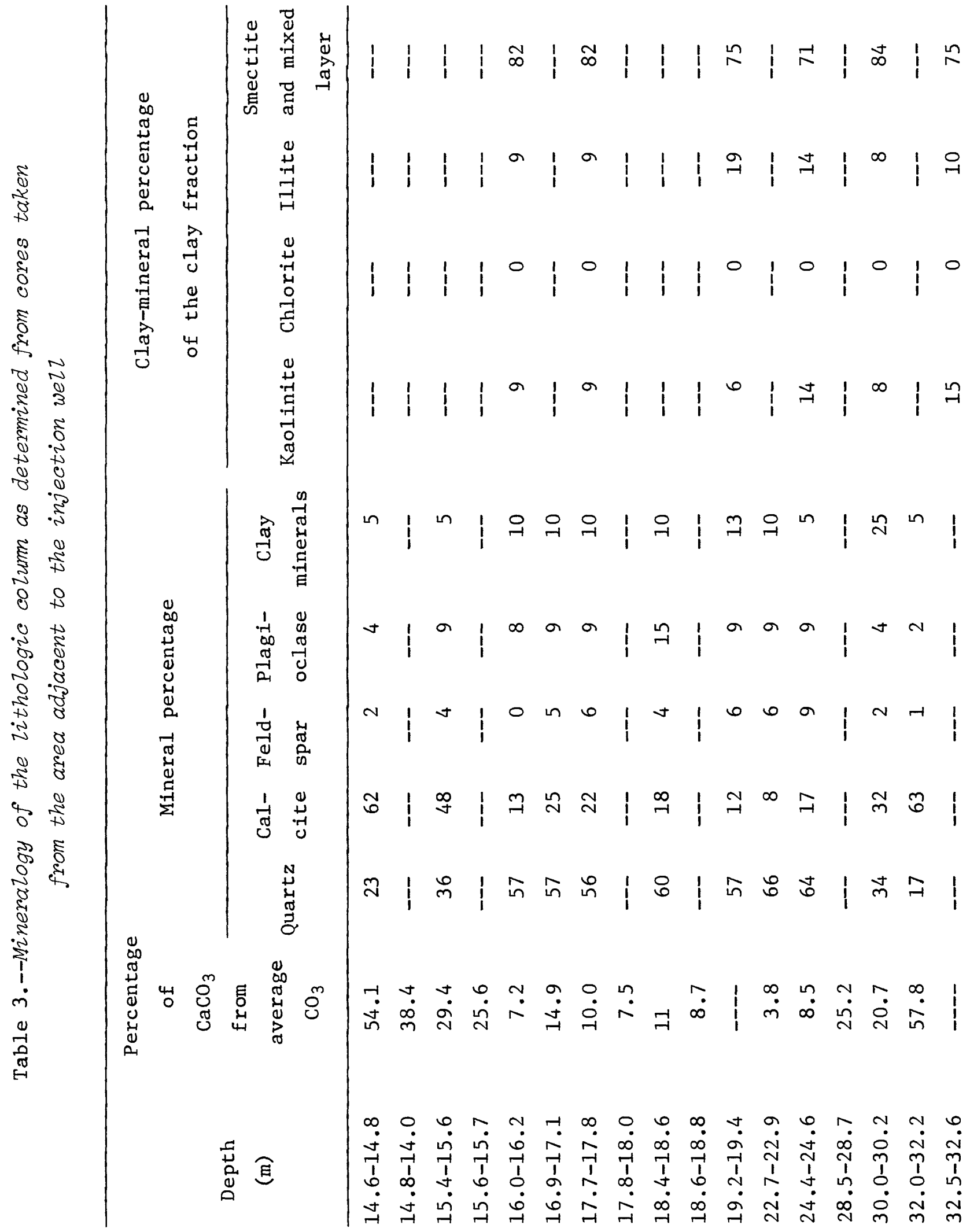




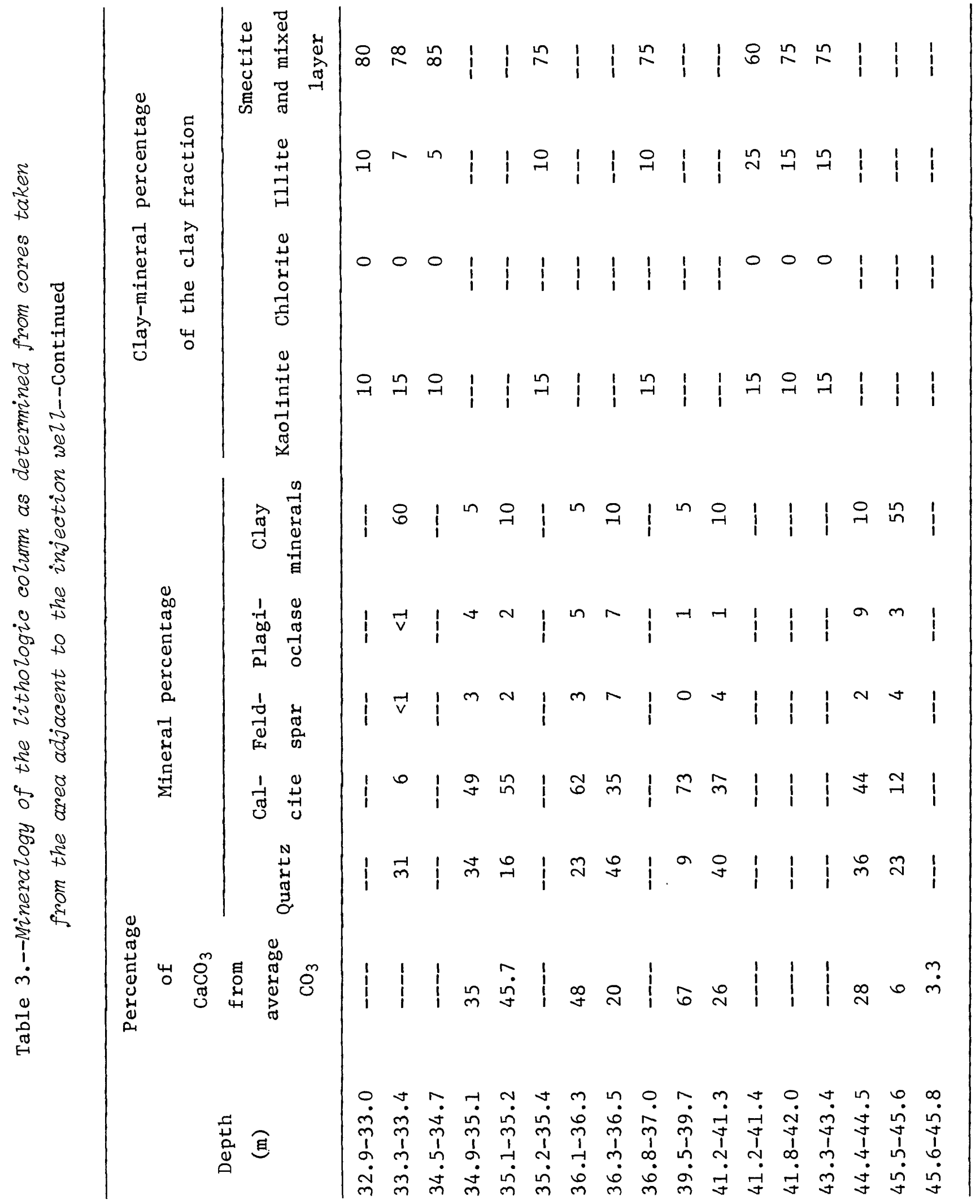




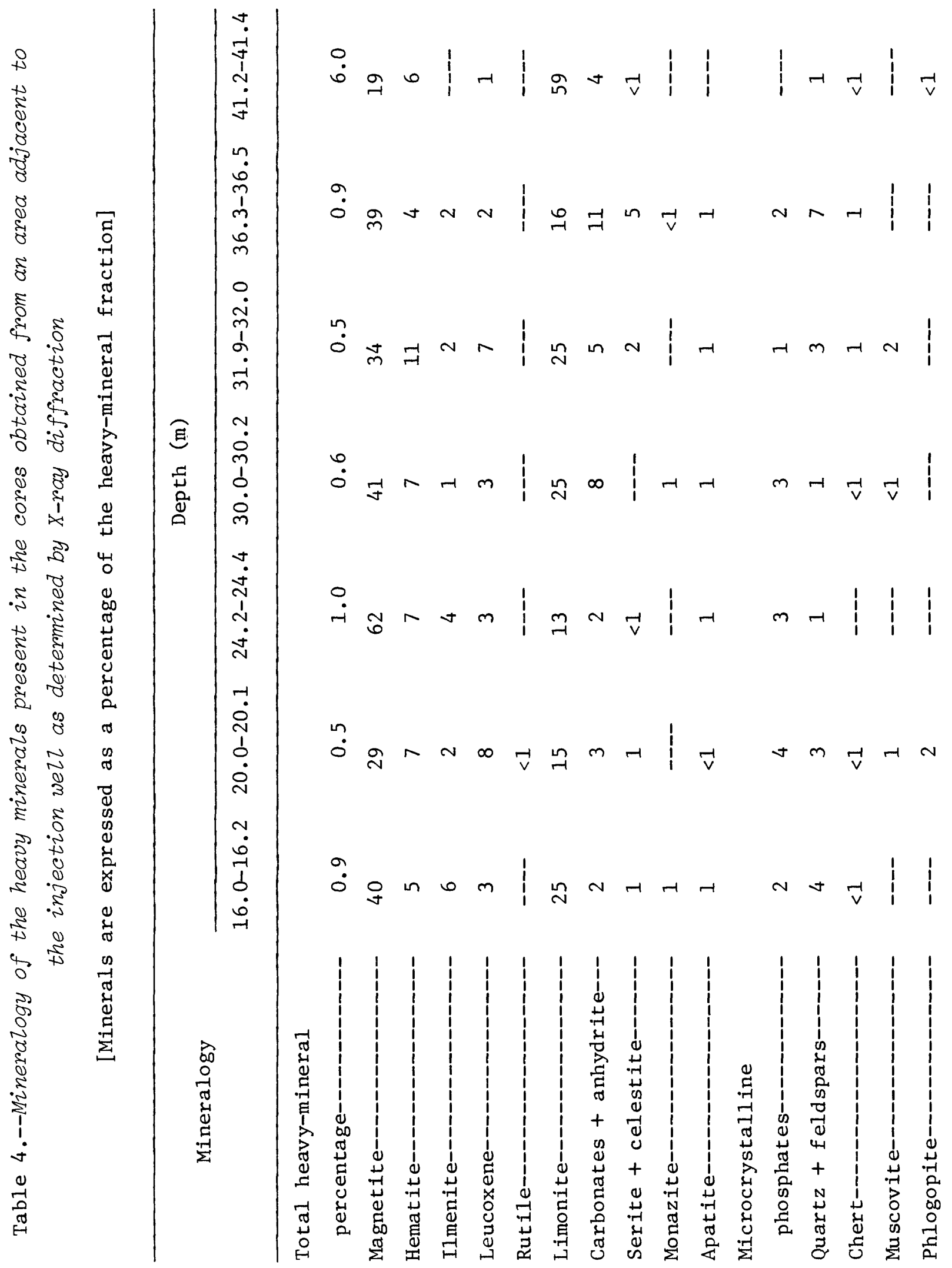




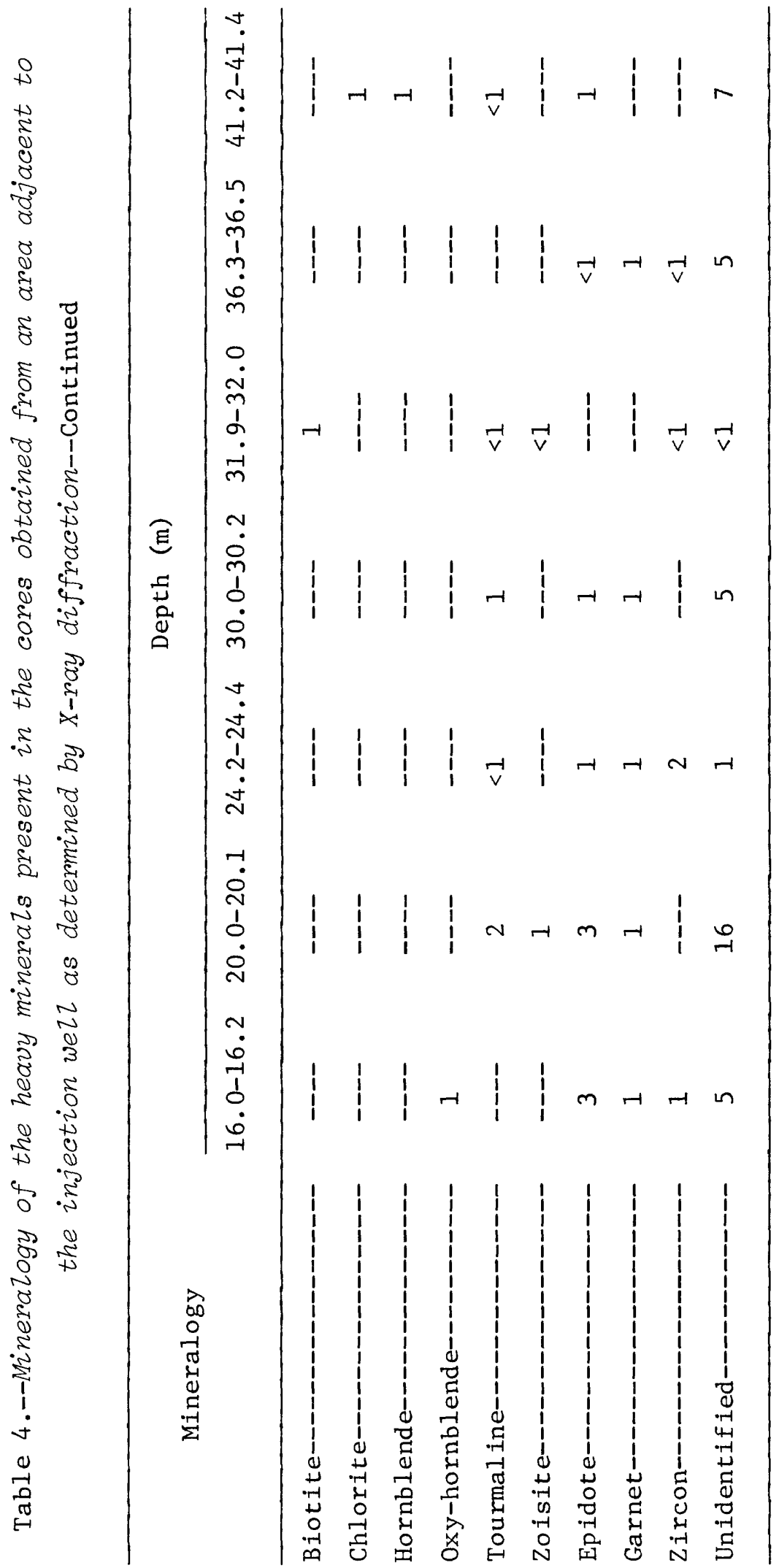


Table 5.--Porosity and hydraulic-conductivity data determined from core samples from an area adjacent to the injection well

\begin{tabular}{lll}
\hline $\begin{array}{c}\text { Sample } \\
\text { number }\end{array}$ & $\begin{array}{c}\text { Porosity } \\
\text { (percent) }\end{array}$ & $\begin{array}{c}\text { Hydraulic } \\
\text { conductivity } \\
(\mathrm{m} / \mathrm{d})\end{array}$ \\
\hline SI2-1 & 26.5 & $8.2 \times 10^{-2}$ \\
SI2-2 & 27.8 & $2.1 \times 10^{-1}$ \\
SI2-3 & $--1-$ & $3.1 \times 10^{-5}$ \\
SI2-4 & 24.0 & $2.4 \times 10^{-2}$ \\
SI2-5 & 28.7 & $3.3 \times 10^{-1}$ \\
SI2-8 & 25.5 & $1.4 \times 10^{-1}$ \\
SI2-6 & 28.2 & $3.6 \times 10^{-1}$ \\
SI2-9 & 21.3 & $1.2 \times 10^{-2}$ \\
SI2-7 & 30.9 & $8.2 \times 10^{-2}$ \\
\hline
\end{tabular}




\section{HYDROLOGY}

The regional ground-water flow system in the Southern High Plains has been discussed by Cronin (1964), and will not be described in detail here. The Ogallala aquifer underlies the entire Southern High Plains. Ground water occurs under unconfined (water-table) conditions throughout most of the area. The hydraulic gradient averages between 1.3 and $3.0 \mathrm{~m} / \mathrm{km}$.

At the Stanton experimental site, the regional flow system is affected by recharge from the excavated playa lake, and by pumpage of ground water for irrigation and domestic use. The hydraulic gradient at the site slopes from the point of recharge below the lake toward the southeast, even when the lake is dry. Apparent1y, deep percolation in the unsaturated zone continues during those periods, maintaining recharge to the ground-water reservoir. A perched ground-water zone occurs at a depth of about $18 \mathrm{~m}$ throughout the site. Percolation from this perched zone may provide an additional source of recharge during periods when the lake is dry.

The average depth to the regional water table at the Stanton site was about $33.5 \mathrm{~m}$ below land surface during 1970-78. Measured water-level depths have ranged from about 32.0 to $36.0 \mathrm{~m}$, but there are no apparent long-term trends in water levels. Depth to water in the injection well and the saturated thickness of the aquifer immediately prior to the start of the various pumping and injection tests are tabulated below:

Date

Depth to water

(m)
Saturated thickness

(m)
1/June 1973
32.9
11.1
I/ Ju1y 1976
32.8
11.2
Mar. 1977
31.7
12.3
Mar. 1978
33.3
10.7
May 1978
33.2
10.8
Aug. 1978
35.2
8.8
Dec. 1978
34.3
9.7

$1 /$ Depth to water measured in we11 1 , located $17 \mathrm{~m}$ west of the injection we11. 
In addition to the seasonal variations, water levels fluctuate diurnally in response to changes in atmospheric pressure. These daily fluctuations average about $30 \mathrm{~mm}$, but can be as large as $300 \mathrm{~mm}$ when a major barometric-pressure change occurs.

A number of laboratory analyses of porosity and of hydraulic conductivity were made on cores obtained while drilling observation well I-2. The depths, porosity, and hydraulic conductivity values for these cores are listed in table 5. Porosity values are relatively uniform with depth, but the hydraulic-conductivity values show great variability. This variation is consistent with the variations in the injection loss with depth in the injection well, although the injection losses are affected by well-bore plugging as well as by variations in hydraulic conductivity of the aquifer materials.

\section{ANALYTICAL METHODS}

\section{Genera1}

Precautions were taken during this investigation to insure that the water samples represented the natural environment as accurately as possible. Some constituents present in water are unstable, and change before they can be analyzed in the laboratory. Measurement of these constituents was done either at the well site or the samples were preserved for future analysis according to accepted methods. Alkalinity and $\mathrm{pH}$ were measured immediately after sample collection, according to the methods of Wood (1973). The alkalinity titration was performed automatically by using a syringe pump that delivered the acid into the water sample; a strip-chart recorder was used to plot the titration curve. Bromide and iodide were monitored at the well site with Orion specific-ion electrodes and an Orion 701 digital meter. Specific conductance and water temperature were measured at the well site, and samples to be used for complete chemical analyses were filtered and acidified according to the methods of Wood (1973) and Brown and others (1970). Boron was analyzed in the laboratory according to the dianthrimide method using an ultraviolet visible spectrophotometer, and bromide was determined both by the catalyticoxidation technique and the specific-ion-electrode method (Brown and others, 1970; Orion Research, Inc., 1977). A11 other inorganic analyses were performed in a U.S. Geological Survey central laboratory according to standard methods (Brown and others, 1970).

The yeast analysis was performed at the well site using standard techniques. Known volumes obtained from serial dilutions of the samples were filtered through membrane filters, as described by the American Public Health Association and others (1971). Filters were placed on absorbent pads saturated with M-Green Yeast and Mold Broth (BBL no. 11286) and incubated at $30^{\circ} \mathrm{C}$ for 36 hours. Colonies were counted under low magnification. 
Fluorocarbon-Compound Analysis

A11 the fluorocarbon measurements were made at the test site to prevent tracer loss by volatilization. Two methods of analysis were used: A method for concentrations in the nanogram per liter (part per trillion) range, and a method for concentration in the milligram per liter (part per million) range.

The instrumentation for determining fluorocarbon concentrations in the nanogram per liter range is shown schematically in figure 8; it consists basically of two parts: (1) A sample-handling system that removes the tracer from a 5- to $25-\mathrm{mL}$ water sample by gas stripping and concentrates it in a trapping loop for injection into a chromatographic system; and (2) a Varian 3700 series gas chromatograph equipped with an electron-capture detector for measurement of the gas sample stripped out of the water. The description of the analytical procedure is given by Thompson (1976) and Thompson and Hayes (1979).

During the final August experiment, tracer analyses were performed by a more conventional chromatographic technique. During the August test, tracers were measured by direct injection of $1-\mu \mathrm{L}$ water samples onto a single column attached directly to the detector; thus, the tracers were chromatographically separated from each other and from the water. The limit of detection for most tracers by this method is in the microgram per liter range. This method was used because extremely high sensitivity was not needed, and it is more rapid than the sample-stripping technique. The chromatographic column packing used for the direct-injection procedure was 100-120 mesh proapack Q (Alltech, Inc., Arlington Heights, I11.), and the column dimensions were $1 \mathrm{~mm}$ ID $\mathrm{x} 1 \mathrm{~m}$ long.

During the injection of the fluorocarbons, a problem developed with time that caused large variations in the input concentration. The reasons for this fluctuation are suspected to be pressure changes in the injection vessels and the main flow 1ine, and interaction of the tracers with organic matter inside the injection plumbing. Fluorocarbon concentrations in the flow stream remained anomalously large even after addition of the fluorocarbons had ceased. The variation is shown in figures 9 through 11 . Because of the large fluctuations in input concentrations, initial injection concentrations $\left(C_{0}\right)$ during the August 1978 tests were computed as average concentration throughout their respective injection periods.

\section{Nonvolatile Organic-Compound Analysis}

Dissolved organic carbon (DOC) was determined within $12 \mathrm{~h}$ of sample collection during each test. DOC was determined at the well site using a Beckman 915 Carbon Analyzer, which has a DOC detection limit of $3 \mathrm{mg} / \mathrm{L}$ and a precision of $0.25 \mathrm{mg} / \mathrm{L}$, during the March and August tests. During the May test, on-site DOC determinations were made using a Technicon DOC Analyzer, 


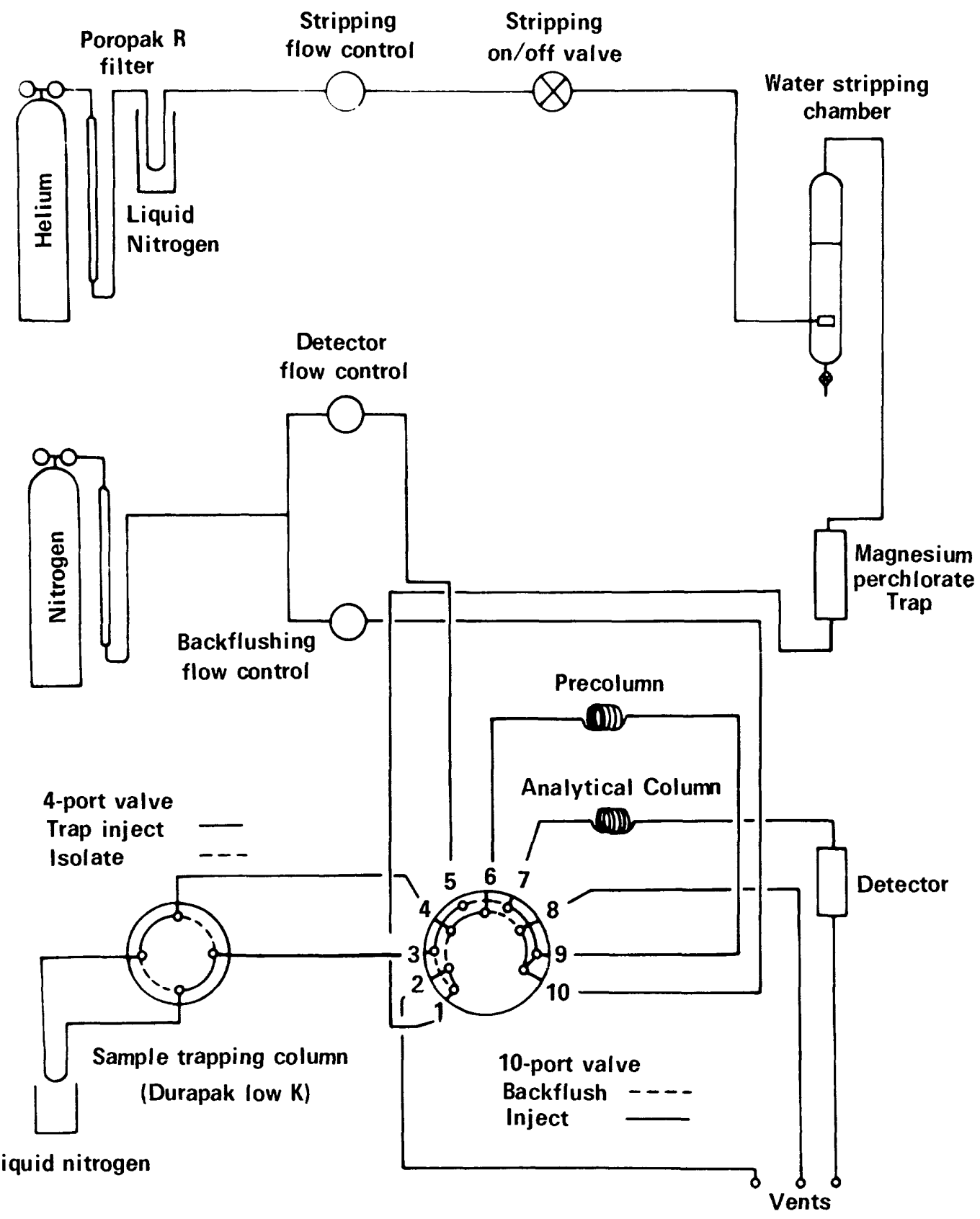

Figure 8.--Stripping, concentration, and analytical system for fluorocarbons in the nanogram per liter range.

(After Thompson, 1976.) 


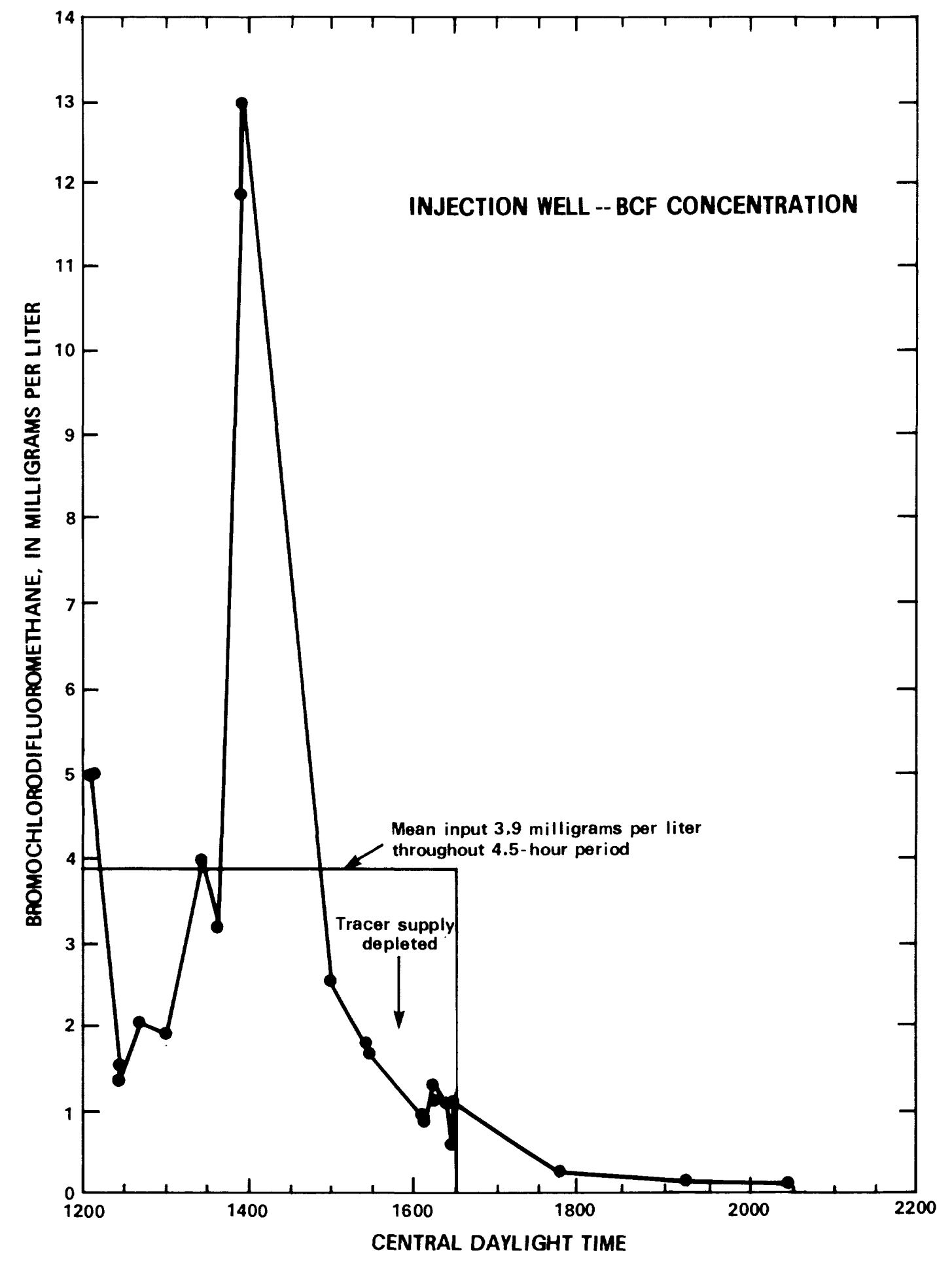

Figure 9.--Variation of input concentration with time of the fluorocarbon bromochlorodifluoromethane during the first tracer experiment, August 8, 1978. 


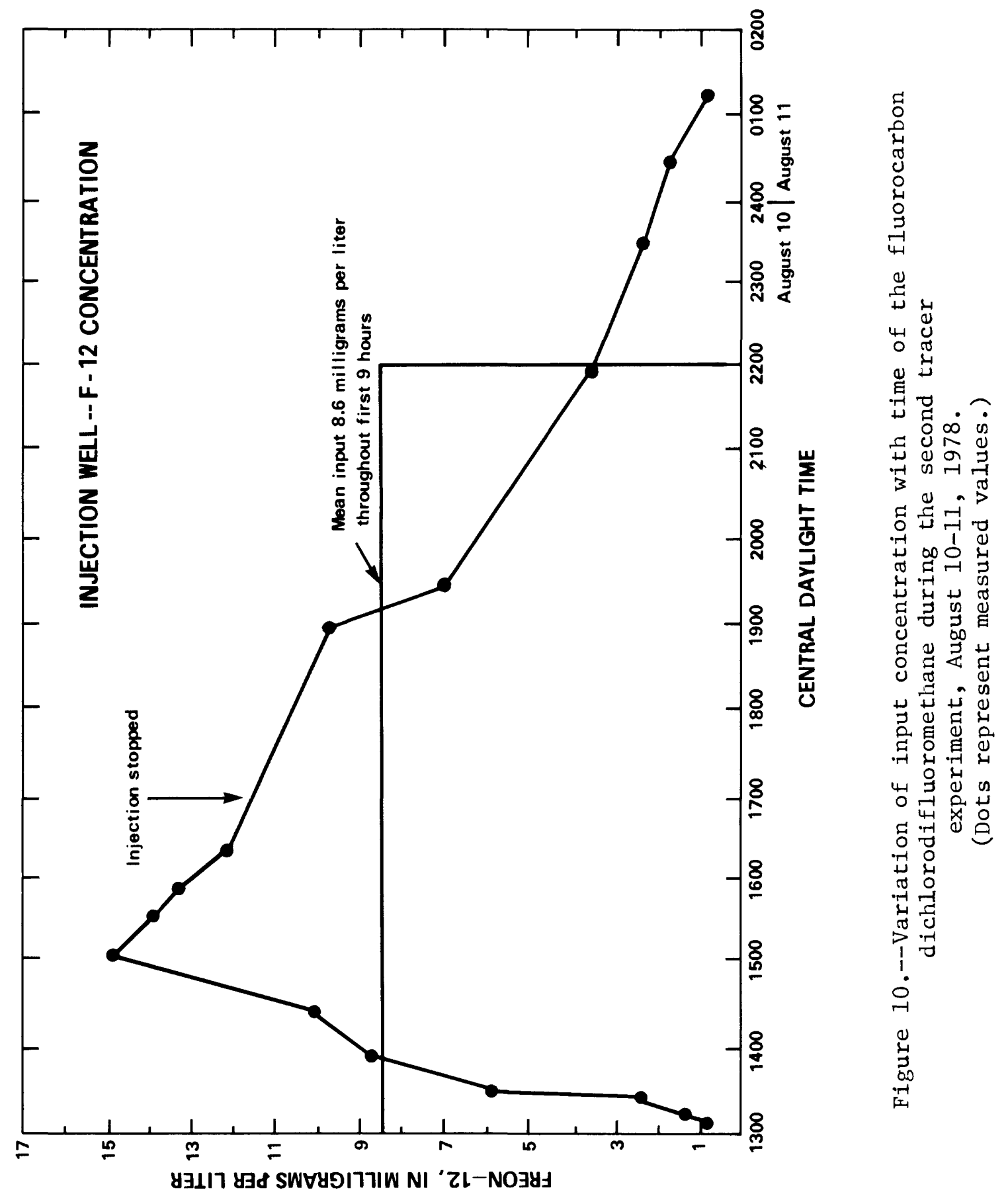




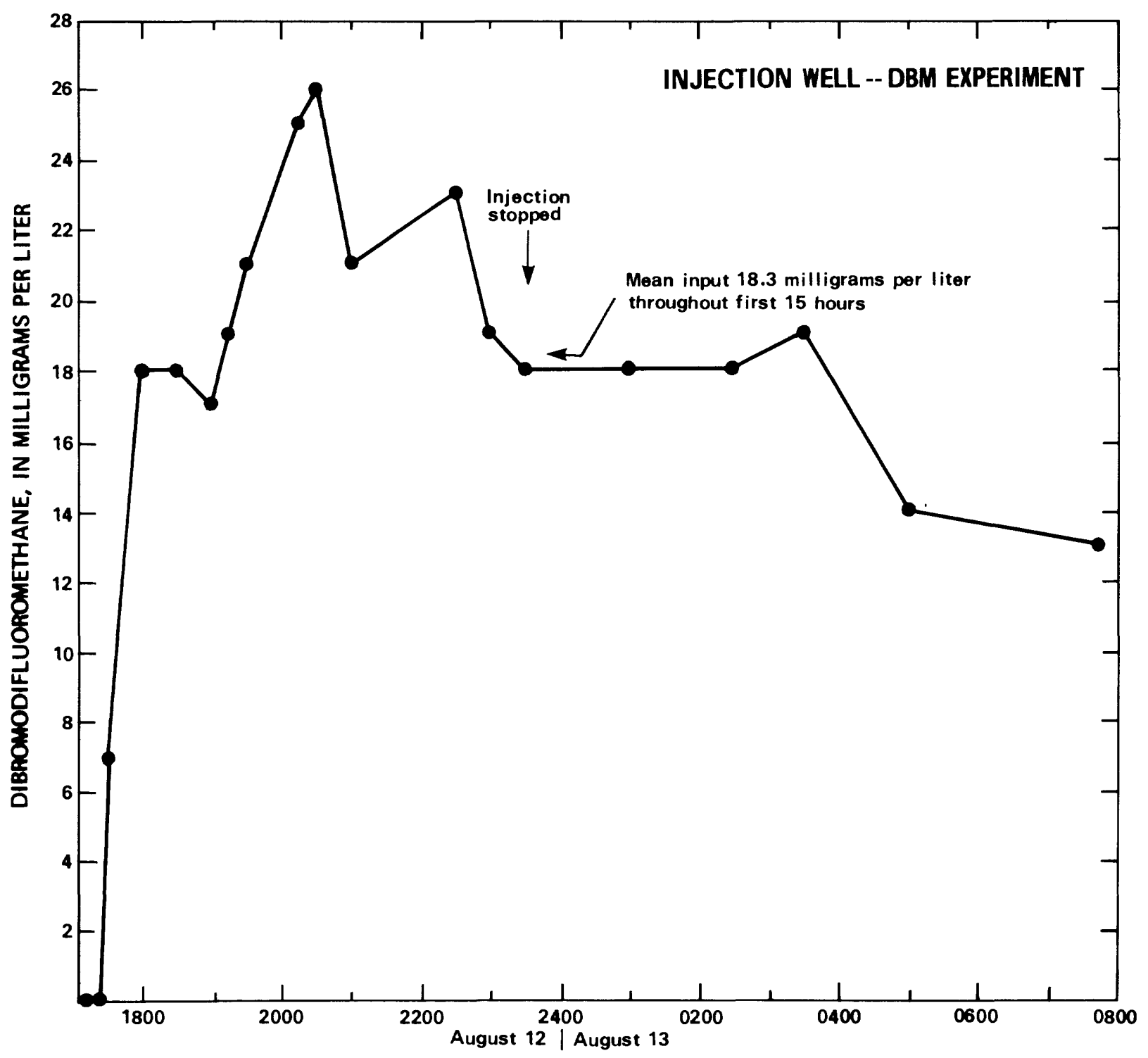

CENTRAL DAYLIGHT TIME

Figure 11.--Variation of input concentration with time of the fluorocarbon dibromodifluoromethane during the third tracer experiment, August 12-13, 1978.

(Dots represent measured values.) 
which has a detection 1 imit of $0.1 \mathrm{mg} / \mathrm{L}$ and an accuracy of $\pm 0.02 \mathrm{mg} / \mathrm{L}$. The on-site DOC measurements for the May test were verified in the laboratory using the Beckman 915 Carbon Analyzer.

Phenolphthalein was analyzed at the well site at a $\mathrm{pH}$ of 12 and a wavelength of $560 \mathrm{~nm}$ using a Beckman Model DB spectrophotometer. Benzoate and aniline concentrations were measured in the laboratory by direct aqueous injection of 200- $\mu \mathrm{L}$ samples into a Varian 8500 liquid chromatograph, fitted with a $\mu$ Bondapack C18 column. Both compounds were detected at $221 \mathrm{~nm}$ on a Varichrome spectrophotometer, with sodium phosphate buffered to $\mathrm{pH} 7$ as the mobile phase. Ethylamine was determined by direct-injection gas chromatography. Ethanol during the March test was calculated as the difference between DOC and benzoate. Ethanol and ethanol plus acetate, neither of which is tabulated here, were determined as the difference between DOC and the remaining organic compounds during the August test.

\section{MARCH 1977 PUMPING TEST}

Three pumping tests were conducted in the vicinity of the injection we11, including a 48-h test using well 1 during June 1973, a 66-h test using we11 1 during July 1976, and a 48-h test using the injection well during March 1977. Additional tests were conducted using the injection well during development in April 1978. The analyses described hereafter are preliminary; the measured water-level data are not listed. However, they may be obtained from E. P. Weeks upon request.

Results of the two tests for we11 1 are not included. For the first test, the pumping rate was only $1.7 \mathrm{~L} / \mathrm{s}$, and drawdowns in nearby observation wells were so small that they were difficult to analyze. During the July 1976 test, the well was pumped at a rate of $4.0 \mathrm{~L} / \mathrm{s}$, the maximum rate that could be sustained without drawing the water level in the well below the pump bowls. Data collected during the tests from nearby observation wells gave information similar to that listed for the March 1977 (table 6) and March 1978 (table 9) tests. Water-level data from well 1 indicate that the wel1 was poorly developed and hydraulic-head losses across the gravel pack and screen were very large. After data from the July 1976 test were analyzed, it was decided to construct a new injection well. Anticipated yield of the new we11, based on the July 1976 test data, was 12 to $16 \mathrm{~L} / \mathrm{s}$.

A 48-h test was conducted using the new injection well during March 1977, to confirm the anticipated production rate and to stress the aquifer sufficiently to obtain large drawdowns, hence, obtaining more reliable test data than had been possible by pumping well 1 . Unfortunately, the well failed to produce at the anticipated rate. Initial production was only $4.1 \mathrm{~L} / \mathrm{s}$, which could not be sustained, and had to be reduced to $4.0 \mathrm{~L} / \mathrm{s}$ during the last few hours of the test.

During the test, water levels were measured in the various piezometers, in the fully penetrating observation wells located 2,5 , and $30 \mathrm{~m}$ from the 
injection well (figs. 2 and 4 ), in the partly penetrating observation wells located 10 and $15 \mathrm{~m}$ from the production we11, and in observation wells A3, $\mathrm{A} 6, \mathrm{~A} 8, \mathrm{~A} 9, \mathrm{~A} 10$, and A14 (fig. 2). Piezometers 1 and 4 in the 2-m nest were plugged, and repeated attempts to develop them were unsuccessful. Piezometer 4 at $5 \mathrm{~m}$ was above the water table, and piezometer 1 at $30 \mathrm{~m}$ was so near the water table that the water table declined below the piezometer early during the test. Reliable water-level data were obtained from the remaining piezometers and observation wells, although the water levels were significantly affected by barometric effects. Measured drawdowns were corrected for those effects using the method described by Weeks (1979), based on barometric records obtained at the site and a value for the pneumatic diffusivity of the unsaturated zone of $2,400 \mathrm{~m}^{2} / \mathrm{d}$.

Results of analysis of the corrected water levels by use of the Cooper and Jacob (1946) modification of the Theis (1935) nonequilibrium equation to determine values for transmissivity and the storage coefficient of the aquifer are listed for selected observation wells in table 6 . Transmissivity values determined from the various wells are reasonably consistent, and indicate that the transmissivity of the aquifer at the injectionwell site is about $220 \mathrm{~m}^{2} / \mathrm{d}$. Values for the storage coefficient, on the other hand, vary by two orders of magnitude. At least part of the variation arises from the fact that water enters the production well only in certain zones, creating an effect similar to that of partial penetration. These effects cause wide variations in the computed storage coefficient, as described by Weeks (1969). However, even data from the fully penetrating observation wells show considerable scatter in computed storage coefficient, and the value is extremely small for a water-table aquifer. Consequently, the storage coefficient values are not reliable for predicting drawdown or hydraulic-head buildup during long-term pumping or injection. Because the injection well had a sustainable production rate of almost exactly the same magnitude as that for well 1 , and because of the care exercised in drilling and developing the injection well, it was hypothesized that the relatively small yield was due to dewatering of a thin and very permeable zone near the water table, rather than to inadequate well development.

\section{MARCH 1978 INJECTION TEST}

The injection test conducted on March 1-2, 1978 was primarily designed to test the efficiency of the point samplers and pumps, and to provide experience with the operation of the treatment plant for use with subsequent lake-water injection tests. It was suspected that the inorganic tracers used during this test might be somewhat non-conservative; however, their function was simply to tag the water for evaluation of flow past the detection points. By reserving the most conservative tracers for subsequent tests, it was possible to reduce interference between experiments and avoid increasing the background concentration. The tracers used, time periods of tracer injection, and initial concentrations in the injected water are listed in table 7 . The iodide tracer was added to the injection water continuously 
Table 6.--Transmissivity and storage values computed for the Ogallala aquifer based on data from the March 1977 pumping test

[Computations are based on a discharge rate of $4.10 \mathrm{~L} / \mathrm{s}$ ]

\begin{tabular}{cccc}
\hline Wel1 & $\begin{array}{c}\text { Distance from } \\
\text { production wel1 } \\
\text { number }\end{array}$ & $\begin{array}{c}\text { Transmissivity } \\
\left(\mathrm{m}^{2} / \mathrm{d}\right)\end{array}$ & $\begin{array}{c}\text { Storage } \\
\text { (dimensionless) }\end{array}$ \\
\hline I-1 & 2 & 240 & $2 \times 10^{-3}$ \\
I-2-3 & 2 & 220 & $2 \times 10^{-4}$ \\
I-2-2 & 2 & 220 & $8 \times 10^{-4}$ \\
I-4 & 5 & 230 & $8 \times 10^{-4}$ \\
I-6-1 & 5 & 250 & $4 \times 10^{-3}$ \\
I-6-2 & 5 & 220 & $9 \times 10^{-4}$ \\
I-6-3 & 5 & 200 & $1 \times 10^{-4}$ \\
A-8 & 10 & 240 & $3 \times 10^{-3}$ \\
I-8-4 & 30 & 410 & $6 \times 10^{-4}$ \\
I-8-3 & 30 & 410 & $6 \times 10^{-4}$ \\
I-8-2 & 30 & 190 & $2 \times 10^{-2}$ \\
\hline
\end{tabular}


Table 7.--Tracer information for March 2978 injection test

\begin{tabular}{|c|c|c|c|c|}
\hline Tracer & $\begin{array}{l}\text { Length of } \\
\text { injection } \\
\text { (min) }\end{array}$ & $\begin{array}{l}\text { Start time } \\
\text { (CST) }\end{array}$ & Date & Initial concentration \\
\hline Iodide------ & continuous & 1200 & $3 / 1 / 78$ & $1.1 \mathrm{mg} / \mathrm{L}$ \\
\hline Ethano1----- & 54 & 1200 & $3 / 1 / 78$ & $50 \mathrm{mg} \mathrm{C} / \mathrm{L}$ \\
\hline $\mathrm{CBrCIF}_{2}----$ & 80 & 1200 & $3 / 1 / 78$ & $.7 \mu \mathrm{g} / \mathrm{L}$ \\
\hline----- & ---- & ---- & --- & $------\cdots-\cdot$ \\
\hline Ethanol----- & 54 & 2130 & $3 / 1 / 78$ & $45 \mathrm{mg} \mathrm{C} / \mathrm{L}$ \\
\hline Benzoate---- & 54 & 2130 & $3 / 1 / 78$ & $5 \mathrm{mg} \mathrm{C} / \mathrm{L}$ \\
\hline Freon-12---- & 60 & 2130 & $3 / 1 / 78$ & $2.5 \mu \mathrm{g} / \mathrm{L}$ \\
\hline
\end{tabular}


at a constant rate throughout the test. Consequent1y, its concentration increased each time the injection rate, described below, was reduced. The input concentration of $1.1 \mathrm{mg} / \mathrm{L}$ listed in table 7 is for an injection rate of $10 \mathrm{~L} / \mathrm{s}$. Concentrations for other injection rates, as 1isted in table 8, may be computed by the formula:

$$
C I=1.1 \mathrm{mg} / \mathrm{L} X(10 \mathrm{~L} / \mathrm{s} \div \mathrm{Qt})
$$

where

$$
\begin{aligned}
& \mathrm{CI}=\text { injection concentration, in milligrams per liter; and } \\
& \mathrm{Qt}=\text { injection rate at time } t \text {, in liters per second. }
\end{aligned}
$$

Other tracers were added during periods when the injection rate was constant, and are as listed in table 7.

For the test, ground water was pumped from well 3 into the horseshoe settling basin, where it was picked up by the 38-kW lift pump. Diatomaceous earth was added at a rate of about $10 \mathrm{mg} / \mathrm{L}$ as a filter aid, and the water routed through one of the diatomaceous-earth filters. Tracers were added downstream from the filter, and their concentration in the injection stream was adjusted by monitoring the injection water as it ran to waste. The test was started by quick-connecting the injection line to the injection pipe in the we11.

The injection pipe consisted of 51-mm diameter plastic pipe extending to a depth of $43.6 \mathrm{~m}$ in the well. The pipe was equipped with a spring-loaded foot valve to maintain sufficient back pressure to prevent the pressure in the injection pipe at land surface from decreasing to less than that in the atmosphere. Such an arrangement prevents air from entering the injection stream at joints or valves, becoming entrained, and clogging the aquifer near the well bore.

The planned rate of injection for the March test was $10 \mathrm{~L} / \mathrm{s}$, based on analyses of data from the March 1977 pumping test; however, the maximum longterm pumping rate for well 3 was only $3.8 \mathrm{~L} / \mathrm{s}$. Consequently, water was pumped at that rate from well 3 into the horseshoe-shaped settling basin for 7 days prior to the start of the test to provide a supply reservoir. However, leakage from the settling basin about equalled the $3.8 \mathrm{~L} / \mathrm{s}$ inflow rate when about $1,200 \mathrm{~m}^{3}$ of water was in storage and this was the maximum volume that could be stored. The stored water plus the well 3 pumpage was adequate for about $24 \mathrm{~h}$ of injection at the planned rate.

During the first hour of the test, an attempt was made to maintain the injection rate constant at $10 \mathrm{~L} / \mathrm{s}$ by manually adjusting a gate valve on the injection line. However, because of the damped response of the in-line flowmeter recorder and a rapid rise of water level in the injection we11 (which caused a back pressure that affected the injection rate), the flow rate varied somewhat during the first hour. Moreover, the water level in the injection well had risen about $26 \mathrm{~m}$ during that hour, and it became obvious 
Table 8.--Injection rates and cumulative volumes for the March 1-2, 1978, injection test

\begin{tabular}{|c|c|c|c|c|c|c|}
\hline Date and & Flow rate & & & Recharge & volume & \\
\hline interval & $(\mathrm{L} / \mathrm{s})$ & $\begin{array}{l}\text { ment } \\
\text { (min) }\end{array}$ & $\begin{array}{l}\text { time } \\
\text { (min) }\end{array}$ & $\begin{array}{c}\text { Incremental } \\
\text { (L) }\end{array}$ & $\begin{array}{c}\text { Cumulative } \\
\text { (L) }\end{array}$ & Comment \\
\hline \multicolumn{7}{|l|}{$3 / 1 / 78:$} \\
\hline $1200-1210$ & 9.2 & 10 & 10 & 5,500 & 5,500 & \\
\hline $1210-1214$ & 10.0 & 4 & 14 & 2,400 & 7,900 & \\
\hline $1214-1300$ & 9.5 & 46 & 60 & 26,200 & 34,100 & \\
\hline $1300-0020$ & 7.5 & 680 & 740 & 306,300 & 340,400 & \\
\hline \multicolumn{7}{|l|}{$3 / 2 / 78:$} \\
\hline $0020-0240$ & 7.6 & 140 & 880 & 64,100 & 404,500 & \\
\hline $0240-0355$ & 7.4 & 75 & 955 & 33,200 & 437,700 & \\
\hline $0355-0727$ & 6.5 & 212 & 1,167 & 82,600 & 520,300 & \\
\hline $0727-0800$ & 3.5 & 33 & 1,200 & 6,900 & 527,200 & $\begin{array}{l}\text { Water } \\
\text { level in } \\
\text { settling } \\
\text { basin } \\
\text { below } \\
\text { pump } \\
\text { intake }\end{array}$ \\
\hline $0800-1000$ & 5.7 & 120 & 1,320 & 40,900 & 568,100 & \\
\hline
\end{tabular}


that water would flow from the well in about another hour at that injection rate. Consequently, the injection rate was decreased to $7.5 \mathrm{~L} / \mathrm{s}$. This rate was approximately maintained until 0355 on March 2, when it again had to be decreased to prevent the well from overflowing. The rate inadvertently decreased to about $3.5 \mathrm{~L} / \mathrm{s}$ for about $30 \mathrm{~min}$ on March 2, when the facility was unattended, and the water level in the basin declined below the pump intake. At the time the pump intake was adjusted, the flow rate was again decreased (to $5.7 \mathrm{~L} / \mathrm{s}$ ) to prevent the well from overflowing. By 1000, the water leve1 in the injection well was at land surface, and the test was stopped. Flow rates and cumulative volumes of injected water are listed in table 8 .

The approximate $33-\mathrm{m}$ rise of the water leve1 in the injection we11, compared to a maximum water-level rise of about $2 \mathrm{~m}$ in the observation we11 and piezometers located $2 \mathrm{~m}$ from the injection we11, indicated that the injection well became severely plugged during the test. Moreover, it appeared likely that initial development attempts had been inadequate. Causes of the plugging are described in the section on the May test.

\section{Hydraulic Data}

\section{F1owmeter Logs}

In addition to the injection rate into the we11, deep-wel1 flowmeter logs were made periodically to determine the zones within the aquifer that were being recharged. The results of these logs are shown in figure 12 .

\section{Water-leve1 Data and Analysis}

Water levels were closely monitored using steel tapes during the March 1-2 injection test in piezometers 2 and 3 at $2 \mathrm{~m}$, and in piezometers 1 through 4 at both 5 and $30 \mathrm{~m}$. Water levels in the observation wells at 5 and $30 \mathrm{~m}$ could not be measured, because the steel tapes would not pass by the sampling pumps. However, water leve1 in the $2-\mathrm{m}$ observation we 11 rose to above the top of the pump in that well after about $30 \mathrm{~min}$ of injection, and water levels were obtained after that time.

Results of analyses of the water-level buildup data using the Cooper and Jacob (1946) modification of the Theis (1935) non-equilibrium equation are listed in table 9. For these analyses, data collected from 100 to $1,000 \mathrm{~min}$ after the start of injection were analyzed. These data were selected because the injection rate was relatively stable, and the decrease in the rate after 60 min of injection had become small. In general, the results are quite similar to those obtained from the March 1977 pumping test. However, computed values for the storage coefficient varied over an even greater range. This probably results both from the quasi-partial penetration effects described above, and from the effects of clogging during injection. 

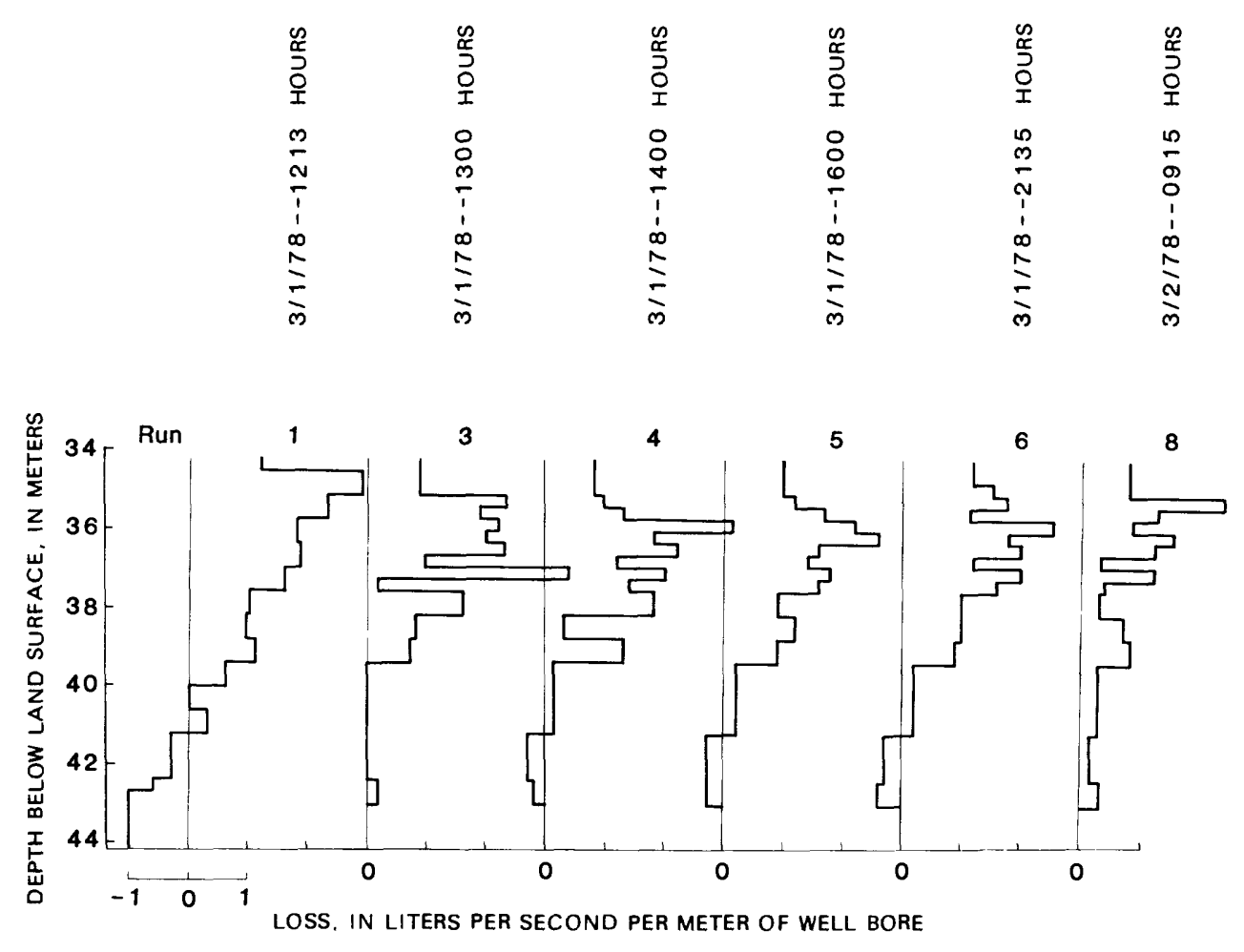

Figure 12.--Flowmeter logs from the injection well obtained during the March 1978 test. 
Table 9.--Transmissivity and storage values computed for the Ogallala aquifer, based on data from the March 1978 injection test

[Computations are based on data obtained from 100 to 1,000 min, using an injection rate of $7.57 \mathrm{~L} / \mathrm{s}$ ]

\begin{tabular}{cccc}
\hline $\begin{array}{c}\text { We11 } \\
\text { number }\end{array}$ & $\begin{array}{c}\text { Distance from } \\
\text { production wel1 } \\
(\mathrm{m})\end{array}$ & $\begin{array}{c}\text { Transmissivity } \\
\left(\mathrm{m}^{2} / \mathrm{d}\right)\end{array}$ & $\begin{array}{c}\text { Storage } \\
\text { Coefficient } \\
\text { (dimensionless) }\end{array}$ \\
\hline I-1 & 2 & 300 & $2 \times 10^{-5}$ \\
I-2-3 & 2 & 210 & $1 \times 10^{-3}$ \\
I-2-2 & 2 & 210 & $4 \times 10^{-4}$ \\
I-6-4 & 5 & 260 & $3 \times 10^{-2}$ \\
I-6-3 & 5 & 240 & $7 \times 10^{-3}$ \\
I-6-2 & 5 & 230 & $8 \times 10^{-4}$ \\
A-10 & 15 & 280 & $4 \times 10^{-3}$ \\
I-8-4 & 30 & 230 & $1 \times 10^{-2}$ \\
I-8-3 & 30 & 240 & $8 \times 10^{-3}$ \\
I-8-2 & 30 & 290 & $3 \times 10^{-2}$ \\
I-8-1 & 30 & 290 & $3 \times 10^{-2}$ \\
\hline
\end{tabular}


Availability of flowmeter and water-level data from various piezometers make feasible the use of computer simulation to identify, at least grossly, the vertical hydraulic-conductivity distribution in the aquifer. However, the results of these simulations are beyond the scope of this report.

\section{Sampling Schedule and Tracer Data}

The injection test began at 1200 on March 1, 1978; sampling of ground water and monitoring of water levels began at that time. Water samples were collected from the 2-m wells at approximately 5-min intervals for the first hour, 10-min intervals for the next $1 \frac{1}{2} \mathrm{~h}$, then increased to $30 \mathrm{~min}, 1 \mathrm{~h}$, and longer intervals for the remainder of the test. Sampling of the 5-m wells began with 10-min sampling intervals and increased in a similar schedule to that for the 2-m wells. The 10-, 15-, and 30-m wells were sampled less frequently, because of the long time required for the tracer to reach those wells.

Two separate tracer experiments were conducted during the injection test. The first experiment began with the start of injection at 1200 on March 1. Iodide, ethanol, and $\mathrm{CBrClF}_{2}$ were added to the injection water according to the schedule shown in table 7. At 2130 on March 1 , the second experiment began with ethano1, benzoate, and Freon-12 used as tracers.

In the following tables, data from the observation wells will be denoted by referring to the well distance from the injection we11. For example, the we11 at $2 \mathrm{~m}$ has the heading "2-Meter we11." Data from the point samplers are titled with a heading that denotes both the radial distance and the color of the point-sampler tubing, which in turn indicates the depth from which the sample was obtained, for example, "2-Meter red point sampler." A key for the depths of point samplers is provided in table 2 , and the spatial arrangement also is shown in figures 4 and 5 .

The breakthrough of ethanol during the first experiment was measured as DOC. The DOC was equivalent to ethanol, because ethanol was the only organic tracer added. The breakthrough of ethanol during the second experiment as DOC was determined by subtracting benzoate as DOC from total DOC. The background concentration of DOC in the Ogallala aquifer water is normally $1.0 \mathrm{mg} / \mathrm{L}$. This background was subtracted from the total DOC for the first experiment. In the second experiment, the background was slightly increased due to the presence of ethanol still in the system from the first experiment. No attempt was made to correct the ethanol measurements in the second experiment for this interference. Analyses of water samples taken from the sampling locations during the first tracer experiment are given in tables 10 to 21 , and from the second tracer experiment in tables 22 to 29. 
Table 10.--Data obtained from the first tracer experiment on March 1, 1978, at the Stanton, Tex. site

[Samples are from the observation well located $2 \mathrm{~m}$ from the injection well.

The 2-m well is screened for the full-saturated thickness of the aquifer.

The dash (-) indicates sample is available but was not analyzed; an asterisk (*) indicates the sample was not analyzed and is no longer available.]

\begin{tabular}{|c|c|c|c|c|c|}
\hline \multicolumn{6}{|c|}{ 2-Meter wel1 } \\
\hline Date & Clock time & $\begin{array}{c}\text { Elapsed time } \\
\text { (min) }\end{array}$ & $\begin{array}{l}\text { Iodide } \\
\text { (mg/L) }\end{array}$ & $\begin{array}{l}\text { Ethano1 } \\
\text { (mg C/L) }\end{array}$ & $\begin{array}{l}\mathrm{CBrC1F}_{2} \\
(\mathrm{ng} / \mathrm{L})\end{array}$ \\
\hline $3-1-78$ & 1200 & 0 & 0.02 & 0 & $*$ \\
\hline Do. & 1205 & 5 & .02 & 0 & $*$ \\
\hline Do. & 1208 & 8 & .02 & 0 & $*$ \\
\hline Do. & 1210 & 10 & -- & 0 & $*$ \\
\hline Do. & 1211 & 11 & .02 & 0 & $*$ \\
\hline Do. & 1213 & 13 & .06 & 0 & 40.0 \\
\hline Do. & 1215 & 15 & .36 & 25 & 125 \\
\hline Do. & 1219 & 19 & .51 & $*$ & 221 \\
\hline Do. & 1220 & 20 & --- & 25 & $*$ \\
\hline Do. & 1222 & 22 & .38 & $*$ & 177 \\
\hline Do. & 1227 & 27 & .33 & $*$ & 125 \\
\hline Do. & 1230 & 30 & --- & 16 & $*$ \\
\hline Do. & 1232 & 32 & .33 & $*$ & 113 \\
\hline Do. & 1235 & 35 & -- & 14 & $*$ \\
\hline Do. & 1237 & 37 & .32 & $*$ & $*$ \\
\hline Do. & 1243 & 43 & .37 & $*$ & 121 \\
\hline Do. & 1248 & 48 & -- & 10 & $*$ \\
\hline Do. & 1250 & 50 & .43 & $*$ & 178 \\
\hline Do. & 1300 & 60 & -- & 14 & 178 \\
\hline Do. & 1310 & 70 & .51 & 17 & 190 \\
\hline Do. & 1320 & 80 & .56 & 15 & $*$ \\
\hline
\end{tabular}


Table 10.--Data obtained from the first tracer experiment on March 1, 1978, at the Stanton, Tex. site--Continued

\begin{tabular}{|c|c|c|c|c|c|}
\hline \multicolumn{6}{|c|}{ 2-Meter we11 } \\
\hline Date & Clock time & $\begin{array}{c}\text { Elapsed time } \\
\text { (min) }\end{array}$ & $\begin{array}{l}\text { Iodide } \\
(\mathrm{mg} / \mathrm{L})\end{array}$ & $\begin{array}{l}\text { Ethanol } \\
\text { (mg C/L) }\end{array}$ & $\begin{array}{l}\mathrm{CBrClF}_{2} \\
(\mathrm{ng} / \mathrm{L})\end{array}$ \\
\hline $3-1-78$ & 1322 & 82 & .60 & $*$ & 217 \\
\hline Do. & 1334 & 94 & .73 & * & 210 \\
\hline Do. & 1340 & 100 & -- & 10 & $*$ \\
\hline Do. & 1349 & 109 & 0.80 & $*$ & 174 \\
\hline Do. & 1400 & 120 & .88 & 8 & 190 \\
\hline Do. & 1420 & 140 & .94 & $*$ & 174 \\
\hline Do. & 1430 & 150 & .95 & 6 & 182 \\
\hline Do. & 1445 & 165 & .97 & $*$ & $*$ \\
\hline Do. & 1500 & 180 & .98 & 6 & $*$ \\
\hline Do. & 1515 & 195 & 1.04 & $*$ & 178 \\
\hline Do. & 1530 & 210 & 1.08 & 5 & $*$ \\
\hline Do. & 1550 & 230 & 1.10 & $*$ & 153 \\
\hline Do. & 1630 & 270 & --- & 2 & $*$ \\
\hline Do. & 1730 & 330 & 1.20 & $*$ & 158 \\
\hline Do. & 1830 & 390 & 1.18 & $*$ & $*$ \\
\hline Do. & 1930 & 450 & 1.20 & $*$ & $*$ \\
\hline Do. & 2135 & 575 & 1.26 & 1 & 53.0 \\
\hline $3-2-78$ & 0815 & 1,215 & 1.30 & $1 /$ & $*$ \\
\hline
\end{tabular}

I/ Interference due to the reuse of this tracer in the second test conducted at 2130 on March 1, 1978 (see subsequent tables). 
Table 11.--Data obtained from the first tracer experiment on March 1, 1978, at the Stanton, Tex. site

[Samples are from the point sampler designated 2-m black located $41.6 \mathrm{~m}$ below land surface and $2 \mathrm{~m}$ radially from the injection well. An asterisk (*) indicates the sample was not analyzed and is no longer available.]

\begin{tabular}{|c|c|c|c|c|c|}
\hline \multicolumn{6}{|c|}{ 2-Meter black point sampler } \\
\hline Date & Clock time & $\begin{array}{c}\text { Elapsed time } \\
\text { (min) }\end{array}$ & $\begin{array}{l}\text { Iodide } \\
(\mathrm{mg} / \mathrm{L})\end{array}$ & $\begin{array}{l}\text { Ethanol } \\
\text { (mg C/L) }\end{array}$ & $\begin{array}{l}\mathrm{CBrClF}_{2} \\
(\mathrm{ng} / \mathrm{L})\end{array}$ \\
\hline $3-1-78$ & 1200 & 0 & 0.07 & 0 & $*$ \\
\hline Do. & 1205 & 5 & .07 & 0 & * \\
\hline Do. & 1211 & 11 & .07 & 0 & $*$ \\
\hline Do. & 1300 & 60 & .06 & 0 & * \\
\hline Do. & 1400 & 120 & .06 & 0 & * \\
\hline Do. & 1500 & 180 & .06 & 0 & $*$ \\
\hline Do. & 2000 & 480 & .11 & 0 & $*$ \\
\hline Do. & 2305 & 665 & .17 & 0 & $*$ \\
\hline $3-2-78$ & 0030 & 750 & .26 & 0 & $*$ \\
\hline Do. & 0100 & 780 & .28 & 0 & 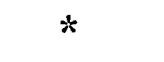 \\
\hline Do. & 0130 & 810 & .31 & 0 & $*$ \\
\hline Do. & 0230 & 870 & .34 & 0 & $*$ \\
\hline Do. & 0400 & 960 & .39 & 0 & $*$ \\
\hline Do. & 0800 & 1,200 & .52 & 0 & $*$ \\
\hline Do. & 1150 & 1,430 & .56 & 0 & $*$ \\
\hline
\end{tabular}


Table 12.--Data obtained from the first tracer experiment on March 1, 1978, at the Stanton, Tex. site

[Samples are from the point sampler designated 2-m red located $2 \mathrm{~m}$ radially from the injection well and $38.7 \mathrm{~m}$ below land surface. The dash (-) indicates sample is available but was not analyzed; an asterisk (*) indicates the sample was not analyzed and is no longer available.]

\begin{tabular}{|c|c|c|c|c|c|}
\hline \multicolumn{6}{|c|}{ 2-Meter red point sampler } \\
\hline Date & Clock time & $\begin{array}{c}\text { Elapsed time } \\
\text { (min) }\end{array}$ & $\begin{array}{l}\text { Iodide } \\
(\mathrm{mg} / \mathrm{L})\end{array}$ & $\begin{array}{l}\text { Ethano1 } \\
(\mathrm{mg} \mathrm{C/L})\end{array}$ & $\begin{array}{r}\mathrm{CBrClF}_{2} \\
(\mathrm{ng} / \mathrm{L})\end{array}$ \\
\hline $3-1-78$ & 1200 & 0 & 0.08 & 0 & $*$ \\
\hline Do. & 1205 & 5 & .06 & 0 & $*$ \\
\hline Do. & 1206 & 6 & ---- & 1 & $*$ \\
\hline Do. & 1208 & 8 & .07 & $*$ & $*$ \\
\hline Do. & 1211 & 11 & .07 & $*$ & $*$ \\
\hline Do. & 1215 & 15 & .07 & 2 & $*$ \\
\hline Do. & 1220 & 20 & .09 & $*$ & $*$ \\
\hline Do. & 1224 & 24 & .11 & $*$ & $*$ \\
\hline Do. & 1225 & 25 & --- & 5.5 & $*$ \\
\hline Do. & 1227 & 27 & .13 & $*$ & $*$ \\
\hline Do. & 1230 & 30 & ---- & 10 & $*$ \\
\hline Do. & 1232 & 32 & .17 & $*$ & $*$ \\
\hline Do. & 1235 & 35 & $-\cdots$ & 15 & $*$ \\
\hline Do. & 1237 & 37 & .20 & $*$ & $*$ \\
\hline Do. & 1240 & 40 & .22 & 21 & $*$ \\
\hline Do. & 1243 & 43 & .26 & $*$ & $*$ \\
\hline Do. & 1248 & 48 & ---- & 31 & $*$ \\
\hline Do. & 1250 & 50 & .32 & $*$ & $*$ \\
\hline Do. & 1255 & 55 & .36 & 33 & $*$ \\
\hline Do. & 1300 & 60 & .40 & 36 & $*$ \\
\hline Do. & 1310 & 70 & .43 & 37 & $*$ \\
\hline Do. & 1315 & 75 & --- & 33 & $*$ \\
\hline Do. & 1320 & 80 & .46 & $*$ & $*$ \\
\hline
\end{tabular}


Table 12.--Data obtained from the first tracer experiment on March 1, 1978, at the Stanton, Tex. site--Continued

\begin{tabular}{|c|c|c|c|c|c|}
\hline \multicolumn{6}{|c|}{ 2-Meter red point sampler } \\
\hline Date & Clock time & $\begin{array}{c}\text { Elapsed time } \\
\text { (min) }\end{array}$ & $\begin{array}{l}\text { Iodide } \\
(\mathrm{mg} / \mathrm{L})\end{array}$ & $\begin{array}{l}\text { Ethanol } \\
(\mathrm{mg} \mathrm{C} / \mathrm{L})\end{array}$ & $\begin{array}{r}\mathrm{CBrClF}_{2} \\
(\mathrm{ng} / \mathrm{L})\end{array}$ \\
\hline $3-1-78$ & 1330 & 90 & 0.59 & 26 & $*$ \\
\hline Do. & 1340 & 100 & .63 & 20 & $*$ \\
\hline Do. & 1349 & 109 & .66 & $*$ & $*$ \\
\hline Do. & 1350 & 110 & ---- & 17 & $*$ \\
\hline Do. & 1400 & 120 & .74 & 16 & $*$ \\
\hline Do. & 1415 & 135 & .80 & 12 & $*$ \\
\hline Do. & 1430 & 150 & .86 & 11 & $*$ \\
\hline Do. & 1445 & 165 & .90 & 10.5 & $*$ \\
\hline Do. & 1500 & 180 & .97 & 9 & $*$ \\
\hline Do. & 1530 & 210 & ---- & 6 & $*$ \\
\hline Do. & 1600 & 240 & ---- & 4.5 & $*$ \\
\hline Do. & 1700 & 300 & ---- & 1.5 & $*$ \\
\hline
\end{tabular}


Table 13.--Data obtained from the first tracer experiment on March 1, 1978, at the Stanton, Tex. site

[Samples are from the point sampler designated 2-m orange located $34.8 \mathrm{~m}$ below land surface and $2 \mathrm{~m}$ radially from the injection well. The dash (-) indicates sample is available but was not analyzed; an asterisk (*) indicates the sample was not analyzed and is no longer available.]

\begin{tabular}{|c|c|c|c|c|c|}
\hline \multicolumn{6}{|c|}{ 2-Meter orange point sampler } \\
\hline Date & Clock time & $\begin{array}{c}\text { Elapsed time } \\
(\min )\end{array}$ & $\begin{array}{l}\text { Iodide } \\
(\mathrm{mg} / \mathrm{L})\end{array}$ & $\begin{array}{l}\text { Ethano1 } \\
(\mathrm{mg} \mathrm{C} / \mathrm{L})\end{array}$ & $\begin{array}{r}\mathrm{CBrC} \mathrm{F}_{2} \\
(\mathrm{ng} / \mathrm{L})\end{array}$ \\
\hline $3-1-78$ & 1200 & 0 & 0.21 & 0 & $*$ \\
\hline Do. & 1205 & 5 & .17 & 0 & $*$ \\
\hline Do. & 1206 & 6 & --- & 1 & $*$ \\
\hline Do. & 1208 & 8 & .15 & $*$ & $*$ \\
\hline Do. & 1211 & 11 & .13 & $*$ & $*$ \\
\hline Do. & 1215 & 15 & --- & 1 & $*$ \\
\hline Do. & 1220 & 20 & .08 & $*$ & $*$ \\
\hline Do. & 1224 & 24 & .09 & $*$ & * \\
\hline Do. & 1225 & 25 & --- & 3 & $*$ \\
\hline Do. & 1227 & 27 & .14 & $*$ & $*$ \\
\hline Do. & 1230 & 30 & --- & 3 & $*$ \\
\hline Do. & 1232 & 32 & .25 & $*$ & $*$ \\
\hline Do. & 1235 & 35 & --- & 4 & $*$ \\
\hline Do. & 1237 & 37 & .40 & $*$ & $*$ \\
\hline Do. & 1240 & 40 & ---- & $*$ & $*$ \\
\hline Do. & 1243 & 43 & .52 & $*$ & $*$ \\
\hline Do. & 1248 & 48 & ---- & 10 & $*$ \\
\hline Do. & 1250 & 50 & .74 & $*$ & $*$ \\
\hline Do. & 1255 & 55 & --- & * & $*$ \\
\hline Do. & 1258 & 58 & .79 & $*$ & $*$ \\
\hline Do. & 1300 & 60 & .85 & 15 & $*$ \\
\hline Do. & 1310 & 70 & .87 & 17 & $*$ \\
\hline Do. & 1315 & 75 & --- & 18 & $*$ \\
\hline
\end{tabular}


Table 13.--Data obtained from the first tracer experiment on March 1, 1978, at the Stanton, Tex. site--Continued

\begin{tabular}{|c|c|c|c|c|c|}
\hline \multicolumn{6}{|c|}{ 2-Meter orange point sampler } \\
\hline Date & Clock time & $\begin{array}{c}\text { Elapsed time } \\
(\min )\end{array}$ & $\begin{array}{l}\text { Iodide } \\
(\mathrm{mg} / \mathrm{L})\end{array}$ & $\begin{array}{l}\text { Ethanol } \\
\text { (mg C/L) }\end{array}$ & $\begin{array}{r}\mathrm{CBrClF}_{2} \\
(\mathrm{ng} / \mathrm{L})\end{array}$ \\
\hline $3-1-78$ & 1320 & 80 & 0.88 & $*$ & $*$ \\
\hline Do. & 1330 & 90 & .91 & 22 & $*$ \\
\hline Do. & 1340 & 100 & .98 & 22 & $*$ \\
\hline Do. & 1349 & 109 & 1.02 & $*$ & $*$ \\
\hline Do. & 1350 & 110 & ---- & 22 & $*$ \\
\hline Do. & 1400 & 120 & .97 & 22 & $*$ \\
\hline Do. & 1415 & 135 & 1.00 & 15 & $*$ \\
\hline Do. & 1430 & 150 & 1.04 & 15 & $*$ \\
\hline Do. & 1445 & 165 & 1.06 & 15 & $*$ \\
\hline Do. & 1500 & 180 & 1.14 & 15 & $*$ \\
\hline Do. & 1530 & 210 & ---- & 8 & $*$ \\
\hline Do. & 1600 & 240 & 1.15 & 6 & $*$ \\
\hline Do. & 1700 & 300 & 1.25 & 2 & $*$ \\
\hline Do. & 1900 & 420 & ---- & 2 & $*$ \\
\hline $3-2-78$ & 0005 & 725 & 1.38 & $1 /$ & $*$ \\
\hline Do. & 0130 & 810 & 1.38 & $1 /$ & $*$ \\
\hline Do. & 0230 & 870 & 1.38 & $1 /$ & $*$ \\
\hline Do. & 0400 & 960 & ---- & $1 /$ & $*$ \\
\hline Do. & 0600 & 1,080 & 1.38 & $1 /$ & $*$ \\
\hline Do. & 0800 & 1,200 & 1.50 & $1 /$ & $*$ \\
\hline Do. & 1150 & 1,430 & 1.70 & $1 /$ & $*$ \\
\hline
\end{tabular}

I/ Interference due to the reuse of this tracer in the second test conducted at 2130 on March 1, 1978 (see subsequent tables). 
Table 14.--Data obtained from the first tracer experiment on March 1, 1978, at the Stanton, Tex. site

[Samples are from the point sampler designated 2-m green located $2 \mathrm{~m}$ radially from the injection well and $32.6 \mathrm{~m}$ below land surface. The dash (-) indicates sample is available but was not analyzed; an asterisk (*) indicates the sample was not analyzed and is no longer available.]

\begin{tabular}{|c|c|c|c|c|c|}
\hline \multicolumn{6}{|c|}{ 2-Meter green point sampler } \\
\hline Date & Clock time & $\begin{array}{c}\text { Elapsed time } \\
(\min )\end{array}$ & $\begin{array}{l}\text { Iodide } \\
(\mathrm{mg} / \mathrm{L})\end{array}$ & $\begin{array}{l}\text { Ethanol } \\
(\mathrm{mg} \mathrm{C} / \mathrm{L})\end{array}$ & $\begin{array}{r}\mathrm{CBrClF}_{2} \\
(\mathrm{ng} / \mathrm{L})\end{array}$ \\
\hline $3-1-78$ & 1200 & 0 & 0.17 & 0 & $*$ \\
\hline Do. & 1204 & 4 & .19 & 0 & $*$ \\
\hline Do. & 1208 & 8 & .21 & 0 & $*$ \\
\hline Do. & 1212 & 12 & .23 & 0 & $*$ \\
\hline Do. & 1216 & 16 & .27 & 0 & $*$ \\
\hline Do. & 1220 & 20 & .31 & 0 & $*$ \\
\hline Do. & 1224 & 24 & .32 & 0 & $*$ \\
\hline Do. & 1228 & 28 & .34 & 0 & $*$ \\
\hline Do. & 1232 & 32 & .34 & $*$ & $*$ \\
\hline Do. & 1236 & 36 & .34 & $*$ & $*$ \\
\hline Do. & 1240 & 40 & .32 & $*$ & $*$ \\
\hline Do. & 1244 & 44 & .32 & $*$ & $*$ \\
\hline Do. & 1245 & 45 & --- & 4 & $*$ \\
\hline Do. & 1248 & 48 & .36 & $*$ & $*$ \\
\hline Do. & 1255 & 55 & .37 & $*$ & $*$ \\
\hline Do. & 1300 & 60 & .38 & 4.5 & $*$ \\
\hline Do. & 1310 & 70 & .39 & 4 & $*$ \\
\hline Do. & 1320 & 80 & .41 & $*$ & $*$ \\
\hline Do. & 1330 & 90 & .42 & $*$ & $*$ \\
\hline Do. & 1340 & 100 & .48 & 4 & $*$ \\
\hline Do. & 1350 & 110 & .53 & 4.5 & $*$ \\
\hline Do. & 1400 & 120 & .55 & 4.5 & $*$ \\
\hline Do. & 1415 & 135 & .60 & 5 & $*$ \\
\hline Do. & 1430 & 150 & .64 & 9 & $*$ \\
\hline
\end{tabular}


Table 14.--Data obtained from the first tracer experiment on March 1, 1978, at the Stanton, Tex. site--Continued

\begin{tabular}{cccccc}
\hline \multicolumn{7}{c}{ 2-Meter green point sampler } & & \\
\hline Date & Clock time & $\begin{array}{c}\text { Elapsed time } \\
(\mathrm{min})\end{array}$ & $\begin{array}{c}\text { Iodide } \\
(\mathrm{mg} / \mathrm{L})\end{array}$ & $\begin{array}{c}\text { Ethanol } \\
(\mathrm{mg} \mathrm{C} / \mathrm{L})\end{array}$ & $\begin{array}{c}\text { CBrC1F } \\
2 \\
(\mathrm{ng} / \mathrm{L})\end{array}$ \\
\hline $3-1-78$ & 1445 & 165 & 0.68 & 9 & $*$ \\
Do. & 1530 & 210 & .82 & $*$ & $*$ \\
Do. & 1600 & 240 & .93 & 10 & $*$ \\
Do. & 1700 & 300 & 1.08 & $*$ & $*$ \\
Do. & 1800 & 360 & 1.20 & 3 & $*$ \\
Do. & 1900 & 420 & 1.26 & 3 & $*$ \\
3-2-78 & 0005 & 725 & 1.43 & $1 /$ & $*$ \\
Do. & 1150 & 1,430 & 1.46 & $-1 /$ & $*$ \\
\hline
\end{tabular}

I/ Interference due to the reuse of this tracer in the second test conducted at 2130 on March 1, 1978 (see subsequent tables). 
Table 15.--Data obtained from the first tracer experiment on March 1, 1978, at the Stanton, Tex. site

[Samples are from the observation well located $5 \mathrm{~m}$ from the injection well. The 5-m well is screened for the full-saturated thickness of the aquifer. The dash (-) indicates sample is available but was not analyzed; an asterisk (*) indicates the sample was not analyzed and is no longer available.]

\begin{tabular}{|c|c|c|c|c|c|}
\hline \multicolumn{6}{|c|}{ 5-Meter well } \\
\hline Date & Clock time & $\begin{array}{c}\text { Elapsed time } \\
\text { (min) }\end{array}$ & $\begin{array}{l}\text { Iodide } \\
(\mathrm{mg} / \mathrm{L})\end{array}$ & $\begin{array}{l}\text { Ethanol } \\
(\mathrm{mg} \mathrm{C} / \mathrm{L})\end{array}$ & $\begin{array}{r}\mathrm{CBrC}_{2} \mathrm{~F}_{2} \\
(\mathrm{ng} / \mathrm{L})\end{array}$ \\
\hline $3-1-78$ & 1200 & 0 & $<0.01$ & $*$ & $*$ \\
\hline Do. & 1213 & 13 & $<.01$ & $*$ & $*$ \\
\hline Do. & 1220 & 20 & $<.01$ & $*$ & * \\
\hline Do. & 1227 & 27 & $<.01$ & $*$ & 2.3 \\
\hline Do. & 1237 & 37 & $<.01$ & $*$ & $*$ \\
\hline Do. & 1240 & 40 & .01 & * & 6.9 \\
\hline Do. & 1243 & 43 & .02 & $*$ & 3.9 \\
\hline Do. & 1250 & 50 & .02 & $*$ & 10.3 \\
\hline Do. & 1300 & 60 & .03 & 0 & 13.7 \\
\hline Do. & 1310 & 70 & .05 & 0 & 18.4 \\
\hline Do. & 1320 & 80 & .06 & 0 & 20.7 \\
\hline Do. & 1330 & 90 & .09 & 0 & 30.4 \\
\hline Do. & 1349 & 109 & .16 & $*$ & $*$ \\
\hline Do. & 1350 & 110 & --- & 6 & 37.0 \\
\hline Do. & 1400 & 120 & .19 & 6 & 39.5 \\
\hline Do. & 1415 & 135 & .22 & 7.5 & 46.1 \\
\hline Do. & 1430 & 150 & .27 & 8 & 54.0 \\
\hline Do. & 1445 & 165 & .30 & 6 & 53.0 \\
\hline Do. & 1500 & 180 & .30 & $*$ & * \\
\hline Do. & 1515 & 195 & .38 & $*$ & 107 \\
\hline Do. & 1530 & 210 & .39 & $*$ & 113 \\
\hline Do. & 1555 & 235 & $-\infty$ & 8 & $*$ \\
\hline
\end{tabular}


Table 15.--Data obtained from the first tracer experiment on March 1, 1978, at the Stanton, Tex. site--Continued

\begin{tabular}{|c|c|c|c|c|c|}
\hline \multicolumn{6}{|c|}{ 5-Meter we11 } \\
\hline Date & clock time & $\begin{array}{c}\text { Elapsed time } \\
\text { (min) }\end{array}$ & $\begin{array}{l}\text { Iodide } \\
(\mathrm{mg} / \mathrm{L})\end{array}$ & $\begin{array}{l}\text { Ethano1 } \\
(\mathrm{mg} \mathrm{C} / \mathrm{L})\end{array}$ & $\begin{array}{r}\mathrm{CBrClF}_{2} \\
(\mathrm{ng} / \mathrm{L})\end{array}$ \\
\hline $3-1-78$ & 1620 & 260 & .51 & 0 & 149 \\
\hline Do. & 1730 & 330 & .59 & 0 & 135 \\
\hline Do. & 1830 & 390 & 0.66 & 6 & $*$ \\
\hline Do. & 1930 & 450 & .70 & $*$ & $*$ \\
\hline Do. & 2010 & 490 & .72 & $*$ & $*$ \\
\hline Do. & 2135 & 575 & .71 & $*$ & $*$ \\
\hline Do. & 2205 & 605 & --- & $1 /$ & 80.2 \\
\hline Do & 2315 & 675 & --- & $1 /$ & 94.3 \\
\hline Do. & 2415 & 735 & --- & $1 /$ & 70.1 \\
\hline $3-2-78$ & 0130 & 810 & --- & $1 /$ & 35.0 \\
\hline Do. & 0230 & 870 & $-\cdots$ & $1 /$ & 41.0 \\
\hline Do. & 0400 & 960 & --- & 11 & 48.0 \\
\hline Do. & 0600 & 1,080 & .80 & $1 /$ & $*$ \\
\hline Do. & 0630 & 1,110 & --- & $1 /$ & 43.0 \\
\hline Do. & 0800 & 1,200 & .80 & $1 /$ & 38.9 \\
\hline Do. & 0945 & 1,305 & .81 & $1 /$ & $*$ \\
\hline Do. & 1100 & 1,380 & .67 & $1 /$ & 27.9 \\
\hline
\end{tabular}

1/Interference due to the reuse of this tracer in the second test conducted at 2130 on March 1, 1978 (see subsequent tables). 
Table 16.--Data obtained from the first tracer experiment on March 1, 1978, at the Stanton, Tex. site

[Samples are from the point sampler designated 5-m black located $5 \mathrm{~m}$ radially from the injection well and $41.8 \mathrm{~m}$ below land surface. An asterisk (*) indicates the sample was not analyzed and is no longer available.]

\begin{tabular}{|c|c|c|c|c|c|}
\hline \multicolumn{6}{|c|}{ 5-Meter black point sampler } \\
\hline Date & Clock time & $\begin{array}{c}\text { Elapsed time } \\
(\min )\end{array}$ & $\begin{array}{l}\text { Iodide } \\
(\mathrm{mg} / \mathrm{L})\end{array}$ & $\begin{array}{l}\text { Ethanol } \\
(\mathrm{mg} \mathrm{C} / \mathrm{L})\end{array}$ & $\begin{array}{r}\mathrm{CBrClF}_{2} \\
(\mathrm{ng} / \mathrm{L})\end{array}$ \\
\hline $3-1-78$ & 1211 & 0 & 0.13 & 0 & $*$ \\
\hline Do. & 1220 & 9 & .13 & 0 & $*$ \\
\hline Do. & 1230 & 19 & .14 & 0 & $*$ \\
\hline Do. & 1310 & 59 & .15 & 0 & $*$ \\
\hline Do. & 1410 & 119 & .17 & 0 & $*$ \\
\hline Do. & 1550 & 219 & .27 & 0 & $*$ \\
\hline Do. & 1630 & 259 & .32 & 0 & $*$ \\
\hline Do. & 1710 & 299 & .35 & 0 & $*$ \\
\hline Do. & 1800 & 349 & .40 & 0 & $*$ \\
\hline Do. & 1900 & 409 & .45 & 0 & $*$ \\
\hline Do. & 1930 & 439 & .46 & 0 & $*$ \\
\hline Do. & 2000 & 469 & .53 & 0 & $*$ \\
\hline Do. & 2030 & 499 & .59 & 0 & * \\
\hline Do. & 2245 & 634 & .72 & $1 /$ & $*$ \\
\hline $3-2-78$ & 0005 & 714 & 1.01 & $1 /$ & $*$ \\
\hline Do. & 1100 & 1,369 & 1.01 & $1 /$ & $*$ \\
\hline Do. & 1150 & 1,419 & 1.01 & $1 /$ & $*$ \\
\hline
\end{tabular}

I/ Interference due to the reuse of this tracer in the second test conducted at 2130 on March 1, 1978 (see subsequent tables). 
Table 17.--Data obtained from the first tracer experiment on March 1, 1978, at the Stanton, Tex. site

[Samples are from the point sampler designated 5-m orange located $37.5 \mathrm{~m}$ below land surface and $5 \mathrm{~m}$ radially from the injection well. The dash (-) indicates sample is available but was not analyzed; an asterisk (*) indicates the sample was not analyzed and is no longer available.]

\begin{tabular}{|c|c|c|c|c|c|}
\hline \multicolumn{6}{|c|}{ 5-Meter orange point sampler } \\
\hline Date & Clock time & $\begin{array}{c}\text { Elapsed time } \\
\text { (min) }\end{array}$ & $\begin{array}{l}\text { Iodide } \\
(\mathrm{mg} / \mathrm{L})\end{array}$ & $\begin{array}{l}\text { Ethano1 } \\
(\mathrm{mg} \mathrm{C/L})\end{array}$ & $\begin{array}{r}\mathrm{CBrClF}_{2} \\
(\mathrm{ng} / \mathrm{L})\end{array}$ \\
\hline $3-1-78$ & 1211 & 0 & 0.05 & 0 & $*$ \\
\hline Do. & 1230 & 19 & .04 & 0 & $*$ \\
\hline Do. & 1240 & 29 & .04 & 0 & $*$ \\
\hline Do. & 1258 & 47 & .08 & 0 & $*$ \\
\hline Do. & 1305 & 54 & .10 & 0 & $*$ \\
\hline Do. & 1317 & 66 & --- & 4 & $*$ \\
\hline Do. & 1320 & 69 & .18 & $*$ & $*$ \\
\hline Do. & 1322 & 71 & .29 & $*$ & $*$ \\
\hline Do. & 1325 & 74 & --- & 6 & $*$ \\
\hline Do & 1330 & 79 & --- & 8 & $*$ \\
\hline Do. & 1334 & 83 & .38 & $*$ & $*$ \\
\hline Do. & 1349 & 98 & .41 & $*$ & $*$ \\
\hline Do. & 1400 & 109 & ---- & 12 & $*$ \\
\hline Do. & 1410 & 119 & .41 & $*$ & $*$ \\
\hline Do. & 1430 & 139 & .42 & 12 & $*$ \\
\hline Do. & 1440 & 149 & --- & 12 & $*$ \\
\hline Do. & 1450 & 159 & --- & 12 & $*$ \\
\hline Do. & 1500 & 169 & --- & 12 & $*$ \\
\hline Do. & 1510 & 179 & .51 & 11 & $*$ \\
\hline Do. & 1520 & 189 & --- & 11 & $*$ \\
\hline Do. & 1530 & 199 & .51 & 11 & $*$ \\
\hline Do. & 1540 & 209 & --- & 10 & $*$ \\
\hline Do. & 1550 & 219 & .51 & 11 & $*$ \\
\hline
\end{tabular}


Table 17.--Data obtained from the first tracer experiment on March 1, 1978, at the Stanton, Tex. site--Continued

\begin{tabular}{|c|c|c|c|c|c|}
\hline \multicolumn{6}{|c|}{ 5-Meter orange point sampler } \\
\hline Date & Clock time & $\begin{array}{c}\text { Elapsed time } \\
\text { (min) }\end{array}$ & $\begin{array}{l}\text { Iodide } \\
(\mathrm{mg} / \mathrm{L})\end{array}$ & $\begin{array}{l}\text { Ethanol } \\
\text { (mg C/L) }\end{array}$ & $\begin{array}{r}\mathrm{CBrClF}_{2} \\
(\mathrm{ng} / \mathrm{L})\end{array}$ \\
\hline $3-1-78$ & 1610 & 239 & --- & 10 & $*$ \\
\hline Do. & 1650 & 279 & .54 & $*$ & $*$ \\
\hline Do. & 1730 & 319 & --- & 6 & $*$ \\
\hline Do & 1800 & 349 & 0.49 & $*$ & $*$ \\
\hline Do & 1830 & 379 & --- & 5 & $*$ \\
\hline Do. & 1930 & 439 & .49 & $*$ & $*$ \\
\hline Do. & 2030 & 499 & .53 & $*$ & $*$ \\
\hline Do. & 2245 & 634 & .75 & $1 /$ & $*$ \\
\hline $3-2-78$ & 0005 & 714 & .90 & $1 /$ & $*$ \\
\hline Do . & 1150 & 1,419 & .86 & $1 /$ & $*$ \\
\hline
\end{tabular}

II Interference due to the reuse of this tracer in the second test conducted at 2130 on March 1, 1978 (see subsequent tables). 
Table 18.--Data obtained from the first tracer experiment on March 1, 1978, at the Stanton, Tex. site

[Samples are from the point sampler designated 5-m green located $5 \mathrm{~m}$ radially from the injection well and $33.9 \mathrm{~m}$ below land surface. An asterisk (*) indicates the sample was not analyzed and is no longer available.]

\begin{tabular}{|c|c|c|c|c|c|}
\hline \multicolumn{6}{|c|}{ 5-Meter green point sampler } \\
\hline Date & Clock time & $\begin{array}{c}\text { Elapsed time } \\
\text { (min) }\end{array}$ & $\begin{array}{l}\text { Iodide } \\
(\mathrm{mg} / \mathrm{L})\end{array}$ & $\begin{array}{l}\text { Ethanol } \\
(\mathrm{mg} \mathrm{C} / \mathrm{L})\end{array}$ & $\begin{array}{r}\mathrm{CBrC} \mathrm{F}_{2} \\
(\mathrm{ng} / \mathrm{L})\end{array}$ \\
\hline $3-1-78$ & 1211 & 0 & 0.02 & $*$ & $*$ \\
\hline Do. & 1230 & 19 & .02 & $*$ & $*$ \\
\hline Do. & 1240 & 29 & .04 & $*$ & $*$ \\
\hline Do. & 1250 & 39 & .04 & $\star$ & $*$ \\
\hline Do. & 1255 & 44 & .05 & $*$ & $*$ \\
\hline Do. & 1258 & 47 & .07 & $*$ & $\star$ \\
\hline Do. & 1300 & 49 & .10 & $*$ & $*$ \\
\hline Do. & 1310 & 59 & .12 & $*$ & $*$ \\
\hline Do. & 1320 & 69 & .15 & $*$ & $*$ \\
\hline Do. & 1330 & 79 & .29 & $*$ & $*$ \\
\hline Do. & 1349 & 98 & .29 & $*$ & $*$ \\
\hline Do. & 1410 & 119 & .39 & $*$ & $*$ \\
\hline Do. & 1430 & 139 & .42 & $*$ & $*$ \\
\hline Do. & 1500 & 169 & .55 & $*$ & $*$ \\
\hline Do. & 1530 & 199 & .68 & $*$ & $*$ \\
\hline Do. & 1600 & 229 & .73 & $*$ & $*$ \\
\hline Do. & 1650 & 279 & .71 & $*$ & $*$ \\
\hline Do. & 1730 & 319 & .79 & $*$ & $*$ \\
\hline Do. & 1830 & 379 & .92 & $*$ & $*$ \\
\hline Do. & 1930 & 439 & .82 & $*$ & $*$ \\
\hline Do. & 2030 & 499 & .84 & $*$ & $*$ \\
\hline Do. & 2245 & 634 & .84 & $*$ & $*$ \\
\hline $3-2-78$ & 0005 & 714 & .84 & $*$ & $\star$ \\
\hline
\end{tabular}


Table 19.--Data obtained from the first tracer experiment on March 1, 1978, at the Stanton, Tex. site

[Samples are from the observation well $10 \mathrm{~m}$ from the injection well and are screened only for a $1.2-\mathrm{m}$ section, $41.5 \mathrm{~m}$ below land surface. An asterisk (*) indicates the sample was not analyzed and is no longer available.]

\begin{tabular}{|c|c|c|c|c|c|}
\hline \multicolumn{6}{|c|}{ 10-Meter wel1 } \\
\hline Date & Clock time & $\begin{array}{c}\text { Elapsed time } \\
(\mathrm{min})\end{array}$ & $\begin{array}{l}\text { Iodide } \\
(\mathrm{mg} / \mathrm{L})\end{array}$ & $\begin{array}{l}\text { Ethanol } \\
(\mathrm{mg} \mathrm{C} / \mathrm{L})\end{array}$ & $\begin{array}{r}\mathrm{CBrClF}_{2} \\
(\mathrm{ng} / \mathrm{L})\end{array}$ \\
\hline $3-1-78$ & 1205 & 0 & 0.13 & $\star$ & 0 \\
\hline Do. & 1230 & 25 & .12 & $*$ & $*$ \\
\hline Do. & 1245 & 40 & .12 & $*$ & $*$ \\
\hline Do. & 1300 & 55 & .11 & $*$ & $*$ \\
\hline Do. & 1440 & 155 & $<.1$ & $*$ & $*$ \\
\hline Do. & 1530 & 205 & $<.1$ & $*$ & $*$ \\
\hline Do. & 1625 & 260 & $<.1$ & $*$ & $*$ \\
\hline Do. & 1645 & 280 & $<.1$ & $*$ & $*$ \\
\hline Do. & 1730 & 325 & $<.1$ & $*$ & $*$ \\
\hline Do. & 1830 & 385 & $<.1$ & $*$ & 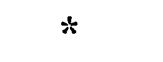 \\
\hline Do. & 1930 & 445 & $<.1$ & $*$ & $*$ \\
\hline Do. & 2010 & 485 & $<.1$ & $*$ & $*$ \\
\hline Do. & 2130 & 565 & $<.1$ & $*$ & 0 \\
\hline Do. & 2230 & 625 & $<.1$ & $*$ & 0 \\
\hline $3-2-78$ & 0130 & 805 & $<.1$ & $*$ & 0 \\
\hline Do. & 0645 & 1,120 & $<.1$ & $*$ & 10.7 \\
\hline Do. & 0820 & 1,215 & $<.1$ & $*$ & 0 \\
\hline Do. & 0958 & 1,313 & $<.1$ & $*$ & 0 \\
\hline Do. & 1010 & 1,325 & $<.1$ & $*$ & 0 \\
\hline
\end{tabular}


Table 20.--Data obtained from the first tracer experiment on March 1, 1978, at the stanton, Tex. site

[Samples are from the observation well $15 \mathrm{~m}$ from the injection well, which is screened for the saturated thickness of the aquifer. The dash (-) indicates sample is available but was not analyzed; an asterisk (*) indicates the sample was not analyzed and is no longer available.]

\begin{tabular}{|c|c|c|c|c|c|}
\hline \multicolumn{6}{|c|}{ 15-Meter well } \\
\hline Date & Clock time & $\begin{array}{c}\text { Elapsed time } \\
(\min )\end{array}$ & $\begin{array}{l}\text { Iodide } \\
(\mathrm{mg} / \mathrm{L})\end{array}$ & $\begin{array}{l}\text { Ethanol } \\
(\mathrm{mg} \mathrm{C} / \mathrm{L})\end{array}$ & $\begin{array}{r}\mathrm{CBrClF}_{2} \\
(\mathrm{ng} / \mathrm{L})\end{array}$ \\
\hline $3-1-78$ & 1215 & 0 & $<0.01$ & $*$ & 0 \\
\hline Do. & 1230 & 15 & $<.01$ & $*$ & * \\
\hline Do. & 1300 & 45 & $<.01$ & $*$ & $*$ \\
\hline Do. & 1330 & 75 & $<.01$ & $*$ & $*$ \\
\hline Do. & 1345 & 90 & $*$ & $*$ & 0 \\
\hline Do. & 1400 & 105 & $<.01$ & $*$ & $*$ \\
\hline Do. & 1445 & 150 & $<.01$ & $*$ & $*$ \\
\hline Do. & 1630 & 255 & $<.01$ & $*$ & $*$ \\
\hline Do. & 1730 & 315 & $<.01$ & $*$ & $*$ \\
\hline Do. & 1830 & 375 & $<.01$ & $*$ & $*$ \\
\hline Do. & 1930 & 435 & $<.01$ & $*$ & $*$ \\
\hline Do. & 2010 & 475 & $<.01$ & $*$ & $*$ \\
\hline Do. & 2135 & 560 & $<.01$ & $*$ & $*$ \\
\hline Do. & 2230 & 625 & $*$ & $*$ & 0 \\
\hline $3-2-78$ & 0335 & 920 & .13 & $*$ & $*$ \\
\hline Do. & 0600 & 1,065 & .20 & $*$ & $*$ \\
\hline Do. & 0640 & 1,105 & $*$ & $*$ & 0 \\
\hline Do. & 0820 & 1,205 & .22 & $*$ & 10.8 \\
\hline Do. & 1145 & 1,425 & --- & $*$ & 1.9 \\
\hline Do. & 1235 & 1,475 & --- & $*$ & 2.8 \\
\hline
\end{tabular}


Table 21.--Data obtained from the first tracer experiment on March 1, 1978, at the stanton, Tex. site

[Samples are from the observation well $30 \mathrm{~m}$ from the injection well, which is screened for the saturated thickness of the aquifer. An asterisk (*) indicates the sample was not analyzed and is no longer available.]

\begin{tabular}{|c|c|c|c|c|c|}
\hline \multicolumn{6}{|c|}{ 30-Meter wel1 } \\
\hline Date & Clock time & $\begin{array}{c}\text { Elapsed time } \\
(\min )\end{array}$ & $\begin{array}{l}\text { Iodide } \\
(\mathrm{mg} / \mathrm{L})\end{array}$ & $\begin{array}{l}\text { Ethanol } \\
(\mathrm{mg} \mathrm{C/L})\end{array}$ & $\begin{array}{r}\mathrm{CBrC} \mathrm{F}_{2} \\
(\mathrm{ng} / \mathrm{L})\end{array}$ \\
\hline $3-1-78$ & 1230 & 0 & $<0.1$ & $*$ & $*$ \\
\hline Do. & 1330 & 60 & $<.1$ & $*$ & $*$ \\
\hline Do. & 1400 & 90 & $<.1$ & $*$ & * \\
\hline Do. & 1530 & 180 & $<.1$ & $*$ & $*$ \\
\hline Do. & 1630 & 240 & $<.1$ & $*$ & $*$ \\
\hline Do. & 1730 & 300 & $<.1$ & $*$ & $*$ \\
\hline Do. & 1830 & 360 & $<.1$ & $*$ & $*$ \\
\hline Do. & 1930 & 420 & $<.1$ & * & $*$ \\
\hline Do. & 2015 & 465 & $<.1$ & $*$ & * \\
\hline Do. & 2135 & 545 & $<.1$ & $\star$ & $*$ \\
\hline Do. & 2230 & 600 & $<.1$ & $*$ & $*$ \\
\hline Do. & 2245 & 615 & $<.1$ & $*$ & $*$ \\
\hline Do. & 2330 & 660 & $<.1$ & $*$ & $*$ \\
\hline $3-2-78$ & 0130 & 780 & $<.1$ & $*$ & $*$ \\
\hline Do. & 0330 & 900 & $<.1$ & $*$ & $*$ \\
\hline Do. & 0620 & 1,070 & $<.1$ & $*$ & $*$ \\
\hline Do. & 0820 & 1,190 & $<.1$ & $*$ & $*$ \\
\hline Do. & 0955 & 1,285 & $<.1$ & $*$ & $*$ \\
\hline
\end{tabular}


Table 22.--Data obtained from the second tracer experiment on March 1, 1978, at the Stanton, Tex. site

[Samples are from the observation well located $2 \mathrm{~m}$ from the injection we11. The 2-m well is screened for the full-saturated thickness of the aquifer. The asterisk $(*)$ indicates the sample was not analyzed and is no longer available.]

\begin{tabular}{|c|c|c|c|c|c|}
\hline \multicolumn{6}{|c|}{ 2-Meter wel1 } \\
\hline Date & Clock time & $\begin{array}{c}\text { Elapsed time } \\
\text { (min) }\end{array}$ & $\begin{array}{l}\text { Ethanol } \\
\text { (mg C/L) }\end{array}$ & $\begin{array}{l}\text { Benzoate } \\
(\mathrm{mg} \mathrm{C} / \mathrm{L} \text { ) }\end{array}$ & $\begin{array}{l}\mathrm{CC}_{2} \mathrm{~F}_{2} \\
(\mathrm{ng} / \mathrm{L})\end{array}$ \\
\hline $3-1-78$ & 2145 & 15 & 1 & 0.00 & 6.59 \\
\hline Do. & 2150 & 20 & 2 & .00 & $*$ \\
\hline Do. & 2155 & 25 & 6 & .23 & $*$ \\
\hline Do. & 2200 & 30 & 6 & .57 & 45.5 \\
\hline Do. & 2205 & 35 & 7 & 1.00 & 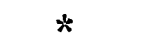 \\
\hline Do. & 2210 & 40 & 11 & 1.27 & $*$ \\
\hline Do. & 2215 & 45 & 11 & 1.47 & $*$ \\
\hline Do. & 2220 & 50 & 11 & 1.63 & $\star$ \\
\hline Do. & 2225 & 55 & 16 & 1.90 & $*$ \\
\hline Do. & 2230 & 60 & 15 & 2.10 & 110 \\
\hline Do. & 2235 & 65 & 17 & 2.40 & $*$ \\
\hline Do. & 2240 & 70 & 17 & 2.57 & $*$ \\
\hline Do. & 2245 & 75 & 21 & $*$ & 123 \\
\hline Do. & 2250 & 80 & 19 & 2.47 & $*$ \\
\hline Do. & 2255 & 85 & 16 & 2.20 & $*$ \\
\hline Do. & 2300 & 90 & 16 & 1.87 & 117 \\
\hline Do. & 2305 & 95 & * & 1.73 & $*$ \\
\hline Do. & 2310 & 100 & 11 & * & $*$ \\
\hline Do . & 2315 & 105 & * & 1.33 & 77 \\
\hline Do. & 2320 & 110 & 8 & $*$ & $*$ \\
\hline Do. & 2325 & 115 & $*$ & 1.07 & 97.3 \\
\hline Do. & 2330 & 120 & 8 & $*$ & $*$ \\
\hline
\end{tabular}


Table 22.--Data obtained from the second tracer experiment on March 1, 1978, at the Stanton, Tex. site--Continued

\begin{tabular}{lccccc}
\hline 2-Meter well & & \\
\hline Date & Clock time & $\begin{array}{c}\text { Elapsed time } \\
\text { (min) }\end{array}$ & $\begin{array}{c}\text { Ethanol } \\
(\mathrm{mg} \mathrm{C} / \mathrm{L})\end{array}$ & $\begin{array}{c}\text { Benzoate } \\
(\mathrm{mg} \mathrm{C} / \mathrm{L})\end{array}$ & $\begin{array}{c}\mathrm{CCl}_{2} \mathrm{~F}_{2} \\
(\mathrm{ng} / \mathrm{L})\end{array}$ \\
\hline $3-1-78$ & 2400 & 150 & 6 & .40 & 73.1 \\
3-2-78 & 0015 & 165 & 5.6 & 0.40 & $*$ \\
Do. & 0030 & 180 & 5.7 & .30 & $*$ \\
Do. & 0100 & 210 & $*$ & .17 & 37.0 \\
Do. & 0130 & 240 & 5.4 & .10 & $*$ \\
Do. & 0200 & 270 & $*$ & 0 & $*$ \\
Do. & 0300 & 330 & $*$ & 0 & $*$ \\
Do. & 0400 & 390 & $*$ & 0 & $*$ \\
Do. & 0630 & 540 & $*$ & 0 & $*$ \\
\hline
\end{tabular}


Table 23.--Data obtained from the second tracer experiment on March 1, 1978, at the Stanton, Tex. site

[Samples are from the point sampler designated 2-m black located $41.6 \mathrm{~m}$ below land surface and $2 \mathrm{~m}$ radially from the injection well. The asterisk $(*)$ indicates the sample was not analyzed and is no longer available.]

\begin{tabular}{cccccc}
\hline \multicolumn{7}{c}{ 2-Meter black point sampler } \\
\hline Date & Clock time & $\begin{array}{c}\text { Elapsed time } \\
(\mathrm{min})\end{array}$ & $\begin{array}{c}\text { Ethanol } \\
(\mathrm{mg} \mathrm{C} / \mathrm{L})\end{array}$ & $\begin{array}{c}\text { Benzoate } \\
(\mathrm{mg} \mathrm{C} / \mathrm{L})\end{array}$ & $\begin{array}{c}\mathrm{CCl}_{2} \mathrm{~F}_{2} \\
(\mathrm{ng} / \mathrm{L})\end{array}$ \\
\hline $3-1-78$ & 2135 & 5 & 0 & 0 & $*$ \\
$3-2-78$ & 0030 & 180 & 0 & 0 & $*$ \\
Do. & 0230 & 300 & 0 & .07 & $*$ \\
Do. & 0400 & 390 & 0 & .10 & $*$ \\
Do. & 0600 & 510 & 0 & .13 & $*$ \\
\hline
\end{tabular}


Table 24.--Data obtained from the second tracer experiment on March 1, 1978, at the stanton, Tex. site

[Samples are from the point sampler designated 2-m red located $2 \mathrm{~m}$ radially from the injection well and $38.7 \mathrm{~m}$ below land surface. The asterisk (*) indicates the sample was not analyzed and is no longer available.]

\begin{tabular}{|c|c|c|c|c|c|}
\hline \multicolumn{6}{|c|}{ 2-Meter red point sampler } \\
\hline Date & Clock time & $\begin{array}{c}\text { Elapsed time } \\
\text { (min) }\end{array}$ & $\begin{array}{l}\text { Ethanol } \\
(\mathrm{mg} \mathrm{C} / \mathrm{L})\end{array}$ & $\begin{array}{l}\text { Benzoate } \\
(\mathrm{mg} \mathrm{C} / \mathrm{L})\end{array}$ & $\begin{array}{l}\mathrm{CCl}_{2} \mathrm{~F}_{2} \\
(\mathrm{ng} / \mathrm{L})\end{array}$ \\
\hline $3-1-78$ & 2130 & 0 & 2 & $\star$ & * \\
\hline Do. & 2140 & 10 & 2 & 0 & * \\
\hline Do. & 2145 & 15 & 2 & 0 & $*$ \\
\hline Do. & 2150 & 20 & 2 & .05 & $*$ \\
\hline Do. & 2155 & 25 & 2 & .2 & $*$ \\
\hline Do. & 2200 & 30 & 3 & .3 & $*$ \\
\hline Do. & 2205 & 35 & 4 & .55 & * \\
\hline Do . & 2210 & 40 & 5 & .65 & $*$ \\
\hline Do. & 2215 & 45 & $*$ & * & * \\
\hline Do. & 2220 & 50 & 8 & .95 & $\star$ \\
\hline Do. & 2225 & 55 & 12 & 1.15 & * \\
\hline Do. & 2230 & 60 & 16 & 1.4 & $*$ \\
\hline Do. & 2235 & 65 & 17 & 1.65 & $*$ \\
\hline Do. & 2240 & 70 & 22 & 1.8 & $*$ \\
\hline Do. & 2245 & 75 & 24 & 1.85 & $*$ \\
\hline Do. & 2250 & 80 & 26 & 1.6 & $*$ \\
\hline Do. & 2255 & 85 & * & 1.55 & * \\
\hline Do. & 2300 & 90 & 28 & 1.8 & $*$ \\
\hline Do. & 2310 & 100 & 25 & 1.35 & $*$ \\
\hline Do. & 2320 & 110 & 20 & 1.6 & $*$ \\
\hline Do. & 2330 & 120 & 12 & 1.55 & $*$ \\
\hline Do. & 2345 & 135 & 7 & 1.1 & $*$ \\
\hline Do. & 2400 & 150 & 2 & .95 & $*$ \\
\hline
\end{tabular}


Table 24.--Data obtained from the second tracer experiment on March 1, 1978, at the Stanton, Tex. site--Continued

\begin{tabular}{lccccc}
\hline \multicolumn{7}{c}{ 2-Meter red point sampler } & \\
\hline Date & Clock time & $\begin{array}{c}\text { Elapsed time } \\
(\mathrm{min})\end{array}$ & $\begin{array}{c}\text { Ethanol } \\
(\mathrm{mg} \mathrm{C} / \mathrm{L})\end{array}$ & $\begin{array}{c}\text { Benzoate } \\
(\mathrm{mg} \mathrm{C} / \mathrm{L})\end{array}$ & $\begin{array}{c}\mathrm{CCl}_{2} \mathrm{~F}_{2} \\
(\mathrm{ng} / \mathrm{L})\end{array}$ \\
\hline 3-2-78 & 0015 & 165 & 1 & .7 & $*$ \\
Do. & 0030 & 180 & 1 & 0.6 & $*$ \\
Do. & 0100 & 210 & 0 & .3 & $*$ \\
Do. & 0130 & 240 & 0 & .3 & $*$ \\
Do. & 0230 & 300 & 0 & .25 & $*$ \\
Do. & 0400 & 390 & 0 & 0 & $*$ \\
Do. & 0600 & 510 & 0 & 0 & $*$ \\
Do. & 0800 & 630 & 0 & & $*$ \\
\hline
\end{tabular}


Table 25.--Data obtained from the second tracer experiment on March 1, 1978, at the stanton, Tex. site

[Samples are from the point sampler designated 2-m orange located $34.8 \mathrm{~m}$ below land surface and $2 \mathrm{~m}$ radially from the injection well. The asterisk (*) indicates the sample was not analyzed and is no longer available.]

\begin{tabular}{|c|c|c|c|c|c|}
\hline \multicolumn{6}{|c|}{ 2-Meter orange point sampler } \\
\hline Date & Clock time & $\begin{array}{c}\text { Elapsed time } \\
\text { (min) }\end{array}$ & $\begin{array}{l}\text { Ethanol } \\
(\mathrm{mg} \mathrm{C} / \mathrm{L})\end{array}$ & $\begin{array}{l}\text { Benzoate } \\
(\mathrm{mg} \mathrm{C} / \mathrm{L})\end{array}$ & $\begin{array}{l}\mathrm{CCl}_{2} \mathrm{~F}_{2} \\
(\mathrm{ng} / \mathrm{L})\end{array}$ \\
\hline $3-1-78$ & 2130 & 0 & 3 & $*$ & * \\
\hline Do. & 2135 & 5 & $\star$ & 0 & $*$ \\
\hline Do. & 2140 & 10 & $*$ & 0 & $*$ \\
\hline Do. & 2145 & 15 & $*$ & 0 & $*$ \\
\hline Do. & 2150 & 20 & $*$ & 0 & $*$ \\
\hline Do. & 2155 & 25 & $*$ & 0 & $*$ \\
\hline Do. & 2200 & 30 & 5 & .03 & $*$ \\
\hline Do. & 2205 & 35 & 6 & .06 & 3.30 \\
\hline Do. & 2210 & 40 & 7 & .16 & 2.20 \\
\hline Do. & 2215 & 45 & 8 & .5 & 2.51 \\
\hline Do. & 2220 & 50 & 10 & .96 & 5.96 \\
\hline Do. & 2225 & 55 & 10 & 1.61 & 2.04 \\
\hline Do. & 2230 & 60 & 12 & 2.12 & 6.90 \\
\hline Do. & 2235 & 65 & 12 & 2.6 & 6.43 \\
\hline Do. & 2240 & 70 & 13 & 3.1 & $*$ \\
\hline Do. & 2245 & 75 & 13 & 3.47 & $*$ \\
\hline Do. & 2250 & 80 & 13 & 3.66 & $*$ \\
\hline Do. & 2255 & 85 & $*$ & 3.9 & $*$ \\
\hline Do. & 2300 & 90 & 11 & 4.1 & 3.45 \\
\hline Do. & 2310 & 100 & 11 & 3.34 & 11.1 \\
\hline Do. & 2320 & 110 & 11 & 2.51 & $*$ \\
\hline Do. & 2330 & 120 & 12 & 1.41 & 27.0 \\
\hline Do. & 2345 & 135 & 13 & .68 & 12.9 \\
\hline
\end{tabular}


Table 25.--Data obtained from the second tracer experiment on March 1, 1978, at the Stanton, Tex. site--Continued

\begin{tabular}{cccccc}
\hline \multicolumn{7}{c}{ 2-Meter orange point sampler } & \\
\hline Date & Clock time & $\begin{array}{c}\text { Elapsed time } \\
(\mathrm{min})\end{array}$ & $\begin{array}{c}\text { Ethanol } \\
(\mathrm{mg} \mathrm{C} / \mathrm{L})\end{array}$ & $\begin{array}{c}\text { Benzoate } \\
(\mathrm{mg} \mathrm{C} / \mathrm{L})\end{array}$ & $\begin{array}{c}\mathrm{CC1}_{2} \mathrm{~F}_{2} \\
(\mathrm{ng} / \mathrm{L})\end{array}$ \\
\hline 3-2-78 & 2400 & 150 & 12 & .25 & 4.86 \\
Do. & 0015 & 165 & 9 & 0.13 & $*$ \\
Do. & 0030 & 180 & 9 & .06 & $*$ \\
Do. & 0100 & 210 & 9 & .06 & $*$ \\
Do. & 0130 & 240 & 9 & .06 & 4.39 \\
Do. & 0230 & 300 & 6 & 0 & $*$ \\
Do. & 0400 & 390 & 5 & 0 & $*$ \\
Do. & 0600 & 510 & 2 & 0 & $*$ \\
Do. & 0800 & 630 & 1 & 0 & $*$ \\
\hline
\end{tabular}


Table 26.--Data obtained from the second tracer experiment on March 1, 1978, at the Stanton, Tex. site

[Samples are from the point sampler designated 2-m green located $2 \mathrm{~m}$ radially from the injection well and $32.6 \mathrm{~m}$ below land surface. The asterisk $(*)$ indicates the sample was not analyzed and is no longer available.]

\begin{tabular}{|c|c|c|c|c|c|}
\hline \multicolumn{6}{|c|}{ 2-Meter green point sampler } \\
\hline Date & Clock time & $\begin{array}{c}\text { Elapsed time } \\
\text { (min) }\end{array}$ & $\begin{array}{l}\text { Ethanol } \\
(\mathrm{mg} \mathrm{C} / \mathrm{L})\end{array}$ & $\begin{array}{l}\text { Benzoate } \\
(\mathrm{mg} \mathrm{C} / \mathrm{L})\end{array}$ & $\begin{array}{l}\mathrm{CCl}_{2} \mathrm{~F}_{2} \\
(\mathrm{ng} / \mathrm{L})\end{array}$ \\
\hline $3-1-78$ & 2130 & 0 & $*$ & $*$ & $*$ \\
\hline Do. & 2135 & 5 & 4.0 & 0.00 & $*$ \\
\hline Do. & 2140 & 10 & 4.0 & .00 & $*$ \\
\hline Do. & 2220 & 50 & 4.0 & .10 & $*$ \\
\hline Do. & 2225 & 55 & 4.0 & .17 & $*$ \\
\hline Do. & 2230 & 60 & 4.0 & .30 & $*$ \\
\hline Do. & 2235 & 65 & $*$ & .47 & $*$ \\
\hline Do. & 2240 & 70 & $*$ & .53 & $*$ \\
\hline Do. & 2245 & 75 & 7 & .60 & $*$ \\
\hline Do. & 2250 & 80 & 7 & .70 & $*$ \\
\hline Do. & 2255 & 85 & $*$ & .80 & $*$ \\
\hline Do. & 2300 & 90 & 7 & .97 & $*$ \\
\hline Do. & 2310 & 100 & 9 & .97 & $*$ \\
\hline Do. & 2320 & 110 & 9 & 1.17 & $*$ \\
\hline Do. & 2330 & 120 & 10 & 1.27 & $*$ \\
\hline Do. & 2335 & 125 & * & $*$ & $*$ \\
\hline Do. & 2345 & 135 & 9 & 1.20 & $*$ \\
\hline Do. & 2400 & 150 & 9 & 1.20 & $*$ \\
\hline $3-2-78$ & 0015 & 165 & 10 & 1.17 & $*$ \\
\hline Do. & 0030 & 180 & 10 & 1.13 & $*$ \\
\hline Do. & 0100 & 210 & 10 & 1.13 & $*$ \\
\hline Do. & 0130 & 240 & 11 & 1.03 & $*$ \\
\hline Do. & 0230 & 300 & 7 & .83 & $\ddot{*}$ \\
\hline Do. & 0400 & 390 & 6 & .57 & $*$ \\
\hline Do. & 0600 & 510 & 2 & .27 & $*$ \\
\hline Do. & 0800 & 630 & 2 & .13 & $*$ \\
\hline
\end{tabular}


Table 27.--Data obtained from the second tracer experiment on March 1, 1978, at the Stanton, Tex. site

[Samples are from the observation well located $5 \mathrm{~m}$ from the injection well. The 5-m well is screened for full-saturated thickness of the aquifer. The asterisk $(*)$ indicates the sample was not analyzed and is no longer available.]

\begin{tabular}{|c|c|c|c|c|c|}
\hline \multicolumn{6}{|c|}{ 5-Meter well } \\
\hline Date & Clock time & $\begin{array}{c}\text { Elapsed time } \\
\text { (min) }\end{array}$ & $\begin{array}{l}\text { Ethanol } \\
(\mathrm{mg} \mathrm{C} / \mathrm{L})\end{array}$ & $\begin{array}{l}\text { Benzoate } \\
(\mathrm{mg} \mathrm{C} / \mathrm{L})\end{array}$ & $\begin{array}{l}\mathrm{CCl}_{2} \mathrm{~F}_{2} \\
(\mathrm{ng} / \mathrm{L})\end{array}$ \\
\hline $3-1-78$ & 2145 & 15 & 4 & 0 & $\star$ \\
\hline Do. & 2205 & 35 & 4 & 0 & 5.81 \\
\hline Do. & 2235 & 65 & 5 & 0 & $*$ \\
\hline Do. & 2245 & 75 & 4 & .15 & $*$ \\
\hline Do. & 2255 & 85 & $*$ & .23 & $*$ \\
\hline Do. & 2300 & 90 & $*$ & $*$ & $*$ \\
\hline Do. & 2315 & 105 & $*$ & .46 & 5.81 \\
\hline Do. & 2320 & 110 & $*$ & $*$ & $*$ \\
\hline Do. & 2325 & 115 & $*$ & .54 & $*$ \\
\hline Do. & 2340 & 130 & 3 & .77 & $*$ \\
\hline Do. & 2400 & 150 & $*$ & .85 & 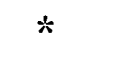 \\
\hline $3-2-78$ & 0010 & 160 & $*$ & $*$ & $*$ \\
\hline Do. & 0015 & 165 & $*$ & 1.0 & 7.85 \\
\hline Do. & 0020 & 170 & $\star$ & $*$ & $*$ \\
\hline Do. & 0030 & 180 & 4 & 1.0 & $*$ \\
\hline Do. & 0100 & 210 & 3 & .92 & $*$ \\
\hline Do. & 0130 & 240 & 4 & .77 & 11.9 \\
\hline Do. & 0230 & 300 & 5 & .62 & 23.9 \\
\hline Do. & 0300 & 330 & 4 & .54 & $*$ \\
\hline Do. & 0400 & 390 & 1 & .31 & 29.9 \\
\hline Do. & 0600 & 510 & $*$ & $*$ & $*$ \\
\hline DO. & 0630 & 540 & $*$ & .15 & 29.9 \\
\hline Do. & 0815 & 645 & $*$ & $*$ & 25.9 \\
\hline Do. & 1100 & 810 & $*$ & $\star$ & 11.9 \\
\hline
\end{tabular}


Table 28.--Data obtained from the second tracer experiment on March 1, 1978, at the Stanton, Tex. site

ISamples are from the point sampler 5-m black located $5 \mathrm{~m}$ radially from the injection well and $41.8 \mathrm{~m}$ below land surface. The asterisk (*) indicates the sample was not analyzed and is no longer available.]

\begin{tabular}{|c|c|c|c|c|c|}
\hline \multicolumn{6}{|c|}{ 5-Meter black point sampler } \\
\hline Date & Clock time & $\begin{array}{c}\text { Elapsed time } \\
\text { (min) }\end{array}$ & $\begin{array}{l}\text { Ethanol } \\
\text { (mg C/L) }\end{array}$ & $\begin{array}{l}\text { Benzoate } \\
\text { (mg } \mathrm{C} / \mathrm{L} \text { ) }\end{array}$ & $\begin{array}{l}\mathrm{CC}_{2}{ }_{2}{ }_{2} \\
\text { (ng/L) }\end{array}$ \\
\hline $3-1-78$ & 2140 & 10 & $*$ & 0 & $*$ \\
\hline Do. & 2210 & 40 & * & 0 & $*$ \\
\hline Do. & 2230 & 60 & 4 & $*$ & $*$ \\
\hline Do. & 2240 & 70 & $*$ & 0 & $*$ \\
\hline Do. & 2330 & 120 & 4 & $*$ & $*$ \\
\hline Do. & 2345 & 135 & $*$ & .01 & $*$ \\
\hline Do. & 2400 & 150 & 5 & $*$ & $*$ \\
\hline $3-2-78$ & 0030 & 180 & 4.5 & .13 & $*$ \\
\hline Do. & 0100 & 210 & $*$ & .30 & $*$ \\
\hline Do. & 0130 & 240 & 6 & .33 & $*$ \\
\hline Do. & 0200 & 270 & 7 & .33 & $*$ \\
\hline Do. & 0230 & 300 & 5.5 & .33 & $*$ \\
\hline Do. & 0400 & 390 & 5 & .37 & $\star$ \\
\hline Do. & 0600 & 510 & 6 & .33 & $*$ \\
\hline Do. & 0800 & 630 & $*$ & .27 & $*$ \\
\hline Do. & 0945 & 735 & $*$ & .20 & $*$ \\
\hline
\end{tabular}


Table 29.--Data obtained from the second tracer experiment on March 1, 1978, at the Stanton, Tex. site

[Samples are from the point sampler designated 5-m orange located $37.5 \mathrm{~m}$ below land surface and $5 \mathrm{~m}$ radially from the injection well. The asterisk $(*)$ indicates the sample was not analyzed and is no longer available.]

\begin{tabular}{|c|c|c|c|c|c|}
\hline \multicolumn{6}{|c|}{ 5-Meter orange point sampler } \\
\hline Date & Clock time & $\begin{array}{c}\text { Elapsed time } \\
(\min )\end{array}$ & $\begin{array}{l}\text { Ethanol } \\
(\mathrm{mg} \mathrm{C} / \mathrm{L})\end{array}$ & $\begin{array}{l}\text { Benzoate } \\
(\mathrm{mg} \mathrm{C} / \mathrm{L})\end{array}$ & $\begin{array}{l}\mathrm{CC}_{2} \mathrm{~F}_{2} \\
(\mathrm{ng} / \mathrm{L})\end{array}$ \\
\hline $3-1-78$ & 2140 & 0 & 1 & 0 & 0 \\
\hline Do. & 2200 & 20 & $*$ & $*$ & $*$ \\
\hline Do. & 2210 & 30 & 4 & 0 & $*$ \\
\hline Do. & 2220 & 40 & 4 & 0 & $*$ \\
\hline Do. & 2230 & 50 & 3 & .07 & 3.77 \\
\hline Do. & 2240 & 60 & 4 & $*$ & $*$ \\
\hline Do. & 2250 & 70 & 4 & .13 & 16.2 \\
\hline Do. & 2300 & 80 & 4 & .20 & 16.2 \\
\hline Do. & 2310 & 90 & $*$ & .23 & 21.7 \\
\hline Do. & 2320 & 100 & 4 & .30 & 28.9 \\
\hline Do. & 2330 & 110 & 6 & .33 & $*$ \\
\hline Do. & 2345 & 125 & 5 & .43 & $*$ \\
\hline Do. & 2400 & 140 & 5 & .53 & $*$ \\
\hline $3-2-78$ & 0015 & 155 & 6 & .80 & $*$ \\
\hline Do. & 0030 & 170 & 6 & .87 & 25.9 \\
\hline Do. & 0100 & 200 & $*$ & .80 & $*$ \\
\hline Do. & 0130 & 230 & 7 & .63 & 17.9 \\
\hline Do. & 0200 & 260 & 8 & .53 & 34.5 \\
\hline Do. & 0230 & 290 & 7 & .40 & $*$ \\
\hline Do. & 0400 & 380 & 5 & .33 & $*$ \\
\hline Do. & 0600 & 500 & 5 & .33 & $*$ \\
\hline Do. & 0800 & 620 & 4 & .30 & $*$ \\
\hline Do. & 0945 & 725 & 1 & 0 & $*$ \\
\hline
\end{tabular}


Because of the positive indications of well-bore plugging during the March injection test, the injection well was redeveloped during April 1978. A step-drawdown test was performed prior to development, which indicated that the maximum yield of the well was $4.7 \mathrm{~L} / \mathrm{s}$ with $9.15 \mathrm{~m}$ of drawdown. The well was then acidized with $110 \mathrm{~kg}$ of sulfamic acid and bailed. Following bailing, an inflatable packer was set in the injection well at a depth of $39.6 \mathrm{~m}$, and the well was swabbed through a 102-mm diameter swab pipe extending below the packer. The packer prevented water from being swabbed from well-bore storage, and allowed the aquifer to be stressed during the swabbing operation.

Following the acid treatment, bailing, and swabbing operations, the well was pumped again to determine its maximum sustainable yield. The well was pumped at a rate of $13.9 \mathrm{~L} / \mathrm{s}$ with a drawdown of $8.5 \mathrm{~m}$ after about $4 \mathrm{~h}$ of pumping; this was the capacity of the pump. These data indicate a more than three-fold improvement in the injection-well yield.

\section{MAY 1978 INJECTION TEST}

The main experimental objective of the Artificial-Recharge Research project for the Stanton site was to monitor and model the quality of injected playa-lake water. However, achievement of this goal had been continually thwarted by the weather. Runoff accumulations in the playa lake were minimal during the spring, summer, and fall of 1977, when the tests were initially scheduled, and the lake was dry during most of that time. The lowest inlet for the lake-lift pump, which had become blocked by silt, was cleaned out during the spring of 1978. Enough runoff collected in the lake from a rainstorm on May 22, 1978 to allow a 3-d injection test at an intended injection rate of $10 \mathrm{~L} / \mathrm{s}$. A test of this duration and rate was considered the minimum that would meet the project objectives. Although it had not been possible to maintain that large an injection rate during the March test, it was believed that the development work completed during April that resulted in a production rate of $13.7 \mathrm{~L} / \mathrm{s}$ was sufficient to allow the $10-\mathrm{L} / \mathrm{s}$ injection rate.

For the test, which started on May 24, 1979, water was picked up by the lake-lift pump, and a flocculating agent (Nalco 607) was added at a rate of about $10 \mathrm{mg} / \mathrm{L}$ at the pump. Water was routed through the horseshoe settling basin and was picked up by the lift pump in the building, with diatomaceous earth added as a filter aid. Water was routed through one of the diatomaceousearth filters, and various tracers were added downstream from the filter.

The injection sequence was similar to that for the March test, with tracer concentrations being adjusted to the desired amounts while the injection stream was run to waste. Injection of the tracer-bearing water was then started using the quick-connect procedure. 
Injection was started at a rate of slightly less than $10 \mathrm{~L} / \mathrm{s}$ at 1400 on May 24. The well plugged even more rapidly than it had during the March test, and the injection rate had to be continually decreased. Injection was temporarily halted at 2220 on May 24 because of uncertainties resulting from the clogging, and was resumed at 1101 on May 25. After about 6 hours of injection at a continually decreasing rate, the test was stopped. Flow rates and cumulative volumes of injected water after selected times are listed in table 30 .

Post-test experiments indicated that the filter tanks and recharge line were not adequately purged of air before injection started. Because the plant had originally been designed to recharge much larger quantities of water than could actually be injected, output from the plant had to be severely restricted by valves. Air apparently became entrained in the injection stream, both from the air reservoir remaining in the tanks and line, and from a Venturi effect immediately downstream from one or more of the nearly-closed valves. Injection of the aerated water resulted in the severe plugging observed during the test.

\section{Hydraulic Data}

Hydraulic data for the May test include, in addition to the injection rates, periodic vertical flow-meter logs made in the injection well (fig. 13). These logs show the zones that were taking water at selected intervals during the test.

Water levels were closely monitored in the various piezometers and in selected observation wells during the May test. However, water-level data are not readily interpretable by standard methods because of the extreme variability in the injection rate during the test; these data are not included.

\section{Chemical and Tracer Data}

Despite the problems involving clogging during injection, data collected during the test provide valuable comparisons between the dissolved organic material naturally present in the playa water as a natural tracer and the added tracers. The playa-lake water and the ground water had significantly different compositions (table 31); consequently, the alkalinity, specific conductance, and major-cation chemistry were monitored as well. The cation-exchange process was only beginning to have a detectable effect on water composition at the termination of the test; those data are not presented. Specific-conductance and alkalinity data are presented in figures 14 to 17 .

Tracers chosen for this test and their concentrations are shown in table 32. A new analytical method for bromide was used; this proved unsatisfactory, so only boron, fluorocarbon, and natural-organics data are presented. 


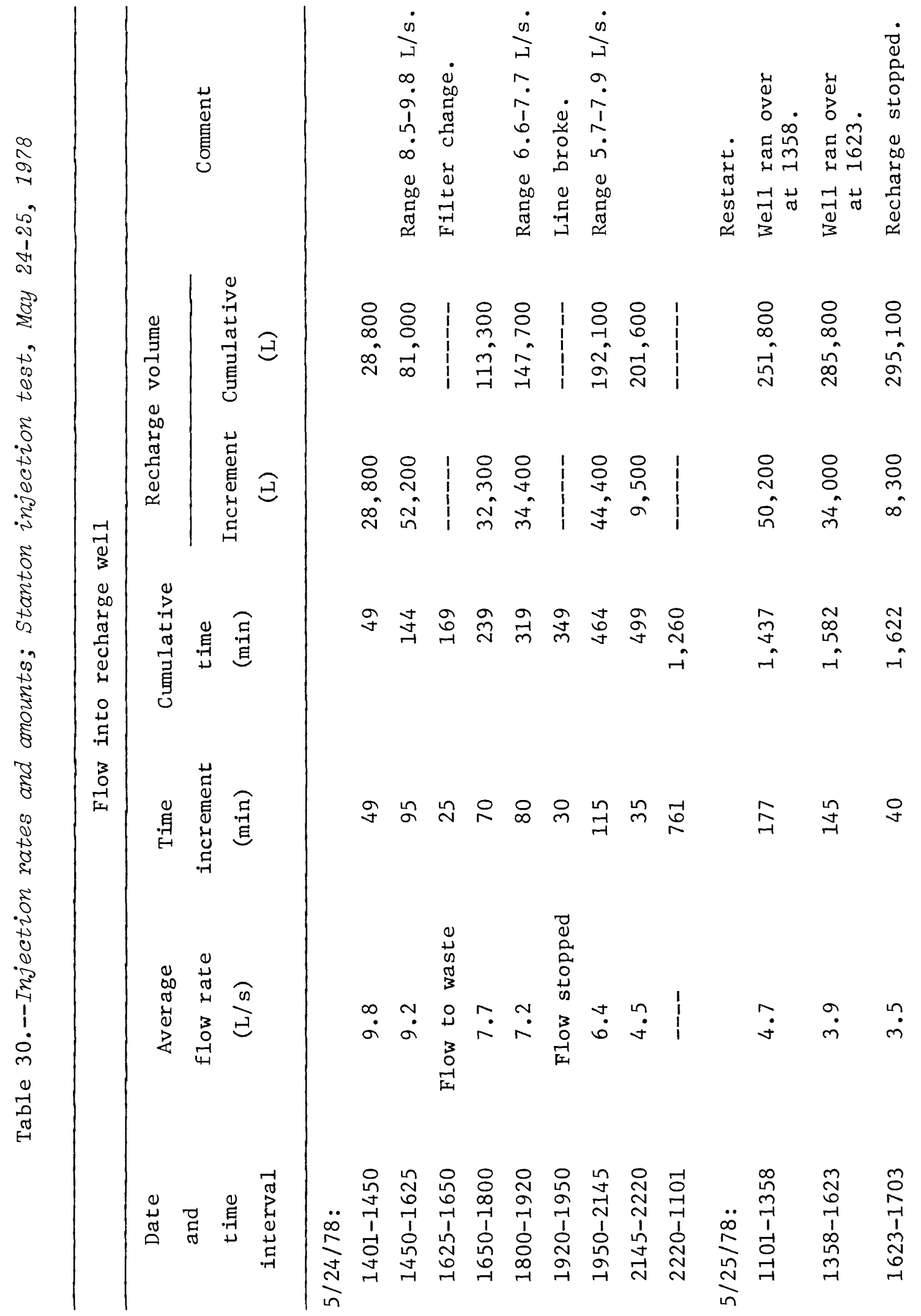




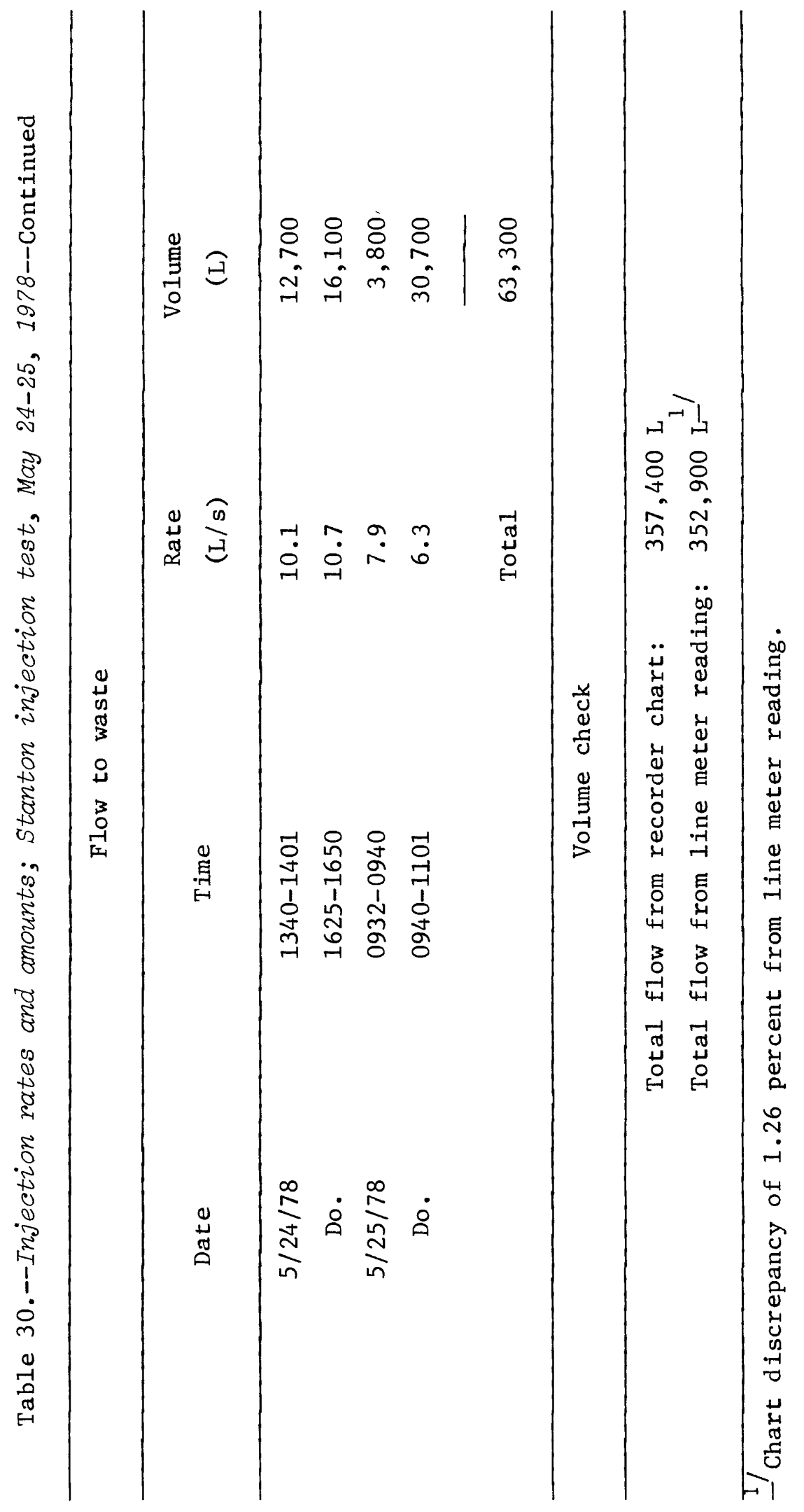



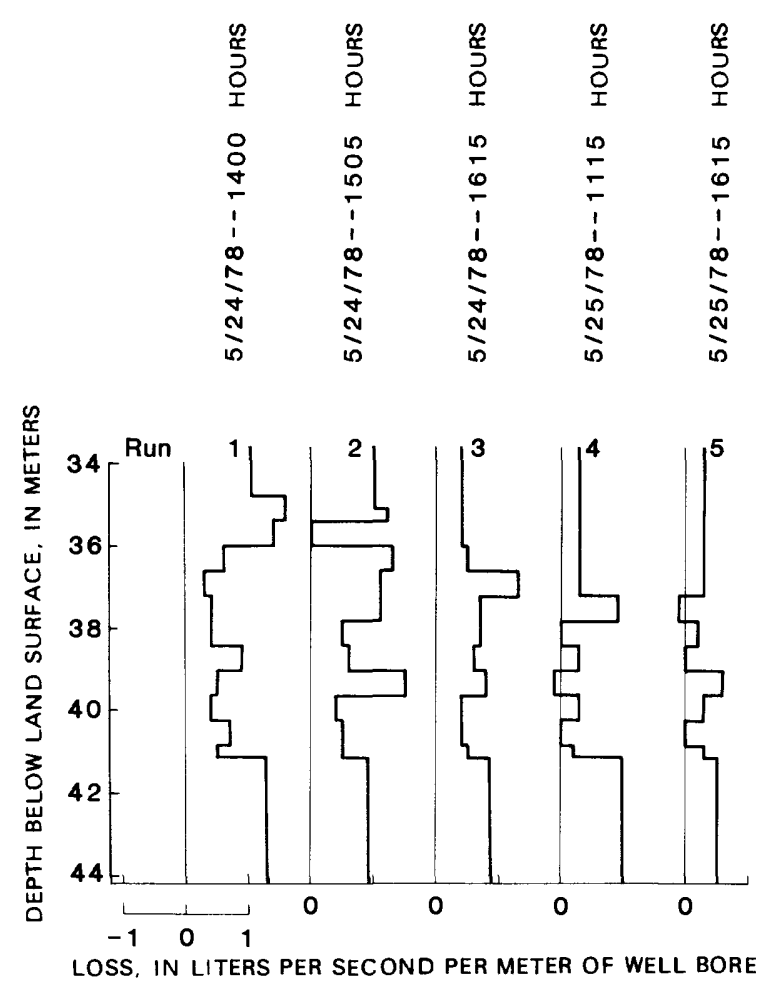

Figure 13.--Flowmeter logs from the injection well obtained during the May 1978 test. 
Table 31.--Analyses of the playa-lake water and ground water prior to the May 24-25, 1978, injection test

\begin{tabular}{|c|c|c|}
\hline Constituent & $\begin{array}{c}\text { Playa lake } \\
(\mathrm{mg} / \mathrm{L})\end{array}$ & $\begin{array}{c}\text { Ground water } \\
(\mathrm{mg} / \mathrm{L})\end{array}$ \\
\hline Calcium ( $\mathrm{Ca})-$ & 27 & 37 \\
\hline Magnesium (Mg) - & 2.5 & 23 \\
\hline Sodium (Na)- & 7.7 & 8.4 \\
\hline Potassium $(\mathrm{K})-$ & 6.0 & 20 \\
\hline Chloride (C1) & 12 & 19 \\
\hline Sulfate $\left(\mathrm{SO}_{4}\right)$ & 14 & 28 \\
\hline On-site alkalinity $\left(\mathrm{HCO}_{3}\right)$ & 31 & 116 \\
\hline Laboratory alkalinity $\left(\mathrm{CaCO}_{3}\right)$ & 60 & 170 \\
\hline Lithium (Li) - & $<.005$ & .06 \\
\hline Nitrate $(\mathrm{N})$ & .31 & .6 \\
\hline Silica $\left(\mathrm{SiO}_{2}\right)$ & 5.9 & 44 \\
\hline Strontium (Sr) - & .210 & 1.4 \\
\hline Boron (B)-- & .23 & .110 \\
\hline Iron $(\mathrm{Fe})-$ & .04 & $<.005$ \\
\hline Fluoride (F) & .3 & 1.9 \\
\hline Bromide (Br) & .5 & .1 \\
\hline Manganese (Mn)-- & .02 & .47 \\
\hline pH on-site (units) - & 7.84 & 7.95 \\
\hline Specific conductance $(\mu \mathrm{mhos} / \mathrm{cm})-$ & 203 & 462 \\
\hline Dissolved solids- & 115 & 288 \\
\hline
\end{tabular}




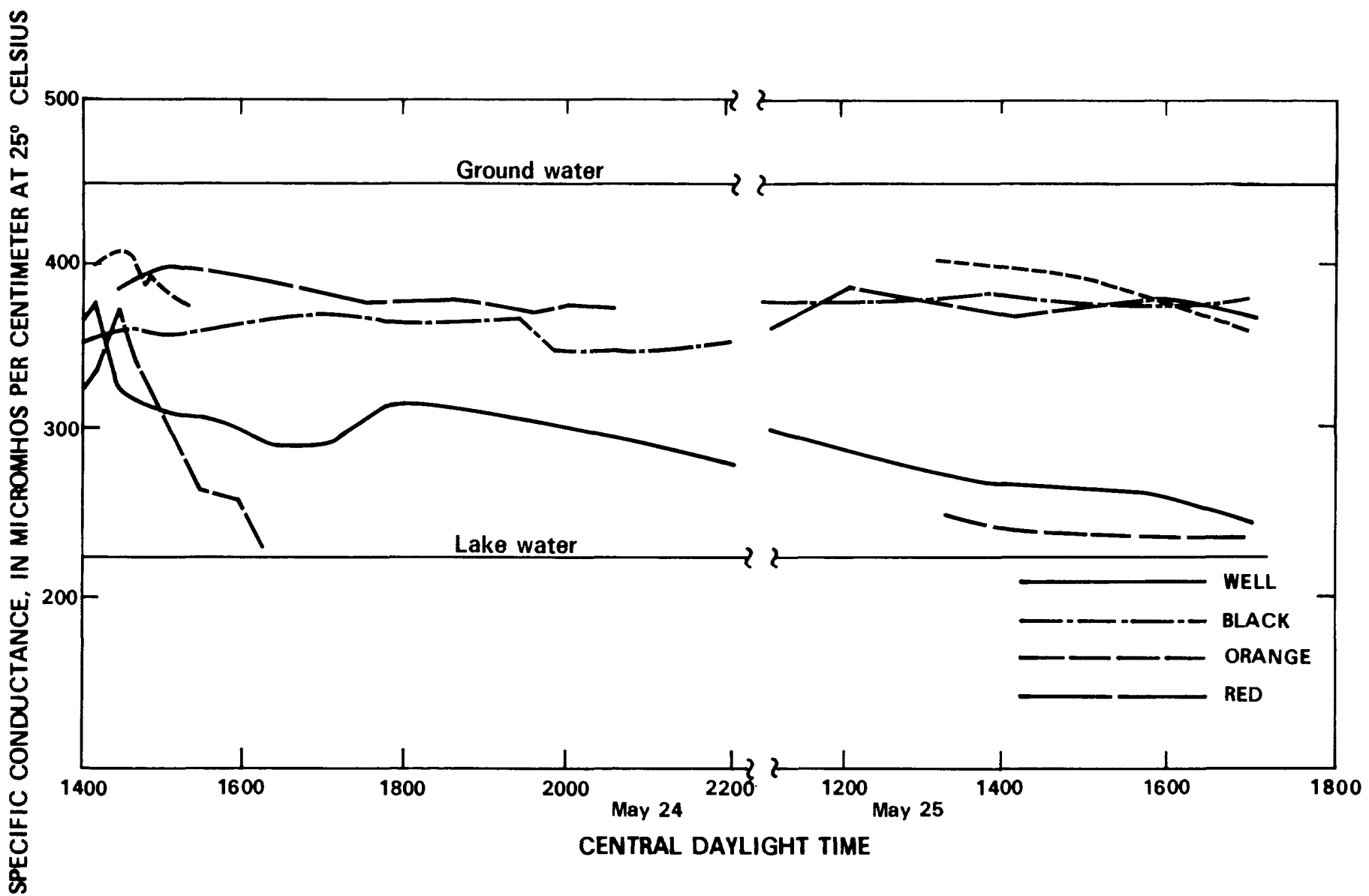

Figure 14.--Change in specific conductance measured in samples from the 2-meter well and point samplers collected May 24-25, 1978. 


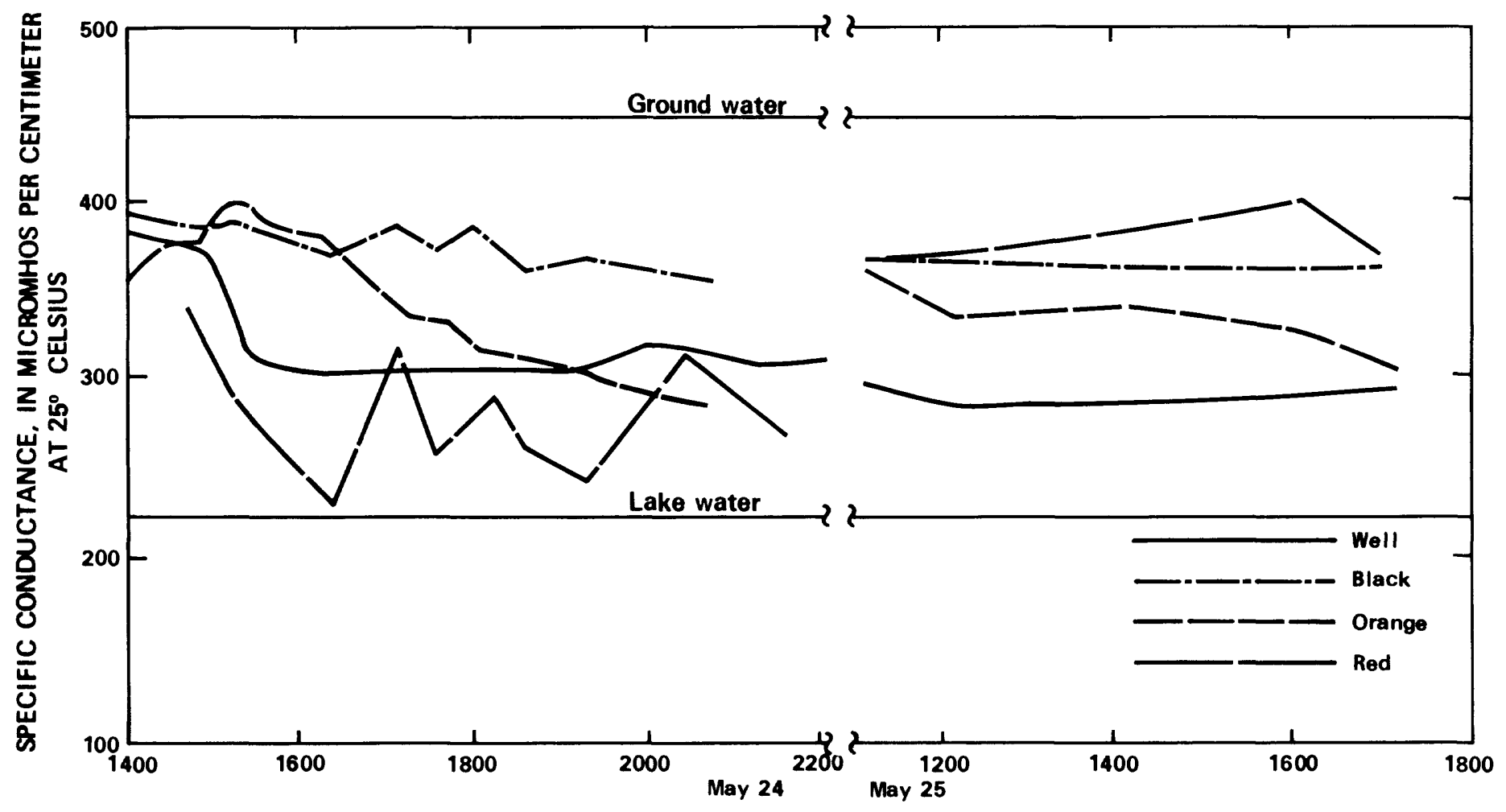

CENTRAL DAYLIGHT TIME

Figure 15.--Change in specific conductance measured in samples from the 5-meter well and point samplers collected May 24-25, 1978. 


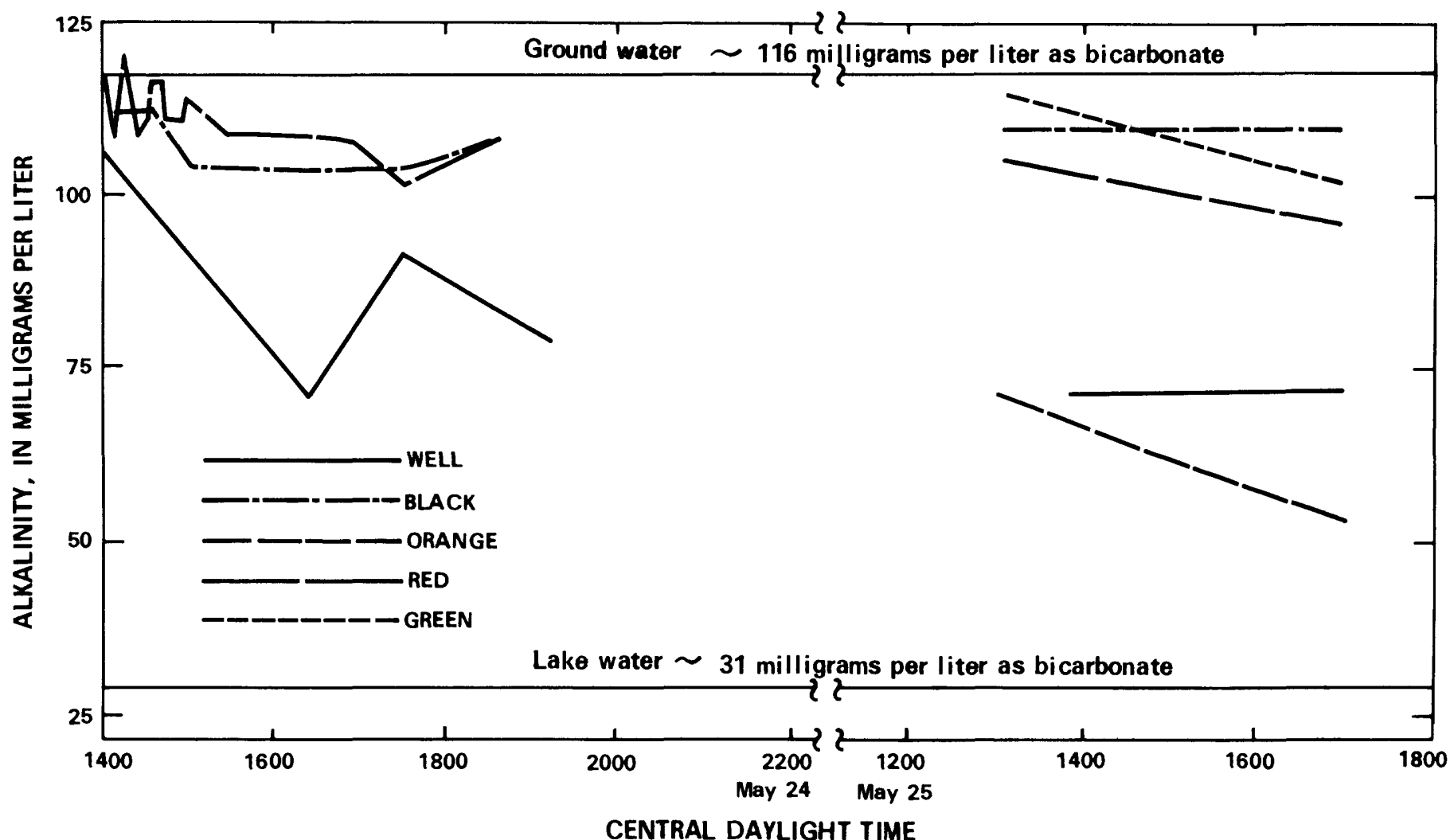

Figure 16.--Change in alkalinity measured in samples from the 2-meter well. and point samplers collected May 24-25, 1978. 


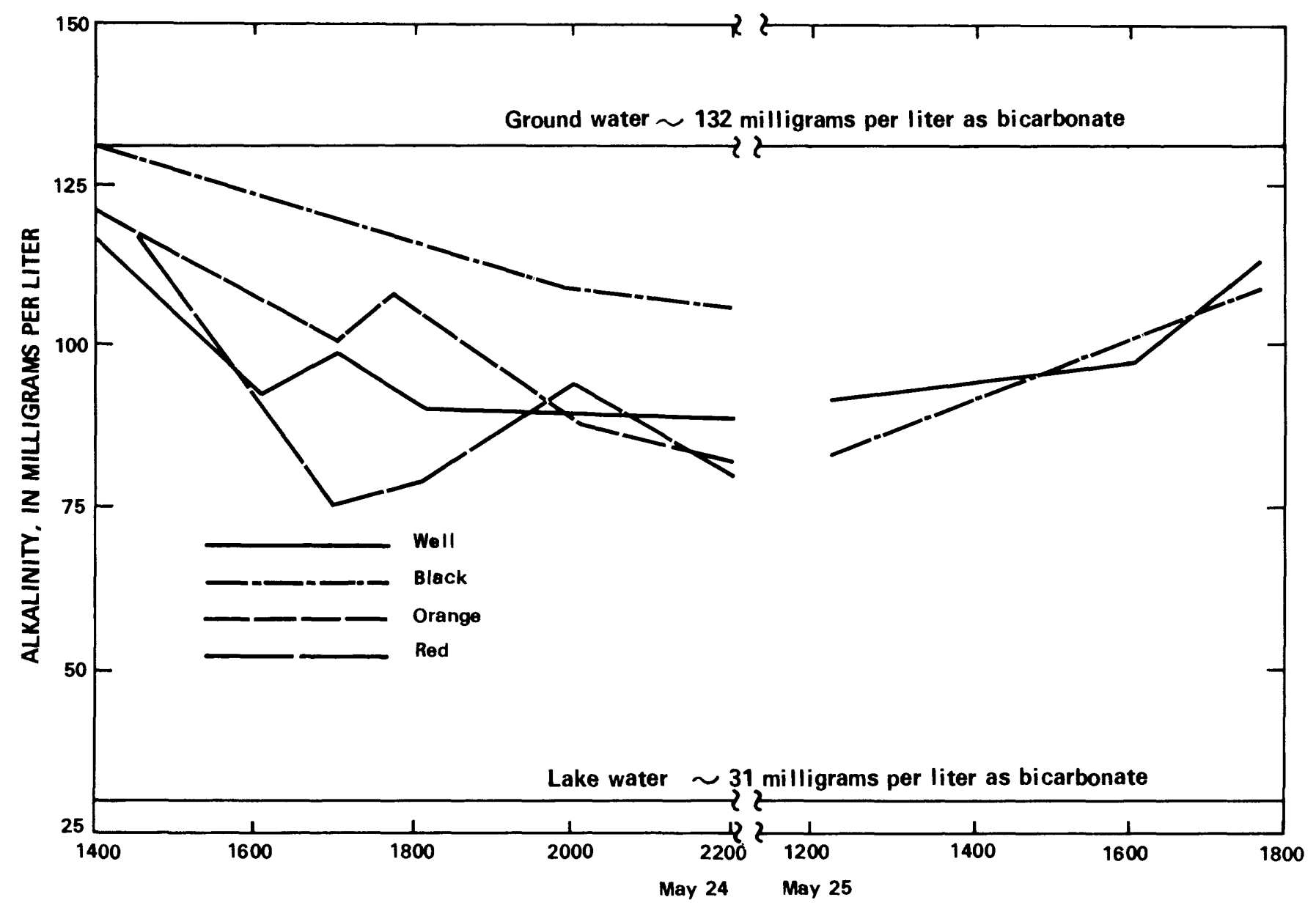

CENTRAL DAYLIGHT TIME

Figure 17.--Change in alkalinity measured in samples from the 5-meter well and point samplers collected May 24-25, 1978. 
Table 32.--Tracer information for May injection

\begin{tabular}{|c|c|c|c|c|}
\hline Tracer & $\begin{array}{l}\text { Length of } \\
\text { injection }\end{array}$ & Start time & Date & $\begin{array}{c}\text { Initial } \\
\text { concentration }\end{array}$ \\
\hline Boron----_--_-_-_-- & Continuous & 1400 & $5 / 24 / 78$ & $982 \mu \mathrm{g} / \mathrm{L}$ \\
\hline Bromide--_-_-_-_--- & do. & 1400 & do. & $1.20 \mathrm{mg} / \mathrm{L}$ \\
\hline \multicolumn{5}{|l|}{ Natural playa- } \\
\hline dissolved organics-- & do. & 1400 & do. & $8.4 \mathrm{mg} \mathrm{C} / \mathrm{L}$ \\
\hline $\mathrm{CCl}_{2} \mathrm{~F}_{2}$ & do. & 1400 & do. & $30 \mu \mathrm{g} / \mathrm{L}$ \\
\hline $\mathrm{CBrClF}_{2}-$ & do. & 1400 & do. & $22 \mu \mathrm{g} / \mathrm{L}$ \\
\hline $\mathrm{CBr}_{2} \mathrm{~F}_{2}-\cdots$ & do. & 1400 & do. & $28 \mu \mathrm{g} / \mathrm{L}$ \\
\hline
\end{tabular}


Sampling Schedule and Tracer Data

The injection test began at 1400 on May 24, 1978; sampling of ground water and monitoring of water levels began at that time. Virtually the same sampling frequency used during the March test was used during this experiment. These samples were analyzed to determine the tracer concentrations at selected times, as listed in tables 33 through 43. Data shown in these tables are labeled with the same location designations used for the data obtained during the March test (tables 22 through 29). The fluorocarbon data are presented as the unitless ratio $\mathrm{C} / \mathrm{Co}$, because problems with the injection pump caused an irregular delivery of the tracer into the injection stream. Data are presented as the ratio to input concentration in order to compensate for this difficulty.

\section{AUGUST 1978 INJECTION TEST}

The August injection test was run using ground water to provide a final evaluation of various ground-water tracers. Prior to the test, the injection wel1 had again been acidized and swabbed, as it had been during April. A subsequent pump test indicated that the well had a sustainable yield of $13.2 \mathrm{~L} / \mathrm{s}$, somewhat less than the $13.7 \mathrm{~L} / \mathrm{s}$ yield measured during April, but substantially greater than its yield of $7.6 \mathrm{~L} / \mathrm{s}$ measured after the May injection test, but before redevelopment.

Water for the test was obtained from wells 2 and 3 (fig. 1), and was routed through the treatment plant by bypassing the diatomaceous-earth filters. We11s 2 and 3 were pumped at rates of about 1.26 and $3.79 \mathrm{~L} / \mathrm{s}$, respectively, for a combined rate of $5.05 \mathrm{~L} / \mathrm{s}$. Ground-water injection was started at 1754 on August 5, 1979, about $3 \mathrm{~d}$ before any chemical tracers were added, to stabilize the flow pattern and minimize the effect on tracer transit time resulting from ground-water storage near the injection well. These initial conditions for the start of tracer injection differ from those for the March and May tests, when water and tracer injection were started simultaneously. These differences need to be considered when results of the different tests are compared.

\section{Hydraulic Data}

Submersible pumps equipped with 7.5 and $3.75-\mathrm{kW}$ electric motors were used to pump wells 3 and 2, respectively. Because these motors were much smaller than the 38-kW motor used to pick up water from the horseshoesettling basin, valves in the system were much less constricted, minimizing the chance for air to be entrained by a Venturi effect. Consequently, plugging was minimal; water level in the injection well was relatively stable; and the injection rate could be held constant at $5.05 \mathrm{~L} / \mathrm{s}$ within the accuracy of the flow meters throughout the test. Cumulative volumes of injected water at the start of each tracer injection are listed in table 44. 
Table 33.--Data obtained from water samples collected during the May 24-25, 1978, injection test using playa-lake water

[Samples are from the observation well located $2 \mathrm{~m}$ from the injection well.

The 2-m well is screened for the full-saturated thickness of the aquifer.

The dash (-) indicates sample is available but was not analyzed; the asterisk (*) indicates the sample was not analyzed and is no longer available.]

\begin{tabular}{|c|c|c|c|c|c|c|c|}
\hline \multicolumn{8}{|c|}{ 2-Meter well } \\
\hline \multirow{2}{*}{ Date } & \multirow{2}{*}{ Clock time } & \multirow{2}{*}{$\begin{array}{c}\text { Elapsed } \\
\text { time } \\
\text { (min) }\end{array}$} & \multirow{2}{*}{$\begin{array}{l}\text { Boron } \\
(\mu g / L)\end{array}$} & \multirow{2}{*}{$\begin{array}{l}\text { Natural } \\
\text { organics } \\
(\mathrm{mg} \mathrm{C} / \mathrm{L})\end{array}$} & \multicolumn{3}{|c|}{$\mathrm{C} / \mathrm{Co} \times 10^{5}$} \\
\hline & & & & & $\mathrm{F}-12$ & $\mathrm{BCF}$ & DBM \\
\hline $5-24-78$ & 1401 & 1 & 80 & --- & 7.0 & 1.4 & 1.2 \\
\hline Do. & 1403 & 3 & ---- & 4 & $*$ & $*$ & $*$ \\
\hline Do. & 1405 & 5 & ---- & 2 & $*$ & $*$ & $*$ \\
\hline Do. & 1406 & 6 & ----- & .8 & 6.5 & 1.4 & 1.0 \\
\hline Do. & 1410 & 10 & ---- & 1.1 & 8.0 & 1.4 & $*$ \\
\hline Do. & 1417 & 17 & --- & .6 & 400 & 17 & 15 \\
\hline Do. & 1422 & 22 & --- & 3 & 130 & 450 & 680 \\
\hline Do. & 1426 & 26 & ---- & 3.3 & 170 & 800 & 760 \\
\hline Do. & 1431 & 31 & ---- & 3.3 & 84 & 250 & 560 \\
\hline Do. & 1435 & 35 & ---- & 3.2 & 84 & 120 & 340 \\
\hline Do. & 1441 & 41 & ---- & 3.2 & 150 & 280 & 740 \\
\hline Do. & 1445 & 45 & ---- & --- & 170 & 230 & 840 \\
\hline Do. & 1451 & 51 & ---- & 3.0 & 170 & 450 & 860 \\
\hline Do. & 1501 & 61 & 540 & 3.2 & $*$ & $*$ & $*$ \\
\hline Do. & 1507 & 67 & ---- & 3.2 & $*$ & $*$ & $*$ \\
\hline Do. & 1515 & 75 & ---- & 3.4 & $*$ & $*$ & $*$ \\
\hline Do. & 1521 & 81 & ---- & 3.5 & $*$ & $*$ & $*$ \\
\hline Do. & 1536 & 96 & --- & 3.6 & $*$ & $*$ & $*$ \\
\hline Do. & 1543 & 103 & --- & 3.8 & $*$ & $*$ & $*$ \\
\hline Do. & 1551 & 111 & ---- & 3.8 & $*$ & $*$ & $*$ \\
\hline Do. & 1556 & 116 & ---- & 4.1 & $*$ & $*$ & $*$ \\
\hline Do. & 1600 & 120 & ----- & 4.2 & $*$ & $*$ & $*$ \\
\hline Do. & 1603 & 123 & 660 & 4.1 & $*$ & $*$ & $*$ \\
\hline Do. & 1611 & 131 & ---- & 3.7 & $*$ & $*$ & $*$ \\
\hline
\end{tabular}


Table 33.--Data obtained from water samples collected during the May 24-25, 1978, injection test using playa-lake water--Continued

\begin{tabular}{|c|c|c|c|c|c|c|c|}
\hline \multicolumn{8}{|c|}{ 2-Meter we11 } \\
\hline \multirow{2}{*}{ Date } & \multirow{2}{*}{ Clock time } & \multirow{2}{*}{$\begin{array}{l}\text { Elapsed } \\
\text { time } \\
\text { (min) }\end{array}$} & \multirow{2}{*}{$\begin{array}{l}\text { Boron } \\
(\mu g / L)\end{array}$} & \multirow{2}{*}{$\begin{array}{l}\text { Natural } \\
\text { organics } \\
(\mathrm{mg} \mathrm{C} / \mathrm{L})\end{array}$} & \multicolumn{3}{|c|}{$\mathrm{C} / \mathrm{Co} \times 10^{5}$} \\
\hline & & & & & $\mathrm{F}-12$ & $\mathrm{BCF}$ & DBM \\
\hline $5-24-78$ & 1620 & 140 & $-\cdots-$ & 3.9 & 1,820 & 2,200 & 1,900 \\
\hline Do. & 1642 & 162 & --- & 3.7 & $*$ & $*$ & $*$ \\
\hline Do. & 1645 & 165 & $-\cdots$ & 3.2 & $*$ & $*$ & $*$ \\
\hline Do. & 1700 & 180 & 650 & 4 & $*$ & $*$ & $*$ \\
\hline Do. & 1714 & 194 & --- & 4.2 & 4,500 & 4,800 & 2,500 \\
\hline Do. & 1726 & 206 & ---- & 3.6 & $*$ & $*$ & $*$ \\
\hline Do. & 1743 & 223 & ---- & 3.6 & $*$ & $*$ & $*$ \\
\hline Do. & 1803 & 243 & 630 & 3.6 & $*$ & $*$ & $*$ \\
\hline Do. & 1815 & 255 & ---- & 3.8 & $*$ & $*$ & $*$ \\
\hline Do. & 1830 & 270 & $-\cdots$ & 3.8 & $*$ & $*$ & $*$ \\
\hline Do. & 1852 & 292 & 670 & 3.5 & 3,200 & 3,800 & 1,200 \\
\hline Do. & 1938 & 338 & ---- & 4.0 & $*$ & $\star$ & $*$ \\
\hline Do. & 2001 & 361 & 720 & 3.6 & $*$ & $*$ & $*$ \\
\hline Do. & 2030 & 390 & ----- & 3.8 & $*$ & $*$ & $*$ \\
\hline Do. & 2205 & 485 & 1,012 & 3.9 & 1,050 & 4,700 & 1,400 \\
\hline Do. & 1105 & 1,265 & 850 & 3.3 & 4,600 & 4,400 & 1,500 \\
\hline Do. & 1200 & 1,320 & -- -- & 3.8 & $*$ & $*$ & $*$ \\
\hline Do. & 1345 & 1,425 & 880 & 4.1 & $*$ & $*$ & $*$ \\
\hline Do. & 1400 & 1,440 & --- & 4.2 & $*$ & $*$ & $*$ \\
\hline Do. & 1556 & 1,556 & 920 & 4.0 & $*$ & $*$ & $*$ \\
\hline Do. & 1700 & 1,620 & 1,000 & 4.0 & $*$ & $*$ & $*$ \\
\hline
\end{tabular}


Table 34.--Data obtained from water samples collected during the May 24-25, 1978, injection test using playa-lake water

[Samples are from the point sampler designated 2-m black located $41.6 \mathrm{~m}$ below land surface and $2 \mathrm{~m}$ radially from the injection well. The dash (-) indicates sample is available but was not analyzed; an asterisk (*) indicates the sample was not analyzed and is no longer available.]

\begin{tabular}{|c|c|c|c|c|c|c|c|}
\hline \multirow{3}{*}{ Date } & \multirow{3}{*}{ Clock time } & \multicolumn{4}{|c|}{ 2-Meter black point sampler } & & \\
\hline & & \multirow{2}{*}{$\begin{array}{l}\text { Elapsed } \\
\text { time } \\
\text { (min) }\end{array}$} & \multirow{2}{*}{$\begin{array}{l}\text { Boron } \\
(\mu \mathrm{g} / \mathrm{L})\end{array}$} & \multirow{2}{*}{$\begin{array}{l}\text { Natural } \\
\text { organics } \\
(\mathrm{mg} \mathrm{C} / \mathrm{L})\end{array}$} & \multicolumn{3}{|c|}{$\mathrm{C} / \mathrm{Co} \times 10^{5}$} \\
\hline & & & & & $\mathrm{F}-12$ & $\mathrm{BCF}$ & DBM \\
\hline $5-24-78$ & 1405 & 5 & -- & 0.6 & $*$ & $*$ & $*$ \\
\hline Do. & 1415 & 15 & --- & .5 & $*$ & $*$ & $\star$ \\
\hline Do. & 1420 & 20 & --- & .7 & $*$ & $*$ & $*$ \\
\hline Do. & 1425 & 25 & -- & .6 & $*$ & $*$ & $*$ \\
\hline Do. & 1430 & 30 & -- & .5 & $*$ & $*$ & $*$ \\
\hline Do. & 1435 & 35 & --- & .5 & $*$ & $*$ & $*$ \\
\hline Do. & 1440 & 40 & --- & .5 & $*$ & $*$ & $*$ \\
\hline Do. & 1445 & 45 & -- & .6 & $*$ & $*$ & $*$ \\
\hline Do. & 1450 & 50 & -- & .6 & $*$ & $*$ & $*$ \\
\hline Do. & 1455 & 55 & 80 & .6 & $*$ & $*$ & $*$ \\
\hline Do. & 1500 & 60 & -- & .6 & $*$ & $*$ & $*$ \\
\hline Do. & 1505 & 65 & -- & .8 & $*$ & $*$ & $*$ \\
\hline Do. & 1510 & 70 & -- & .7 & $*$ & $*$ & $*$ \\
\hline Do. & 1515 & 75 & --- & .6 & $*$ & $*$ & $*$ \\
\hline Do. & 1520 & 80 & 78 & -- & $*$ & $*$ & $*$ \\
\hline Do. & 1530 & 90 & -- & .6 & $*$ & $*$ & $*$ \\
\hline Do. & 1540 & 100 & --- & .7 & $*$ & $*$ & $\star$ \\
\hline Do. & 1550 & 110 & -- & .7 & $*$ & $*$ & $*$ \\
\hline Do. & 1600 & 120 & -- & .8 & $*$ & $*$ & $*$ \\
\hline Do. & 1610 & 130 & --- & .8 & $*$ & $*$ & $*$ \\
\hline Do. & 1620 & 140 & --- & .8 & $*$ & $*$ & $*$ \\
\hline Do. & 1655 & 175 & 80 & .7 & $*$ & $*$ & $*$ \\
\hline Do. & 1700 & 180 & --- & .9 & $*$ & $*$ & $*$ \\
\hline Do. & 1710 & 190 & --- & .6 & $*$ & $*$ & $*$ \\
\hline Do. & 1720 & 200 & --- & 1.2 & $*$ & $*$ & $*$ \\
\hline
\end{tabular}


Table 34.--Data obtained from water samples collected during the May 24-25, 1978, injection test using playa-lake water--Continued

\begin{tabular}{|c|c|c|c|c|c|c|c|}
\hline \multicolumn{8}{|c|}{ 2-Meter black point sampler } \\
\hline \multirow{2}{*}{ Date } & \multirow{2}{*}{ Clock time } & \multirow{2}{*}{$\begin{array}{c}\text { Elapsed } \\
\text { time } \\
\text { (min) }\end{array}$} & \multirow{2}{*}{$\begin{array}{l}\text { Boron } \\
(\mu \mathrm{g} / \mathrm{L})\end{array}$} & \multirow{2}{*}{$\begin{array}{l}\text { Natural } \\
\text { organics } \\
(\mathrm{mg} \mathrm{C} / \mathrm{L})\end{array}$} & \multicolumn{3}{|c|}{$\mathrm{C} / \mathrm{Co} \times 10^{5}$} \\
\hline & & & & & $F-12$ & $\mathrm{BCF}$ & DBM \\
\hline $5-24-78$ & 1730 & 210 & -- & 0.6 & $*$ & $*$ & $*$ \\
\hline Do. & 1740 & 220 & -- & .6 & $*$ & $*$ & $*$ \\
\hline Do. & 1800 & 240 & --- & .7 & $*$ & $*$ & $*$ \\
\hline Do. & 1815 & 255 & 80 & -- & $*$ & $*$ & $*$ \\
\hline Do. & 1830 & 270 & --- & .8 & $*$ & $*$ & $*$ \\
\hline Do. & 1845 & 285 & 90 & .6 & $*$ & $*$ & $*$ \\
\hline Do. & 1900 & 300 & -- & 1.1 & $*$ & $*$ & $*$ \\
\hline Do. & 1920 & 320 & --- & .7 & $*$ & $*$ & $*$ \\
\hline Do. & 1940 & 340 & --- & .9 & $*$ & $*$ & $*$ \\
\hline Do. & 2000 & 360 & -- & .6 & $*$ & $*$ & $*$ \\
\hline Do. & 2030 & 390 & -- & .6 & $*$ & $*$ & $*$ \\
\hline Do. & 2100 & 420 & 80 & .8 & $*$ & $*$ & $*$ \\
\hline Do. & 2200 & 480 & 80 & .8 & $*$ & $*$ & $*$ \\
\hline $5-25-78$ & 1100 & 1,260 & 90 & 1.5 & $*$ & $*$ & $*$ \\
\hline Do. & 1200 & 1,320 & -- & 1.1 & $*$ & $*$ & $*$ \\
\hline Do. & 1300 & 1,380 & 100 & -- & $*$ & $*$ & $*$ \\
\hline Do. & 1400 & 1,440 & --- & 1.6 & $*$ & $*$ & $*$ \\
\hline Do. & 1407 & 1,447 & 93 & -- & $*$ & $*$ & $*$ \\
\hline Do. & 1600 & 1,560 & $274(?)$ & 2.0 & $*$ & $*$ & $*$ \\
\hline Do. & 1700 & 1,620 & 120 & -- & $*$ & $*$ & $*$ \\
\hline
\end{tabular}


Table 35.--Data obtained from water samples collected during the May 24-25, 1978, injection test using playa-lake water

[Samples are from the point sampler designated 2-m red located $2 \mathrm{~m}$ radially from the injection well and $38.7 \mathrm{~m}$ below land surface. The dash (-) indicates sample is available but was not analyzed; the asterisk (*) indicates the sample was not analyzed and is no longer available.]

\begin{tabular}{|c|c|c|c|c|c|c|c|}
\hline \multicolumn{8}{|c|}{ 2-Meter red point sampler } \\
\hline \multirow{2}{*}{ Date } & \multirow{2}{*}{ Clock time } & \multirow{2}{*}{$\begin{array}{c}\text { Elapsed } \\
\text { time } \\
\text { (min) }\end{array}$} & \multirow{2}{*}{$\begin{array}{l}\text { Boron } \\
(\mu \mathrm{g} / \mathrm{L})\end{array}$} & \multirow{2}{*}{$\begin{array}{l}\text { Natural } \\
\text { organics } \\
\text { (mg C/L) }\end{array}$} & \multicolumn{3}{|c|}{ C/Co $\times 10^{5}$} \\
\hline & & & & & $\mathrm{F}-12$ & $\mathrm{BCF}$ & DBM \\
\hline $5-24-78$ & 1415 & 15 & -- & 0.5 & $*$ & $*$ & $*$ \\
\hline Do. & 1420 & 20 & -- & .5 & $*$ & $*$ & $*$ \\
\hline Do. & 1425 & 25 & 74 & -- & $*$ & $*$ & $*$ \\
\hline Do. & 1430 & 30 & -- & .5 & $*$ & $*$ & $*$ \\
\hline Do. & 1500 & 60 & -- & .6 & $*$ & 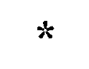 & $*$ \\
\hline Do. & 1510 & 70 & 85 & $-\cdots$ & $*$ & $*$ & $*$ \\
\hline Do. & 1515 & 75 & -- & .7 & $*$ & $*$ & $*$ \\
\hline Do. & 1520 & 80 & -- & .8 & $*$ & $*$ & $*$ \\
\hline Do. & 1525 & 85 & -- & .6 & $*$ & $*$ & $*$ \\
\hline Do. & 1530 & 90 & -- & .7 & $*$ & $*$ & $*$ \\
\hline Do. & 1540 & 100 & -- & .6 & $*$ & $*$ & $*$ \\
\hline Do. & 1550 & 110 & -- & .7 & $*$ & $*$ & $*$ \\
\hline Do. & 1600 & 120 & -- & 1.0 & $*$ & $*$ & $*$ \\
\hline Do. & 1610 & 130 & 122 & 1.6 & $*$ & $*$ & $*$ \\
\hline Do. & 1620 & 140 & -- & 1.4 & $*$ & $*$ & $*$ \\
\hline Do. & 1655 & 175 & --- & 1.6 & $*$ & $*$ & $*$ \\
\hline Do. & 1700 & 180 & -- & 1.6 & $*$ & $*$ & $*$ \\
\hline Do. & 1710 & 190 & 176 & 1.5 & $*$ & $*$ & $*$ \\
\hline Do. & 1720 & 200 & -- & 1.5 & $*$ & $*$ & $*$ \\
\hline Do. & 1730 & 210 & --- & 1.3 & $*$ & $*$ & $*$ \\
\hline Do. & 1740 & 220 & -- & 1.3 & $*$ & $*$ & $*$ \\
\hline Do. & 1800 & 240 & --- & 1.3 & $*$ & $*$ & $*$ \\
\hline Do. & 1815 & 255 & 174 & -- & $*$ & $*$ & $*$ \\
\hline Do. & 1830 & 270 & -- & 1.9 & $*$ & $*$ & $*$ \\
\hline Do. & 1845 & 285 & -- & 1.9 & $*$ & $*$ & $*$ \\
\hline
\end{tabular}


l'able 35.--Data obtained from water samples collected during the May 24-25, 1978, injection test using playa-lake water--Continued

\begin{tabular}{|c|c|c|c|c|c|c|c|}
\hline \multicolumn{8}{|c|}{ 2-Meter red point sampler } \\
\hline \multirow{2}{*}{ Date } & \multirow{2}{*}{ Clock time } & \multirow{2}{*}{$\begin{array}{c}\text { Elapsed } \\
\text { time } \\
(\min )\end{array}$} & \multirow{2}{*}{$\begin{array}{l}\text { Boron } \\
(\mu g / L)\end{array}$} & \multirow{2}{*}{$\begin{array}{l}\text { Natural } \\
\text { organics } \\
\text { (mg C/L) }\end{array}$} & \multicolumn{3}{|c|}{$\mathrm{C} / \mathrm{Co} \times 10^{5}$} \\
\hline & & & & & $\mathrm{F}-12$ & $\mathrm{BCF}$ & DBM \\
\hline $5-24-78$ & 1900 & 300 & -- & 1.5 & $*$ & $*$ & $\dot{*}$ \\
\hline Do. & 1920 & 320 & 172 & -- & $*$ & $*$ & $*$ \\
\hline Do. & 1930 & 330 & --- & 1.3 & $*$ & $*$ & $*$ \\
\hline Do. & 1940 & 340 & -- & 1.6 & $*$ & 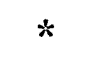 & $*$ \\
\hline Do. & 2000 & 360 & --- & 1.4 & * & $*$ & $*$ \\
\hline Do. & 2030 & 390 & 176 & 1.4 & $*$ & $*$ & $*$ \\
\hline Do. & 2040 & 400 & -- & 3.6 & $*$ & $*$ & $*$ \\
\hline Do. & 2100 & 420 & 185 & 1.6 & $*$ & $*$ & $*$ \\
\hline Do. & 2200 & 480 & --- & 2.0 & $*$ & $*$ & $*$ \\
\hline $5-25-78$ & 1100 & 1,260 & 222 & 1.6 & $*$ & $*$ & $*$ \\
\hline Do. & 1200 & 1,320 & -- & 3.5 & $*$ & $*$ & $*$ \\
\hline Do. & 1300 & 1,380 & 228 & 2.9 & $*$ & $*$ & $*$ \\
\hline Do. & 1400 & 1,440 & 237 & 3.4 & $*$ & $*$ & $*$ \\
\hline Do. & 1600 & 1,560 & --- & 2.0 & $*$ & $*$ & $*$ \\
\hline Do. & 1700 & 1,620 & 289 & 2.0 & $*$ & $*$ & $*$ \\
\hline
\end{tabular}


Table 36.--Data obtained from water samples collected during the May 24-25, 1978, injection test using playa-lake water

[Samples are from the point sampler designated 2-m orange located $34.8 \mathrm{~m}$ below land surface and $2 \mathrm{~m}$ radially from the injected well. The dash (-) indicates sample is available but was not analyzed; the asterisk (*) indicates the sample was not analyzed and is no longer available.]

\begin{tabular}{|c|c|c|c|c|c|c|c|}
\hline \multirow{3}{*}{ Date } & \multirow{3}{*}{ Clock time } & \multicolumn{4}{|c|}{ 2-Meter orange point sampler } & & \\
\hline & & \multirow{2}{*}{$\begin{array}{c}\text { Elapsed } \\
\text { time } \\
\text { (min) }\end{array}$} & \multirow{2}{*}{$\begin{array}{l}\text { Boron } \\
(\mu g / L)\end{array}$} & \multirow{2}{*}{$\begin{array}{c}\text { Natural } \\
\text { organics } \\
(\mathrm{mg} \mathrm{C} / \mathrm{L})\end{array}$} & \multicolumn{3}{|c|}{$\mathrm{C} /$ Co $\mathrm{X} 10^{5}$} \\
\hline & & & & & $\mathrm{F}-12$ & $\mathrm{BCF}$ & DBM \\
\hline $5-24-78$ & 1410 & 10 & 133 & 1.6 & $*$ & $*$ & $*$ \\
\hline Do. & 1415 & 15 & -- & 1.4 & $*$ & $*$ & $*$ \\
\hline Do. & 1435 & 35 & -- & 1.2 & $*$ & $*$ & $*$ \\
\hline Do. & 1445 & 45 & -- & 1.7 & $*$ & $*$ & $*$ \\
\hline Do. & 1455 & 55 & -- & 2.2 & $*$ & $*$ & $*$ \\
\hline Do. & 1500 & 60 & --- & 3.5 & $*$ & $*$ & $*$ \\
\hline Do. & 1505 & 65 & 478 & -- & $*$ & $*$ & $*$ \\
\hline Do. & 1510 & 70 & -- & 7 & $*$ & $*$ & $*$ \\
\hline Do. & 1520 & 80 & --- & 3.7 & $*$ & $*$ & $*$ \\
\hline Do. & 1530 & 90 & -- & 3.7 & $*$ & $*$ & $*$ \\
\hline Do. & 1550 & 110 & -- & 4.2 & $*$ & $*$ & $*$ \\
\hline Do. & 1600 & 120 & --- & 4.2 & $*$ & $*$ & $*$ \\
\hline Do. & 1610 & 130 & 732 & --- & $*$ & $*$ & $*$ \\
\hline Do. & 1620 & 140 & -- & 5.3 & $*$ & $*$ & $*$ \\
\hline Do. & 1655 & 175 & --- & 4.3 & $*$ & $*$ & $*$ \\
\hline Do. & 1700 & 180 & -- & 4.6 & $*$ & $*$ & $*$ \\
\hline Do. & 1710 & 190 & --- & 3.5 & $*$ & $\dot{t}$ & $*$ \\
\hline Do. & 1720 & 200 & -- & 3.6 & $*$ & $*$ & $*$ \\
\hline Do. & 1730 & 210 & -- & 3.6 & $*$ & $*$ & $*$ \\
\hline Do. & 1740 & 220 & -- & 3.6 & $*$ & $*$ & $*$ \\
\hline Do. & 1750 & 230 & --- & 3.8 & $*$ & $*$ & $*$ \\
\hline Do. & 1800 & 240 & --- & 4.7 & $*$ & $*$ & $*$ \\
\hline Do. & 1815 & 255 & --- & 4.8 & $*$ & $*$ & $*$ \\
\hline Do. & 1830 & 270 & -- & 4.4 & $*$ & $*$ & $*$ \\
\hline Do. & 1845 & 285 & -- & 4.2 & $*$ & $*$ & $*$ \\
\hline
\end{tabular}


Table 36.--Data obtained from water samples collected during the May 24-25, 1978, injection test using playa-lake water--Continued

\begin{tabular}{|c|c|c|c|c|c|c|c|}
\hline \multicolumn{8}{|c|}{ 2-Meter orange point sampler } \\
\hline \multirow{2}{*}{ Date } & \multirow{2}{*}{ Clock time } & \multirow{2}{*}{$\begin{array}{c}\text { Elapsed } \\
\text { time } \\
\text { (min) }\end{array}$} & \multirow{2}{*}{$\begin{array}{l}\text { Boron } \\
(\mu \mathrm{g} / \mathrm{L})\end{array}$} & \multirow{2}{*}{$\begin{array}{c}\text { Natural } \\
\text { organics } \\
\text { (mg C/L) }\end{array}$} & \multicolumn{3}{|c|}{$\mathrm{C} / \mathrm{Co} \times 10^{5}$} \\
\hline & & & & & $\mathrm{F}-12$ & $\mathrm{BCF}$ & $\mathrm{DBM}$ \\
\hline $5-24-78$ & 1900 & 300 & --- & 4.8 & $*$ & $*$ & $*$ \\
\hline Do. & 1920 & 320 & --- & 4.4 & $*$ & $*$ & $*$ \\
\hline Do. & 1940 & 340 & -- & 4.7 & $*$ & $*$ & $*$ \\
\hline Do. & 2000 & 360 & --- & 4.4 & $*$ & $*$ & $*$ \\
\hline Do. & 2030 & 390 & --- & 4.2 & $*$ & * & $*$ \\
\hline Do. & 2100 & 420 & --- & 4.2 & $*$ & $*$ & $*$ \\
\hline Do. & 2200 & 480 & --- & 4.3 & $\star$ & $*$ & $*$ \\
\hline $5-25-78$ & 1100 & 1,260 & -- & 3.6 & $*$ & $*$ & $*$ \\
\hline Do. & 1300 & 1,380 & --- & 4 & $*$ & $亡$ & $*$ \\
\hline Do. & 1400 & 1,440 & --- & 4 & $*$ & $*$ & $*$ \\
\hline Do. & 1401 & 1,441 & 946 & -- & $*$ & $*$ & $*$ \\
\hline Do. & $(1409-1415)$ & $1,449-1,455$ & --- & (4) & $*$ & $*$ & $*$ \\
\hline Do. & 1600 & 1,560 & 974 & $4 \cdot 2$ & $*$ & $*$ & $*$ \\
\hline Do. & 1700 & 1,620 & 1,061 & 4.3 & $*$ & $*$ & $*$ \\
\hline
\end{tabular}


Table 37.--Data obtained from water samples collected during the May 24-25, 1978, injection test using playa-lake water

[Samples are from the point sampler designated 2-m green located $2 \mathrm{~m}$ radially from the injection well and $32.6 \mathrm{~m}$ below land surface. The dash (-) indicates sample is available but was not analyzed; the asterisk (*) indicates the sample was not analyzed and is no longer available.]

\begin{tabular}{|c|c|c|c|c|c|c|c|}
\hline \multirow{3}{*}{ Date } & \multirow{3}{*}{ Clock time } & \multicolumn{4}{|c|}{ 2-Meter green point sampler } & & \\
\hline & & Elapsed & Boron & Natura 1 & \multicolumn{3}{|c|}{$\mathrm{C} / \mathrm{Co} \times 10^{5}$} \\
\hline & & $(\min )$ & & $(\mathrm{mg} \mathrm{C} / \mathrm{L})$ & $\mathrm{F}-12$ & $\mathrm{BCF}$ & DBM \\
\hline $5-24-78$ & 1410 & 10 & 185 & --- & $*$ & $*$ & $*$ \\
\hline Do. & 1415 & 15 & 154 & 1.2 & $*$ & $*$ & $*$ \\
\hline Do. & 1425 & 25 & -- & 1.2 & $*$ & $*$ & $*$ \\
\hline Do. & 1435 & 35 & -- & .8 & $*$ & $*$ & $*$ \\
\hline Do. & 1445 & 45 & -- & .8 & $*$ & $*$ & $*$ \\
\hline Do. & 1455 & 55 & -- & .8 & $*$ & $*$ & $*$ \\
\hline Do. & 1500 & 60 & --- & .8 & $*$ & $*$ & $*$ \\
\hline Do. & 1510 & 70 & $-\cdots$ & 1.0 & $*$ & $*$ & $*$ \\
\hline Do. & 1515 & 75 & 165 & $-\cdots$ & $*$ & $*$ & $*$ \\
\hline Do. & 1520 & 80 & -- & .9 & $*$ & $*$ & $*$ \\
\hline Do. & 1530 & 90 & -- & .9 & $*$ & $\star$ & $*$ \\
\hline Do. & 1540 & 100 & --- & .9 & $*$ & $*$ & $*$ \\
\hline Do. & 1550 & 110 & -- & 1.0 & $*$ & $*$ & $*$ \\
\hline Do. & 1600 & 120 & -- & 1.9 & $*$ & $*$ & $*$ \\
\hline Do. & 1610 & 130 & 170 & --- & $*$ & $*$ & $*$ \\
\hline Do. & 1620 & 140 & -- & .9 & $*$ & $\frac{1}{*}$ & $\stackrel{1}{\star}$ \\
\hline Do. & 1655 & 175 & --- & .9 & $*$ & $*$ & $*$ \\
\hline Do. & 1700 & 180 & -- & 1.3 & $*$ & $\stackrel{*}{*}$ & $*$ \\
\hline Do. & 1710 & 190 & $-\cdots$ & 1.3 & $*$ & $*$ & $*$ \\
\hline Do. & 1720 & 200 & -- & .9 & $*$ & $*$ & $*$ \\
\hline Do. & 1740 & 220 & --- & 1.2 & $*$ & $*$ & $*$ \\
\hline Do. & 1800 & 240 & -- & 1.6 & $*$ & $*$ & $\frac{1}{*}$ \\
\hline Do. & 1815 & 255 & -- & 1.1 & $*$ & $*$ & $*$ \\
\hline Do. & 1830 & 270 & -- & 2.3 & $*$ & $*$ & $*$ \\
\hline
\end{tabular}


Table 37.--Data obtained from water samples collected during the May 24-25, 1978, injection test using playa-lake water--Continued

\begin{tabular}{|c|c|c|c|c|c|c|c|}
\hline \multicolumn{8}{|c|}{ 2-Meter green point sampler } \\
\hline \multirow{2}{*}{ Date } & \multirow{2}{*}{ Clock time } & \multirow{2}{*}{$\begin{array}{c}\text { Elapsed } \\
\text { time } \\
\text { (min) }\end{array}$} & \multirow{2}{*}{$\begin{array}{l}\text { Boron } \\
(\mu \mathrm{g} / \mathrm{L})\end{array}$} & \multirow{2}{*}{$\begin{array}{l}\text { Natural } \\
\text { organics } \\
\text { (mg C/L) }\end{array}$} & \multicolumn{3}{|c|}{$\mathrm{C} / \mathrm{Co} \times 10^{5}$} \\
\hline & & & & & $\mathrm{F}-12$ & $\mathrm{BCF}$ & DBM \\
\hline $5-24-78$ & 1845 & 285 & -- & 0.8 & $*$ & $*$ & $*$ \\
\hline Do. & 1900 & 300 & --- & 1.1 & $*$ & $*$ & $*$ \\
\hline Do. & 1920 & 320 & -- & 1.2 & $*$ & $*$ & $*$ \\
\hline Do. & 1940 & 340 & -- & 2.0 & $*$ & $*$ & $*$ \\
\hline Do. & 2000 & 360 & -- & 1.1 & $*$ & $*$ & $*$ \\
\hline Do. & 2030 & 390 & -- & 1.6 & $*$ & $*$ & $*$ \\
\hline Do. & 2100 & 420 & -- & .9 & $*$ & $*$ & $*$ \\
\hline Do. & 2200 & 480 & -- & 1.5 & $*$ & $*$ & $*$ \\
\hline Do. & 1200 & 1,320 & --- & 1.5 & $*$ & $*$ & $*$ \\
\hline Do. & 1300 & 1,380 & 250 & 1.1 & $*$ & $*$ & $*$ \\
\hline Do. & 1400 & 1,440 & -- & 1.3 & $*$ & $*$ & $*$ \\
\hline Do. & 1407 & 1,447 & 287 & 1.2 & $*$ & $*$ & $*$ \\
\hline Do. & 1600 & 1,560 & 339 & 1.6 & $*$ & $*$ & $*$ \\
\hline Do. & 1700 & 1,620 & 354 & 1.4 & $*$ & $*$ & $*$ \\
\hline Do. & 1730 & 1,650 & 348 & --- & $*$ & $*$ & $*$ \\
\hline
\end{tabular}


Table 38.--Data obtained from water samples collected during the May 24-25, 1978, injection test using playa-lake water

[Samples are from the observation well located $5 \mathrm{~m}$ from the injection well. The 5-m well is screened for the full-saturated thickness of the aquifer. The dash (-) indicates sample is available but was not analyzed; the asterisk $(*)$ indicates the sample was not analyzed and is no longer available.]

5-Meter wel1

\begin{tabular}{|c|c|c|c|c|c|c|c|}
\hline \multirow{2}{*}{ Date } & \multirow{2}{*}{ Clock time } & \multirow{2}{*}{$\begin{array}{c}\text { Elapsed } \\
\text { time } \\
(\min )\end{array}$} & \multirow{2}{*}{$\begin{array}{l}\text { Boron } \\
(\mu \mathrm{g} / \mathrm{L})\end{array}$} & \multirow{2}{*}{$\begin{array}{l}\text { Natural } \\
\text { organics } \\
(\mathrm{mg} \mathrm{C} / \mathrm{L})\end{array}$} & \multicolumn{3}{|c|}{$\mathrm{C} / \mathrm{Co} \times 10^{5}$} \\
\hline & & & & & $\mathrm{F}-12$ & $\mathrm{BCF}$ & DBM \\
\hline $5-24-78$ & 1401 & 1 & 90 & 1 & 6.4 & 1.5 & 0 \\
\hline Do. & 1410 & 10 & --- & .6 & 6.4 & 1.4 & 0 \\
\hline Do. & 1422 & 22 & --- & .7 & $*$ & $*$ & $*$ \\
\hline Do. & 1430 & 30 & --- & --- & 6.4 & 1.5 & 0 \\
\hline Do. & 1440 & 40 & -- & .9 & 7.4 & 1.8 & 0 \\
\hline Do. & 1445 & 45 & --- & --- & 16 & 190 & 7.9 \\
\hline Do. & 1451 & 51 & --- & -- & 110 & 880 & 31 \\
\hline Do. & 1501 & 61 & --- & 1.2 & 210 & 1,400 & 280 \\
\hline Do. & 1506 & 66 & --- & 3.2 & $\dot{*}$ & $*$ & $\star$ \\
\hline Do. & 1511 & 71 & --- & 1.5 & $*$ & $*$ & $*$ \\
\hline Do. & 1516 & 76 & 250 & 1.7 & $*$ & $*$ & $*$ \\
\hline Do. & 1522 & 81 & --- & 1.8 & $*$ & $*$ & $*$ \\
\hline Do. & 1532 & 92 & --- & 2.35 & 860 & 2,000 & 570 \\
\hline Do. & 1542 & 102 & --- & 3.1 & $*$ & $*$ & $*$ \\
\hline Do. & 1552 & 112 & --- & 3.6 & $*$ & $*$ & $*$ \\
\hline Do. & 1601 & 121 & --- & 2.8 & 1,100 & 2,700 & 660 \\
\hline Do. & 1611 & 131 & --- & 3 & $*$ & $*$ & $*$ \\
\hline Do. & 1620 & 140 & --- & 4 & $*$ & $*$ & $*$ \\
\hline Do. & 1640 & 160 & --- & 2 & 280 & 1,500 & 580 \\
\hline Do. & 1700 & 180 & 360 & 2 & $*$ & $*$ & $\star$ \\
\hline Do. & 1712 & 192 & --- & 2.1 & $*$ & $*$ & $*$ \\
\hline Do. & 1715 & 195 & --- & -- & 140 & 400 & 780 \\
\hline Do. & 1726 & 206 & --- & 2.6 & $*$ & $*$ & $*$ \\
\hline
\end{tabular}


Table 38.--Data obtained from water samples collected during the May 24-25, 1978, injection test using playa-lake water--Continued

\begin{tabular}{|c|c|c|c|c|c|c|c|}
\hline \multicolumn{8}{|c|}{ 5-Meter we11 } \\
\hline \multirow{2}{*}{ Date } & \multirow{2}{*}{ Clock time } & \multirow{2}{*}{$\begin{array}{c}\text { Elapsed } \\
\text { time } \\
\text { (min) }\end{array}$} & \multirow{2}{*}{$\begin{array}{l}\text { Boron } \\
(\mu \mathrm{g} / \mathrm{L})\end{array}$} & \multirow{2}{*}{$\begin{array}{l}\text { Natural } \\
\text { organics } \\
\text { (mg C/L) }\end{array}$} & \multicolumn{3}{|c|}{$\mathrm{C} / \mathrm{Co} \times 10^{5}$} \\
\hline & & & & & $\mathrm{F}-12$ & $\mathrm{BCF}$ & DBM \\
\hline $5-24-78$ & 1741 & 221 & --- & 2.2 & 2,800 & 3,100 & 940 \\
\hline Do. & 1803 & 243 & --- & 2.4 & $*$ & $*$ & $*$ \\
\hline Do. & 1815 & 255 & -- & 3 & $*$ & $*$ & $*$ \\
\hline Do. & 1830 & 270 & --- & 3.1 & $*$ & * & $*$ \\
\hline Do. & 1850 & 290 & --- & 2.3 & $*$ & $*$ & $*$ \\
\hline Do. & 1856 & 296 & 540 & 2.6 & 2,100 & 3,200 & 820 \\
\hline Do. & 1936 & 336 & --- & 1.7 & $*$ & $*$ & $*$ \\
\hline Do. & 2000 & 360 & --- & 2.3 & 1,500 & 2,800 & 840 \\
\hline Do. & 2020 & 380 & --- & 3.1 & $*$ & $*$ & $*$ \\
\hline Do. & 2040 & 400 & --- & 3 & $*$ & $*$ & $*$ \\
\hline Do. & 2104 & 424 & 630 & 2.6 & $*$ & $*$ & $*$ \\
\hline Do. & 2135 & 455 & --- & 2.4 & $*$ & $*$ & $*$ \\
\hline Do & 2205 & 485 & --- & 2.6 & $*$ & $*$ & $*$ \\
\hline $5-25-78$ & 1105 & 1,265 & 670 & 2.6 & 5,000 & 4,400 & 1,300 \\
\hline Do. & 1200 & 1,320 & --- & 3.3 & $*$ & $*$ & $*$ \\
\hline Do. & 1400 & 1,440 & 650 & 3.4 & $*$ & $*$ & $*$ \\
\hline Do. & 1600 & 1,560 & --- & 3.2 & $*$ & $*$ & $*$ \\
\hline Do. & 1700 & 1,620 & 710 & 3.1 & $*$ & $*$ & $*$ \\
\hline
\end{tabular}


Table 39.--Data obtained from water samples collected during the May 24-25, 1978, injection test using playa-lake water

[Samples are from the point sampler designated 5-m black located $5 \mathrm{~m}$ radially from the injection well and $41.8 \mathrm{~m}$ below land surface. The dash (-) indicates sample is available but was not analyzed; the asterisk (*) indicates the sample was not analyzed and is no longer available.]

\begin{tabular}{|c|c|c|c|c|c|c|c|}
\hline \multirow{3}{*}{ Date } & \multirow{3}{*}{ Clock time } & \multicolumn{4}{|c|}{ 5-Meter black point sampler } & & \\
\hline & & & Boron & Natural & \multicolumn{3}{|c|}{$\mathrm{C} / \mathrm{Co} \times 10^{5}$} \\
\hline & & (min) & (1) & $(\mathrm{mg} \mathrm{C} / \mathrm{L})$ & $\mathrm{F}-12$ & $\mathrm{BCF}$ & DBM \\
\hline $5-24-78$ & 1400 & 0 & 113 & --- & $*$ & $*$ & $*$ \\
\hline Do. & 1430 & 30 & --- & 1.1 & $*$ & $*$ & $*$ \\
\hline Do. & 1500 & 60 & --- & 1.0 & $*$ & $*$ & $*$ \\
\hline Do. & 1505 & 65 & --- & 1.0 & $*$ & $*$ & $*$ \\
\hline Do. & 1510 & 70 & --- & 1.0 & $*$ & $*$ & $*$ \\
\hline Do. & 1515 & 75 & --- & 1.1 & $*$ & 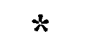 & $*$ \\
\hline Do. & 1525 & 85 & --- & .8 & $\star$ & $*$ & $*$ \\
\hline Do. & 1535 & 95 & --- & 1.0 & $*$ & $*$ & $*$ \\
\hline Do. & 1540 & 100 & --- & 1.0 & $*$ & $*$ & $*$ \\
\hline Do. & 1545 & 105 & --- & 1.1 & $*$ & $*$ & $*$ \\
\hline Do. & 1555 & 115 & 122 & 1.0 & $*$ & $*$ & $*$ \\
\hline Do. & 1600 & 120 & --- & 1.8 & $*$ & $*$ & $*$ \\
\hline Do. & 1605 & 125 & --- & 1.0 & $*$ & $*$ & $*$ \\
\hline Do. & 1610 & 130 & --- & 1.6 & $*$ & $*$ & $*$ \\
\hline Do. & 1615 & 135 & --- & 1.0 & $*$ & $*$ & $*$ \\
\hline Do. & 1620 & 140 & --- & 1.0 & $*$ & $\star$ & $*$ \\
\hline Do. & 1655 & 175 & --- & .8 & $*$ & $*$ & $*$ \\
\hline Do. & 1700 & 180 & -- & 1.0 & $*$ & 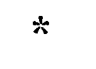 & $*$ \\
\hline Do. & 1705 & 185 & --- & .8 & $*$ & $*$ & $*$ \\
\hline Do. & 1710 & 190 & --- & 1.0 & $*$ & $*$ & $*$ \\
\hline Do. & 1715 & 195 & -- & 1.1 & $*$ & $*$ & $*$ \\
\hline Do. & 1720 & 200 & --- & 1.1 & $*$ & $*$ & $*$ \\
\hline Do. & 1725 & 205 & --- & 2.4 & $*$ & $*$ & $*$ \\
\hline Do. & 1730 & 210 & --- & 1.1 & $*$ & $*$ & $*$ \\
\hline Do. & 1735 & 215 & --- & 1.3 & $*$ & $*$ & $*$ \\
\hline
\end{tabular}


Table 39.--Data obtained from water samples collected during the May 24-25, 1978, injection test using playa-lake water--Continued

\begin{tabular}{|c|c|c|c|c|c|c|c|}
\hline \multicolumn{8}{|c|}{ 5-Meter black point sampler } \\
\hline \multirow{2}{*}{ Date } & \multirow{2}{*}{ Clock time } & \multirow{2}{*}{$\begin{array}{c}\text { Elapsed } \\
\text { time } \\
\text { (min) }\end{array}$} & \multirow{2}{*}{$\begin{array}{l}\text { Boron } \\
(\mu \mathrm{g} / \mathrm{L})\end{array}$} & \multirow{2}{*}{$\begin{array}{l}\text { Natural } \\
\text { organics } \\
\text { (mg C/L) }\end{array}$} & \multicolumn{3}{|c|}{$\mathrm{C} / \mathrm{Co} \times 10^{5}$} \\
\hline & & & & & $\mathrm{F}-12$ & $\mathrm{BCF}$ & DBM \\
\hline $5-24-78$ & 1740 & 220 & -- & 2.3 & $*$ & $*$ & $*$ \\
\hline Do. & 1750 & 230 & -- & 1.3 & $\star$ & $*$ & $*$ \\
\hline Do. & 1755 & 235 & 122 & 1.5 & $*$ & $*$ & $*$ \\
\hline Do. & 1800 & 240 & -- & 1.5 & $*$ & $*$ & $*$ \\
\hline Do. & 1810 & 250 & -- & 1.7 & $*$ & $*$ & $\stackrel{ \pm}{\star}$ \\
\hline Do. & 1820 & 260 & -- & 1.5 & $*$ & $*$ & $*$ \\
\hline Do. & 1830 & 270 & --- & 2.0 & $*$ & $*$ & $*$ \\
\hline Do. & 1840 & 280 & -- & 2.0 & $*$ & $*$ & $*$ \\
\hline Do. & 1850 & 290 & -- & 2.0 & $*$ & $*$ & $*$ \\
\hline Do. & 1900 & 300 & -- & 1.9 & $*$ & $*$ & $*$ \\
\hline Do. & 1915 & 315 & -- & 1.8 & $*$ & $*$ & $*$ \\
\hline Do. & 1930 & 330 & -- & 1.9 & $*$ & $*$ & $*$ \\
\hline Do. & 1945 & 345 & -- & 1.6 & $*$ & $*$ & * \\
\hline Do. & 2000 & 360 & -- & 1.6 & $*$ & $*$ & $*$ \\
\hline Do. & 2020 & 380 & 118 & 1.6 & $*$ & $*$ & $\star$ \\
\hline Do. & 2040 & 400 & -- & 1.7 & 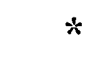 & $*$ & $*$ \\
\hline Do. & 2200 & 480 & 200 & -- & $*$ & $*$ & $*$ \\
\hline $5-25-78$ & 1100 & 1,260 & 116 & -- & $*$ & $*$ & $*$ \\
\hline Do. & 1200 & 1,320 & 116 & -- & $*$ & $*$ & $*$ \\
\hline Do. & 1400 & 1,440 & 116 & 1.9 & $*$ & $*$ & $*$ \\
\hline Do. & 1700 & 1,620 & 116 & -- & $*$ & $*$ & $*$ \\
\hline
\end{tabular}


Table 40.--Data obtained from water samples collected during the May 24-25, 1978, injection test using playa-lake water

[Samples are from an observation well designated 5-m red and screened only for $0.7 \mathrm{~m}$ at $40.0 \mathrm{~m}$ below land surface. This sampler is located at a distance of $5 \mathrm{~m}$ radially from the injection well. The dash (-) indicates sample is available but was not analyzed; the asterisk (*) indicates the sample was not analyzed and is no longer available.]

\begin{tabular}{|c|c|c|c|c|c|c|c|}
\hline \multirow{3}{*}{ Date } & \multirow{3}{*}{ Clock time } & \multicolumn{4}{|c|}{ 5-Meter red point sampler } & & \\
\hline & & \multirow{2}{*}{$\begin{array}{l}\text { Elapsed } \\
\text { time } \\
\text { (min) }\end{array}$} & \multirow{2}{*}{$\begin{array}{l}\text { Boron } \\
(\mu \mathrm{g} / \mathrm{L})\end{array}$} & \multirow{2}{*}{$\begin{array}{l}\text { Natural } \\
\text { organics } \\
\text { (mg C/L) }\end{array}$} & \multicolumn{3}{|c|}{$\mathrm{C} / \mathrm{Co} \times 10^{5}$} \\
\hline & & & & & $\mathrm{F}-12$ & $\mathrm{BCF}$ & DBM \\
\hline $5-24-78$ & 1430 & 30 & -- & 0.8 & $*$ & $*$ & $*$ \\
\hline Do. & 1440 & 40 & 277 & 2.0 & $*$ & $*$ & $*$ \\
\hline Do. & 1450 & 50 & --- & 3.5 & $*$ & $*$ & $*$ \\
\hline Do. & 1500 & 60 & -- & 2.8 & $*$ & $*$ & $\stackrel{2}{*}$ \\
\hline Do. & 1505 & 65 & 476 & 3.0 & $*$ & $*$ & $*$ \\
\hline Do. & 1510 & 70 & --- & 3.4 & $*$ & $*$ & $*$ \\
\hline Do. & 1515 & 75 & -- & 3.4 & $*$ & $*$ & $*$ \\
\hline Do. & 1520 & 80 & -- & 3.9 & $*$ & $*$ & $*$ \\
\hline Do. & 1525 & 85 & -- & 3.9 & $*$ & $*$ & $*$ \\
\hline Do. & 1530 & 90 & --- & 3.9 & $*$ & $*$ & $*$ \\
\hline Do. & 1540 & 100 & -- & 4.8 & $*$ & $*$ & $*$ \\
\hline Do. & 1545 & 105 & --- & 4.5 & $*$ & $*$ & $*$ \\
\hline Do. & 1550 & 110 & --- & 4.0 & $*$ & $*$ & $*$ \\
\hline Do. & 1555 & 115 & --- & 4.8 & $*$ & $*$ & $*$ \\
\hline Do. & 1600 & 120 & -- & 4.7 & $*$ & $*$ & $*$ \\
\hline Do. & 1605 & 125 & -- & 4.4 & $*$ & $*$ & $*$ \\
\hline Do. & 1610 & 130 & -- & 4.5 & $*$ & $*$ & $*$ \\
\hline Do. & 1620 & 140 & --- & 4.5 & $*$ & $*$ & $*$ \\
\hline Do. & 1655 & 175 & --- & 4.5 & $*$ & $*$ & $*$ \\
\hline Do. & 1700 & 180 & -- & 3.8 & $*$ & \pm & $*$ \\
\hline Do. & 1705 & 185 & 449 & 2.6 & $*$ & $*$ & $*$ \\
\hline Do. & 1710 & 190 & --- & 2.8 & $*$ & $*$ & $*$ \\
\hline Do. & 1715 & 195 & -- & 3.1 & $*$ & $*$ & $*$ \\
\hline Do. & 1720 & 200 & $-\ldots$ & 3.9 & $*$ & $*$ & $*$ \\
\hline
\end{tabular}


Table 40.--Data obtained from water samples collected during the May 24-25, 1978, injection test using playa-lake water--Continued

\begin{tabular}{|c|c|c|c|c|c|c|c|}
\hline \multirow{3}{*}{ Date } & \multirow{3}{*}{ Clock time } & \multicolumn{4}{|c|}{ 5-Meter red point sampler } & & \\
\hline & & \multirow{2}{*}{$\begin{array}{l}\text { Elapsed } \\
\text { time } \\
\text { (min) }\end{array}$} & \multirow{2}{*}{$\begin{array}{l}\text { Boron } \\
(\mu \mathrm{g} / \mathrm{L})\end{array}$} & \multirow{2}{*}{$\begin{array}{l}\text { Natural } \\
\text { organics } \\
\text { (mg C/L) }\end{array}$} & \multicolumn{3}{|c|}{$\mathrm{C} /$ Co $\times 10^{5}$} \\
\hline & & & & & $\mathrm{F}-12$ & $\mathrm{BCF}$ & DBM \\
\hline $5-24-78$ & 1725 & 205 & -- & 3.5 & $*$ & $*$ & $*$ \\
\hline Do. & 1730 & 210 & --- & 4.4 & $*$ & $*$ & $*$ \\
\hline Do. & 1735 & 215 & --- & 4.1 & $*$ & $*$ & $*$ \\
\hline Do. & 1740 & 220 & -- & 4.1 & $*$ & $*$ & $*$ \\
\hline Do. & 1750 & 230 & --- & 4.7 & $\star$ & $*$ & $*$ \\
\hline Do. & 1755 & 235 & --- & 3.5 & $*$ & $*$ & $*$ \\
\hline Do. & 1820 & 260 & --- & 3.4 & $*$ & $*$ & $*$ \\
\hline Do. & 1850 & 290 & -- & 4.4 & $*$ & $*$ & $*$ \\
\hline Do. & 1915 & 315 & 856 & 4.3 & $*$ & $*$ & $*$ \\
\hline Do. & 2020 & 380 & -- & 3.0 & $*$ & $*$ & $*$ \\
\hline Do. & 2040 & 400 & 491 & --- & $*$ & $*$ & $*$ \\
\hline Do. & 2130 & 450 & 558 & --- & $*$ & $*$ & $*$ \\
\hline Do. & 2200 & 480 & -- & 3.5 & $*$ & $*$ & $*$ \\
\hline $5-25-78$ & 1115 & 1,275 & 602 & --- & $*$ & $*$ & $*$ \\
\hline Do. & 1200 & 1,320 & 637 & --- & $*$ & $*$ & $*$ \\
\hline Do. & 1400 & 1,440 & 754 & --- & $*$ & $*$ & $*$ \\
\hline Do. & 1600 & 1,560 & 664 & --- & $*$ & $*$ & $*$ \\
\hline Do. & 1700 & 1,620 & 891 & -- & $*$ & $*$ & $*$ \\
\hline
\end{tabular}


Table 41.--Data obtained from water samples collected during the May 24-25, 1978, injection test using playa-lake water

[Samples are from the point sampler designated 5-m orange located $37.5 \mathrm{~m}$ below land surface and $5 \mathrm{~m}$ radially from the injection well. The dash (-) indicates sample is available but was not analyzed; an asterisk (*) indicates the sample was not analyzed and is no longer available.]

\begin{tabular}{|c|c|c|c|c|c|c|c|}
\hline \multirow{3}{*}{ Date } & \multirow{3}{*}{ Clock time } & \multicolumn{4}{|c|}{ 5-Meter orange point sampler } & & \\
\hline & & Elapsed & Boron & Natural & \multicolumn{3}{|c|}{$\mathrm{C} / \mathrm{Co} \times 10^{5}$} \\
\hline & & (min) & & $(\mathrm{mg} \mathrm{C} / \mathrm{L})$ & $\mathrm{F}-12$ & $\mathrm{BCF}$ & DBM \\
\hline $5-24-78$ & 1400 & 0 & -- & 0.6 & $*$ & $*$ & $*$ \\
\hline Do. & 1410 & 10 & 100 & -- & $*$ & $*$ & $*$ \\
\hline Do. & 1420 & 20 & -- & .6 & $*$ & $*$ & $*$ \\
\hline Do. & 1440 & 40 & -- & .7 & $*$ & $*$ & $*$ \\
\hline Do. & 1505 & 65 & 90 & .6 & $*$ & $*$ & $*$ \\
\hline Do. & 1530 & 90 & -- & .8 & $*$ & $*$ & $*$ \\
\hline Do. & 1545 & 105 & -- & .9 & $*$ & $*$ & $*$ \\
\hline Do. & 1600 & 120 & -- & 1.5 & $*$ & $*$ & $*$ \\
\hline Do. & 1605 & 125 & 120 & -- & $*$ & $*$ & $*$ \\
\hline Do. & 1615 & 135 & -- & 2.6 & $*$ & $*$ & $*$ \\
\hline Do. & 1655 & 175 & -- & 2.3 & $*$ & $*$ & $*$ \\
\hline Do. & 1705 & 185 & 120 & --- & $*$ & $*$ & $*$ \\
\hline Do. & 1710 & 190 & -- & 2.9 & $*$ & $*$ & $*$ \\
\hline Do. & 1735 & 215 & -- & 2.6 & $*$ & $*$ & $*$ \\
\hline Do. & 1750 & 230 & --- & 2.8 & $*$ & $*$ & $*$ \\
\hline Do. & 1755 & 235 & 150 & --- & * & $\star$ & $*$ \\
\hline Do. & 1800 & 240 & -- & 2.6 & $*$ & $*$ & $*$ \\
\hline Do. & 1820 & 260 & -- & 2.7 & $*$ & $*$ & $*$ \\
\hline Do. & 1840 & 280 & -- & 2.6 & $*$ & $*$ & $*$ \\
\hline Do. & 1850 & 290 & 160 & -- & $*$ & $*$ & $*$ \\
\hline Do. & 1900 & 300 & -- & 2.9 & $*$ & $*$ & $*$ \\
\hline Do. & 1930 & 330 & --- & 3.1 & $*$ & $*$ & $*$ \\
\hline Do. & 2020 & 380 & 170 & 3.2 & $*$ & $*$ & $*$ \\
\hline Do. & 2200 & 480 & -- & 3 & $*$ & $*$ & $*$ \\
\hline
\end{tabular}


Table 41.--Data obtained from water samples collected during the May 24-25, 1978, injection test using playa-lake water-Continued

\begin{tabular}{|c|c|c|c|c|c|c|c|}
\hline \multicolumn{8}{|c|}{ 5-Meter orange point sampler } \\
\hline \multirow{2}{*}{ Date } & \multirow{2}{*}{ Clock time } & \multirow{2}{*}{$\begin{array}{c}\text { Elapsed } \\
\text { time } \\
\text { (min) }\end{array}$} & \multirow{2}{*}{$\begin{array}{l}\text { Boron } \\
(\mu \mathrm{g} / \mathrm{L})\end{array}$} & \multirow{2}{*}{$\begin{array}{l}\text { Natural } \\
\text { organics } \\
(\mathrm{mg} \mathrm{C} / \mathrm{L})\end{array}$} & \multicolumn{3}{|c|}{$\mathrm{C} / \mathrm{Co} \times 10^{5}$} \\
\hline & & & & & $\mathrm{F}-12$ & $\mathrm{BCF}$ & DBM \\
\hline $5-25-78$ & 1100 & 1,260 & 120 & -- & $*$ & $*$ & $*$ \\
\hline Do. & 1400 & 1,440 & 140 & -- & $*$ & * & $*$ \\
\hline Do. & 1600 & 1,560 & 140 & -- & $*$ & $*$ & $*$ \\
\hline Do. & 1700 & 1,620 & 150 & $-\cdots$ & * & $*$ & $*$ \\
\hline
\end{tabular}


Table 42.--Data obtained from water samples collected during the May 24-25, 1978, injection test using playa-lake water

[Samples are from the observation well $10 \mathrm{~m}$ from the injection well and are from the zone screened only from 41.5 to $42.7 \mathrm{~m}$ below land surface. The dash (-) indicates sample is available but was not analyzed; an asterisk $(*)$ indicates the sample was not analyzed and is no longer available.]

\begin{tabular}{|c|c|c|c|c|c|c|c|}
\hline \multicolumn{8}{|c|}{ 10-Meter point sampler } \\
\hline \multirow{2}{*}{ Date } & \multirow{2}{*}{ Clock time } & \multirow{2}{*}{$\begin{array}{l}\text { Elapsed } \\
\text { time } \\
\text { (min) }\end{array}$} & \multirow{2}{*}{$\begin{array}{l}\text { Boron } \\
(\mu \mathrm{g} / \mathrm{L})\end{array}$} & \multirow{2}{*}{$\begin{array}{l}\text { Natural } \\
\text { organics } \\
(\mathrm{mg} \mathrm{C} / \mathrm{L})\end{array}$} & \multicolumn{3}{|c|}{$\mathrm{C} / \mathrm{Co} \times 10^{5}$} \\
\hline & & & & & $F-12$ & $\mathrm{BCF}$ & DBM \\
\hline $5-24-78$ & 1411 & 11 & 80 & --- & 6.4 & 1.4 & 0 \\
\hline Do. & 1445 & 45 & --- & 0.6 & $*$ & $*$ & $*$ \\
\hline Do. & 1540 & 90 & --- & --- & 6.4 & 0 & 0 \\
\hline Do. & 1645 & 165 & 80 & .6 & 6.4 & 1.4 & 0 \\
\hline Do. & 1745 & 225 & --- & .6 & 14 & 3.6 & 1 \\
\hline Do. & 1859 & 229 & 80 & --- & 32 & 100 & 8 \\
\hline Do. & 2001 & 361 & --- & 1.1 & 66 & 84 & 17 \\
\hline Do. & 2031 & 391 & 100 & 1.2 & 88 & 220 & 43 \\
\hline Do. & 2135 & 455 & --- & 1.3 & $*$ & $*$ & $*$ \\
\hline Do. & 2205 & 485 & 130 & --- & $*$ & $*$ & $*$ \\
\hline $5-25-78$ & 1135 & 1,296 & 150 & --- & $*$ & $*$ & $*$ \\
\hline Do. & 1250 & 1,370 & --- & --- & 170 & 1,200 & 320 \\
\hline Do. & 1400 & 1,440 & 170 & --- & $*$ & $*$ & 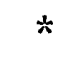 \\
\hline Do. & 1600 & 1,560 & 180 & --- & $*$ & $*$ & $*$ \\
\hline Do. & 1710 & 1,630 & 270 & --- & $*$ & $*$ & $*$ \\
\hline
\end{tabular}


Table 43.--Data obtained from water samples collected during the May 24-25, 1978, injection test using playa-lake water

[Samples are from the observation well $15 \mathrm{~m}$ from the injection well, which is screened for the saturated thickness of the aquifer. The dash (-) indicates sample is available but was not analyzed; an asterisk (*) indicates the sample was not analyzed and is no longer available.]

\begin{tabular}{|c|c|c|c|c|c|c|c|}
\hline \multicolumn{8}{|c|}{ 15-Meter point sampler } \\
\hline \multirow{2}{*}{ Date } & \multirow{2}{*}{ Clock time } & \multirow{2}{*}{$\begin{array}{c}\text { Elapsed } \\
\text { time } \\
\text { (min) }\end{array}$} & \multirow{2}{*}{$\begin{array}{l}\text { Boron } \\
(\mu \mathrm{g} / \mathrm{L})\end{array}$} & \multirow{2}{*}{$\begin{array}{c}\text { Natural } \\
\text { organics } \\
(\mathrm{mg} \mathrm{C} / \mathrm{L})\end{array}$} & \multicolumn{3}{|c|}{$\mathrm{C} / \mathrm{Co} \times 10^{5}$} \\
\hline & & & & & $\mathrm{F}-12$ & $\mathrm{BCF}$ & DBM \\
\hline $5-24-78$ & 1400 & 0 & -- & 0.4 & 6.4 & 1.4 & 0 \\
\hline Do. & 1514 & 74 & 80 & .6 & $*$ & $*$ & $*$ \\
\hline Do. & 1540 & 100 & -- & .6 & $*$ & $*$ & $*$ \\
\hline Do. & 1600 & 120 & -- & -- & 280 & 1.3 & 380 \\
\hline Do. & 1645 & 165 & 80 & .6 & $*$ & $*$ & $*$ \\
\hline Do. & 1700 & 180 & --- & -- & 12 & 3.2 & 1.3 \\
\hline Do. & 1740 & 220 & --- & .7 & $*$ & $*$ & $*$ \\
\hline Do. & 1744 & 224 & 70 & -- & $*$ & $*$ & $*$ \\
\hline Do. & 1858 & 298 & --- & .9 & $*$ & $*$ & $*$ \\
\hline Do. & 2003 & 363 & 80 & .7 & $*$ & $*$ & $*$ \\
\hline Do. & 2102 & 422 & --- & .6 & $*$ & $*$ & $*$ \\
\hline Do. & 2205 & 485 & 70 & -- & $*$ & $*$ & $*$ \\
\hline $5-25-78$ & 1105 & 1,265 & 70 & -- & $*$ & $*$ & $*$ \\
\hline Do. & 1200 & 1,320 & --- & .7 & 6.4 & 1.4 & 0 \\
\hline Do. & 1400 & 1,440 & 73 & -- & $*$ & $*$ & $*$ \\
\hline Do. & 1600 & 1,560 & 371 & --- & $*$ & $*$ & $*$ \\
\hline Do. & 1700 & 1,620 & 70 & -- & $*$ & $*$ & $*$ \\
\hline
\end{tabular}


Table 44.--Cumulative volume of water injected after selected times during the August 1978 injection test

[Injection rate held constant at $5.05 \mathrm{~L} / \mathrm{s}$ throughout the test]

\begin{tabular}{llll}
\hline Date & Time & Cumulative volume & \\
& & $(\mathrm{L})$ & Remarks \\
& & & \\
$8-05-78$ & 1754 & 0 & Injection started. \\
$8-08-78$ & 1200 & $1,201,000$ & Start of first tracer experiment. \\
$8-10-78$ & 1300 & $2,093,000$ & Start of second tracer experiment. \\
$8-12-78$ & 1710 & $3,038,900$ & Start of third tracer experiment. \\
$8-14-78$ & 1200 & $3,817,100$ & Start of fourth tracer experiment. \\
$8-15-78$ & 1000 & $4,216,800$ & Start of fifth tracer experiment. \\
$8-15-78$ & 1935 & $4,390,900$ & End of injection. \\
\hline
\end{tabular}


Vertical well-bore flow-meter data were collected approximately twice daily during tracer injection to determine the zones being recharged. Results of these measurements are shown in figure 18.

Water levels also were measured twice daily in the various piezometers and in selected observation we1ls. These data are too few to analyze for hydraulic properties of the aquifer. Data for such analyses had been collected during the March 1977 pumping test and the March 1978 injection test. August water-level data are not presented here.

\section{Sampling Schedule and Tracer Data}

Data presented in the following sections represent analyses of samples collected during the tests conducted August 8-15, 1978. The tables are arranged chronologically and labeled according to the sampling location, as in the previous sections.

During the first experiment, which began at 1200 on August 8, both bromide $(\mathrm{Br})$ as sodium bromide and boron (B) as boric acid were added to the injection water. Bromide and boron were added simultaneously for two reasons: (1) For a comparison between the two tracers; and (2) known conservative tracers were needed to redefine the flow field, because the well had been redeveloped shortly after the May test to remove entrained air and particulates.

During the second and third experiments, bromide and boron data are provided for comparison with organic tracers used during the experiments. In the fourth experiment, a comparison is made between the following eight tracers: fluoride (F), chloride (C1), bromide ( $B r)$, iodide (I), yeast, Freon-11, Freon-12, and $\mathrm{CBrCl}_{2} \mathrm{~F}$. A fifth experiment was conducted using only the three fluorocarbons Freon-12, Freon-11, and $\mathrm{CBrCl}_{2} \mathrm{~F}$. This experiment was performed because the concentrations of these three compounds in experiment 4 were too small. (Because hydrologic conditions and injection times for experiments 4 and 5 were identical, the data are presented together in tables 75 through 82 in terms of elapsed time.) Data in table 45 summarize information about the tracers used in each of the five experiments. Chemical composition of ground water used as injection water remained virtually constant throughout the test; a typical analysis is given in table 46 .

Concentrations of the inorganic tracers were determined in the laboratory; consequently, many more analyses could be performed than with the halocarbons and several of the nonvolatile organic compounds. Because of greater frequency of inorganic tracer data and because these tracers were used in all four experiments during the August test, these data are given separately in tables 47 to 60 .

For the second and third experiments during the August test, severa1 compounds were added to the water, each with a different initial-input concentration. To compare the concentration of tracer (C) that is observed 


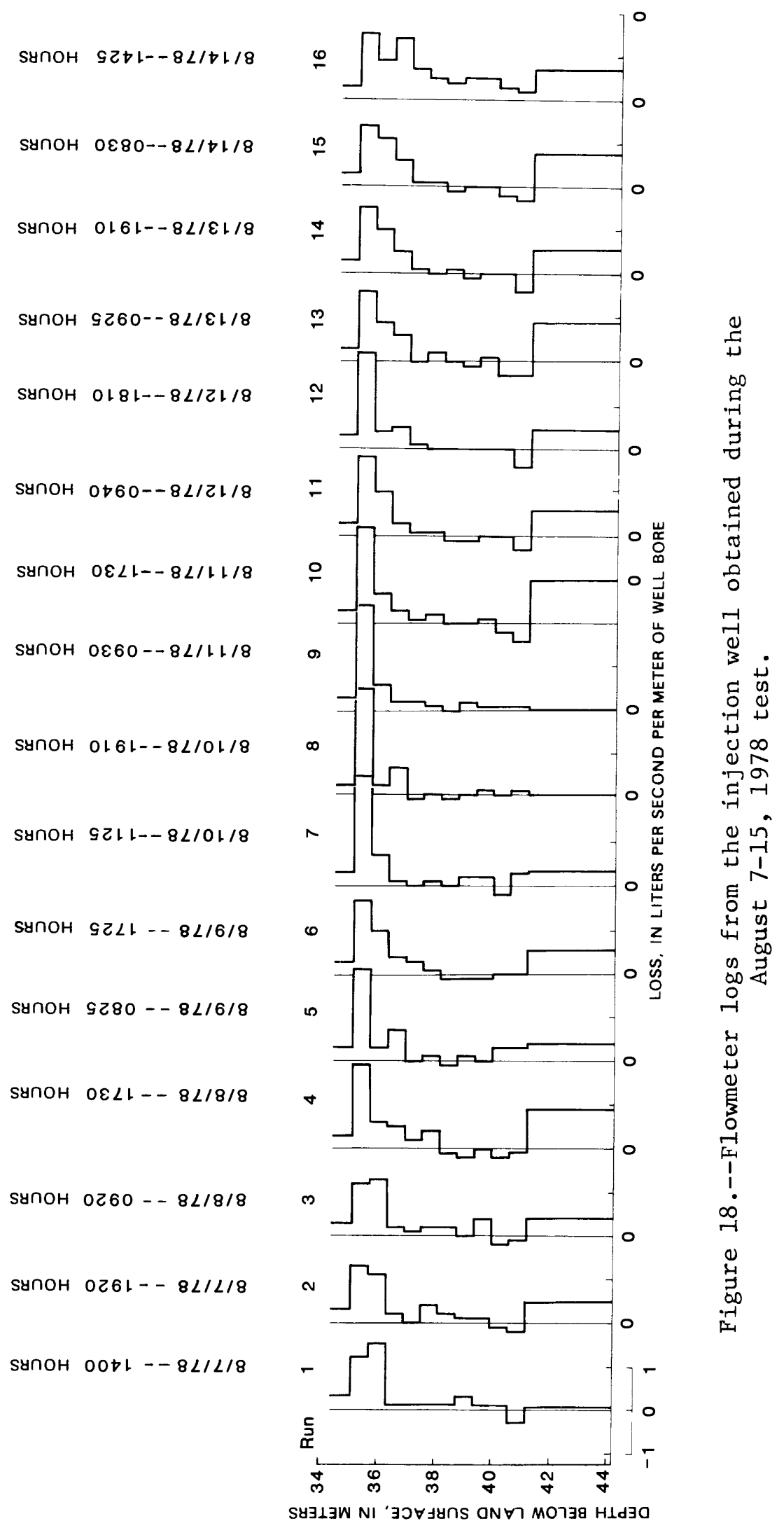


Table 45.--Tracer information for the August 1978 test

\begin{tabular}{|c|c|c|c|c|}
\hline Tracer & $\begin{array}{l}\text { Injection } \\
\text { duration }\end{array}$ & $\begin{array}{l}\text { Start } \\
\text { time }\end{array}$ & Date & $\begin{array}{c}\text { Initial } \\
\text { concentration } \\
(\mathrm{mg} / \mathrm{L})\end{array}$ \\
\hline \multicolumn{5}{|l|}{ First experiment: } \\
\hline Boron------ & $6 \mathrm{~h}$ & 1200 & $8-8-78$ & 3.61 \\
\hline Bromide--- & $6 \mathrm{~h}$ & 1200 & do. & 36.0 \\
\hline Copper----_-_----- & $6 \mathrm{~h}$ & 1200 & do. & 2.0 \\
\hline Deuterium----_--_--- & $6 \mathrm{~h}$ & 1200 & do. & trace \\
\hline $\mathrm{CBrClF}_{2}-\cdots$ & $4.5 \mathrm{~h}$ & 1200 & do. & 3.9 \\
\hline \multicolumn{5}{|l|}{ Second experiment: } \\
\hline Bromide--- & $5 \mathrm{~h}$ & 1300 & $8-10-78$ & 20 \\
\hline Ethanol- & $4 \mathrm{~h}$ & 1300 & do. & 24 \\
\hline Acetate-- & $3 \mathrm{~h}$ & 1300 & do. & 10 \\
\hline Phenolphthalein----- & $4 \mathrm{~h}$ & 1300 & do. & 2 \\
\hline Benzoate----------- & $3 \mathrm{~h}$ & 1300 & do. & 10.4 \\
\hline Freon-12--_- & $9 \mathrm{~h}$ & 1300 & do. & 8.6 \\
\hline \multicolumn{5}{|l|}{ Third experiment: } \\
\hline Boron-- & $6 \mathrm{~h}$ & 1710 & $8-12-78$ & 3.93 \\
\hline Ethanol- & $8 \mathrm{~h}$ & 1710 & do. & 11.6 \\
\hline Aniline-- & $8 \mathrm{~h}$ & 1710 & do. & 9.4 \\
\hline Et hylamine-- - & $8 \mathrm{~h}$ & 1710 & do. & 25 \\
\hline $\mathrm{CBr}_{2} \mathrm{~F}_{2}$ & $14 \mathrm{~h}$ & 1710 & do. & 1.9 \\
\hline \multicolumn{5}{|l|}{ Fourth experiment: } \\
\hline F1uoride--_- & $2 \min$ & 1200 & $8-14-78$ & 96 \\
\hline Chloride---_- & $2 \min$ & 1200 & do. & 1,300 \\
\hline Bromide-----_------ & $2 \mathrm{~min}$ & 1200 & do. & 150 \\
\hline Iodide-_-_-_-_-_-_ & $2 \min$ & 1200 & do. & $60 \quad 1$ \\
\hline Fifth experiment:- & $2 \min$ & 1200 & do. & $1.44 \times 10^{5}$ \\
\hline Freon-11--_-_- & $2 \mathrm{~min}$ & 1000 & $8-15-78$ & 51 \\
\hline Freon-12-_-_- & $2 \mathrm{~min}$ & 1000 & do & 470 \\
\hline $\mathrm{CBrC} 1 \mathrm{~F}_{2}---$ & $2 \mathrm{~min}$ & 1000 & do. & 62 \\
\hline
\end{tabular}

T/ Colonies of yeast per milliliter of injection water.

I Data for the fifth experiment are presented with data for the fourth experiment. 
Table 46.--Analysis of the ground water used during the August 1978 tracer test [Analyses by U.S. Geological Survey Central Laboratory]

\begin{tabular}{|c|c|}
\hline Constituent & $\mathrm{mg} / \mathrm{L}$ \\
\hline Calcium (Ca) & 36 \\
\hline Magnesium (Mg) - & 20 \\
\hline Sodium $(\mathrm{Na})--$ & 26 \\
\hline Potassium (K) & 8.5 \\
\hline Chloride (C1) - & 17 \\
\hline Sulfate $\left(\mathrm{SO}_{4}\right)$ & 26 \\
\hline Alkalinity $\left(\mathrm{CaCO}_{3}\right)-$ & 170 \\
\hline Lithium (Li) & .03 \\
\hline Nitrate $(\mathrm{N})-$ & 1.5 \\
\hline Silica $\left(\mathrm{SiO}_{2}\right)$ & 43 \\
\hline Strontium (Sr) & 1.6 \\
\hline Boron (B) - & .1 \\
\hline Iron $(\mathrm{Fe})$ & .01 \\
\hline Copper $(\mathrm{Cu})-$ & .01 \\
\hline Fluoride (F) - & 3.0 \\
\hline Iodide $(\mathrm{I})$ & .09 \\
\hline Hardness (noncarbonate)-- & 4 \\
\hline Total hardness-_-_-_- & 170 \\
\hline SAR (dimensionless) & .9 \\
\hline $\mathrm{pH}$ (units) - & 7.57 \\
\hline Alkalinity (on-site as $\mathrm{HCO}_{3}^{-}$) - - - & 110 \\
\hline Specific conductance $(\mu \mathrm{mhos} / \mathrm{cm})---$ & 456 \\
\hline
\end{tabular}


Table 47.--Data obtained from the tracer experiments conducted during August 8-15, 1978, at the Stanton, Tex. site

[Samples are from the observation well located $2 \mathrm{~m}$ from the injection well.

The 2-m well is screened for the full-saturated thickness of the aquifer. The dash (-) indicates sample is available but was not analyzed; an asterisk (*) indicates the sample was not analyzed and is no longer available.]

\begin{tabular}{|c|c|c|c|c|c|c|}
\hline \multicolumn{7}{|c|}{ 2-Meter well } \\
\hline Date & Clock time & $\begin{array}{l}\text { Elapsed } \\
\text { (d) }\end{array}$ & $\begin{array}{l}\text { time } \\
\text { (min) }\end{array}$ & $\begin{array}{l}\text { Boron } \\
(\mu \mathrm{g} / \mathrm{L})\end{array}$ & $\begin{array}{c}\text { Bromide } \\
(\mathrm{mg} / \mathrm{L})\end{array}$ & $\begin{array}{l}\mathrm{CBrC} \mathrm{F}_{2} \\
(\mathrm{mg} / \mathrm{L})\end{array}$ \\
\hline $8-8-78$ & 1200 & First tracer & experiment & started & & \\
\hline $8-8-78$ & 1200 & 0 & 0 & 242 & --- & $*$ \\
\hline Do. & 1210 & 0 & 10 & -- & --- & $*$ \\
\hline Do. & 1220 & 0 & 20 & -- & --- & $\star$ \\
\hline Do. & 1230 & 0 & 30 & 231 & --- & 0 \\
\hline Do. & 1240 & 0 & 40 & -- & $--\infty$ & $*$ \\
\hline Do. & 1250 & 0 & 50 & -- & --- & .01 \\
\hline Do. & 1300 & 0 & 60 & 270 & $-\cdots$ & .01 \\
\hline Do. & 1310 & 0 & 70 & -- & --- & $*$ \\
\hline Do. & 1320 & 0 & 80 & -- & --- & $\star$ \\
\hline Do. & 1330 & 0 & 90 & 379 & 1.60 & .03 \\
\hline Do. & 1340 & 0 & 100 & -- & --- & $*$ \\
\hline Do. & 1350 & 0 & 110 & -- & --- & $*$ \\
\hline Do. & 1400 & 0 & 120 & 509 & 3.20 & .11 \\
\hline Do. & 1410 & 0 & 130 & -- & --- & $*$ \\
\hline Do. & 1420 & 0 & 140 & -- & $-\cdots$ & $*$ \\
\hline Do. & 1430 & 0 & 150 & 678 & 4.80 & .10 \\
\hline Do. & 1440 & 0 & 160 & -- & --- & $*$ \\
\hline Do. & 1450 & 0 & 170 & -- & $-\cdots$ & $*$ \\
\hline Do. & 1500 & 0 & 180 & 818 & 7.40 & .40 \\
\hline Do. & 1510 & 0 & 190 & --- & --- & $*$ \\
\hline Do. & 1520 & 0 & 200 & -- & --- & $*$ \\
\hline
\end{tabular}


Table 47.--Data obtained from the tracer experiments conducted during August 8-15, 1978, at the Stanton, Tex. site--Continued

\begin{tabular}{|c|c|c|c|c|c|c|}
\hline \multicolumn{7}{|c|}{ 2-Meter we11 } \\
\hline Date & C1ock time & $\begin{array}{l}\text { Elapsed } \\
\text { (d) }\end{array}$ & $\begin{array}{l}\text { time } \\
\text { (min) }\end{array}$ & $\begin{array}{l}\text { Boron } \\
(\mu \mathrm{g} / \mathrm{L})\end{array}$ & $\begin{array}{c}\text { Bromide } \\
(\mathrm{mg} / \mathrm{L})\end{array}$ & $\begin{array}{c}\mathrm{CBrClF}_{2} \\
(\mathrm{mg} / \mathrm{L})\end{array}$ \\
\hline $8-8-78$ & 1200 & First tracer & exper & t starte & ontinued & \\
\hline $8-8-78$ & 1530 & 0 & 210 & 967 & 9.40 & 0.60 \\
\hline Do. & 1540 & 0 & 220 & - - - & ---- & .68 \\
\hline Do. & 1550 & 0 & 230 & $-\cdots$ & ---- & $*$ \\
\hline Do. & 1600 & 0 & 240 & 1,135 & 10.70 & .09 \\
\hline Do. & 1630 & 0 & 270 & 1,297 & 13.30 & .54 \\
\hline Do. & 1700 & 0 & 300 & 1,448 & 14.60 & .55 \\
\hline Do. & 1730 & 0 & 330 & 1,481 & 14.80 & $*$ \\
\hline Do. & 1800 & 0 & 360 & 1,575 & 16.00 & .09 \\
\hline Do. & 1815 & 0 & 375 & ---- & ---- & $*$ \\
\hline Do. & 1830 & 0 & 390 & 1,635 & 16.90 & $*$ \\
\hline Do. & 1845 & 0 & 405 & ---- & ---- & $*$ \\
\hline Do. & 1900 & 0 & 420 & ----- & ----- & .06 \\
\hline Do. & 1915 & 0 & 435 & 1,704 & 16.90 & $*$ \\
\hline Do. & 1930 & 0 & 450 & ----- & ----- & $*$ \\
\hline Do . & 1945 & 0 & 465 & 1,575 & 15.70 & $*$ \\
\hline Do. & 2000 & 0 & 480 & -- & --- & $*$ \\
\hline Do. & 2020 & 0 & 500 & -- - & ---- & $*$ \\
\hline Do. & 2030 & 0 & 510 & 1,503 & 13.50 & $*$ \\
\hline Do. & 2045 & 0 & 525 & --- & --- & $*$ \\
\hline Do. & 2100 & 0 & 540 & 1,282 & 11.80 & .04 \\
\hline Do. & 2120 & 0 & 560 & ----- & ---- & $*$ \\
\hline Do. & 2140 & 0 & 580 & 1,165 & 9.80 & $*$ \\
\hline Do. & 2200 & 0 & 600 & 1,130 & 9.30 & .42 \\
\hline Do. & 2230 & 0 & 630 & 1,004 & 8.20 & $*$ \\
\hline Do. & 2300 & 0 & 660 & 958 & 6.70 & .03 \\
\hline Do. & 2400 & 0 & 720 & 850 & 6.10 & .14 \\
\hline
\end{tabular}


Table 47.--Data obtained from the tracer experiments conducted during August 8-15, 1978, at the Stanton, Tex. site--Continued

\begin{tabular}{|c|c|c|c|c|c|c|}
\hline \multicolumn{7}{|c|}{ 2-Meter well } \\
\hline Date & Clock time & $\begin{array}{l}\text { Elapsed } \\
\text { (d) }\end{array}$ & $\begin{array}{l}\text { time } \\
\text { (min) }\end{array}$ & $\begin{array}{l}\text { Boron } \\
(\mu \mathrm{g} / \mathrm{L})\end{array}$ & $\begin{array}{c}\text { Bromide } \\
(\mathrm{mg} / \mathrm{L})\end{array}$ & $\begin{array}{l}\mathrm{CBrC} \mathrm{F}_{2} \\
(\mathrm{mg} / \mathrm{L})\end{array}$ \\
\hline $8-8-78$ & 1200 & First tracer & experiment $s$ & started--C & ntinued & \\
\hline $8-9-78$ & 0200 & 0 & 840 & 704 & 4.20 & 0.13 \\
\hline Do. & 0400 & 0 & 960 & 603 & 3.30 & .08 \\
\hline Do. & 0600 & 0 & 1,080 & 497 & 2.70 & * \\
\hline Do. & 0815 & 0 & 1,215 & 444 & 2.30 & $*$ \\
\hline Do. & 1000 & 0 & 1,320 & -- & 1.90 & .02 \\
\hline Do. & 1200 & 1 & 0 & 379 & 1.90 & $*$ \\
\hline Do. & 1415 & 1 & 135 & -- & 1.90 & $*$ \\
\hline Do. & 1600 & 1 & 240 & 340 & 1.80 & $*$ \\
\hline Do. & 1800 & 1 & 360 & -- & 1.70 & $*$ \\
\hline Do. & 2130 & 1 & 570 & 288 & 1.50 & $*$ \\
\hline $8-10-78$ & 0605 & 1 & 1,085 & -- & 1.16 & $*$ \\
\hline Do. & 0610 & 1 & 1,090 & 278 & ---- & $*$ \\
\hline Do. & 0800 & 1 & 1,200 & -- & 1.20 & $*$ \\
\hline Do. & 1000 & 1 & 1,320 & 248 & 1.15 & $*$ \\
\hline Do. & 1158 & 1 & 1,438 & 244 & 1.10 & $*$ \\
\hline $8-10-78$ & 1300 & Second trace & ex experiment & th started & & \\
\hline $8-10-78$ & 1300 & 2 & 60 & 244 & .6 & $*$ \\
\hline Do. & 1310 & 2 & 70 & -- & ---- & $*$ \\
\hline Do. & 1320 & 2 & 80 & -- & ---- & $*$ \\
\hline Do. & 1330 & 2 & 90 & -- & .6 & $*$ \\
\hline Do. & 1340 & 2 & 100 & -- & ---- & $*$ \\
\hline Do. & 1350 & 2 & 110 & -- & ---- & $*$ \\
\hline Do. & 1400 & 2 & 120 & 237 & .8 & $*$ \\
\hline Do. & 1410 & 2 & 130 & --- & --- & $*$ \\
\hline
\end{tabular}


Table 47.--Data obtained from the tracer experiments conducted during August 8-15, 1978, at the Stanton, Tex. site--Continued

\begin{tabular}{|c|c|c|c|c|c|c|}
\hline \multicolumn{7}{|c|}{ 2-Meter we11 } \\
\hline Date & Clock time & $\begin{array}{l}\text { Elapsed } \\
\text { (d) }\end{array}$ & $\begin{array}{l}\text { time } \\
\text { (min) }\end{array}$ & $\begin{array}{l}\text { Boron } \\
(\mu \mathrm{g} / \mathrm{L})\end{array}$ & $\begin{array}{c}\text { Bromide } \\
(\mathrm{mg} / \mathrm{L})\end{array}$ & $\begin{array}{l}\mathrm{CBrClF}_{2} \\
(\mathrm{mg} / \mathrm{L})\end{array}$ \\
\hline $8-10-78$ & 1300 & Second tracer & experiment & starte & ontinued & \\
\hline $8-10-78$ & 1420 & 2 & 140 & --- & --- & $*$ \\
\hline Do. & 1430 & 2 & 150 & -- & 1.3 & $*$ \\
\hline Do. & 1445 & 2 & 165 & -- & --- & $*$ \\
\hline Do. & 1500 & 2 & 180 & --- & 2.5 & $*$ \\
\hline Do. & 1515 & 2 & 195 & --- & --- & $*$ \\
\hline Do. & 1530 & 2 & 210 & --- & 3.8 & $*$ \\
\hline Do. & 1545 & 2 & 225 & -- & 4.8 & $*$ \\
\hline Do. & 1600 & 2 & 240 & 231 & --- & $*$ \\
\hline Do . & 1630 & 2 & 270 & -- & 4.8 & $*$ \\
\hline Do. & 1700 & 2 & 300 & -- & 5.2 & $*$ \\
\hline Do. & 1730 & 2 & 330 & -- & 5.0 & $*$ \\
\hline Do. & 1800 & 2 & 360 & -- & 4.6 & $*$ \\
\hline Do. & 1900 & 2 & 420 & --- & 4.2 & $*$ \\
\hline Do. & 2000 & 2 & 480 & 220 & 4.8 & $*$ \\
\hline Do. & 2010 & 2 & 490 & -- & --- & $*$ \\
\hline Do. & 2100 & 2 & 540 & --- & 4.4 & $*$ \\
\hline Do. & 2210 & 2 & 610 & -- & 2.8 & $*$ \\
\hline Do. & 2215 & 2 & 615 & -- & --- & $*$ \\
\hline Do. & 2310 & 2 & 670 & --- & 2.6 & $*$ \\
\hline Do. & 2320 & 2 & 680 & -- & -- & $*$ \\
\hline Do. & 2400 & 2 & 720 & 211 & 2.1 & $*$ \\
\hline $8-11-78$ & 0030 & 2 & 750 & --- & -- & $*$ \\
\hline Do. & 0100 & 2 & 780 & -- & -- & $*$ \\
\hline Do. & 0205 & 2 & 845 & --- & 1.5 & $*$ \\
\hline Do. & 0225 & 2 & 865 & -- & --- & $*$ \\
\hline
\end{tabular}


Table 47.--Data obtained from the tracer experiments conducted during August 8-15, 1978, at the Stanton, Tex. site--Continued

\begin{tabular}{|c|c|c|c|c|c|c|}
\hline \multicolumn{7}{|c|}{ 2-Meter wel1 } \\
\hline Date & Clock time & $\begin{array}{l}\text { Elapsed } \\
\text { (d) }\end{array}$ & $\begin{array}{l}\text { time } \\
\text { (min) }\end{array}$ & $\begin{array}{l}\text { Boron } \\
(\mu g / L)\end{array}$ & $\begin{array}{r}\text { Bromide } \\
(\mathrm{mg} / \mathrm{L})\end{array}$ & $\begin{array}{c}\mathrm{CBrClF}_{2} \\
(\mathrm{mg} / \mathrm{L})\end{array}$ \\
\hline $8-11-78$ & 1300 & Second tracer & experiment & started & ontinued & \\
\hline $8-11-78$ & 0300 & 2 & 900 & --- & -- & $*$ \\
\hline Do. & 0305 & 2 & 905 & -- & --- & $*$ \\
\hline Do. & 0315 & 2 & 915 & -- & -- & $*$ \\
\hline Do. & 0405 & 2 & 965 & -- & 1.3 & $*$ \\
\hline Do. & 0600 & 2 & 1,080 & 198 & 1.8 & $*$ \\
\hline Do. & 0800 & 2 & 1,200 & -- & 1.8 & $*$ \\
\hline Do. & 1010 & 2 & 1,330 & -- & 1.5 & $*$ \\
\hline Do. & 1020 & 2 & 1,340 & -- & -- & $*$ \\
\hline Do. & 1200 & 3 & 0 & 181 & 1.4 & $*$ \\
\hline Do. & 1213 & 3 & 13 & -- & -- & $*$ \\
\hline Do. & 1400 & 3 & 60 & -- & 1.3 & $*$ \\
\hline Do. & 1408 & 3 & 68 & -- & -- & $*$ \\
\hline Do. & 1555 & 3 & 235 & -- & --- & $*$ \\
\hline Do. & 1805 & 3 & 365 & 179 & 1.2 & $*$ \\
\hline Do. & 2100 & 3 & 540 & -- & 1.1 & $*$ \\
\hline Do. & 2400 & 3 & 720 & 181 & 1.0 & $\star$ \\
\hline $8-12-78$ & 0600 & 3 & 1,080 & -- & .9 & $*$ \\
\hline Do. & 1043 & 3 & 1,363 & 163 & .9 & $*$ \\
\hline Do. & 1650 & 4 & 290 & -- & -- & $*$ \\
\hline Do. & 1700 & 4 & 300 & 152 & .9 & $*$ \\
\hline $8-12-78$ & 1710 & Third tracer & experiment & started & & \\
\hline $8-12-78$ & 1713 & 4 & 313 & -- & .9 & $*$ \\
\hline Do. & 1800 & 4 & 360 & 402 & -- & $*$ \\
\hline Do. & 1815 & 4 & 375 & 416 & -- & $*$ \\
\hline
\end{tabular}


Table 47.--Data obtained from the tracer experiments conducted during August 8-15, 1978, at the Stanton, Tex. site-Continued

\begin{tabular}{|c|c|c|c|c|c|c|}
\hline \multicolumn{7}{|c|}{ 2-Meter wel1 } \\
\hline Date & Clock time & $\begin{array}{l}\text { Elapsed } \\
\text { (d) }\end{array}$ & $\begin{array}{l}\text { time } \\
\text { (min) }\end{array}$ & $\begin{array}{l}\text { Boron } \\
(\mu g / L)\end{array}$ & $\begin{array}{c}\text { Bromide } \\
(\mathrm{mg} / \mathrm{L})\end{array}$ & $\begin{array}{l}\mathrm{CBrC} \mathrm{F}_{2} \\
(\mathrm{mg} / \mathrm{L})\end{array}$ \\
\hline $8-12-78$ & 1710 & Third tracer & experiment & started & ntinued & \\
\hline $8-12-78$ & 1830 & 4 & 390 & 396 & -- & $*$ \\
\hline Do. & 1845 & 4 & 405 & 396 & -- & $*$ \\
\hline Do. & 1900 & 4 & 420 & 515 & -- & $*$ \\
\hline Do. & 1915 & 4 & 435 & 475 & -- & $*$ \\
\hline Do . & 1930 & 4 & 450 & 574 & -- & $*$ \\
\hline Do. & 1945 & 4 & 465 & 851 & -- & $*$ \\
\hline Do. & 2000 & 4 & 480 & 1,030 & 0.9 & $*$ \\
\hline Do. & 2030 & 4 & 510 & ---- & -- & $*$ \\
\hline Do. & 2100 & 4 & 540 & ---- & -- & $*$ \\
\hline Do. & 2130 & 4 & 570 & 1,235 & -- & $*$ \\
\hline Do. & 2200 & 4 & 600 & --- & -- & $*$ \\
\hline Do. & 2230 & 4 & 630 & 1,268 & -- & $*$ \\
\hline Do . & 2300 & 4 & 660 & 1,475 & .9 & $*$ \\
\hline Do. & 2330 & 4 & 690 & 1,525 & -- & $*$ \\
\hline Do. & 2400 & 4 & 720 & 1,628 & --- & $*$ \\
\hline $8-13-78$ & 0100 & 4 & 780 & 1,485 & --- & $*$ \\
\hline Do. & 0130 & 4 & 810 & ---- & -- & $*$ \\
\hline Do. & 0200 & 4 & 840 & $-\cdots-$ & -- & $*$ \\
\hline Do. & 0230 & 4 & 870 & 1,210 & -- & $*$ \\
\hline Do. & 0300 & 4 & 900 & ---- & --- & $*$ \\
\hline Do. & 0330 & 4 & 930 & ---- & -- & $*$ \\
\hline Do. & 0400 & 4 & 960 & 994 & -- & $*$ \\
\hline Do. & 0500 & 4 & 1,020 & ---- & --- & $*$ \\
\hline Do. & 0600 & 4 & 1,080 & 793 & .9 & $*$ \\
\hline Do. & 0700 & 4 & 1,140 & ---- & --- & $*$ \\
\hline Do. & 0800 & 4 & 1,200 & 651 & --- & $*$ \\
\hline Do. & 0900 & 4 & 1,260 & ---- & -- & $*$ \\
\hline
\end{tabular}


Table 47.--Data obtained from the tracer experiments conducted during August 8-15, 1978, at the Stanton, Tex. site--Continued

\begin{tabular}{|c|c|c|c|c|c|c|}
\hline \multicolumn{7}{|c|}{ 2-Meter we11 } \\
\hline Date & Clock time & $\begin{array}{l}\text { Elapsed } \\
\text { (d) }\end{array}$ & $\begin{array}{l}\text { time } \\
\text { (min) }\end{array}$ & $\begin{array}{l}\text { Boron } \\
(\mu \mathrm{g} / \mathrm{L})\end{array}$ & $\begin{array}{c}\text { Bromide } \\
(\mathrm{mg} / \mathrm{L})\end{array}$ & $\begin{array}{c}\mathrm{CBrClF}_{2} \\
(\mathrm{mg} / \mathrm{L})\end{array}$ \\
\hline $8-12-78$ & 1710 & Third tracer & experiment & started-- & ntinued & \\
\hline $8-13-78$ & 0900 & 4 & 1,260 & -- & -- & $*$ \\
\hline Do. & 1000 & 4 & 1,320 & 573 & --- & $*$ \\
\hline Do. & 1200 & 5 & 0 & --- & 0.9 & $*$ \\
\hline Do. & 1400 & 5 & 120 & 453 & -- & $*$ \\
\hline Do. & 1600 & 5 & 240 & 398 & -- & $*$ \\
\hline Do. & 2000 & 5 & 480 & 347 & --- & * \\
\hline Do. & 2300 & 5 & 540 & 296 & .9 & $*$ \\
\hline $8-14-78$ & 1200 & Fourth tracer & experiment & started & & \\
\hline $8-14-78$ & 1200 & 6 & 0 & 230 & .8 & $*$ \\
\hline Do. & 1215 & 6 & 15 & -- & -- & $*$ \\
\hline Do. & 1230 & 6 & 30 & 230 & 1.8 & * \\
\hline Do. & 1245 & 6 & 45 & -- & -- & $*$ \\
\hline Do. & 1300 & 6 & 60 & 210 & 4.5 & $*$ \\
\hline Do. & 1315 & 6 & 75 & -- & --- & $*$ \\
\hline Do. & 1330 & 6 & 90 & -- & --- & $*$ \\
\hline Do. & 1345 & 6 & 105 & 240 & 8.0 & $*$ \\
\hline Do. & 1400 & 6 & 120 & 240 & 10.0 & $*$ \\
\hline Do. & 1430 & 6 & 150 & 220 & 12.0 & $*$ \\
\hline Do. & 1500 & 6 & 180 & 230 & 4.5 & $*$ \\
\hline Do. & 1530 & 6 & 210 & --- & --- & $*$ \\
\hline Do. & 1600 & 6 & 240 & 230 & 3.8 & $*$ \\
\hline Do. & 1630 & 6 & 270 & --- & -- & $*$ \\
\hline Do. & 1700 & 6 & 300 & 240 & 3.4 & $*$ \\
\hline Do. & 1730 & 6 & 330 & -- & - & $*$ \\
\hline
\end{tabular}


Table 47.--Data obtained from the tracer experiments conducted during August 8-15, 1978, at the Stanton, Tex. site--Continued

\begin{tabular}{|c|c|c|c|c|c|c|}
\hline \multicolumn{7}{|c|}{ 2-Meter we11 } \\
\hline Date & Clock time & $\begin{array}{l}\text { Elapsed } \\
\text { (d) }\end{array}$ & $\begin{array}{l}\text { time } \\
\text { (min) }\end{array}$ & $\begin{array}{l}\text { Boron } \\
(\mu \mathrm{g} / \mathrm{L})\end{array}$ & $\begin{array}{c}\text { Bromide } \\
(\mathrm{mg} / \mathrm{L})\end{array}$ & $\begin{array}{c}\mathrm{CBrClF}_{2} \\
(\mathrm{mg} / \mathrm{L})\end{array}$ \\
\hline $8-14-78$ & 1200 & Fourth tracer & experiment & starte & ontinued & \\
\hline $8-14-78$ & 1800 & 6 & 360 & 220 & --- & $*$ \\
\hline Do. & 1900 & 6 & 420 & -- & --- & $*$ \\
\hline Do. & 2000 & 6 & 480 & -- & --- & $*$ \\
\hline Do. & 2100 & 6 & 540 & -- & --- & $*$ \\
\hline Do. & 2200 & 6 & 600 & -- & --- & $*$ \\
\hline Do. & 2300 & 6 & 660 & -- & $\cdots$ & $*$ \\
\hline Do. & 2400 & 6 & 720 & 210 & 0.86 & $*$ \\
\hline $8-15-78$ & 0200 & 6 & 840 & -- & .70 & $*$ \\
\hline Do. & 0400 & 6 & 960 & -- & --- & $*$ \\
\hline Do. & 0600 & 6 & 1,080 & -- & --- & $*$ \\
\hline Do. & 0800 & 6 & 1,200 & -- & --- & $*$ \\
\hline Do. & 1000 & 6 & 1,320 & 190 & .43 & $*$ \\
\hline Do. & 1200 & 7 & 0 & -- & --- & $*$ \\
\hline Do. & 1400 & 7 & 120 & -- & --- & $*$ \\
\hline Do. & 1800 & 7 & 360 & 190 & $-\cdots$ & $*$ \\
\hline
\end{tabular}


Table 48.--Data obtained from the tracer experiments conducted during August. 8-75, 2978, at the Stanton, Tex. site

[Samples are from the point sampler designated 2-m black located $41.6 \mathrm{~m}$ below land surface and $2 \mathrm{~m}$ radially from the injection well. The dash (-) indicates sample is available but was not analyzed; an asterisk (*) indicates the sample was not analyzed and is no longer available.]

\begin{tabular}{|c|c|c|c|c|c|c|c|}
\hline \multicolumn{8}{|c|}{ 2-Meter black point sampler } \\
\hline Date & Clock time & & $\begin{array}{l}\text { Elapsed } \\
\text { (d) }\end{array}$ & $\begin{array}{l}\text { time } \\
\text { (min) }\end{array}$ & $\begin{array}{l}\text { Boron } \\
(\mu g / L)\end{array}$ & $\begin{array}{c}\text { Bromide } \\
(\mathrm{mg} / \mathrm{L})\end{array}$ & $\begin{array}{c}\mathrm{CBrClF}_{2} \\
(\mathrm{mg} / \mathrm{L})\end{array}$ \\
\hline $8-8-78$ & 1200 & First & tracer & experiment & started & & \\
\hline $8-8-78$ & 1200 & & 0 & 0 & 222 & 1.8 & $\star$ \\
\hline Do. & 1210 & & 0 & 10 & -- & --- & $\stackrel{4}{*}$ \\
\hline Do. & 1220 & & 0 & 20 & --- & --- & 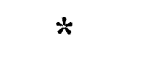 \\
\hline Do. & 1230 & & 0 & 30 & -- & --- & * \\
\hline Do. & 1240 & & 0 & 40 & --- & -- & $*$ \\
\hline Do. & 1250 & & 0 & 50 & -- & --- & * \\
\hline Do. & 1300 & & 0 & 60 & 191 & .9 & $*$ \\
\hline Do. & 1310 & & 0 & 70 & -- & -- & $\frac{1}{x}$ \\
\hline Do. & 1320 & & 0 & 80 & -- & -- & $*$ \\
\hline Do. & 1330 & & 0 & 90 & --- & -- & $*$ \\
\hline Do. & 1340 & & 0 & 100 & --- & -- & $*$ \\
\hline Do. & 1350 & & 0 & 110 & -- & -- & * \\
\hline Do. & 1400 & & 0 & 120 & -- & -- & $\stackrel{t}{*}$ \\
\hline Do. & 1407 & & 0 & 127 & 187 & .9 & $*$ \\
\hline Do. & 1410 & & 0 & 130 & -- & -- & $*$ \\
\hline Do. & 1420 & & 0 & 140 & -- & --- & $*$ \\
\hline Do. & 1430 & & 0 & 150 & --- & -- & $*$ \\
\hline Do. & 1440 & & 0 & 160 & -- & -- & $\stackrel{4}{*}$ \\
\hline Do. & 1450 & & 0 & 170 & --- & -- & * \\
\hline Do. & 1500 & & 0 & 180 & 196 & 2.2 & 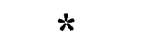 \\
\hline Do. & 1510 & & 0 & 190 & --- & --- & $*$ \\
\hline
\end{tabular}


Table 48.--Data obtained from the tracer experiment conducted during August 8-15, 1978, at the Stanton, Tex. site--Continued

\begin{tabular}{|c|c|c|c|c|c|c|c|}
\hline \multicolumn{8}{|c|}{ 2-Meter black point sampler } \\
\hline Date & Clock time & & $\begin{array}{l}\text { Elapsed } \\
\text { (d) }\end{array}$ & $\begin{array}{l}\text { time } \\
\text { (min) }\end{array}$ & $\begin{array}{l}\text { Boron } \\
(\mu \mathrm{g} / \mathrm{L})\end{array}$ & $\begin{array}{c}\text { Bromide } \\
(\mathrm{mg} / \mathrm{L})\end{array}$ & $\begin{array}{c}\mathrm{CBrClF}_{2} \\
(\mathrm{mg} / \mathrm{L})\end{array}$ \\
\hline $8-8-78$ & 1200 & First & tracer & experiment & started & ntinued & \\
\hline $8-8-78$ & 1520 & & 0 & 200 & -- & -- & * \\
\hline Do. & 1530 & & 0 & 210 & --- & -- & $*$ \\
\hline Do. & 1540 & & 0 & 220 & -- & -- & $*$ \\
\hline Do. & 1550 & & 0 & 230 & -- & --- & $*$ \\
\hline Do. & 1600 & & 0 & 240 & 187 & 0.9 & $*$ \\
\hline Do. & 1630 & & 0 & 270 & --- & -- & $*$ \\
\hline Do. & 1700 & & 0 & 300 & 187 & .8 & $*$ \\
\hline Do. & 1730 & & 0 & 330 & -- & --- & $*$ \\
\hline Do. & 1800 & & 0 & 360 & 189 & 1.0 & $*$ \\
\hline Do. & 1815 & & 0 & 375 & -- & -- & $*$ \\
\hline Do. & 1830 & & 0 & 390 & -- & --- & $*$ \\
\hline Do. & 1845 & & 0 & 405 & -- & --- & $*$ \\
\hline Do. & 1900 & & 0 & 420 & 183 & .9 & $*$ \\
\hline Do. & 1915 & & 0 & 435 & -- & -- & $*$ \\
\hline Do. & 1930 & & 0 & 450 & -- & $\cdots$ & $*$ \\
\hline Do. & 1945 & & 0 & 465 & -- & --- & $*$ \\
\hline Do. & 2000 & & 0 & 480 & 187 & .9 & $*$ \\
\hline Do. & 2020 & & 0 & 500 & --- & $\cdots$ & $*$ \\
\hline Do. & 2030 & & 0 & 510 & -- & -- & $*$ \\
\hline Do. & 2045 & & 0 & 525 & --- & -- & $*$ \\
\hline Do. & 2100 & & 0 & 540 & 191 & -- & $*$ \\
\hline Do. & 2120 & & 0 & 560 & --- & --- & $*$ \\
\hline Do. & 2140 & & 0 & 580 & --- & -- & $*$ \\
\hline Do. & 2200 & & 0 & 600 & 178 & .8 & $*$ \\
\hline Do. & 2230 & & 0 & 630 & -- & --- & $*$ \\
\hline
\end{tabular}


Table 48.--Data obtained from the tracer experiments conducted during August 8-15, 1978, at the Stanton, Tex. site--Continued

\begin{tabular}{|c|c|c|c|c|c|c|}
\hline & & 2-Meter b & olack point & sampler & & \\
\hline Date & Clock time & $\begin{array}{l}\text { Elapsed } \\
\text { (d) }\end{array}$ & $\begin{array}{l}\text { time } \\
\text { (min) }\end{array}$ & $\begin{array}{l}\text { Boron } \\
(\mu g / L)\end{array}$ & $\begin{array}{r}\text { Bromide } \\
(\mathrm{mg} / \mathrm{L})\end{array}$ & $\begin{array}{l}\mathrm{CBrClF}_{2} \\
(\mathrm{mg} / \mathrm{L})\end{array}$ \\
\hline $8-8-78$ & 1200 & First tracer & experiment & started--Co & ntinued & \\
\hline $8-8-78$ & 2300 & 0 & 660 & --- & -- & $*$ \\
\hline Do. & 2400 & 0 & 720 & 177 & 1.0 & * \\
\hline $8-9-78$ & 0200 & 0 & 840 & 181 & --- & * \\
\hline Do. & 0400 & 0 & 960 & 179 & .9 & $*$ \\
\hline Do. & 0600 & 0 & 1,080 & 189 & 1.1 & $*$ \\
\hline Do. & 0815 & 0 & 1,215 & 250 & 1.4 & $*$ \\
\hline Do. & 1000 & 0 & 1,320 & 235 & 1.6 & $*$ \\
\hline Do. & 1200 & 1 & 0 & 245 & 2.2 & $*$ \\
\hline Do. & 1415 & 1 & 1,350 & 243 & 2.8 & $*$ \\
\hline Do. & 1600 & 1 & 240 & 264 & 3.5 & * \\
\hline Do & 1800 & 1 & 360 & 273 & 3.9 & $*$ \\
\hline $8-10-78$ & 0605 & 1 & 1,085 & 343 & 6.2 & $*$ \\
\hline Do. & 0800 & 1 & 1,200 & 372 & 6.4 & $*$ \\
\hline $8-10-78$ & 1300 & Second tracer & : experiment & initiated & & \\
\hline $8-10-78$ & 1300 & 2 & 60 & -- & -- & $*$ \\
\hline Do. & 1310 & 2 & 70 & 405 & 5.5 & $*$ \\
\hline Do. & 1400 & 2 & 120 & 412 & 5.5 & $*$ \\
\hline Do. & 1600 & 2 & 240 & -- & 4.1 & $*$ \\
\hline Do. & 1650 & 2 & 290 & 398 & -- & $*$ \\
\hline Do. & 2000 & 2 & 480 & 392 & 4.2 & * \\
\hline Do. & 2400 & 2 & 720 & 376 & 3.8 & $*$ \\
\hline $8-11-78$ & 0600 & 2 & 1,080 & 365 & 4.4 & * \\
\hline Do. & 1200 & 3 & 0 & 359 & 4.2 & $*$ \\
\hline Do. & 1800 & 3 & 360 & 346 & 4.2 & $*$ \\
\hline Do. & 2100 & 3 & 540 & 321 & 3.8 & $*$ \\
\hline
\end{tabular}


Table 48.--Data obtained from the tracer experiments conducted during August 8-15, 1978, at the Stanton, Tex. site--Continued

\begin{tabular}{|c|c|c|c|c|c|c|}
\hline \multicolumn{7}{|c|}{ 2-Meter black point sampler } \\
\hline Date & Clock time & $\begin{array}{l}\text { Elapsed } \\
\text { (d) }\end{array}$ & $\begin{array}{l}\text { time } \\
\text { (min) }\end{array}$ & $\begin{array}{l}\text { Boron } \\
(\mu \mathrm{g} / \mathrm{L})\end{array}$ & $\begin{array}{c}\text { Bromide } \\
(\mathrm{mg} / \mathrm{L})\end{array}$ & $\begin{array}{c}\mathrm{CBrClF}_{2} \\
(\mathrm{mg} / \mathrm{L})\end{array}$ \\
\hline $8-10-78$ & 1300 & Second tracer & experiment & started- & ontinued & \\
\hline $8-12-78$ & 0600 & 3 & 1,080 & 301 & 4.2 & $*$ \\
\hline Do. & 1035 & 3 & 1,355 & 288 & 4.4 & $*$ \\
\hline Do. & 1200 & 4 & 0 & --- & --- & $*$ \\
\hline Do. & 1650 & 4 & 290 & 284 & 4.9 & * \\
\hline Do. & 1700 & 4 & 300 & --- & --- & $*$ \\
\hline $8-12-78$ & 1710 & Third tracer & experiment & started & & \\
\hline $8-12-78$ & 2000 & 4 & 480 & 262 & 3.7 & $*$ \\
\hline Do. & 2400 & 4 & 720 & 253 & 4.1 & $*$ \\
\hline $8-13-78$ & 0100 & 4 & 780 & 264 & 4.5 & $*$ \\
\hline Do. & 0500 & 4 & 1,020 & 257 & 4.0 & $*$ \\
\hline Do. & 0900 & 4 & 1,260 & 279 & 4.6 & $*$ \\
\hline Do. & 1200 & 5 & 0 & 264 & 3.7 & $*$ \\
\hline Do. & 1400 & 5 & 120 & 279 & 4.4 & $*$ \\
\hline Do. & 1800 & 5 & 360 & 293 & 3.1 & $*$ \\
\hline Do. & 2300 & 5 & 660 & 324 & 4.7 & $*$ \\
\hline $8-14-78$ & 0600 & 5 & 1,080 & 348 & 4.6 & $*$ \\
\hline $8-14-78$ & 1200 & Fourth tracer & experiment & $=$ started & & \\
\hline $8-14-78$ & 1200 & 6 & 0 & 350 & 1.2 & $*$ \\
\hline Do. & 1215 & 6 & 15 & --- & --- & $*$ \\
\hline Do. & 1230 & 6 & 30 & --- & ---- & $*$ \\
\hline Do. & 1245 & 6 & 45 & --- & ---- & $*$ \\
\hline Do. & 1300 & 6 & 60 & 360 & .76 & $*$ \\
\hline Do. & 1315 & 6 & 75 & --- & --- & 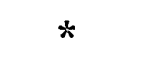 \\
\hline
\end{tabular}


Table 48.--Data obtained from the tracer experiments conducted during August 8-15, 1978 at the Stanton, Tex. site--Continued

\begin{tabular}{|c|c|c|c|c|c|c|}
\hline \multicolumn{7}{|c|}{ 2-Meter black point sampler } \\
\hline Date & Clock time & $\begin{array}{l}\text { Elapsed } \\
\text { (d) }\end{array}$ & $\begin{array}{l}\text { time } \\
\text { (min) }\end{array}$ & $\begin{array}{l}\text { Boron } \\
(\mu \mathrm{g} / \mathrm{L})\end{array}$ & $\begin{array}{c}\text { Bromide } \\
(\mathrm{mg} / \mathrm{L})\end{array}$ & $\begin{array}{l}\mathrm{CBrClF}_{2} \\
(\mathrm{mg} / \mathrm{L})\end{array}$ \\
\hline $8-14-78$ & 1200 & Fourth tracer & experiment & started & ontinued & \\
\hline $8-14-78$ & 1330 & 6 & 90 & --- & --- & $*$ \\
\hline Do. & 1345 & 6 & 105 & --- & --- & $*$ \\
\hline Do. & 1400 & 6 & 120 & -- & ---- & $*$ \\
\hline Do. & 1430 & 6 & 150 & -- & ---- & $*$ \\
\hline Do & 1500 & 6 & 180 & --- & ---- & $*$ \\
\hline Do. & 1530 & 6 & 210 & --- & ---- & $*$ \\
\hline Do. & 1600 & 6 & 240 & -- & --- & $*$ \\
\hline Do. & 1630 & 6 & 270 & --- & --- & $*$ \\
\hline Do. & 1700 & 6 & 300 & --- & ---- & $*$ \\
\hline Do. & 1730 & 6 & 330 & -- & --- & $*$ \\
\hline Do. & 1800 & 6 & 360 & 370 & 1.1 & $*$ \\
\hline Do. & 1900 & 6 & 420 & --- & --- & $*$ \\
\hline Do. & 2000 & 6 & 480 & -- & --- & $*$ \\
\hline Do. & 2100 & 6 & 540 & --- & --- & $*$ \\
\hline Do. & 2200 & 6 & 600 & 380 & .6 & $\star$ \\
\hline Do. & 2300 & 6 & 660 & $-\cdots$ & --- & $*$ \\
\hline Do. & 2400 & 6 & 720 & --- & --- & $*$ \\
\hline $8-15-78$ & 0200 & 6 & 840 & 370 & 1.0 & $*$ \\
\hline Do. & 0400 & 6 & 960 & --- & --- & $*$ \\
\hline Do. & 0600 & 6 & 1,080 & 380 & 1.4 & $*$ \\
\hline Do. & 0800 & 6 & 1,200 & --- & --- & $*$ \\
\hline Do. & 1000 & 6 & 1,320 & 370 & .78 & $*$ \\
\hline Do. & 1200 & 7 & 0 & -- & ---- & $*$ \\
\hline Do. & 1400 & 7 & 120 & --- & ---- & $*$ \\
\hline Do. & 1800 & 7 & 360 & 380 & ---- & $*$ \\
\hline
\end{tabular}


Table 49.--Data obtained from the tracer experiments conducted during August 8-15, 1978, at the Stanton, Tex. site

[Samples are from the point sampler designated 2-m red located $2 \mathrm{~m}$ radially from the injection well and $38.7 \mathrm{~m}$ below land surface. The dash (-) indicates sample is available but was not analyzed; an asterisk (*) indicates the sample was not analyzed and is no longer available.]

\begin{tabular}{|c|c|c|c|c|c|c|c|}
\hline \multicolumn{8}{|c|}{ 2-Meter red point sampler } \\
\hline Date & Clock time & & $\begin{array}{l}\text { Elapsed } \\
\text { (d) }\end{array}$ & $\begin{array}{l}\text { time } \\
\text { (min) }\end{array}$ & $\begin{array}{l}\text { Boron } \\
(\mu g / L)\end{array}$ & $\begin{array}{c}\text { Bromide } \\
(\mathrm{mg} / \mathrm{L})\end{array}$ & $\begin{array}{c}\mathrm{CBrClF}_{2} \\
(\mathrm{mg} / \mathrm{L})\end{array}$ \\
\hline $8-8-78$ & 1200 & First & tracer & experiment & started & & \\
\hline $8-8-78$ & 1200 & & 0 & 0 & 101 & --- & $*$ \\
\hline Do. & 1210 & & 0 & 10 & -- & 1.0 & $*$ \\
\hline Do. & 1220 & & 0 & 20 & --- & ---- & 0 \\
\hline Do. & 1230 & & 0 & 30 & 107 & --- & 0 \\
\hline Do. & 1240 & & 0 & 40 & -- & ---- & 0 \\
\hline Do. & 1250 & & 0 & 50 & --- & --- & 0 \\
\hline Do. & 1300 & & 0 & 60 & 101 & ---- & 0 \\
\hline Do. & 1310 & & 0 & 70 & --- & .8 & $\star$ \\
\hline Do. & 1320 & & 0 & 80 & --- & ---- & $*$ \\
\hline Do. & 1330 & & 0 & 90 & 113 & ---- & .01 \\
\hline Do. & 1340 & & 0 & 100 & --- & 1.8 & $*$ \\
\hline Do. & 1350 & & 0 & 110 & -- & ---- & $*$ \\
\hline Do. & 1400 & & 0 & 120 & 169 & --- & $*$ \\
\hline Do. & 1410 & & 0 & 130 & --- & 2.0 & .01 \\
\hline Do. & 1420 & & 0 & 140 & -- & --- & $*$ \\
\hline Do. & 1430 & & 0 & 150 & 227 & 2.30 & .02 \\
\hline Do. & 1440 & & 0 & 160 & --- & --- & $*$ \\
\hline Do. & 1450 & & 0 & 170 & -- & ---- & $*$ \\
\hline Do. & 1500 & & 0 & 180 & 333 & 3.70 & .04 \\
\hline Do. & 1510 & & 0 & 190 & --- & ---- & $*$ \\
\hline Do. & 1520 & & 0 & 200 & 487 & 5.4 & $*$ \\
\hline Do. & 1530 & & 0 & 210 & --- & ---- & $*$ \\
\hline
\end{tabular}


Table 49.--Data obtained from the tracer experiments conducted during August 8-15, 1978, at the Stanton, Tex. site--Continued

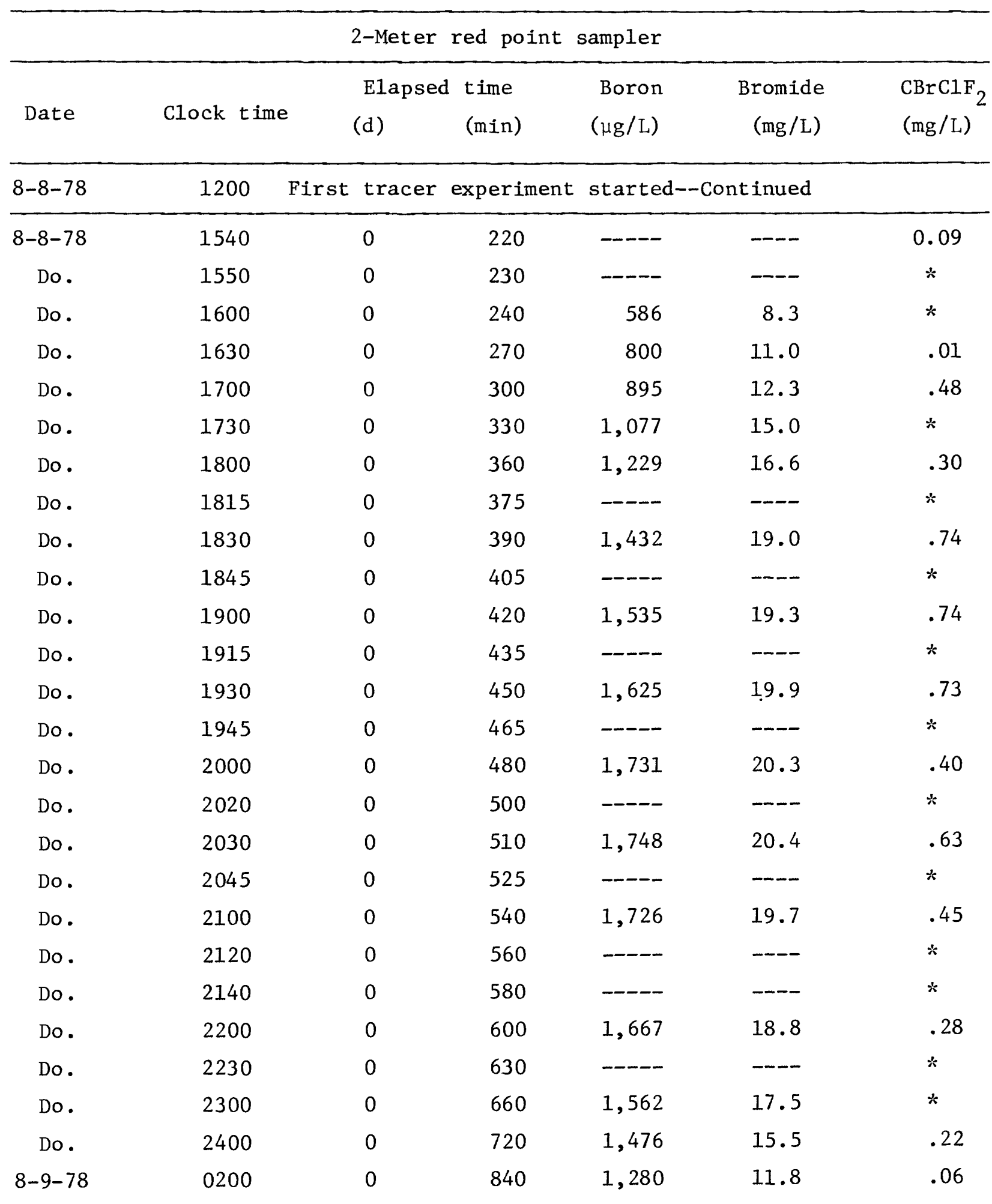


Table 49.--Data obtained from the tracer experiments conducted during August 8-15, 1978, at the Stanton, Tex. site--Continued

\begin{tabular}{|c|c|c|c|c|c|c|}
\hline \multicolumn{7}{|c|}{ 2-Meter red point sampler } \\
\hline Date & Clock time & $\begin{array}{l}\text { Elapsed } \\
\text { (d) }\end{array}$ & $\begin{array}{l}\text { time } \\
\text { (min) }\end{array}$ & $\begin{array}{l}\text { Boron } \\
(\mu \mathrm{g} / \mathrm{L})\end{array}$ & $\begin{array}{c}\text { Bromide } \\
(\mathrm{mg} / \mathrm{L})\end{array}$ & $\begin{array}{l}\mathrm{CBrC1F}_{2} \\
(\mathrm{mg} / \mathrm{L})\end{array}$ \\
\hline $8-8-78$ & 1200 & First tracer & experiment & started-- & ntinued & \\
\hline $8-9-78$ & 0400 & 0 & 960 & 968 & 8.9 & 0.13 \\
\hline Do. & 0600 & 0 & 1,080 & 747 & 6.4 & $*$ \\
\hline Do. & 0815 & 0 & 1,115 & 613 & 5.4 & $*$ \\
\hline Do. & 1000 & 0 & 1,320 & -- & 3.8 & $*$ \\
\hline Do. & 1200 & 1 & 0 & 440 & 3.6 & $*$ \\
\hline Do. & 1415 & 1 & 135 & -- & 1.7 & $*$ \\
\hline Do. & 1600 & 1 & 240 & 308 & 2.0 & $*$ \\
\hline Do. & 1800 & 1 & 360 & -- & 1.7 & $*$ \\
\hline Do. & 2130 & 1 & 570 & 251 & 1.8 & .12 \\
\hline $8-10-78$ & 0605 & 1 & 1,085 & -- & 1.2 & $*$ \\
\hline Do & 0800 & 1 & 1,200 & -- & 1.2 & $*$ \\
\hline Do. & 1000 & 1 & 1,320 & 179 & -- & $*$ \\
\hline $8-10-78$ & 1300 & Second tracer & experiment & started & & \\
\hline $8-10-78$ & 1320 & 2 & 80 & 159 & -- & $*$ \\
\hline Do. & 1330 & 2 & 90 & -- & 3.0 & $*$ \\
\hline Do. & 1340 & 2 & 100 & -- & --- & $*$ \\
\hline Do. & 1350 & 2 & 110 & $-\cdots$ & --- & $*$ \\
\hline Do. & 1400 & 2 & 120 & 165 & -- & $*$ \\
\hline Do. & 1410 & 2 & 130 & -- & 3.1 & $*$ \\
\hline Do. & 1420 & 2 & 140 & $-\cdots$ & -- & $\star$ \\
\hline Do. & 1430 & 2 & 150 & -- & 2.6 & $*$ \\
\hline Do. & 1445 & 2 & 165 & -- & -- & $*$ \\
\hline Do. & 1500 & 2 & 180 & -- & 3.6 & $*$ \\
\hline Do. & 1515 & 2 & 195 & --- & --- & $*$ \\
\hline
\end{tabular}


Table 49.--Data obtained from the tracer experiments conducted during August 8-15, 1978, at the Stanton, Tex. site--Continued

\begin{tabular}{|c|c|c|c|c|c|c|}
\hline \multicolumn{7}{|c|}{ 2-Meter red point sampler } \\
\hline Date & Clock time & $\begin{array}{l}\text { Elapsed } \\
\text { (d) }\end{array}$ & $\begin{array}{l}\text { time } \\
\text { (min) }\end{array}$ & $\begin{array}{l}\text { Boron } \\
(\mu \mathrm{g} / \mathrm{L})\end{array}$ & $\begin{array}{c}\text { Bromide } \\
(\mathrm{mg} / \mathrm{L})\end{array}$ & $\begin{array}{l}\mathrm{CBrClF}_{2} \\
(\mathrm{mg} / \mathrm{L})\end{array}$ \\
\hline $8-10-78$ & 1300 & Second tracer & experiment & starte & ontinued & \\
\hline $8-10-78$ & 1530 & 2 & 210 & -- & 3.1 & $*$ \\
\hline Do. & 1545 & 2 & 225 & --- & --- & $*$ \\
\hline Do. & 1600 & 2 & 240 & 157 & --- & $\doteqdot$ \\
\hline Do. & 1630 & 2 & 270 & -- & --- & $*$ \\
\hline Do. & 1700 & 2 & 300 & -- & 4.4 & $*$ \\
\hline Do. & 1730 & 2 & 330 & -- & 6.2 & $*$ \\
\hline Do. & 1800 & 2 & 360 & 148 & --- & * \\
\hline Do. & 1900 & 2 & 420 & -- & 9.5 & $\star$ \\
\hline Do. & 2000 & 2 & 480 & -- & $-\cdots$ & $*$ \\
\hline Do. & 2100 & 2 & 540 & -- & 14.2 & $*$ \\
\hline Do. & 2200 & 2 & 600 & -- & 14.0 & $*$ \\
\hline Do. & 2300 & 2 & 660 & -- & 12.2 & $*$ \\
\hline Do. & 2400 & 2 & 720 & 134 & --- & $*$ \\
\hline $8-11-78$ & 0100 & 2 & 780 & -- & $-\cdots$ & * \\
\hline Do. & 0204 & 2 & 844 & -- & 9.4 & $*$ \\
\hline Do. & 0300 & 2 & 900 & -- & 9.0 & $*$ \\
\hline Do. & 0600 & 2 & 1,080 & 132 & --- & $*$ \\
\hline Do. & 0800 & 2 & 1,200 & -- & 5.6 & $*$ \\
\hline Do. & 1005 & 2 & 1,325 & -- & 6.1 & $*$ \\
\hline Do. & 1200 & 3 & 0 & 120 & -- & $*$ \\
\hline Do. & 1400 & 3 & 120 & -- & 5.8 & $*$ \\
\hline Do. & 1800 & 3 & 360 & 120 & $\cdots$ & $*$ \\
\hline Do. & 2100 & 3 & 540 & -- & 4.8 & $*$ \\
\hline $8-12-78$ & 0200 & 3 & 840 & -- & --- & $*$ \\
\hline
\end{tabular}


Table 49.--Data obtained from the tracer experiments conducted during August 8-15, 1978, at the Stanton, Tex. site--Continued

\begin{tabular}{|c|c|c|c|c|c|c|}
\hline \multicolumn{7}{|c|}{ 2-Meter red point sampler } \\
\hline Date & Clock time & $\begin{array}{l}\text { Elapsed } \\
\text { (d) }\end{array}$ & $\begin{array}{l}\text { time } \\
\text { (min) }\end{array}$ & $\begin{array}{l}\text { Boron } \\
(\mu g / L)\end{array}$ & $\begin{array}{c}\text { Bromide } \\
(\mathrm{mg} / \mathrm{L})\end{array}$ & $\begin{array}{l}\mathrm{CBrClF}_{2} \\
(\mathrm{mg} / \mathrm{L})\end{array}$ \\
\hline $8-10-78$ & 1300 & Second tracer & experiment & t starte & ontinued & \\
\hline $8-12-78$ & 1035 & 3 & 1,355 & 118 & --- & $*$ \\
\hline Do. & 1650 & 4 & 290 & --- & 1.7 & $*$ \\
\hline $8-12-78$ & 1710 & Third tracer & experiment & started & & \\
\hline $8-12-78$ & 1730 & 4 & 330 & 120 & --- & $*$ \\
\hline Do. & 1800 & 4 & 360 & 118 & --- & $*$ \\
\hline Do. & 1815 & 4 & 375 & --- & 1.6 & $*$ \\
\hline Do. & 1830 & 4 & 390 & 120 & --- & $*$ \\
\hline Do. & 1845 & 4 & 405 & --- & 1.6 & $*$ \\
\hline Do. & 1900 & 4 & 420 & 118 & --- & 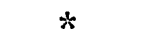 \\
\hline Do. & 1915 & 4 & 435 & --- & 1.4 & $*$ \\
\hline Do. & 1930 & 4 & 450 & --- & 1.1 & $*$ \\
\hline Do. & 1945 & 4 & 465 & --- & 1.7 & $*$ \\
\hline Do. & 2000 & 4 & 480 & 167 & --- & $*$ \\
\hline Do. & 2030 & 4 & 510 & --- & 1.2 & $*$ \\
\hline Do. & 2100 & 4 & 540 & 242 & --- & $*$ \\
\hline Do. & 2130 & 4 & 570 & --- & 1.5 & $*$ \\
\hline Do. & 2200 & 4 & 600 & 291 & --- & $*$ \\
\hline Do. & 2230 & 4 & 630 & --- & 1.1 & $*$ \\
\hline Do. & 2300 & 4 & 660 & 510 & --- & $*$ \\
\hline Do. & 2400 & 4 & 720 & 710 & 1.5 & $*$ \\
\hline $8-13-78$ & 0030 & 4 & 750 & 870 & --- & $*$ \\
\hline Do. & 0100 & 4 & 780 & --- & 1.2 & $*$ \\
\hline Do. & 0130 & 4 & 810 & 1200 & 1.2 & $*$ \\
\hline Do. & 0200 & 4 & 840 & --- & 1.4 & $*$ \\
\hline Do. & 0230 & 4 & 870 & --- & 1.5 & $*$ \\
\hline
\end{tabular}


Table 49.--Data obtained from the tracer experiments conducted during August 8-15, 1978, at the Stanton, Tex. site--Continued

\begin{tabular}{|c|c|c|c|c|c|c|}
\hline & & 2-Meter & red point $s$ & sampler & & \\
\hline Date & Clock time & $\begin{array}{l}\text { Elapsed } \\
\text { (d) }\end{array}$ & $\begin{array}{l}\text { time } \\
\text { (min) }\end{array}$ & $\begin{array}{l}\text { Boron } \\
(\mu \mathrm{g} / \mathrm{L})\end{array}$ & $\begin{array}{r}\text { Bromide } \\
(\mathrm{mg} / \mathrm{L})\end{array}$ & $\begin{array}{c}\mathrm{CBrClF}_{2} \\
(\mathrm{mg} / \mathrm{L})\end{array}$ \\
\hline $8-12-78$ & 1710 & Third tracer & experiment & started-- & ntinued & \\
\hline $8-13-78$ & 0300 & 4 & 900 & --- & 1.5 & $*$ \\
\hline Do. & 0330 & 4 & 930 & -- & 1.5 & $*$ \\
\hline Do. & 0400 & 4 & 960 & 1570 & -- & $*$ \\
\hline Do. & 0500 & 4 & 1,020 & 1525 & -- & $*$ \\
\hline Do. & 0600 & 4 & 1,080 & -- & 1.6 & $*$ \\
\hline Do. & 0700 & 4 & 1,140 & 1390 & 1.8 & * \\
\hline Do. & 0800 & 4 & 1,200 & 1195 & 1.8 & $*$ \\
\hline Do. & 0900 & 4 & 1,260 & --- & 1.8 & $*$ \\
\hline Do. & 1000 & 4 & 1,320 & 1020 & --- & $*$ \\
\hline Do. & 1200 & 5 & 0 & 920 & 2.1 & $*$ \\
\hline Do. & 1400 & 5 & 120 & 960 & 2.3 & $*$ \\
\hline Do. & 1600 & 5 & 240 & 800 & 2.1 & $*$ \\
\hline Do. & 1800 & 5 & 360 & 510 & 1.8 & $*$ \\
\hline Do. & 2010 & 5 & 490 & -- & 1.8 & $*$ \\
\hline Do. & 2300 & 5 & 660 & -- & 1.9 & $*$ \\
\hline $8-14-78$ & 0300 & 5 & 900 & 472 & -- & $*$ \\
\hline Do. & 0600 & 5 & 1,080 & 329 & -- & $*$ \\
\hline $8-12-78$ & 1200 & Fourth tracer & experiment & started & & \\
\hline $8-12-78$ & 1200 & 6 & 0 & 290 & .1 & $*$ \\
\hline Do. & 1215 & 6 & 15 & --- & -- & $*$ \\
\hline Do. & 1230 & 6 & 30 & 250 & .0 & $*$ \\
\hline Do. & 1245 & 6 & 45 & -- & -- & $*$ \\
\hline Do. & 1300 & 6 & 60 & 230 & .2 & $*$ \\
\hline Do. & 1315 & 6 & 75 & --- & -- & $*$ \\
\hline Do. & 1330 & 6 & 90 & 230 & .8 & $*$ \\
\hline Do. & 1345 & 6 & 105 & --- & --- & $*$ \\
\hline
\end{tabular}


Table 49.--Data obtained from the tracer experiments conducted during August 8-15, 1978, at the Stanton, Tex. site--Continued

\begin{tabular}{|c|c|c|c|c|c|c|}
\hline \multicolumn{7}{|c|}{ 2-Meter red point sampler } \\
\hline Date & Clock time & $\begin{array}{l}\text { Elapsed } \\
\text { (d) }\end{array}$ & $\begin{array}{l}\text { time } \\
\text { (min) }\end{array}$ & $\begin{array}{l}\text { Boron } \\
(\mu \mathrm{g} / \mathrm{L})\end{array}$ & $\begin{array}{r}\text { Bromide } \\
(\mathrm{mg} / \mathrm{L})\end{array}$ & $\begin{array}{l}\mathrm{CBrClF}_{2} \\
(\mathrm{mg} / \mathrm{L})\end{array}$ \\
\hline $8-12-78$ & 1200 & Fourth tracer & experiment & starte & ontinued & \\
\hline $8-12-78$ & 1400 & 6 & 120 & 230 & 1.3 & $*$ \\
\hline Do. & 1430 & 6 & 150 & --- & -- & $*$ \\
\hline Do. & 1500 & 6 & 180 & 230 & 2.3 & $\star$ \\
\hline Do. & 1530 & 6 & 210 & -- & -- & $*$ \\
\hline Do. & 1600 & 6 & 240 & 210 & 3.5 & * \\
\hline Do. & 1630 & 6 & 270 & -- & -- & $*$ \\
\hline Do. & 1700 & 6 & 300 & 230 & 5.0 & $*$ \\
\hline Do. & 1730 & 6 & 330 & -- & $\cdots$ & $*$ \\
\hline Do. & 1800 & 6 & 360 & --- & -- & $*$ \\
\hline Do. & 1900 & 6 & 420 & $\cdots$ & $\cdots$ & $*$ \\
\hline Do. & 2000 & 6 & 480 & -- & -- & $*$ \\
\hline $8-14-78$ & 2100 & 6 & 540 & -- & -- & $*$ \\
\hline Do. & 2200 & 6 & 600 & 190 & 4.4 & $*$ \\
\hline Do. & 2300 & 6 & 660 & -- & --- & * \\
\hline Do. & 2400 & 6 & 720 & 180 & 2.5 & $*$ \\
\hline $8-15-78$ & 0200 & 6 & 840 & 180 & 2.8 & $*$ \\
\hline Do. & 0400 & 6 & 960 & $-\cdots$ & -- & $*$ \\
\hline Do. & 0600 & 6 & 1,080 & 180 & 1.8 & * \\
\hline Do. & 0800 & 6 & 1,200 & $-\cdots$ & -- & $*$ \\
\hline Do. & 1000 & 6 & 1,320 & $-\cdots$ & -- & $*$ \\
\hline Do. & 1200 & 7 & 0 & 160 & -- & $*$ \\
\hline Do. & 1400 & 7 & 120 & 150 & -- & $*$ \\
\hline Do. & 1800 & 7 & 360 & -- & $-\infty$ & $*$ \\
\hline
\end{tabular}


Table 50.--Data obtained from the tracer experiments conducted during August 8-15, 1978, at the Stanton, Tex. site

[Samples are from the point sampler designated 2-m orange located $34.8 \mathrm{~m}$ below land surface and $2 \mathrm{~m}$ radially from the injection well. The dash (-) indicates sample is available but was not analyzed; an asterisk $(*)$ indicates this sample was not analyzed and is no longer available.]

\begin{tabular}{|c|c|c|c|c|c|c|}
\hline \multicolumn{7}{|c|}{ 2-Meter orange point sampler } \\
\hline Date & Clock time & $\begin{array}{l}\text { Elapsed } \\
\text { (d) }\end{array}$ & $\begin{array}{l}\text { time } \\
\text { (min) }\end{array}$ & $\begin{array}{l}\text { Boron } \\
(\mu g / L)\end{array}$ & $\begin{array}{c}\text { Bromide } \\
(\mathrm{mg} / \mathrm{L})\end{array}$ & $\begin{array}{c}\mathrm{CBrC} \mathrm{F}_{2} \\
(\mathrm{mg} / \mathrm{L})\end{array}$ \\
\hline $8-8-78$ & 1200 & \multicolumn{5}{|c|}{ First tracer experiment started } \\
\hline $8-8-78$ & 1200 & 0 & 0 & $-\cdots$ & --- & $*$ \\
\hline Do. & 1215 & 0 & 15 & 173 & 0.8 & $*$ \\
\hline Do. & 1220 & 0 & 20 & ---- & --- & $*$ \\
\hline Do. & 1230 & 0 & 30 & 162 & .8 & $*$ \\
\hline Do. & 1240 & 0 & 40 & ---- & --- & $*$ \\
\hline Do. & 1250 & 0 & 50 & ---- & --- & $*$ \\
\hline Do & 1300 & 0 & 60 & 579 & 3.5 & $*$ \\
\hline Do & 1310 & 0 & 70 & ---- & --- & $*$ \\
\hline Do. & 1320 & 0 & 80 & --- & $-\cdots$ & $*$ \\
\hline Do. & 1330 & 0 & 90 & 1,064 & 9.0 & $*$ \\
\hline Do. & 1340 & 0 & 100 & ---- & --- & $*$ \\
\hline Do. & 1350 & 0 & 110 & --- & $-\cdots$ & $*$ \\
\hline Do. & 1355 & 0 & 115 & --- & --- & $*$ \\
\hline Do. & 1407 & 0 & 127 & 1,640 & 12.5 & $*$ \\
\hline Do. & 1420 & 0 & 140 & ---- & --- & $*$ \\
\hline Do. & 1430 & 0 & 150 & 1,767 & 16.2 & $*$ \\
\hline Do. & 1440 & 0 & 160 & ---- & --- & $*$ \\
\hline Do. & 1450 & 0 & 170 & ---- & --- & $*$ \\
\hline Do. & 1500 & 0 & 180 & 1,973 & 18.2 & $*$ \\
\hline Do. & 1510 & 0 & 190 & ---- & ---- & $*$ \\
\hline
\end{tabular}


Table 50.--Data obtained from the tracer experiment conducted during August 8-15, 1978, at the Stanton, Tex. site--Continued

\begin{tabular}{|c|c|c|c|c|c|c|}
\hline \multicolumn{7}{|c|}{ 2-Meter orange point sampler } \\
\hline Date & Clock time & $\begin{array}{l}\text { Elapsed } \\
\text { (d) }\end{array}$ & $\begin{array}{l}\text { time } \\
\text { (min) }\end{array}$ & $\begin{array}{l}\text { Boron } \\
(\mu \mathrm{g} / \mathrm{L})\end{array}$ & $\begin{array}{c}\text { Bromide } \\
(\mathrm{mg} / \mathrm{L})\end{array}$ & $\begin{array}{c}\mathrm{CBrClF}_{2} \\
(\mathrm{mg} / \mathrm{L})\end{array}$ \\
\hline $8-8-78$ & 1200 & First tracer & experiment & started & ntinued & \\
\hline $8-8-78$ & 1520 & 0 & 200 & --- & ---- & $*$ \\
\hline Do. & 1530 & 0 & 210 & ---- & --- & $*$ \\
\hline Do. & 1540 & 0 & 220 & 2,186 & 19.0 & $*$ \\
\hline Do. & 1550 & 0 & 230 & ---- & ---- & $*$ \\
\hline Do. & 1600 & 0 & 240 & ---- & --- & $*$ \\
\hline Do. & 1630 & 0 & 270 & 2,310 & 21.6 & $*$ \\
\hline Do. & 1700 & 0 & 300 & 2,446 & 21.8 & $*$ \\
\hline Do. & 1730 & 0 & 330 & 2,684 & 21.7 & $*$ \\
\hline Do. & 1800 & 0 & 360 & 2,518 & 24.0 & $*$ \\
\hline Do. & 1815 & 0 & 375 & --- & ---- & $*$ \\
\hline Do. & 1830 & 0 & 390 & 2,596 & 23.1 & $*$ \\
\hline Do. & 1845 & 0 & 405 & ---- & --- & $*$ \\
\hline Do. & 1900 & 0 & 420 & 2,248 & 21.0 & $*$ \\
\hline Do. & 1915 & 0 & 435 & --- & --- & 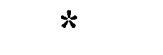 \\
\hline Do. & 1930 & 0 & 450 & 1,878 & 16.0 & 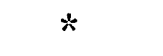 \\
\hline Do. & 1945 & 0 & 465 & -- & --- & $*$ \\
\hline Do. & 2000 & 0 & 480 & 1,481 & 11.8 & $*$ \\
\hline Do. & 2020 & 0 & 500 & -- & ---- & $*$ \\
\hline Do. & 2030 & 0 & 510 & 1,278 & 9.5 & $\star$ \\
\hline Do. & 2045 & 0 & 525 & --- & --- & $*$ \\
\hline Do. & 2100 & 0 & 540 & 1,084 & 8.5 & $*$ \\
\hline Do. & 2120 & 0 & 560 & --- & --- & $*$ \\
\hline Do. & 2140 & 0 & 580 & --- & --- & $*$ \\
\hline Do. & 2200 & 0 & 600 & 886 & 5.3 & $*$ \\
\hline
\end{tabular}


Table 50.--Data obtained from the tracer experiments conducted during August 8-15, 1978, at the Stanton, Tex. site--Continued

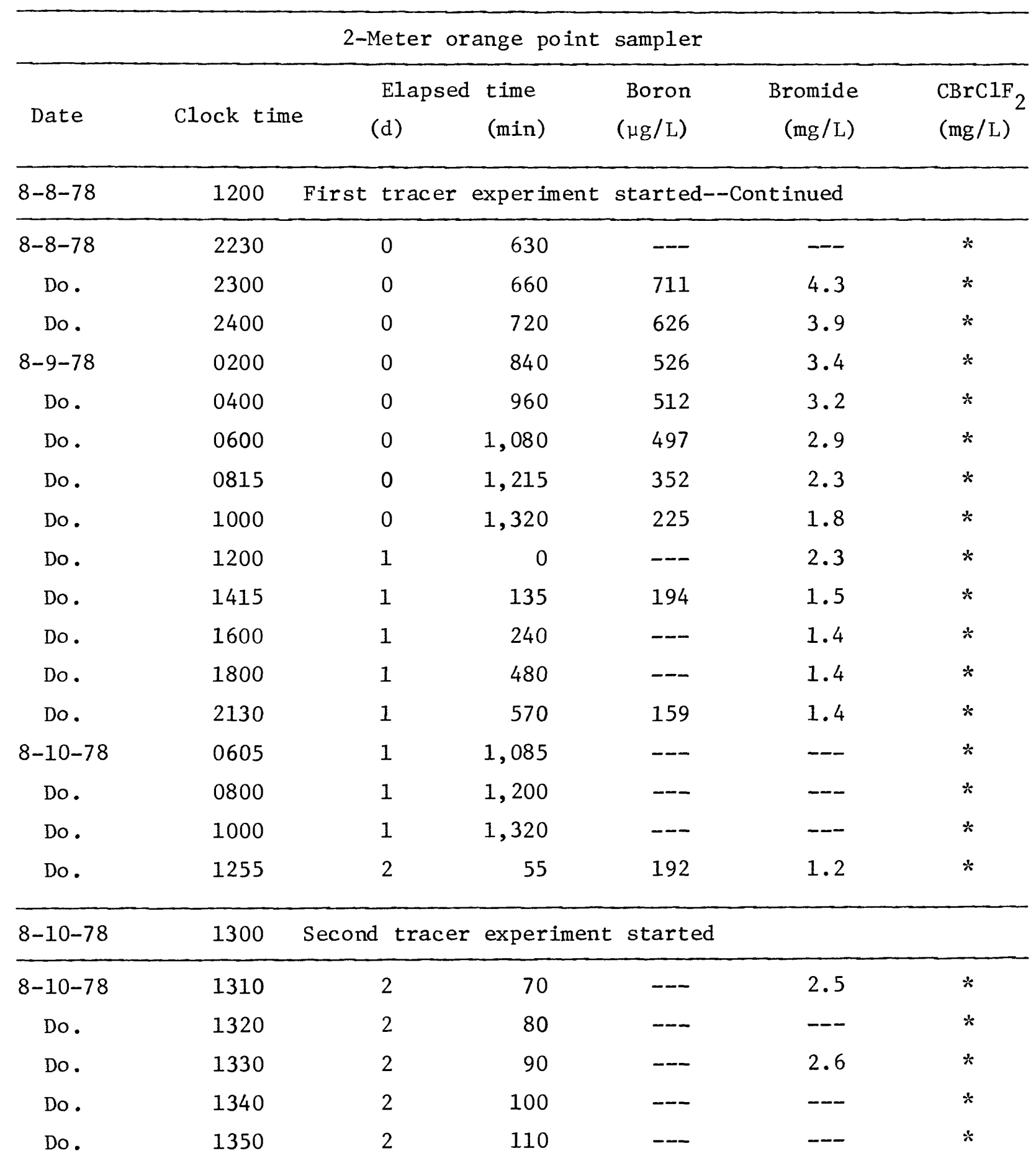


Table 50.--Data obtained from the tracer experiments conducted during August 8-15, 1978, at the Stanton, Tex. site--Continued

\begin{tabular}{|c|c|c|c|c|c|c|}
\hline \multicolumn{7}{|c|}{ 2-Meter orange point sampler } \\
\hline Date & Clock time & $\begin{array}{l}\text { Elapsed } \\
\text { (d) }\end{array}$ & $\begin{array}{l}\text { time } \\
\text { (min) }\end{array}$ & $\begin{array}{l}\text { Boron } \\
(\mu g / L)\end{array}$ & $\begin{array}{c}\text { Bromide } \\
(\mathrm{mg} / \mathrm{L})\end{array}$ & $\begin{array}{l}\mathrm{CBrClF}_{2} \\
(\mathrm{mg} / \mathrm{L})\end{array}$ \\
\hline $8-10-78$ & 1300 & Second tracer & experiment & started & ontinued & \\
\hline $8-10-78$ & 1400 & 2 & 120 & --- & 5.4 & $*$ \\
\hline Do. & 1410 & 2 & 130 & --- & --- & $*$ \\
\hline Do. & 1420 & 2 & 140 & --- & ---- & $*$ \\
\hline Do . & 1430 & 2 & 150 & --- & 12.0 & $*$ \\
\hline Do. & 1445 & 2 & 165 & --- & ---- & $*$ \\
\hline Do. & 1500 & 2 & 180 & --- & 14.2 & $*$ \\
\hline Do. & 1515 & 2 & 195 & --- & ---- & $*$ \\
\hline Do. & 1530 & 2 & 210 & --- & 9.1 & $*$ \\
\hline Do. & 1545 & 2 & 225 & --- & ---- & $*$ \\
\hline Do. & 1600 & 2 & 240 & 161 & 13.8 & $*$ \\
\hline Do. & 1630 & 2 & 270 & -- & 13.8 & $*$ \\
\hline Do. & 1700 & 2 & 300 & --- & 18.4 & $*$ \\
\hline Do. & 1730 & 2 & 330 & --- & 20.2 & 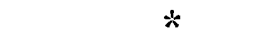 \\
\hline Do. & 1800 & 2 & 360 & --- & 19.0 & $*$ \\
\hline Do. & 1900 & 2 & 420 & 161 & 14.8 & $*$ \\
\hline Do. & 2000 & 2 & 480 & --- & 12.8 & $*$ \\
\hline Do. & 2100 & 2 & 540 & -- & 8.0 & $*$ \\
\hline Do. & 2200 & 2 & 600 & -- & --- & $*$ \\
\hline Do. & 2300 & 2 & 660 & 148 & 4.9 & $*$ \\
\hline Do. & 2400 & 2 & 720 & --- & ---- & $*$ \\
\hline $8-11-78$ & 0100 & 2 & 780 & --- & 5.1 & $*$ \\
\hline Do. & 0300 & 2 & 900 & --- & ---- & $*$ \\
\hline Do. & 0400 & 2 & 960 & --- & ---- & $*$ \\
\hline
\end{tabular}


Table 50.--Data obtained from the tracer experiments conducted during August 8-15, 1978, at the Stanton, Tex. site--Continued

\begin{tabular}{|c|c|c|c|c|c|c|}
\hline \multicolumn{7}{|c|}{ 2-Meter orange point sampler } \\
\hline Date & Clock time & $\begin{array}{l}\text { Elapsed } \\
\text { (d) }\end{array}$ & $\begin{array}{l}\text { time } \\
\text { (min) }\end{array}$ & $\begin{array}{l}\text { Boron } \\
(\mu \mathrm{g} / \mathrm{L})\end{array}$ & $\begin{array}{r}\text { Bromide } \\
(\mathrm{mg} / \mathrm{L})\end{array}$ & $\begin{array}{l}\mathrm{CBrClF}_{2} \\
(\mathrm{mg} / \mathrm{L})\end{array}$ \\
\hline $8-10-78$ & 1300 & Second tracer & experiment & starte & ontinued & \\
\hline $8-11-78$ & 0600 & 2 & 1,080 & 140 & 2.7 & $*$ \\
\hline Do. & 0800 & 2 & 1,200 & ---- & --- & $*$ \\
\hline Do. & 1200 & 3 & 0 & 144 & 2.7 & $*$ \\
\hline Do. & 1410 & 3 & 130 & ---- & --- & $*$ \\
\hline Do. & 1600 & 3 & 240 & ---- & -- & $*$ \\
\hline Do. & 1800 & 3 & 360 & --- & --- & $*$ \\
\hline Do. & 2100 & 3 & 540 & 146 & 2.0 & $*$ \\
\hline $8-12-78$ & 0600 & 3 & 1,080 & --- & -- & $*$ \\
\hline Do. & 1215 & 4 & 15 & ---- & --- & $*$ \\
\hline Do. & 1230 & 4 & 30 & 157 & --- & $*$ \\
\hline $8-12-78$ & 1710 & Third tracer & experiment & started & & \\
\hline $8-12-78$ & 1800 & 4 & 360 & ---- & --- & $*$ \\
\hline Do. & 1815 & 4 & 375 & ---- & -- & $*$ \\
\hline Do. & 1830 & 4 & 390 & 807 & 1.3 & $*$ \\
\hline Do. & 1845 & 4 & 405 & --- & -- & $*$ \\
\hline Do. & 1900 & 4 & 420 & 1,220 & -- & $*$ \\
\hline Do. & 1915 & 4 & 435 & ---- & 2.4 & $*$ \\
\hline Do. & 1930 & 4 & 450 & 1,435 & --- & $*$ \\
\hline Do. & 2030 & 4 & 510 & 1,670 & 2.3 & $*$ \\
\hline Do. & 2100 & 4 & 540 & 1,930 & -- & * \\
\hline Do. & 2130 & 4 & 570 & ---- & 1.6 & $*$ \\
\hline Do. & 2200 & 4 & 600 & 2,055 & -- & $*$ \\
\hline Do. & 2230 & 4 & 630 & --- & --- & $*$ \\
\hline Do. & 2300 & 4 & 660 & 2,165 & 1.7 & $*$ \\
\hline
\end{tabular}


Table 50.--Data obtained from the tracer experiments conducted during August 8-15, 1978, at the Stanton, Tex. site--Continued

\begin{tabular}{|c|c|c|c|c|c|c|}
\hline \multicolumn{7}{|c|}{ 2-Meter orange point sampler } \\
\hline Date & Clock time & $\begin{array}{l}\text { Elapsed } \\
\text { (d) }\end{array}$ & $\begin{array}{l}\text { time } \\
\text { (min) }\end{array}$ & $\begin{array}{l}\text { Boron } \\
(\mu \mathrm{g} / \mathrm{L})\end{array}$ & $\begin{array}{c}\text { Bromide } \\
(\mathrm{mg} / \mathrm{L})\end{array}$ & $\begin{array}{l}\mathrm{CBrClF}_{2} \\
(\mathrm{mg} / \mathrm{L})\end{array}$ \\
\hline $8-12-78$ & 1710 & Third tracer & experiment & started & ntinued & \\
\hline $8-12-78$ & 2330 & 4 & 690 & ---- & --- & $*$ \\
\hline Do. & 2400 & 4 & 720 & ---- & -- & $*$ \\
\hline $8-13-78$ & 0030 & 4 & 750 & 2,005 & --- & $*$ \\
\hline Do. & 0100 & 4 & 780 & ---- & 1.3 & $*$ \\
\hline Do. & 0130 & 4 & 810 & ---- & -- & $*$ \\
\hline Do. & 0200 & 4 & 840 & 1,309 & 2.0 & $*$ \\
\hline Do. & 0230 & 4 & 870 & ---- & -- & $*$ \\
\hline Do. & 0300 & 4 & 900 & ---- & --- & $*$ \\
\hline Do. & 0330 & 4 & 930 & ---- & 3.3 & $*$ \\
\hline Do. & 0400 & 4 & 960 & ---- & --- & $*$ \\
\hline Do. & 0500 & 4 & 1,020 & 741 & --- & $*$ \\
\hline Do. & 0600 & 4 & 1,080 & ---- & --- & $*$ \\
\hline Do. & 0700 & 4 & 1,140 & ---- & --- & $*$ \\
\hline Do. & 0800 & 4 & 1,200 & ---- & 1.5 & $*$ \\
\hline Do. & 0900 & 4 & 1,260 & ----- & --- & $*$ \\
\hline Do. & 1000 & 4 & 1,320 & 566 & 2.0 & $*$ \\
\hline Do. & 1200 & 5 & 0 & ---- & --- & $*$ \\
\hline Do. & 1400 & 5 & 120 & 861 & --- & $*$ \\
\hline Do. & 1800 & 5 & 360 & ----- & --- & $*$ \\
\hline Do. & 1945 & 5 & 465 & ---- & --- & $*$ \\
\hline Do. & 2010 & 5 & 490 & 402 & 2.1 & $\dot{x}$ \\
\hline Do. & 2300 & 5 & 660 & ---- & --- & $*$ \\
\hline
\end{tabular}


Table 50.--Data obtained from the tracer experiments conducted during August 8-15, 1978, at the Stanton, Tex. site--Continued

\begin{tabular}{|c|c|c|c|c|c|c|}
\hline \multicolumn{7}{|c|}{ 2-Meter orange point sampler } \\
\hline Date & Clock time & $\begin{array}{l}\text { Elapsed } \\
\text { (d) }\end{array}$ & $\begin{array}{l}\text { time } \\
\text { (min) }\end{array}$ & $\begin{array}{l}\text { Boron } \\
(\mu g / L)\end{array}$ & $\begin{array}{c}\text { Bromide } \\
(\mathrm{mg} / \mathrm{L})\end{array}$ & $\begin{array}{l}\mathrm{CBrClF}_{2} \\
(\mathrm{mg} / \mathrm{L})\end{array}$ \\
\hline $8-14-78$ & 1200 & Fourth tracer & experiment & started & & \\
\hline $8-14-78$ & 1200 & 6 & 0 & 380 & 0.4 & $*$ \\
\hline Do & 1215 & 6 & 15 & --- & --- & $*$ \\
\hline Do. & 1230 & 6 & 30 & 310 & 4.5 & $*$ \\
\hline Do. & 1245 & 6 & 45 & -- & --- & $*$ \\
\hline Do. & 1300 & 6 & 60 & 330 & 11.0 & $*$ \\
\hline Do. & 1315 & 6 & 75 & -- & --- & $*$ \\
\hline Do. & 1330 & 6 & 90 & 270 & 11.0 & $*$ \\
\hline Do. & 1345 & 6 & 105 & -- & --- & $*$ \\
\hline Do. & 1400 & 6 & 120 & 290 & 8.5 & $*$ \\
\hline Do. & 1430 & 6 & 150 & 260 & ---- & $*$ \\
\hline Do. & 1500 & 6 & 180 & 260 & 5.7 & $*$ \\
\hline Do. & 1530 & 6 & 210 & --- & ---- & $*$ \\
\hline Do. & 1600 & 6 & 240 & 240 & 3.3 & $*$ \\
\hline Do. & 1630 & 6 & 270 & --- & --- & $*$ \\
\hline Do. & 1700 & 6 & 300 & -- & ---- & $*$ \\
\hline Do. & 1730 & 6 & 330 & 260 & ---- & $*$ \\
\hline Do. & 1800 & 6 & 360 & -- & --- & $*$ \\
\hline Do. & 1900 & 6 & 420 & -- & --- & $*$ \\
\hline Do. & 2000 & 6 & 480 & -- & --- & $*$ \\
\hline Do. & 2100 & 6 & 540 & -- & ---- & $*$ \\
\hline Do. & 2200 & 6 & 600 & -- & --- & $*$ \\
\hline Do. & 2300 & 6 & 660 & -- & --- & $*$ \\
\hline Do. & 2400 & 6 & 720 & 220 & 1.5 & $*$ \\
\hline
\end{tabular}


Table 50.--Data obtained from the tracer experiments conducted during August 8-15, 1978, at the Stanton, Tex. site--Continued

\begin{tabular}{|c|c|c|c|c|c|c|}
\hline \multicolumn{7}{|c|}{ 2-Meter orange point sampler } \\
\hline Date & Clock time & $\begin{array}{l}\text { Elapsed } \\
\text { (d) }\end{array}$ & $\begin{array}{l}\text { time } \\
\text { (min) }\end{array}$ & $\begin{array}{l}\text { Boron } \\
(\mu g / L)\end{array}$ & $\begin{array}{c}\text { Bromide } \\
(\mathrm{mg} / \mathrm{L})\end{array}$ & $\begin{array}{l}\mathrm{CBrClF}_{2} \\
(\mathrm{mg} / \mathrm{L})\end{array}$ \\
\hline $8-14-78$ & 1200 & Fourth tracer & experiment & = starte & ontinued & \\
\hline $8-15-78$ & 0200 & 6 & 840 & --- & --- & $*$ \\
\hline Do. & 0400 & 6 & 960 & -- & --- & $*$ \\
\hline Do. & 0600 & 6 & 1,080 & --- & --- & 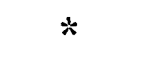 \\
\hline Do. & 0800 & 6 & 1,200 & --- & --- & $*$ \\
\hline Do. & 1000 & 6 & 1,320 & 220 & 1.5 & $*$ \\
\hline Do. & 1200 & 7 & 0 & -- & --- & $*$ \\
\hline Do. & 1400 & 7 & 120 & --- & --- & $*$ \\
\hline Do. & 1800 & 7 & 360 & 160 & 1.5 & $*$ \\
\hline
\end{tabular}


Table 51.--Data obtained from the tracer experiments conducted during August 8-15, 1978, at the Stanton, Tex. site

[Samples are from the observation well located $5 \mathrm{~m}$ from the injection well. The 5-m well is screened for the full-saturated thickness of the aquifer. The dash $(-)$ indicates sample is available but was not analyzed; an asterisk (*) indicates the sample was not analyzed and is no longer available.]

\begin{tabular}{|c|c|c|c|c|c|c|}
\hline \multicolumn{7}{|c|}{ 5-Meter wel1 } \\
\hline Date & Clock time & $\begin{array}{l}\text { Elapsed } \\
\text { (d) }\end{array}$ & $\begin{array}{l}\text { time } \\
\text { (min) }\end{array}$ & $\begin{array}{l}\text { Boron } \\
(\mu \mathrm{g} / \mathrm{L})\end{array}$ & $\begin{array}{c}\text { Bromide } \\
(\mathrm{mg} / \mathrm{L})\end{array}$ & $\begin{array}{l}\mathrm{CBrClF}_{2} \\
(\mathrm{mg} / \mathrm{L})\end{array}$ \\
\hline $8-8-78$ & 1200 & First tracer & experiment & started & & \\
\hline $8-8-78$ & 1200 & 0 & 0 & 105 & --- & $*$ \\
\hline Do. & 1210 & 0 & 10 & --- & --- & $*$ \\
\hline Do. & 1220 & 0 & 20 & ---- & 0.3 & 0.01 \\
\hline Do. & 1230 & 0 & 30 & 101 & --- & $*$ \\
\hline Do. & 1240 & 0 & 40 & --- & .4 & $*$ \\
\hline Do. & 1250 & 0 & 50 & ---- & --- & .02 \\
\hline Do. & 1300 & 0 & 60 & 386 & 3.0 & * \\
\hline Do. & 1310 & 0 & 70 & ----- & ---- & $*$ \\
\hline Do. & 1320 & 0 & 80 & ---- & ---- & $*$ \\
\hline Do. & 1330 & 0 & 90 & 650 & 4.8 & .34 \\
\hline Do. & 1340 & 0 & 100 & --- & ---- & $*$ \\
\hline Do. & 1350 & 0 & 110 & ---- & ---- & $*$ \\
\hline Do. & 1400 & 0 & 120 & 964 & 8.0 & .40 \\
\hline Do. & 1410 & 0 & 130 & ---- & --- & $*$ \\
\hline Do. & 1420 & 0 & 140 & ---- & --- & $*$ \\
\hline Do. & 1430 & 0 & 150 & 1,271 & 11.3 & .98 \\
\hline Do. & 1440 & 0 & 160 & -- - & ---- & $*$ \\
\hline Do. & 1450 & 0 & 170 & ---- & ---- & $*$ \\
\hline Do . & 1500 & 0 & 180 & 1,325 & 11.9 & 1.20 \\
\hline Do. & 1510 & 0 & 190 & ---- & --- & $*$ \\
\hline Do. & 1520 & 0 & 200 & --- & --- & $*$ \\
\hline
\end{tabular}


Table 51.--Data obtained from the tracer experiments conducted during August 8-15, 1978, at the Stanton, Tex. site--Continued

\begin{tabular}{|c|c|c|c|c|c|c|c|}
\hline \multicolumn{8}{|c|}{ 5-Meter we11 } \\
\hline Date & Clock time & & $\begin{array}{l}\text { Elapsed } \\
\text { (d) }\end{array}$ & $\begin{array}{l}\text { time } \\
\text { (min) }\end{array}$ & $\begin{array}{l}\text { Boron } \\
(\mu \mathrm{g} / \mathrm{L})\end{array}$ & $\begin{array}{c}\text { Bromide } \\
(\mathrm{mg} / \mathrm{L})\end{array}$ & $\begin{array}{l}\mathrm{CBrC} \mathrm{F}_{2} \\
(\mathrm{mg} / \mathrm{L})\end{array}$ \\
\hline $8-8-78$ & 1200 & First & tracer & experiment & started & ntinued & \\
\hline $8-8-78$ & 1530 & & 0 & 210 & 1,352 & --- & 1.22 \\
\hline Do. & 1540 & & 0 & 220 & $-\cdots$ & $-\cdots$ & 1.85. \\
\hline Do. & 1550 & & 0 & 230 & $-\cdots$ & --- & $*$ \\
\hline Do. & 1600 & & 0 & 240 & 1,866 & 17.5 & $*$ \\
\hline Do. & 1630 & & 0 & 270 & 1,721 & 15.7 & 1.64 \\
\hline Do. & 1700 & & 0 & 300 & 3,160 & 28.7 & .86 \\
\hline Do. & 1730 & & 0 & 330 & 1,853 & 17.0 & $*$ \\
\hline Do. & 1800 & & 0 & 360 & 1,630 & 14.5 & $*$ \\
\hline Do. & 1815 & & 0 & 375 & $-\cdots$ & $-\cdots$ & $*$ \\
\hline Do. & 1830 & & 0 & 390 & 1,819 & 17.5 & .05 \\
\hline Do. & 1845 & & 0 & 405 & $-\cdots$ & --- & $*$ \\
\hline Do. & 1900 & & 0 & 420 & $-\cdots$ & --- & $*$ \\
\hline Do. & 1915 & & 0 & 435 & 1,962 & 19.4 & $*$ \\
\hline Do. & 1930 & & 0 & 450 & $\cdots-$ & $-\infty$ & .04 \\
\hline Do. & 1945 & & 0 & 465 & 1,716 & 15.7 & $\star$ \\
\hline Do. & 2000 & & 0 & 480 & $\cdots$ & --- & .04 \\
\hline Do. & 2020 & & 0 & 500 & $\cdots$ & $-\cdots$ & $*$ \\
\hline Do. & 2030 & & 0 & 510 & 1,320 & 12.5 & $*$ \\
\hline Do. & 2045 & & 0 & 525 & $\cdots-$ & $-\infty$ & * \\
\hline Do. & 2100 & & 0 & 540 & 1,156 & 9.4 & $*$ \\
\hline Do. & 2120 & & 0 & 560 & - - & $-\cdots$ & $*$ \\
\hline Do. & 2140 & & 0 & 580 & 973 & 6.7 & $*$ \\
\hline Do. & 2200 & & 0 & 600 & 916 & 6.4 & .04 \\
\hline Do. & 2230 & & 0 & 630 & 826 & 6.5 & $*$ \\
\hline Do. & 2300 & & 0 & 660 & 561 & 4.2 & $*$ \\
\hline Do. & 2400 & & 0 & 720 & 670 & 5.1 & .19 \\
\hline
\end{tabular}


Table 51.--Data obtained from the tracer experiments conducted during August 8-15, 1978, at the Stanton, Tex. site--Continued

\begin{tabular}{|c|c|c|c|c|c|c|}
\hline \multicolumn{7}{|c|}{ 5-Meter well } \\
\hline Date & Clock time & $\begin{array}{l}\text { Elapsed } \\
\text { (d) }\end{array}$ & $\begin{array}{l}\text { time } \\
(\min )\end{array}$ & $\begin{array}{l}\text { Boron } \\
(\mu \mathrm{g} / \mathrm{L})\end{array}$ & $\begin{array}{r}\text { Bromide } \\
(\mathrm{mg} / \mathrm{L})\end{array}$ & $\begin{array}{c}\mathrm{CBrClF}_{2} \\
(\mathrm{mg} / \mathrm{L})\end{array}$ \\
\hline $8-8-78$ & \multicolumn{6}{|c|}{ First tracer experiment started--Continued } \\
\hline $8-9-78$ & 0200 & 0 & 840 & 502 & 3.5 & 0.13 \\
\hline Do. & 0400 & 0 & 960 & 444 & 2.9 & .06 \\
\hline Do. & 0600 & 0 & 1,080 & 382 & 2.3 & $*$ \\
\hline Do. & 0815 & 0 & 1,215 & 350 & 1.9 & $*$ \\
\hline Do. & 1000 & 0 & 1,320 & -- & 1.6 & $*$ \\
\hline Do. & 1200 & 1 & 0 & 299 & 1.5 & $*$ \\
\hline Do. & 1415 & 1 & 15 & --- & 1.3 & * \\
\hline Do. & 1600 & 1 & 240 & 263 & 1.2 & $*$ \\
\hline Do. & 1800 & 1 & 360 & -- & 1.1 & .01 \\
\hline $8-10-78$ & 0605 & 1 & 1,085 & -- & -- & $*$ \\
\hline Do. & 0815 & 1 & 1,215 & 269 & --- & $*$ \\
\hline Do . & 1000 & 1 & 1,320 & -- & 1.0 & $*$ \\
\hline $8-10-78$ & 1300 & Second tracer & exper & t started & & \\
\hline $8-10-78$ & 1310 & 2 & 70 & 222 & -- & * \\
\hline Do. & 1320 & 2 & 80 & -- & --- & $*$ \\
\hline Do. & 1330 & 2 & 90 & -- & 1.1 & $*$ \\
\hline Do & 1340 & 2 & 100 & -- & -- & $*$ \\
\hline Do. & 1350 & 2 & 110 & -- & -- & $*$ \\
\hline Do. & 1401 & 2 & 121 & 224 & 1.2 & $*$ \\
\hline Do. & 1410 & 2 & 130 & --- & -- & $*$ \\
\hline Do. & 1420 & 2 & 140 & --- & 1.6 & $*$ \\
\hline Do. & 1430 & 2 & 150 & --- & 3.2 & $*$ \\
\hline Do. & 1445 & 2 & 165 & --- & --- & $*$ \\
\hline Do. & 1500 & 2 & 180 & -- & 3.3 & $*$ \\
\hline Do. & 1515 & 2 & 195 & -- & 6.2 & $*$ \\
\hline
\end{tabular}


Table 51.--Data obtained from the tracer experiments conducted during August 8-15, 1978, at the Stanton, Tex. site--Continued

\begin{tabular}{|c|c|c|c|c|c|c|}
\hline \multicolumn{7}{|c|}{ 5-Meter wel1 } \\
\hline Date & Clock time & $\begin{array}{l}\text { Elapsed } \\
\text { (d) }\end{array}$ & $\begin{array}{l}\text { time } \\
\text { (min) }\end{array}$ & $\begin{array}{l}\text { Boron } \\
(\mu g / L)\end{array}$ & $\begin{array}{r}\text { Bromide } \\
(\mathrm{mg} / \mathrm{L})\end{array}$ & $\begin{array}{c}\mathrm{CBrClF}_{2} \\
(\mathrm{mg} / \mathrm{L})\end{array}$ \\
\hline $8-10-78$ & 1300 & Second tracer & experiment & starte & ontinued & \\
\hline $8-10-78$ & 1530 & 2 & 210 & --- & 5.3 & $*$ \\
\hline Do. & 1545 & 2 & 225 & --- & --- & $*$ \\
\hline Do. & 1600 & 2 & 240 & 228 & 10.5 & $*$ \\
\hline Do. & 1630 & 2 & 270 & -- & 8.3 & $*$ \\
\hline Do. & 1700 & 2 & 300 & --- & ---- & $*$ \\
\hline Do. & 1800 & 2 & 360 & --- & 11.3 & $*$ \\
\hline Do. & 1900 & 2 & 420 & -- & 10.5 & $*$ \\
\hline Do. & 2000 & 2 & 480 & 216 & --- & $*$ \\
\hline Do. & 2010 & 2 & 490 & -- & --- & $*$ \\
\hline Do. & 2100 & 2 & 540 & --- & 6.2 & $*$ \\
\hline Do. & 2210 & 2 & 610 & -- & 5.4 & $*$ \\
\hline Do. & 2215 & 2 & 615 & --- & --- & $*$ \\
\hline Do. & 2310 & 2 & 670 & -- & 4.3 & $*$ \\
\hline Do. & 2320 & 2 & 680 & --- & --- & $*$ \\
\hline Do. & 2400 & 2 & 720 & 216 & 3.4 & $*$ \\
\hline $8-11-78$ & 0030 & 2 & 750 & --- & --- & $*$ \\
\hline Do. & 0100 & 2 & 780 & -- & $-\cdots$ & $*$ \\
\hline Do. & 0205 & 2 & 845 & --- & --- & $*$ \\
\hline Do. & 0225 & 2 & 865 & $\cdots$ & --- & $*$ \\
\hline Do. & 0305 & 2 & 905 & -- & $\cdots$ & $*$ \\
\hline Do. & 0315 & 2 & 915 & $-\infty$ & --- & $*$ \\
\hline Do. & 0405 & 2 & 965 & --- & 2.7 & $*$ \\
\hline Do. & 0415 & 2 & 975 & -- & --- & $*$ \\
\hline Do. & 0600 & 2 & 1,080 & 222 & $\cdots$ & $*$ \\
\hline Do. & 0800 & 2 & 1,200 & -- & 2.6 & $*$ \\
\hline Do. & 1010 & 2 & 1,330 & -- & --- & $*$ \\
\hline
\end{tabular}


Table 51.--Data obtained from the tracer experiments conducted during August 8-15, 1978, at the Stanton, Tex. site--Continued

\begin{tabular}{|c|c|c|c|c|c|c|c|}
\hline \multicolumn{8}{|c|}{ 5-Meter wel1 } \\
\hline Date & Clock time & & $\begin{array}{l}\text { Elapsed } \\
\text { (d) }\end{array}$ & $\begin{array}{l}\text { time } \\
\text { (min) }\end{array}$ & $\begin{array}{l}\text { Boron } \\
(\mu \mathrm{g} / \mathrm{L})\end{array}$ & $\begin{array}{r}\text { Bromide } \\
(\mathrm{mg} / \mathrm{L})\end{array}$ & $\begin{array}{l}\mathrm{CBrClF}_{2} \\
(\mathrm{mg} / \mathrm{L})\end{array}$ \\
\hline $8-10-78$ & 1300 & Second & tracer & - experiment & $=$ starte & Continued & \\
\hline $8-11-78$ & 1020 & & 2 & 1,340 & -- & -- & * \\
\hline Do. & 1200 & & 3 & 0 & 210 & 2.0 & $*$ \\
\hline Do. & 1210 & & 3 & 10 & -- & -- & $*$ \\
\hline Do. & 1400 & & 3 & 120 & --- & -- & $*$ \\
\hline Do. & 1408 & & 3 & 128 & --- & -- & $*$ \\
\hline Do. & 1555 & & 3 & 235 & -- & -- & $*$ \\
\hline Do. & 1805 & & 3 & 365 & 206 & 2.2 & $*$ \\
\hline Do. & 2100 & & 3 & 540 & -- & -- & $*$ \\
\hline Do. & 2400 & & 3 & 720 & -- & 2.0 & $*$ \\
\hline $8-12-78$ & 0600 & & 3 & 1,080 & 192 & -- & $*$ \\
\hline Do. & 1043 & & 3 & 1,363 & 220 & 2.0 & $*$ \\
\hline $8-12-78$ & 1710 & Third & tracer & experiment & started & & \\
\hline $8-12-78$ & 1710 & & 4 & 310 & 192 & -- & $*$ \\
\hline Do. & 1730 & & 4 & 330 & 190 & --- & $*$ \\
\hline Do. & 1800 & & 4 & 360 & 216 & 1.9 & $*$ \\
\hline Do. & 1815 & & 4 & 375 & -- & --- & $*$ \\
\hline Do. & 1830 & & 4 & 390 & 384 & -- & $*$ \\
\hline Do. & 1845 & & 4 & 405 & 520 & -- & $*$ \\
\hline Do. & 1900 & & 4 & 420 & -- & -- & $*$ \\
\hline Do. & 1915 & & 4 & 435 & --- & --- & $*$ \\
\hline Do. & 1930 & & 4 & 450 & -- & --- & $*$ \\
\hline Do. & 1945 & & 4 & 465 & 880 & --- & $*$ \\
\hline Do. & 2000 & & 4 & 480 & --- & --- & $*$ \\
\hline Do. & 2030 & & 4 & 510 & -- & -- & $*$ \\
\hline Do. & 2100 & & 4 & 540 & --- & 2.1 & $*$ \\
\hline
\end{tabular}


Table 51.--Data obtained from the tracer experiments conducted during August 8-15, 1978, at the Stanton, Tex. site--Continued

\begin{tabular}{|c|c|c|c|c|c|c|c|}
\hline \multicolumn{8}{|c|}{ 5-Meter we11 } \\
\hline Date & Clock time & & $\begin{array}{l}\text { Elapsed } \\
\text { (d) }\end{array}$ & $\begin{array}{l}\text { time } \\
\text { (min) }\end{array}$ & $\begin{array}{l}\text { Boron } \\
(\mu \mathrm{g} / \mathrm{L})\end{array}$ & $\begin{array}{r}\text { Bromide } \\
(\mathrm{mg} / \mathrm{L})\end{array}$ & $\begin{array}{c}\mathrm{CBrClF}_{2} \\
(\mathrm{mg} / \mathrm{L})\end{array}$ \\
\hline $8-12-78$ & 1710 & Third & tracer & experiment & started & ntinued & \\
\hline $8-12-78$ & 2130 & & 4 & 570 & 1,402 & --- & $*$ \\
\hline Do. & 2200 & & 4 & 600 & $-\cdots$ & -- & $*$ \\
\hline Do. & 2230 & & 4 & 630 & 1,526 & -- & $*$ \\
\hline Do. & 2300 & & 4 & 660 & --- & -- & $*$ \\
\hline Do. & 2330 & & 4 & 690 & $-\cdots-$ & -- & $*$ \\
\hline Do. & 2400 & & 4 & 720 & 1,699 & 2.3 & $*$ \\
\hline $8-13-78$ & 0030 & & 4 & 750 & 1,718 & -- & $*$ \\
\hline Do. & 0100 & & 4 & 780 & ---- & -- & $*$ \\
\hline Do. & 0130 & & 4 & 810 & $-\cdots$ & -- & $*$ \\
\hline Do. & 0200 & & 4 & 840 & $-\cdots$ & -- & $*$ \\
\hline Do. & 0230 & & 4 & 870 & 1,171 & -- & $*$ \\
\hline Do. & 0300 & & 4 & 900 & $-\cdots$ & 1.2 & $*$ \\
\hline Do. & 0330 & & 4 & 930 & ---- & -- & $*$ \\
\hline Do. & 0400 & & 4 & 960 & 896 & -- & $*$ \\
\hline Do. & 0500 & & 4 & 1,020 & $-\cdots$ & --- & $*$ \\
\hline Do. & 0600 & & 4 & 1,080 & 687 & 1.9 & $*$ \\
\hline Do. & 0700 & & 4 & 1,140 & $-\cdots$ & --- & $*$ \\
\hline Do. & 0800 & & 4 & 1,200 & 565 & -- & $*$ \\
\hline Do. & 0900 & & 4 & 1,260 & $-\cdots$ & $-\cdots$ & $*$ \\
\hline Do. & 1000 & & 4 & 1,320 & 496 & -- & $*$ \\
\hline Do. & 1200 & & 5 & 0 & 421 & 1.9 & $*$ \\
\hline Do. & 1400 & & 5 & 120 & 387 & --- & $*$ \\
\hline Do. & 1600 & & 5 & 240 & 353 & -- & $*$ \\
\hline Do. & 2000 & & 5 & 480 & 315 & 1.7 & $*$ \\
\hline $8-14-78$ & 0600 & & 5 & 1,080 & 276 & -- & $*$ \\
\hline
\end{tabular}


Table 51.--Data obtained from the tracer experiments conducted during August 8-15, 1978, at the Stanton, Tex. site--Continued

\begin{tabular}{|c|c|c|c|c|c|c|}
\hline \multicolumn{7}{|c|}{ 5-Meter well } \\
\hline Date & Clock time & $\begin{array}{l}\text { Elapsed } \\
\text { (d) }\end{array}$ & $\begin{array}{l}\text { time } \\
\text { (min) }\end{array}$ & $\begin{array}{l}\text { Boron } \\
(\mu \mathrm{g} / \mathrm{L})\end{array}$ & $\begin{array}{r}\text { Bromide } \\
(\mathrm{mg} / \mathrm{L})\end{array}$ & $\begin{array}{l}\mathrm{CBrClF}_{2} \\
(\mathrm{mg} / \mathrm{L})\end{array}$ \\
\hline $8-14-78$ & 1200 & Fourth tracer & experiment & started & & \\
\hline $8-14-78$ & 1200 & 6 & 0 & 260 & --- & $\star$ \\
\hline Do. & 1215 & 6 & 15 & --- & --- & $*$ \\
\hline Do. & 1230 & 6 & 30 & 220 & 0.3 & $*$ \\
\hline Do. & 1245 & 6 & 45 & --- & --- & $*$ \\
\hline Do. & 1300 & 6 & 60 & 220 & 2.1 & $\star$ \\
\hline Do. & 1315 & 6 & 75 & --- & --- & $*$ \\
\hline Do. & 1330 & 6 & 90 & 250 & 3.3 & $*$ \\
\hline Do. & 1345 & 6 & 105 & --- & --- & $*$ \\
\hline Do. & 1400 & 6 & 120 & 260 & 8.0 & $*$ \\
\hline Do. & 1430 & 6 & 150 & 280 & 3.5 & $*$ \\
\hline Do. & 1500 & 6 & 180 & 300 & 3.2 & $*$ \\
\hline Do. & 1530 & 6 & 210 & 270 & 3.3 & $\star$ \\
\hline Do. & 1600 & 6 & 240 & 260 & 3.0 & $*$ \\
\hline Do. & 1630 & 6 & 270 & --- & --- & $*$ \\
\hline Do. & 1700 & 6 & 300 & 270 & 1.8 & $*$ \\
\hline Do. & 1730 & 6 & 330 & --- & --- & $*$ \\
\hline Do. & 1800 & 6 & 360 & --- & --- & $*$ \\
\hline Do. & 1900 & 6 & 420 & --- & --- & $*$ \\
\hline Do. & 2000 & 6 & 480 & --- & --- & $*$ \\
\hline Do. & 2100 & 6 & 540 & --- & --- & $*$ \\
\hline Do. & 2200 & 6 & 600 & 250 & 1.0 & $*$ \\
\hline Do. & 2300 & 6 & 660 & --- & --- & $*$ \\
\hline Do. & 2400 & 6 & 720 & --- & --- & $*$ \\
\hline $8-15-78$ & 0200 & 6 & 840 & --- & --- & $*$ \\
\hline Do. & 0400 & 6 & 960 & 240 & 1.0 & $*$ \\
\hline
\end{tabular}


Table 51.--Data obtained from the tracer experiments conducted during August 8-15, 1978, at the Stanton, Tex. site--Continued

\begin{tabular}{|c|c|c|c|c|c|c|}
\hline \multicolumn{7}{|c|}{ 5-Meter wel1 } \\
\hline Date & Clock time & $\begin{array}{l}\text { Elapsed } \\
\text { (d) }\end{array}$ & $\begin{array}{l}\text { time } \\
(\min )\end{array}$ & $\begin{array}{l}\text { Boron } \\
(\mu \mathrm{g} / \mathrm{L})\end{array}$ & $\begin{array}{c}\text { Bromide } \\
(\mathrm{mg} / \mathrm{L})\end{array}$ & $\begin{array}{c}\mathrm{CBrClF}_{2} \\
(\mathrm{mg} / \mathrm{L})\end{array}$ \\
\hline $8-14-78$ & 1200 & Fourth tracer & exper & t starte & Continued & \\
\hline $8-15-78$ & 0600 & 6 & 1,080 & -- & -- & $*$ \\
\hline Do. & 0800 & 6 & 1,200 & -- & -- & $*$ \\
\hline Do. & 1000 & 6 & 1,320 & 230 & 1.0 & $*$ \\
\hline Do. & 1200 & 7 & 0 & -- & -- & $*$ \\
\hline Do. & 1400 & 7 & 120 & -- & -- & $*$ \\
\hline Do. & 1800 & 7 & 360 & 220 & 3.4 & $*$ \\
\hline
\end{tabular}


Table 52.--Data obtained from the tracer experiments conducted during August 8-15, 1978, at the Stanton, Tex. site

[Samples are from the point sampler designated 5-m black located $5 \mathrm{~m}$ radially from the injection well and $41.8 \mathrm{~m}$ below land surface. The dash (-) indicates sample is available but was not analyzed; an asterisk (*) indicates the sample was not analyzed and is no longer available.]

\begin{tabular}{|c|c|c|c|c|c|c|}
\hline & & 5-Meter b] & Lack point & sampler & & \\
\hline Date & Clock time & $\begin{array}{l}\text { Elapsed } \\
\text { (d) }\end{array}$ & $\begin{array}{l}\text { time } \\
\text { (min) }\end{array}$ & $\begin{array}{l}\text { Boron } \\
(\mu g / L)\end{array}$ & $\begin{array}{c}\text { Bromide } \\
(\mathrm{mg} / \mathrm{L})\end{array}$ & $\begin{array}{c}\mathrm{CBrClF}_{2} \\
(\mathrm{mg} / \mathrm{L})\end{array}$ \\
\hline $8-8-78$ & 1200 & First tracer & experiment & started & & \\
\hline $8-8-78$ & 1200 & 0 & 0 & 178 & --- & $*$ \\
\hline Do. & 1210 & 0 & 10 & -- & --- & $*$ \\
\hline Do. & 1220 & 0 & 20 & --- & 3.0 & $\neq$ \\
\hline Do. & 1230 & 0 & 30 & -- & --- & $*$ \\
\hline Do. & 1240 & 0 & 40 & -- & --- & $*$ \\
\hline Do. & 1250 & 0 & 50 & --- & --- & $*$ \\
\hline Do. & 1300 & 0 & 60 & 178 & --- & * \\
\hline Do. & 1310 & 0 & 70 & --- & 5.2 & $*$ \\
\hline Do. & 1320 & 0 & 80 & -- & --- & $*$ \\
\hline Do. & 1330 & 0 & 90 & --- & --- & $*$ \\
\hline Do. & 1340 & 0 & 100 & --- & --- & $*$ \\
\hline Do. & 1350 & 0 & 110 & --- & --- & $*$ \\
\hline Do. & 1400 & 0 & 120 & 158 & --- & $*$ \\
\hline Do. & 1410 & 0 & 130 & --- & 5.4 & 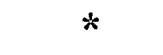 \\
\hline Do. & 1420 & 0 & 140 & --- & --- & $\star$ \\
\hline Do. & 1430 & 0 & 150 & --- & --- & $*$ \\
\hline Do. & 1440 & 0 & 160 & --- & --- & $\star$ \\
\hline Do. & 1450 & 0 & 170 & --- & --- & $*$ \\
\hline Do. & 1500 & 0 & 180 & 159 & --- & $\star$ \\
\hline Do. & 1510 & 0 & 190 & --- & --- & $*$ \\
\hline Do. & 1520 & 0 & 200 & -- & 9.1 & $*$ \\
\hline Do. & 1530 & 0 & 210 & -- & --- & $*$ \\
\hline
\end{tabular}


Table 52.--Data obtained from the tracer experiments conducted during August 8-15, 1978, at the Stanton, Tex. site--Continued

\begin{tabular}{|c|c|c|c|c|c|c|c|}
\hline \multicolumn{8}{|c|}{ 5-Meter black point sampler } \\
\hline Date & Clock time & & $\begin{array}{l}\text { Elapsed } \\
\text { (d) }\end{array}$ & $\begin{array}{l}\text { time } \\
\text { (min) }\end{array}$ & $\begin{array}{l}\text { Boron } \\
(\mu g / L)\end{array}$ & $\begin{array}{c}\text { Bromide } \\
(\mathrm{mg} / \mathrm{L})\end{array}$ & $\begin{array}{c}\mathrm{CBrClF}_{2} \\
(\mathrm{mg} / \mathrm{L})\end{array}$ \\
\hline $8-8-78$ & 1200 & First & tracer & experiments & starte & ontinued & \\
\hline $8-8-78$ & 1540 & & 0 & 220 & -- & -- & $*$ \\
\hline Do. & 1550 & & 0 & 230 & -- & -- & * \\
\hline Do. & 1600 & & 0 & 240 & 248 & -- & $*$ \\
\hline Do. & 1630 & & 0 & 290 & -- & 5.4 & * \\
\hline Do. & 1700 & & 0 & 300 & -- & -- & $\star$ \\
\hline Do. & 1730 & & 0 & 330 & --- & 5.8 & $*$ \\
\hline Do. & 1800 & & 0 & 360 & 148 & -- & $*$ \\
\hline Do. & 1815 & & 0 & 375 & -- & -- & $*$ \\
\hline Do. & 1830 & & 0 & 390 & -- & 6.7 & $*$ \\
\hline Do. & 1845 & & 0 & 405 & --- & --- & $*$ \\
\hline Do. & 1900 & & 0 & 420 & -- & -- & $\star$ \\
\hline Do. & 1915 & & 0 & 435 & -- & -- & $*$ \\
\hline Do. & 1930 & & 0 & 450 & -- & 5.1 & $*$ \\
\hline Do. & 1945 & & 0 & 465 & -- & -- & $*$ \\
\hline Do. & 2000 & & 0 & 480 & 152 & -- & $*$ \\
\hline Do. & 2020 & & 0 & 500 & --- & $-\cdots$ & $*$ \\
\hline Do. & 2030 & & 0 & 510 & --- & -- & $*$ \\
\hline Do. & 2045 & & 0 & 525 & -- & -- & * \\
\hline Do. & 2100 & & 0 & 540 & -- & 9.2 & $\star$ \\
\hline Do. & 2120 & & 0 & 560 & -- & -- & $*$ \\
\hline Do. & 2140 & & 0 & 580 & --- & --- & $*$ \\
\hline Do. & 2200 & & 0 & 600 & 138 & --- & $*$ \\
\hline Do. & 2230 & & 0 & 630 & -- & -- & $\star$ \\
\hline Do. & 2300 & & 0 & 660 & -- & 5.6 & * \\
\hline Do. & 2400 & & 0 & 720 & 144 & -- & $\star$ \\
\hline $8-9-78$ & 0200 & & 0 & 840 & 154 & -- & $*$ \\
\hline
\end{tabular}


Table 52.--Data obtained from the tracer experiments conducted during August 8-15, 1978, at the Stanton, Tex. site--Continued

\begin{tabular}{|c|c|c|c|c|c|c|}
\hline & & 5-Meter $\mathrm{b}$ & olack point & sampler & & \\
\hline Date & Clock time & $\begin{array}{l}\text { Elapsed } \\
\text { (d) }\end{array}$ & $\begin{array}{l}\text { time } \\
\text { (min) }\end{array}$ & $\begin{array}{l}\text { Boron } \\
(\mu \mathrm{g} / \mathrm{L})\end{array}$ & $\begin{array}{c}\text { Bromide } \\
(\mathrm{mg} / \mathrm{L})\end{array}$ & $\begin{array}{c}\mathrm{CBrClF}_{2} \\
(\mathrm{mg} / \mathrm{L})\end{array}$ \\
\hline $8-8-78$ & 1200 & First tracer & experiment & started-- & ntinued & \\
\hline $8-9-78$ & 0400 & 0 & 960 & --- & 7.0 & $*$ \\
\hline Do. & 0600 & 0 & 1,080 & 140 & --- & $*$ \\
\hline Do. & 0815 & 0 & 1,215 & 194 & ---- & $*$ \\
\hline Do. & 1000 & 0 & 1,320 & --- & 9.9 & $*$ \\
\hline Do. & 1200 & 1 & 0 & 210 & 11.5 & $*$ \\
\hline Do. & 1400 & 1 & 120 & --- & 11.9 & $*$ \\
\hline Do. & 1415 & 1 & 135 & --- & --- & $*$ \\
\hline Do. & 1600 & 1 & 240 & 196 & 13.1 & $*$ \\
\hline Do. & 1800 & 1 & 360 & --- & 16.2 & $*$ \\
\hline Do. & 2130 & 1 & 570 & 276 & 15.5 & $*$ \\
\hline Do. & 2400 & 1 & 720 & -- & 14.3 & $*$ \\
\hline $8-10-78$ & 0600 & 1 & 1,080 & 350 & 13.1 & $*$ \\
\hline Do. & 0605 & 1 & 1,085 & -- & -- & $*$ \\
\hline Do. & 0800 & 1 & 1,200 & -- & $-\cdots$ & $*$ \\
\hline $8-10-78$ & 1310 & Second tracer & experiment & : started & & \\
\hline $8-10-78$ & 1310 & 2 & 70 & 286 & 12.6 & $*$ \\
\hline Do. & 1400 & 2 & 120 & 200 & 7.7 & $*$ \\
\hline Do. & 1600 & 2 & 240 & 184 & 8.9 & $*$ \\
\hline Do. & 2000 & 2 & 480 & 208 & 3.7 & $*$ \\
\hline Do. & 2400 & 2 & 720 & 206 & 5.4 & $*$ \\
\hline $8-11-78$ & 0600 & 2 & 1,080 & 155 & 6.9 & $*$ \\
\hline Do. & 1200 & 3 & 0 & 206 & 6.1 & $*$ \\
\hline Do. & 1800 & 3 & 360 & 214 & 6.1 & $*$ \\
\hline Do. & 2100 & 3 & 540 & 223 & 6.1 & $*$ \\
\hline $8-12-78$ & 0600 & 3 & 1,080 & 212 & 5.8 & $*$ \\
\hline
\end{tabular}


Table 52.--Data obtained from the tracer experiment conducted during August 8-15, 1978, at the Stanton, Tex. site--Continued

\begin{tabular}{|c|c|c|c|c|c|c|}
\hline & & 5-Meter $b$ & lack point & sampler & & \\
\hline Date & Clock time & $\begin{array}{l}\text { Elapsed } \\
\text { (d) }\end{array}$ & $\begin{array}{l}\text { time } \\
\text { (min) }\end{array}$ & $\begin{array}{l}\text { Boron } \\
(\mu g / L)\end{array}$ & $\begin{array}{c}\text { Bromide } \\
(\mathrm{mg} / \mathrm{L})\end{array}$ & $\begin{array}{l}\mathrm{CBrClF}_{2} \\
(\mathrm{mg} / \mathrm{L})\end{array}$ \\
\hline $8-12-78$ & 1710 & Third tracer & experiment & started & & \\
\hline $8-12-78$ & 1730 & 4 & 330 & 212 & 4.4 & $*$ \\
\hline Do. & 1900 & 4 & 420 & 178 & 5.2 & $*$ \\
\hline Do. & 2200 & 4 & 600 & 167 & 7.2 & $*$ \\
\hline Do. & 2400 & 4 & 720 & 159 & 5.2 & $*$ \\
\hline $8-13-78$ & 0600 & 4 & 1,080 & --- & --- & $*$ \\
\hline Do. & 1200 & 5 & 0 & 176 & 4.1 & $*$ \\
\hline Do. & 1800 & 5 & 360 & 208 & 6.6 & $*$ \\
\hline Do. & 2300 & 5 & 660 & 191 & 8.9 & * \\
\hline $8-14-78$ & 0300 & 5 & 900 & 202 & 9.6 & $*$ \\
\hline Do. & 0600 & 5 & 1,080 & 230 & 6.1 & $*$ \\
\hline $8-14-78$ & 1200 & Fourth tracer & experiment & started & & \\
\hline $8-14-78$ & 1200 & 6 & 0 & 180 & .4 & * \\
\hline Do. & 1215 & 6 & 15 & -- & -- & $*$ \\
\hline Do. & 1230 & 6 & 30 & 270 & 1.5 & $*$ \\
\hline Do. & 1245 & 6 & 45 & --- & -- & * \\
\hline Do. & 1300 & 6 & 60 & 220 & 1.2 & $*$ \\
\hline Do. & 1315 & 6 & 75 & --- & --- & * \\
\hline Do. & 1330 & 6 & 90 & 230 & 1.7 & $*$ \\
\hline Do. & 1345 & 6 & 105 & -- & -- & $*$ \\
\hline Do. & 1400 & 6 & 120 & 210 & .9 & $*$ \\
\hline Do. & 1430 & 6 & 150 & -- & --- & $*$ \\
\hline Do. & 1500 & 6 & 180 & 220 & .7 & $*$ \\
\hline Do. & 1530 & 6 & 210 & --- & -- & $*$ \\
\hline Do. & 1600 & 6 & 240 & 220 & 1.0 & $*$ \\
\hline Do. & 1630 & 6 & 270 & -- & -- & $*$ \\
\hline
\end{tabular}


Table 52.--Data obtained from the tracer experiments conducted during August 8-15, 1978, at the Stanton, Tex. site--Continued

\begin{tabular}{|c|c|c|c|c|c|c|}
\hline \multicolumn{7}{|c|}{ 5-Meter black point sampler } \\
\hline Date & Clock time & $\begin{array}{l}\text { Elapsed } \\
\text { (d) }\end{array}$ & $\begin{array}{l}\text { time } \\
\text { (min) }\end{array}$ & $\begin{array}{l}\text { Boron } \\
(\mu \mathrm{g} / \mathrm{L})\end{array}$ & $\begin{array}{r}\text { Bromide } \\
(\mathrm{mg} / \mathrm{L})\end{array}$ & $\begin{array}{c}\mathrm{CBrClF}_{2} \\
(\mathrm{mg} / \mathrm{L})\end{array}$ \\
\hline $8-14-78$ & 1200 & Fourth tracer & experiment & starte & ontinued & \\
\hline $8-14-78$ & 1700 & 6 & 300 & -- & -- & $*$ \\
\hline Do & 1730 & 6 & 330 & -- & -- & $*$ \\
\hline Do & 1800 & 6 & 360 & 200 & 1.0 & $*$ \\
\hline Do. & 1900 & 6 & 420 & -- & -- & $*$ \\
\hline Do $\cdot$ & 2000 & 6 & 480 & -- & -- & $*$ \\
\hline Do. & 2100 & 6 & 540 & --- & --- & $*$ \\
\hline Do. & 2200 & 6 & 600 & --- & --- & $*$ \\
\hline Do. & 2300 & 6 & 660 & -- & -- & $*$ \\
\hline Do. & 2400 & 6 & 720 & 220 & 1.2 & $*$ \\
\hline $8-15-78$ & 0200 & 6 & 840 & -- & -- & $*$ \\
\hline Do. & 0400 & 6 & 960 & 240 & 1.5 & $*$ \\
\hline Do. & 0600 & 6 & 1,080 & --- & -- & $*$ \\
\hline Do. & 0800 & 6 & 1,200 & --- & -- & $*$ \\
\hline Do. & 1000 & 6 & 1,320 & 290 & 1.2 & $*$ \\
\hline Do. & 1200 & 7 & 0 & --- & --- & $*$ \\
\hline Do. & 1400 & 7 & 120 & -- & -- & $*$ \\
\hline Do. & 1800 & 7 & 360 & 240 & .7 & $\star$ \\
\hline
\end{tabular}


Table 53.--Data obtained from the tracer experiments conducted during August 8-15, 1978, at the Stanton, Tex. site

[Samples are from an observation well designated 5-m red and screened only for $0.7 \mathrm{~m}$ at $40.0 \mathrm{~m}$ below land surface. This sampler is located at a distance of $5 \mathrm{~m}$ radially from the injection well. The dash (-) indicates sample is available but was not analyzed; an asterisk (*) indicates the sample was not analyzed and is no longer available.]

\begin{tabular}{|c|c|c|c|c|c|c|}
\hline \multicolumn{7}{|c|}{ 5-Meter red point well } \\
\hline Date & Clock time & $\begin{array}{l}\text { Elapsed } \\
\text { (d) }\end{array}$ & $\begin{array}{l}\text { time } \\
\text { (min) }\end{array}$ & $\begin{array}{l}\text { Boron } \\
(\mu \mathrm{g} / \mathrm{L})\end{array}$ & $\begin{array}{c}\text { Bromide } \\
(\mathrm{mg} / \mathrm{L})\end{array}$ & $\begin{array}{l}\mathrm{CBrClF}_{2} \\
(\mathrm{mg} / \mathrm{L})\end{array}$ \\
\hline $8-8-78$ & 1200 & First tracer & experiment & started & & \\
\hline $8-8-78$ & 1200 & 0 & 0 & ---- & $-\cdots$ & $*$ \\
\hline Do. & 1210 & 0 & 10 & $-\cdots-$ & $-\cdots$ & $*$ \\
\hline Do. & 1220 & 0 & 20 & 95 & 0.6 & $*$ \\
\hline Do. & 1230 & 0 & 30 & $\cdots--$ & 3.6 & 0.01 \\
\hline Do. & 1240 & 0 & 40 & 1,044 & 10.9 & $*$ \\
\hline Do $\cdot$ & 1250 & 0 & 50 & $-\cdots$ & 13.5 & .11 \\
\hline Do. & 1300 & 0 & 60 & 1,609 & 17.0 & $*$ \\
\hline Do. & 1310 & 0 & 70 & $\cdots--$ & 18.3 & $*$ \\
\hline Do. & 1320 & 0 & 80 & 1,889 & 19.6 & .41 \\
\hline Do. & 1330 & 0 & 90 & $-\cdots$ & 20.7 & $*$ \\
\hline Do. & 1340 & 0 & 100 & 2,021 & 22.1 & $*$ \\
\hline Do. & 1350 & 0 & 110 & $\ldots$ & 23.0 & $*$ \\
\hline Do. & 1400 & 0 & 120 & 2,234 & 23.3 & $*$ \\
\hline Do. & 1410 & 0 & 130 & $\ldots$ & 24.0 & $*$ \\
\hline Do. & 1420 & 0 & 140 & 2,383 & 24.7 & $*$ \\
\hline Do. & 1430 & 0 & 150 & $\cdots$ & 25.0 & 2.6 \\
\hline Do. & 1440 & 0 & 160 & 2,549 & 26.5 & $*$ \\
\hline Do. & 1450 & 0 & 170 & $\cdots$ & 28.5 & $*$ \\
\hline Do. & 1500 & 0 & 180 & 2,697 & 29.0 & 1.5 \\
\hline
\end{tabular}


Table 53.--Data obtained from the tracer experiments conducted during August 8-15, 1978, at the Stanton, Tex. site--Continued

\begin{tabular}{|c|c|c|c|c|c|c|}
\hline \multicolumn{7}{|c|}{ 5-Meter red point well } \\
\hline Date & Clock time & $\begin{array}{l}\text { Elapsed } \\
\text { (d) }\end{array}$ & $\begin{array}{l}\text { time } \\
\text { (min) }\end{array}$ & $\begin{array}{l}\text { Boron } \\
(\mu \mathrm{g} / \mathrm{L})\end{array}$ & $\begin{array}{c}\text { Bromide } \\
(\mathrm{mg} / \mathrm{L})\end{array}$ & $\begin{array}{c}\mathrm{CBrC} \mathrm{F}_{2} \\
(\mathrm{mg} / \mathrm{L})\end{array}$ \\
\hline $8-8-78$ & 1200 & First tracer & experiment & starte & ontinued & \\
\hline $8-8-78$ & 1510 & 0 & 190 & ---- & 29.0 & * \\
\hline Do. & 1520 & 0 & 200 & 2,760 & 30.0 & $*$ \\
\hline Do. & 1530 & 0 & 210 & --- & 31.0 & 0.92 \\
\hline Do. & 1540 & 0 & 220 & 2,739 & --- & 1.0 \\
\hline Do. & 1550 & 0 & 230 & --- & 31.5 & $*$ \\
\hline Do. & 1600 & 0 & 240 & 2,866 & $\cdots-$ & $*$ \\
\hline Do. & 1630 & 0 & 270 & 2,908 & 33.0 & .59 \\
\hline Do. & 1700 & 0 & 300 & ---- & ---- & 1.8 \\
\hline Do. & 1730 & 0 & 330 & 3,121 & 34.0 & $\star$ \\
\hline Do. & 1800 & 0 & 360 & 3,181 & 35.0 & $\star$ \\
\hline Do. & 1815 & 0 & 375 & --- & 34.5 & $\star$ \\
\hline Do. & 1830 & 0 & 390 & 2,846 & 29.6 & $\star$ \\
\hline Do. & 1845 & 0 & 405 & --- & --- & $*$ \\
\hline Do. & 1900 & 0 & 420 & ---- & 16.9 & $*$ \\
\hline Do. & 1915 & 0 & 435 & 1,460 & 15.1 & $*$ \\
\hline Do. & 1930 & 0 & 450 & 1,340 & 14.0 & $*$ \\
\hline Do. & 1945 & 0 & 465 & ---- & --- & $*$ \\
\hline Do. & 2000 & 0 & 480 & 1,100 & 11.5 & $*$ \\
\hline Do. & 2020 & 0 & 500 & $-\cdots$ & 10.3 & $*$ \\
\hline Do. & 2030 & 0 & 510 & 980 & 9.6 & $\star$ \\
\hline Do. & 2045 & 0 & 525 & $-\cdots$ & 9.6 & $*$ \\
\hline Do. & 2100 & 0 & 540 & 840 & --- & $*$ \\
\hline Do. & 2120 & 0 & 560 & --- & 7.8 & $*$ \\
\hline Do. & 2140 & 0 & 580 & 700 & 7.1 & $\star$ \\
\hline Do. & 2200 & 0 & 600 & $-\cdots$ & $-\cdots$ & .38 \\
\hline Do. & 2230 & 0 & 630 & 520 & 5.5 & $*$ \\
\hline
\end{tabular}


Table 53.--Data obtained from the tracer experiments conducted during August 8-15, 1978, at the Stanton, Tex. site--Continued

\begin{tabular}{|c|c|c|c|c|c|c|}
\hline \multicolumn{7}{|c|}{ 5-Meter red point well } \\
\hline Date & Clock time & $\begin{array}{l}\text { Elapsed } \\
\text { (d) }\end{array}$ & $\begin{array}{l}\text { time } \\
\text { (min) }\end{array}$ & $\begin{array}{l}\text { Boron } \\
(\mu g / L)\end{array}$ & $\begin{array}{r}\text { Bromide } \\
(\mathrm{mg} / \mathrm{L})\end{array}$ & $\begin{array}{l}\mathrm{CBrClF}_{2} \\
(\mathrm{mg} / \mathrm{L})\end{array}$ \\
\hline $8-8-78$ & 1200 & First tracer & experiment & started-- & ntinued & \\
\hline $8-8-78$ & 2300 & 0 & 660 & -- & 6.8 & $*$ \\
\hline Do. & 2400 & 0 & 720 & 472 & 3.7 & 0.37 \\
\hline $8-9-78$ & 0200 & 0 & 840 & 324 & 2.4 & .37 \\
\hline Do. & 0400 & 0 & 960 & 258 & 1.8 & .02 \\
\hline Do. & 0600 & 0 & 1,080 & 206 & 1.4 & $*$ \\
\hline Do. & 0815 & 0 & 1,215 & 170 & 1.0 & $*$ \\
\hline Do. & 1000 & 0 & 1,320 & --- & 1.1 & $*$ \\
\hline Do. & 1200 & 1 & 0 & 162 & ---- & $*$ \\
\hline Do. & 1415 & 1 & 215 & --- & .7 & $*$ \\
\hline Do. & 1600 & 1 & 320 & -- & ---- & $*$ \\
\hline Do. & 1800 & 1 & 440 & -- & .6 & .01 \\
\hline Do. & 2130 & 1 & 650 & 125 & --- & .01 \\
\hline $8-10-78$ & 0615 & 1 & 1,175 & --- & .4 & $*$ \\
\hline Do. & 0810 & 1 & 1,295 & -- & .4 & $*$ \\
\hline $8-10-78$ & 1300 & Second tracer & experiment & started & & \\
\hline $8-10-78$ & 1300 & 2 & 60 & 110 & ---- & $*$ \\
\hline Do. & 1320 & 2 & 80 & --- & .7 & $*$ \\
\hline Do. & 1330 & 2 & 90 & --- & 2.2 & $*$ \\
\hline Do. & 1340 & 2 & 100 & --- & 8.1 & $*$ \\
\hline Do. & 1350 & 2 & 110 & --- & 12.5 & $*$ \\
\hline Do. & 1400 & 2 & 120 & 110 & ---- & $*$ \\
\hline Do. & 1410 & 2 & 130 & --- & 10.9 & $*$ \\
\hline Do. & 1420 & 2 & 140 & --- & 12.6 & $*$ \\
\hline Do. & 1430 & 2 & 150 & --- & 13.2 & $*$ \\
\hline Do. & 1445 & 2 & 165 & --- & ---- & $*$ \\
\hline
\end{tabular}


Table 53.--Data obtained from the tracer experiments conducted during August 8-15, 1978, at the Stanton, Tex. site--Continued

\begin{tabular}{|c|c|c|c|c|c|c|}
\hline \multicolumn{7}{|c|}{ 5-Meter red point well } \\
\hline Date & Clock time & $\begin{array}{l}\text { Elapsed } \\
\text { (d) }\end{array}$ & $\begin{array}{l}\text { time } \\
\text { (min) }\end{array}$ & $\begin{array}{l}\text { Boron } \\
(\mu \mathrm{g} / \mathrm{L})\end{array}$ & $\begin{array}{c}\text { Bromide } \\
(\mathrm{mg} / \mathrm{L})\end{array}$ & $\begin{array}{l}\mathrm{CBrClF}_{2} \\
(\mathrm{mg} / \mathrm{L})\end{array}$ \\
\hline $8-10-78$ & 1300 & Second tracer & experiment & starte & ontinued & \\
\hline $8-10-78$ & 1500 & 2 & 180 & --- & --- & $*$ \\
\hline Do. & 1515 & 2 & 195 & --- & 12.8 & $*$ \\
\hline Do. & 1530 & 2 & 210 & -- & 12.7 & $*$ \\
\hline Do. & 1545 & 2 & 225 & --- & 12.7 & * \\
\hline Do. & 1600 & 2 & 240 & 108 & 14.8 & $*$ \\
\hline Do. & 1630 & 2 & 270 & --- & ---- & $*$ \\
\hline Do. & 1700 & 2 & 300 & --- & --- & $*$ \\
\hline Do. & 1730 & 2 & 330 & --- & --- & $*$ \\
\hline Do. & 1805 & 2 & 365 & --- & 4.4 & $*$ \\
\hline Do. & 1900 & 2 & 420 & --- & 3.2 & $*$ \\
\hline Do. & 2000 & 2 & 480 & 102 & ---- & $*$ \\
\hline Do. & 2115 & 2 & 555 & --- & 1.7 & $*$ \\
\hline Do. & 2210 & 2 & 610 & --- & 1.4 & $*$ \\
\hline Do. & 2310 & 2 & 670 & 108 & ---- & $*$ \\
\hline $8-11-78$ & 0005 & 2 & 725 & --- & 1.3 & $*$ \\
\hline Do. & 0100 & 2 & 780 & -- & ---- & $*$ \\
\hline Do. & 0215 & 2 & 855 & --- & ---- & $*$ \\
\hline Do. & 0310 & 2 & 910 & --- & ---- & $*$ \\
\hline Do. & 0410 & 2 & 970 & --- & --- & $*$ \\
\hline Do. & 0600 & 2 & 1,080 & 102 & .8 & $*$ \\
\hline Do. & 0800 & 2 & 1,200 & -- & --- & $*$ \\
\hline Do. & 1025 & 2 & 1,345 & --- & ---- & $*$ \\
\hline Do. & 1206 & 3 & 6 & 102 & .5 & $*$ \\
\hline Do. & 1406 & 3 & 126 & --- & --- & $*$ \\
\hline Do. & 1610 & 3 & 250 & --- & ---- & $*$ \\
\hline Do. & 1735 & 3 & 335 & -- & --- & $*$ \\
\hline Do. & 1810 & 3 & 370 & 102 & --- & $*$ \\
\hline
\end{tabular}


Table 53.--Data obtained from the tracer experiments conducted during August 8-15, 1978, at the Stanton, Tex. site--Continued

5-Meter red point well

\begin{tabular}{|c|c|c|c|c|c|c|}
\hline Date & Clock time & $\begin{array}{l}\text { Elapsed } \\
\text { (d) }\end{array}$ & $\begin{array}{l}\text { time } \\
\text { (min) }\end{array}$ & $\begin{array}{l}\text { Boron } \\
(\mu \mathrm{g} / \mathrm{L})\end{array}$ & $\begin{array}{c}\text { Bromide } \\
(\mathrm{mg} / \mathrm{L})\end{array}$ & $\begin{array}{c}\mathrm{CBrC1F}_{2} \\
(\mathrm{mg} / \mathrm{L})\end{array}$ \\
\hline $8-10-78$ & 1300 & Second tracer & experiments & s start & -Continued & \\
\hline $8-11-78$ & 2100 & 3 & 540 & ---- & --- & $\star$ \\
\hline Do. & 2130 & 3 & 570 & ---- & -- & $*$ \\
\hline Do. & 2400 & 3 & 720 & 98 & 0.4 & $*$ \\
\hline $8-12-78$ & 1048 & 3 & 1,368 & 109 & .5 & $*$ \\
\hline Do. & 1700 & 4 & 300 & ---- & .5 & $*$ \\
\hline
\end{tabular}

\begin{tabular}{|c|c|c|c|c|c|c|c|}
\hline $8-12-78$ & 1710 & Third & tracer & experiments & s started & & \\
\hline $8-12-78$ & 1735 & & 4 & 335 & $>1,544$ & --- & $*$ \\
\hline Do. & 1802 & & 4 & 362 & $>2,128$ & -- & * \\
\hline Do. & 1817 & & 4 & 377 & ---- & --- & $*$ \\
\hline Do. & 1845 & & 4 & 405 & ---- & --- & $*$ \\
\hline Do. & 1904 & & 4 & 424 & $>2,600$ & --- & * \\
\hline Do. & 1915 & & 4 & 435 & ---- & -- & $*$ \\
\hline Do. & 1930 & & 4 & 450 & $>2,702$ & -- & $*$ \\
\hline Do. & 1945 & & 4 & 465 & ---- & .6 & $*$ \\
\hline Do. & 2000 & & 4 & 480 & 2,968 & --- & $*$ \\
\hline Do. & 2030 & & 4 & 510 & 3,086 & -- & $*$ \\
\hline Do. & 2100 & & 4 & 540 & 3,142 & -- & $*$ \\
\hline Do. & 2200 & & 4 & 600 & 3,130 & --- & $*$ \\
\hline Do. & 2230 & & 4 & 630 & ---- & -- & * \\
\hline Do. & 2300 & & 4 & 660 & 3,352 & -- & $*$ \\
\hline Do. & 2330 & & 4 & 690 & --- & .7 & * \\
\hline Do. & 2400 & & 4 & 720 & 2,568 & --- & $*$ \\
\hline $8-13-78$ & 0030 & & 4 & 750 & ---- & --- & $*$ \\
\hline Do. & 0100 & & 4 & 780 & 1,144 & -- & $*$ \\
\hline Do. & 0130 & & 4 & 810 & ---- & -- & * \\
\hline Do. & 0200 & & 4 & 840 & 894 & -- & $*$ \\
\hline
\end{tabular}


Table 53.--Data obtained from the tracer experiments conducted during August 8-15, 1978, at the Stanton, Tex. site--Continued

\begin{tabular}{|c|c|c|c|c|c|c|}
\hline & & 5-Meter & red point & we11 & & \\
\hline Date & Clock time & $\begin{array}{l}\text { Elapsed } \\
\text { (d) }\end{array}$ & $\begin{array}{l}\text { time } \\
\text { (min) }\end{array}$ & $\begin{array}{l}\text { Boron } \\
(\mu g / L)\end{array}$ & $\begin{array}{c}\text { Bromide } \\
(\mathrm{mg} / \mathrm{L})\end{array}$ & $\begin{array}{l}\mathrm{CBrClF}_{2} \\
(\mathrm{mg} / \mathrm{L})\end{array}$ \\
\hline $8-12-78$ & 1710 & Third tracer & experiment & started-- & ntinued & \\
\hline $8-13-78$ & 0230 & 4 & 870 & -- & --- & $*$ \\
\hline Do. & 0300 & 4 & 900 & 681 & --- & $*$ \\
\hline Do. & 0330 & 4 & 930 & -- & --- & $*$ \\
\hline Do. & 0400 & 4 & 960 & 558 & --- & $*$ \\
\hline Do. & 0500 & 4 & 1,020 & --- & 0.7 & $*$ \\
\hline Do. & 0600 & 4 & 1,020 & 385 & --- & $*$ \\
\hline Do. & 0700 & 4 & 1,140 & -- & --- & $\star$ \\
\hline Do. & 0800 & 4 & 1,200 & 291 & ---- & $*$ \\
\hline Do. & 0900 & 4 & 1,260 & -- & ---- & $*$ \\
\hline Do. & 1000 & 4 & 1,340 & -- & --- & $*$ \\
\hline Do. & 1200 & 4 & 0 & 218 & 0.5 & $*$ \\
\hline Do. & 1400 & 4 & 120 & -- & --- & $*$ \\
\hline Do. & 1810 & 4 & 370 & -- & --- & $*$ \\
\hline Do. & 2012 & 4 & 492 & 161 & --- & $*$ \\
\hline Do. & 2300 & 4 & 660 & 149 & .4 & $*$ \\
\hline $8-14-78$ & 0610 & 4 & 1,090 & -- & .4 & $*$ \\
\hline $8-14-78$ & 1200 & Fourth tracer & experiment & started & & \\
\hline $8-14-78$ & 1200 & 5 & 0 & 260 & 1.7 & $*$ \\
\hline Do. & 1215 & 6 & 15 & 140 & 7.5 & $*$ \\
\hline Do. & 1230 & 6 & 30 & 140 & 70.1 & $*$ \\
\hline Do. & 1245 & 6 & 45 & 130 & 27.4 & $*$ \\
\hline Do. & 1300 & 6 & 60 & 120 & 10.0 & $*$ \\
\hline Do. & 1315 & 6 & 75 & 130 & 11.0 & $*$ \\
\hline Do. & 1330 & 6 & 90 & 110 & 10.0 & $*$ \\
\hline Do. & 1345 & 6 & 105 & 120 & 7.0 & $*$ \\
\hline Do. & 1400 & 6 & 120 & 120 & 5.5 & $*$ \\
\hline
\end{tabular}


Table 53.--Data obtained from the tracer experiments conducted during August 8-15, 1978, at the Stanton, Tex. site--Continued

\begin{tabular}{|c|c|c|c|c|c|c|}
\hline \multicolumn{7}{|c|}{ 5-Meter red point well } \\
\hline Date & Clock time & $\begin{array}{l}\text { Elapsed } \\
\text { (d) }\end{array}$ & $\begin{array}{l}\text { time } \\
\text { (min) }\end{array}$ & $\begin{array}{l}\text { Boron } \\
(\mu \mathrm{g} / \mathrm{L})\end{array}$ & $\begin{array}{r}\text { Bromide } \\
(\mathrm{mg} / \mathrm{L})\end{array}$ & $\begin{array}{l}\mathrm{CBrClF} \\
(\mathrm{mg} / \mathrm{L})\end{array}$ \\
\hline $8-14-78$ & 1200 & Fourth tracer & experiment & $t$ starte & Continued & \\
\hline $8-14-78$ & 1430 & 6 & 150 & 110 & 4.6 & $*$ \\
\hline Do. & 1500 & 6 & 180 & 110 & 4.0 & $*$ \\
\hline Do. & 1530 & 6 & 210 & -- & ---- & $*$ \\
\hline Do. & 1600 & 6 & 240 & -- & --- & $*$ \\
\hline Do . & 1630 & 6 & 270 & --- & --- & $*$ \\
\hline Do. & 1700 & 6 & 300 & -- & --- & $*$ \\
\hline Do. & 1730 & 6 & 330 & --- & ---- & $*$ \\
\hline Do & 1800 & 6 & 360 & 110 & 1.5 & $*$ \\
\hline Do & 1900 & 6 & 420 & -- & ---- & $*$ \\
\hline Do & 2000 & 6 & 480 & -- & ---- & $*$ \\
\hline Do. & 2100 & 6 & 540 & -- & ---- & $*$ \\
\hline Do. & 2200 & 6 & 600 & 110 & 0.6 & $*$ \\
\hline Do. & 2300 & 6 & 660 & --- & --- & $*$ \\
\hline Do. & 2400 & 6 & 720 & --- & ---- & $*$ \\
\hline $8-15-78$ & 0200 & 7 & 840 & --- & ---- & $*$ \\
\hline Do. & 0400 & 7 & 960 & 100 & 2.5 & $*$ \\
\hline Do. & 0600 & 7 & 1,080 & -- & ---- & $*$ \\
\hline Do. & 0800 & 7 & 1,200 & -- & ---- & $*$ \\
\hline Do. & 1000 & 7 & 1,320 & 130 & --- & $*$ \\
\hline Do. & 1200 & 7 & 0 & --- & ---- & $*$ \\
\hline Do. & 1400 & 7 & 120 & -- & ---- & $*$ \\
\hline Do. & 1800 & 7 & 480 & 120 & .9 & $*$ \\
\hline
\end{tabular}


Table 54.--Data obtained from the tracer experiments conducted during August 8-15, 1978, at the Stanton, Tex. site

[Samples are from the point sampler designated 5-m orange located $37.5 \mathrm{~m}$ below land surface and $5 \mathrm{~m}$ radially from the injection well. The dash (-) indicates sample is available but was not analyzed; an asterisk (*) indicates the sample was not analyzed and is no longer available.]

\begin{tabular}{|c|c|c|c|c|c|c|c|}
\hline \multicolumn{8}{|c|}{ 5-Meter orange point sampler } \\
\hline Date & Clock time & & $\begin{array}{l}\text { Elapsed } \\
\text { (d) }\end{array}$ & $\begin{array}{l}\text { time } \\
\text { (min) }\end{array}$ & $\begin{array}{l}\text { Boron } \\
(\mu g / L)\end{array}$ & $\begin{array}{c}\text { Bromide } \\
(\mathrm{mg} / \mathrm{L})\end{array}$ & $\begin{array}{c}\mathrm{CBrClF}_{2} \\
(\mathrm{mg} / \mathrm{L})\end{array}$ \\
\hline $8-8-78$ & 1200 & First & tracer & experiment & started & & \\
\hline $8-8-78$ & 1200 & & 0 & 0 & 130 & 0.3 & $*$ \\
\hline Do. & 1210 & & 0 & 10 & --- & --- & $*$ \\
\hline Do. & 1220 & & 0 & 20 & --- & --- & $*$ \\
\hline Do . & 1230 & & 0 & 30 & -- & --- & $*$ \\
\hline Do. & 1240 & & 0 & 40 & -- & --- & $*$ \\
\hline Do. & 1250 & & 0 & 50 & --- & --- & $*$ \\
\hline Do. & 1300 & & 0 & 60 & 99 & .5 & $*$ \\
\hline Do. & 1310 & & 0 & 70 & -- & --- & $*$ \\
\hline Do. & 1320 & & 0 & 80 & --- & ---- & $*$ \\
\hline Do. & 1330 & & 0 & 90 & 107 & 1.1 & 0.01 \\
\hline Do. & 1400 & & 0 & 120 & 119 & 3.5 & .03 \\
\hline Do. & 1410 & & 0 & 130 & -- & --- & $*$ \\
\hline Do. & 1420 & & 0 & 140 & -- & --- & $*$ \\
\hline Do. & 1430 & & 0 & 150 & 142 & 7.2 & .08 \\
\hline Do. & 1440 & & 0 & 160 & --- & --- & $*$ \\
\hline Do. & 1450 & & 0 & 170 & -- & ---- & $*$ \\
\hline Do. & 1500 & & 0 & 180 & 166 & 12.1 & .22 \\
\hline Do. & 1510 & & 0 & 190 & --- & ---- & $*$ \\
\hline Do. & 1520 & & 0 & 200 & --- & ---- & $\star$ \\
\hline Do. & 1530 & & 0 & 210 & --- & ---- & $*$ \\
\hline Do. & 1540 & & 0 & 220 & 324 & 12.8 & .27 \\
\hline
\end{tabular}


Table 54.--Data obtained from the tracer experiments conducted during August 8-15, 1978, at the Stanton, Tex. site--Continued

\begin{tabular}{|c|c|c|c|c|c|c|c|}
\hline \multicolumn{8}{|c|}{ 5-Meter orange point sampler } \\
\hline Date & Clock time & & $\begin{array}{l}\text { Elapsed } \\
\text { (d) }\end{array}$ & $\begin{array}{l}\text { time } \\
\text { (min) }\end{array}$ & $\begin{array}{l}\text { Boron } \\
(\mu \mathrm{g} / \mathrm{L})\end{array}$ & $\begin{array}{c}\text { Bromide } \\
(\mathrm{mg} / \mathrm{L})\end{array}$ & $\begin{array}{c}\mathrm{CBrClF}_{2} \\
(\mathrm{mg} / \mathrm{L})\end{array}$ \\
\hline $8-8-78$ & 1200 & First & tracer & experiment & started & ntinued & \\
\hline $8-8-78$ & 1550 & & 0 & 230 & ---- & --- & $*$ \\
\hline Do. & 1600 & & 0 & 240 & ---- & --- & $*$ \\
\hline Do. & 1630 & & 0 & 270 & 305 & 17.0 & 0.65 \\
\hline Do. & 1700 & & 0 & 300 & 372 & 18.3 & .86 \\
\hline Do. & 1730 & & 0 & 330 & 431 & 19.5 & .55 \\
\hline Do. & 1800 & & 0 & 360 & 521 & 21.2 & .56 \\
\hline Do. & 1815 & & 0 & 375 & ----- & --- & $*$ \\
\hline Do. & 1830 & & 0 & 390 & 672 & 25.7 & .28 \\
\hline Do. & 1845 & & 0 & 405 & ---- & ---- & $*$ \\
\hline Do. & 1900 & & 0 & 420 & 922 & 28.3 & .72 \\
\hline Do. & 1915 & & 0 & 435 & ----- & --- & $*$ \\
\hline Do. & 1930 & & 0 & 450 & 1,044 & 27.4 & .58 \\
\hline Do. & 1945 & & 0 & 465 & ---- & $-\cdots$ & $*$ \\
\hline Do. & 2000 & & 0 & 480 & 1,188 & 24.5 & .70 \\
\hline Do. & 2020 & & 0 & 500 & ---- & ---- & $*$ \\
\hline Do. & 2030 & & 0 & 510 & ---- & ---- & .21 \\
\hline Do. & 2045 & & 0 & 525 & ---- & --- & $*$ \\
\hline Do. & 2100 & & 0 & 540 & 1,416 & 18.0 & .17 \\
\hline Do. & 2120 & & 0 & 560 & ---- & --- & $*$ \\
\hline Do. & 2140 & & 0 & 580 & ---- & --- & $*$ \\
\hline Do. & 2200 & & 0 & 600 & 1,483 & 13.9 & .10 \\
\hline Do. & 2230 & & 0 & 630 & ---- & ---- & $*$ \\
\hline Do. & 2300 & & 0 & 660 & 1,366 & 12.4 & $*$ \\
\hline Do. & 2400 & & 0 & 720 & 1,314 & 9.9 & $*$ \\
\hline $8-9-78$ & 0200 & & 0 & 840 & 1,220 & 7.6 & .08 \\
\hline Do. & 0400 & & 0 & 960 & 1,146 & 4.9 & .09 \\
\hline
\end{tabular}


Table 54.--Data obtained from the tracer experiments conducted during August 8-15, 1978, at the Stanton, Tex. site--Continued

\begin{tabular}{|c|c|c|c|c|c|c|}
\hline \multicolumn{7}{|c|}{ 5-Meter orange point sampler } \\
\hline Date & Clock time & $\begin{array}{l}\text { Elapsed } \\
\text { (d) }\end{array}$ & $\begin{array}{l}\text { time } \\
\text { (min) }\end{array}$ & $\begin{array}{l}\text { Boron } \\
(\mu \mathrm{g} / \mathrm{L})\end{array}$ & $\begin{array}{c}\text { Bromide } \\
(\mathrm{mg} / \mathrm{L})\end{array}$ & $\begin{array}{c}\mathrm{CBrClF}_{2} \\
(\mathrm{mg} / \mathrm{L})\end{array}$ \\
\hline $8-8-78$ & 1200 & First tracer & experiment & started- & ntinued & \\
\hline $8-9-78$ & 0600 & 0 & 1,080 & 1,027 & 3.3 & $*$ \\
\hline Do. & 0815 & 0 & 1,215 & 891 & 2.7 & 0.04 \\
\hline Do. & 1000 & 0 & 1,320 & --- & 2.7 & .02 \\
\hline Do. & 1200 & 1 & 0 & 750 & 1.8 & $*$ \\
\hline Do. & 1415 & 1 & 135 & --- & 1.7 & $*$ \\
\hline Do. & 1600 & 1 & 240 & --- & 1.8 & $*$ \\
\hline Do. & 1800 & 1 & 360 & $-\cdots-$ & 1.7 & $*$ \\
\hline Do. & 2130 & 1 & 570 & 443 & 1.7 & .04 \\
\hline $8-10-78$ & 0605 & 1 & 1,085 & 370 & -- & $*$ \\
\hline Do. & 0800 & 1 & 1,200 & --- & -- & $*$ \\
\hline Do . & 1255 & 2 & 55 & 251 & --- & $*$ \\
\hline $8-10-78$ & 1300 & Second tracer & experiment & : started & & \\
\hline $8-10-78$ & 1300 & 2 & 60 & $\cdots$ & .8 & $*$ \\
\hline Do. & 1310 & 2 & 70 & ---- & -- & $*$ \\
\hline Do. & 1320 & 2 & 80 & ---- & -- & $*$ \\
\hline Do. & 1330 & 2 & 90 & ---- & 1.0 & $*$ \\
\hline Do. & 1350 & 2 & 110 & 190 & -- & $*$ \\
\hline Do. & 1400 & 2 & 120 & ----- & .9 & $*$ \\
\hline Do. & 1410 & 2 & 130 & --- & --- & $*$ \\
\hline Do. & 1420 & 2 & 140 & ---- & -- & $*$ \\
\hline Do. & 1430 & 2 & 150 & ---- & 1.0 & $*$ \\
\hline Do. & 1445 & 2 & 165 & 188 & -- & $*$ \\
\hline Do. & 1500 & 2 & 180 & --- & 2.8 & $*$ \\
\hline Do. & 1515 & 2 & 195 & --- & -- & $*$ \\
\hline Do. & 1530 & 2 & 210 & ---- & 8.9 & $*$ \\
\hline
\end{tabular}


Table 54.--Data obtained from the tracer experiments conducted during August 8-15, 1978, at the Stanton, Tex. site--Continued

\begin{tabular}{|c|c|c|c|c|c|c|}
\hline \multicolumn{7}{|c|}{ 5-Meter orange point sampler } \\
\hline Date & Clock time & $\begin{array}{l}\text { Elapsed } \\
\text { (d) }\end{array}$ & $\begin{array}{l}\text { time } \\
(\min )\end{array}$ & $\begin{array}{l}\text { Boron } \\
(\mu \mathrm{g} / \mathrm{L})\end{array}$ & $\begin{array}{c}\text { Bromide } \\
(\mathrm{mg} / \mathrm{L})\end{array}$ & $\begin{array}{l}\mathrm{CBrClF}_{2} \\
(\mathrm{mg} / \mathrm{L})\end{array}$ \\
\hline $8-10-78$ & 1300 & Second tracer & experiment & \multicolumn{3}{|c|}{ started--Continued } \\
\hline $8-10-78$ & 1545 & 2 & 225 & 179 & ---- & $*$ \\
\hline Do. & 1600 & 2 & 240 & -- & 8.6 & $*$ \\
\hline Do. & 1630 & 2 & 270 & --- & 9.6 & $*$ \\
\hline Do. & 1700 & 2 & 300 & 162 & 11.5 & $*$ \\
\hline Do. & 1730 & 2 & 330 & --- & 13.2 & $*$ \\
\hline Do. & 1800 & 2 & 360 & 158 & 12.5 & $*$ \\
\hline Do. & 1900 & 2 & 420 & --- & 20.5 & $*$ \\
\hline Do. & 2000 & 2 & 480 & 154 & 15.7 & $*$ \\
\hline Do. & 2100 & 2 & 540 & --- & 13.7 & $*$ \\
\hline Do. & 2300 & 2 & 660 & 152 & 8.0 & $*$ \\
\hline $8-11-78$ & 0100 & 2 & 780 & 150 & 4.6 & $*$ \\
\hline Do. & 0204 & 2 & 844 & -- & 3.1 & $*$ \\
\hline Do. & 0300 & 2 & 900 & 145 & 2.8 & $*$ \\
\hline Do. & 0400 & 2 & 960 & --- & 2.1 & $*$ \\
\hline Do. & 0600 & 2 & 1,080 & 140 & 1.5 & $*$ \\
\hline Do. & 0800 & 2 & 1,200 & 140 & ---- & $*$ \\
\hline Do. & 1005 & 2 & 1,325 & 140 & 1.6 & $*$ \\
\hline Do. & 1200 & 3 & 0 & 138 & 1.0 & $*$ \\
\hline Do. & 1400 & 3 & 120 & 131 & ----- & $*$ \\
\hline Do. & 1600 & 3 & 240 & 133 & ---- & $*$ \\
\hline Do. & 1800 & 3 & 360 & 131 & 1.15 & $*$ \\
\hline $8-12-78$ & 0030 & 3 & 750 & -- & .7 & $*$ \\
\hline Do. & 0600 & 3 & 1,080 & 125 & .7 & $*$ \\
\hline Do. & 1035 & 3 & 1,355 & 117 & ----- & $*$ \\
\hline Do. & 1650 & 4 & 290 & --- & .6 & $*$ \\
\hline
\end{tabular}


Table 54.--Data obtained from the tracer experiments conducted during August 8-15, 1978, at the Stanton, Tex. site--Continued

\begin{tabular}{|c|c|c|c|c|c|c|}
\hline & & 5-Meter orc & ange point & sampler & & \\
\hline Date & Clock time & $\begin{array}{l}\text { Elapsed } \\
\text { (d) }\end{array}$ & $\begin{array}{l}\text { time } \\
\text { (min) }\end{array}$ & $\begin{array}{l}\text { Boron } \\
(\mu \mathrm{g} / \mathrm{L})\end{array}$ & $\begin{array}{c}\text { Bromide } \\
(\mathrm{mg} / \mathrm{L})\end{array}$ & $\begin{array}{l}\mathrm{CBrClF}_{2} \\
(\mathrm{mg} / \mathrm{L})\end{array}$ \\
\hline $8-12-78$ & 1710 & Third tracer & experiments & started & & \\
\hline $8-12-78$ & 1710 & 4 & 310 & ---- & --- & $*$ \\
\hline Do. & 1730 & 4 & 330 & 108 & -- & $*$ \\
\hline Do. & 1800 & 4 & 360 & ---- & --- & $*$ \\
\hline Do. & 1815 & 4 & 375 & --- & 0.6 & $*$ \\
\hline Do. & 1830 & 4 & 390 & 121 & -- & $*$ \\
\hline Do. & 1845 & 4 & 405 & ----- & --- & $*$ \\
\hline Do. & 1900 & 4 & 420 & ---- & --- & $*$ \\
\hline Do. & 1915 & 4 & 435 & ---- & --- & $*$ \\
\hline Do. & 1930 & 4 & 450 & 171 & --- & $*$ \\
\hline Do. & 1945 & 4 & 465 & --- & --- & $*$ \\
\hline Do. & 2000 & 4 & 480 & 234 & .5 & * \\
\hline Do. & 2100 & 4 & 540 & ---- & --- & $*$ \\
\hline Do. & 2130 & 4 & 570 & 313 & -- & $*$ \\
\hline Do. & 2200 & 4 & 600 & ---- & -- & $*$ \\
\hline Do. & 2230 & 4 & 630 & ---- & --- & * \\
\hline Do. & 2300 & 4 & 660 & $-\cdots$ & -- & $*$ \\
\hline Do. & 2330 & 4 & 690 & ---- & --- & $*$ \\
\hline Do. & 2400 & 4 & 720 & 555 & .8 & $*$ \\
\hline $8-13-78$ & 0100 & 4 & 780 & 786 & --- & $*$ \\
\hline Do. & 0130 & 4 & 810 & --- & --- & $*$ \\
\hline Do. & 0200 & 4 & 840 & $-\cdots$ & -- & $*$ \\
\hline Do. & 0230 & 4 & 870 & 1,054 & --- & $*$ \\
\hline Do. & 0300 & 4 & 900 & ---- & --- & $*$ \\
\hline Do. & 0330 & 4 & 930 & $-\cdots$ & -- & * \\
\hline Do. & 0400 & 4 & 960 & ---- & -- & $*$ \\
\hline
\end{tabular}


Table 54.--Data obtained from the tracer experiments conducted during August 8-15, 1978, at the Stanton, Tex. site--Continued

\begin{tabular}{|c|c|c|c|c|c|c|}
\hline \multicolumn{7}{|c|}{ 5-Meter orange point sampler } \\
\hline Date & Clock time & $\begin{array}{l}\text { Elapsed } \\
\text { (d) }\end{array}$ & $\begin{array}{l}\text { time } \\
\text { (min) }\end{array}$ & $\begin{array}{l}\text { Boron } \\
(\mu g / L)\end{array}$ & $\begin{array}{c}\text { Bromide } \\
(\mathrm{mg} / \mathrm{L})\end{array}$ & $\begin{array}{c}\mathrm{CBrClF}_{2} \\
(\mathrm{mg} / \mathrm{L})\end{array}$ \\
\hline $8-12-78$ & 1710 & Third tracer $\epsilon$ & experiment & started-- & ntinued & \\
\hline $8-13-78$ & 0500 & 4 & 1,020 & 1,235 & --- & $\star$ \\
\hline Do & 0600 & 4 & 1,080 & ---- & 0.8 & $*$ \\
\hline Do. & 0700 & 4 & 1,140 & 1,380 & -- & $*$ \\
\hline Do. & 0800 & 4 & 1,200 & ---- & --- & * \\
\hline Do. & 0900 & 4 & 1,260 & 1,302 & --- & * \\
\hline Do. & 1000 & 4 & 1,320 & ---- & -- & $*$ \\
\hline Do. & 1200 & 5 & 0 & 1,145 & .7 & $*$ \\
\hline Do. & 1400 & 5 & 120 & 1,207 & -- & $\star$ \\
\hline Do. & 1600 & 5 & 240 & 1,145 & -- & $*$ \\
\hline Do. & 1800 & 5 & 360 & 841 & .7 & $*$ \\
\hline Do. & 2000 & 5 & 480 & 740 & --- & $*$ \\
\hline Do . & 2300 & 5 & 540 & 628 & .6 & $*$ \\
\hline $8-14-78$ & 0300 & 5 & 900 & 643 & --- & $*$ \\
\hline Do. & 0600 & 5 & 1,080 & 468 & -- & $*$ \\
\hline $8-14-78$ & 1200 & Fourth tracer & experiment & started & & \\
\hline $8-14-78$ & 1200 & 6 & 0 & 520 & .1 & $*$ \\
\hline Do. & 1215 & 6 & 15 & ----- & -- & $*$ \\
\hline Do. & 1230 & 6 & 30 & 320 & .2 & $*$ \\
\hline Do. & 1245 & 6 & 45 & ---- & -- & $*$ \\
\hline Do. & 1300 & 6 & 60 & 330 & .2 & $*$ \\
\hline Do. & 1315 & 6 & 75 & --- & -- & $*$ \\
\hline Do & 1330 & 6 & 90 & 330 & 2.5 & $*$ \\
\hline Do. & 1345 & 6 & 105 & ---- & -- & $*$ \\
\hline Do. & 1400 & 6 & 120 & 300 & 5.5 & $*$ \\
\hline Do. & 1430 & 6 & 150 & 300 & 6.5 & 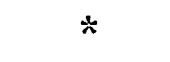 \\
\hline
\end{tabular}


Table 54.--Data obtained from the tracer experiments conducted during August 8-15, 1978, at the Stanton, Tex. site--Continued

\begin{tabular}{|c|c|c|c|c|c|c|}
\hline \multicolumn{7}{|c|}{ 5-Meter orange point sampler } \\
\hline Date & Clock time & $\begin{array}{l}\text { Elapsed } \\
\text { (d) }\end{array}$ & $\begin{array}{l}\text { time } \\
(\min )\end{array}$ & $\begin{array}{l}\text { Boron } \\
(\mu g / L)\end{array}$ & $\begin{array}{c}\text { Bromide } \\
(\mathrm{mg} / \mathrm{L})\end{array}$ & $\begin{array}{l}\mathrm{CBrClF}_{2} \\
(\mathrm{mg} / \mathrm{L})\end{array}$ \\
\hline $8-14-78$ & 1200 & Fourth tracer & experiment & starte & Continued & \\
\hline $8-14-78$ & 1500 & 6 & 180 & 280 & 7.0 & $*$ \\
\hline Do. & 1530 & 6 & 210 & 290 & 7.0 & * \\
\hline Do. & 1600 & 6 & 240 & 310 & 8.6 & $*$ \\
\hline Do. & 1630 & 6 & 270 & -- & --- & $*$ \\
\hline Do. & 1700 & 6 & 300 & -- & -- & * \\
\hline Do. & 1730 & 6 & 330 & -- & -- & $*$ \\
\hline Do. & 1800 & 6 & 360 & 260 & 7.0 & $*$ \\
\hline Do. & 1900 & 6 & 420 & -- & --- & * \\
\hline Do. & 2000 & 6 & 480 & -- & -- & $*$ \\
\hline Do. & 2100 & 6 & 540 & -- & -- & $*$ \\
\hline Do. & 2200 & 6 & 600 & -- & -- & $*$ \\
\hline Do. & 2300 & 6 & 660 & 210 & 3.8 & $*$ \\
\hline Do. & 2400 & 6 & 720 & -- & --- & $*$ \\
\hline $8-15-78$ & 0200 & 6 & 840 & --- & -- & $*$ \\
\hline Do. & 0400 & 6 & 960 & $-\cdots$ & -- & $*$ \\
\hline Do. & 0600 & 6 & 1,080 & 200 & 1.1 & $*$ \\
\hline Do. & 0800 & 6 & 1,200 & --- & -- & * \\
\hline Do. & 1000 & 6 & 1,320 & -- & -- & $*$ \\
\hline Do. & 1200 & 7 & 0 & 190 & .6 & $*$ \\
\hline Do. & 1400 & 7 & 120 & -- & -- & $*$ \\
\hline Do. & 1800 & 7 & 480 & 160 & .4 & $*$ \\
\hline
\end{tabular}


Table 55.--Data obtained from the tracer experiments conducted during August 8-15, 1978, at the Stanton, Tex. site

[Samples are from the observation well $10 \mathrm{~m}$ from the injection well and are screened only for a $1.2-\mathrm{m}$ section, $41.5 \mathrm{~m}$ below land surface. The dash (-) indicates sample is available but was not analyzed; an asterisk (*) indicates the sample was not analyzed and is no longer available.]

\begin{tabular}{|c|c|c|c|c|c|c|c|}
\hline \multicolumn{8}{|c|}{ 10-Meter well } \\
\hline Date & Clock time & & $\begin{array}{l}\text { Elapsed } \\
\text { (d) }\end{array}$ & $\begin{array}{l}\text { time } \\
\text { (min) }\end{array}$ & $\begin{array}{l}\text { Boron } \\
(\mu \mathrm{g} / \mathrm{L})\end{array}$ & $\begin{array}{c}\text { Bromide } \\
(\mathrm{mg} / \mathrm{L})\end{array}$ & $\begin{array}{c}\mathrm{CBrC1F}_{2} \\
(\mathrm{mg} / \mathrm{L})\end{array}$ \\
\hline $8-8-78$ & 1200 & First & tracer & experiment & started & & \\
\hline $8-8-78$ & 1505 & & 0 & 185 & 94 & 0.3 & $*$ \\
\hline Do. & 1600 & & 0 & 240 & 96 & .3 & $*$ \\
\hline Do. & 1904 & & 0 & 424 & 105 & .3 & $*$ \\
\hline Do. & 2002 & & 0 & 482 & -- & .8 & $*$ \\
\hline Do. & 2106 & & 0 & 546 & 196 & 2.0 & $*$ \\
\hline Do. & 2200 & & 0 & 600 & 247 & 2.8 & $*$ \\
\hline Do. & 2300 & & 0 & 660 & -- & 3.8 & $*$ \\
\hline Do. & 2400 & & 0 & 720 & 397 & 5.0 & $*$ \\
\hline $8-9-78$ & 0200 & & 0 & 840 & -- & 9.5 & $*$ \\
\hline Do. & 0400 & & 0 & 960 & 629 & 10.1 & $*$ \\
\hline Do. & 0822 & & 0 & 1,202 & -- & 9.4 & $*$ \\
\hline Do. & 1000 & & 0 & 1,320 & 621 & 8.5 & $*$ \\
\hline Do. & 1200 & & 1 & 0 & -- & 7.4 & $*$ \\
\hline Do. & 1415 & & 1 & 135 & 553 & 6.6 & $*$ \\
\hline Do. & 1602 & & 1 & 242 & --- & --- & $*$ \\
\hline Do. & 1800 & & 1 & 480 & 498 & 5.8 & $*$ \\
\hline Do. & 2130 & & 1 & 570 & --- & 4.9 & $*$ \\
\hline Do. & 2400 & & 1 & 720 & 430 & 4.6 & $*$ \\
\hline $8-10-78$ & 0610 & & 1 & 1,090 & 386 & 3.8 & $*$ \\
\hline Do. & 0815 & & 1 & 1,215 & 344 & --- & $*$ \\
\hline
\end{tabular}


Table 55.--Data obtained from the tracer experiments conducted during August 8-15, 1978, at the Stanton, Tex. site--Continued

\begin{tabular}{|c|c|c|c|c|c|c|c|}
\hline \multicolumn{8}{|c|}{ 10-Meter we11 } \\
\hline Date & Clock time & & $\begin{array}{l}\text { Elapsed } \\
\text { (d) }\end{array}$ & $\begin{array}{l}\text { time } \\
\text { (min) }\end{array}$ & $\begin{array}{l}\text { Boron } \\
(\mu \mathrm{g} / \mathrm{L})\end{array}$ & $\begin{array}{c}\text { Bromide } \\
(\mathrm{mg} / \mathrm{L})\end{array}$ & $\begin{array}{l}\mathrm{CBrClF}_{2} \\
(\mathrm{mg} / \mathrm{L})\end{array}$ \\
\hline $8-10-78$ & 1300 & Second & tracer & experiment & started & & \\
\hline $8-10-78$ & 1305 & & 2 & 65 & 312 & 2.9 & $*$ \\
\hline $8-11-78$ & 0600 & & 2 & 1,080 & 149 & 1.2 & $*$ \\
\hline Do. & 0800 & & 2 & 1,200 & 149 & 1.2 & $*$ \\
\hline Do. & 2400 & & 3 & 720 & 154 & 1.4 & $*$ \\
\hline $8-12-78$ & 0600 & & 3 & 1,080 & 154 & 1.5 & $*$ \\
\hline $8-12-78$ & 1710 & Third & tracer & experiment & started & & \\
\hline $8-12-78$ & 1735 & & 4 & 335 & -- & 1.6 & $*$ \\
\hline Do. & 1910 & & 4 & 430 & 162 & --- & $*$ \\
\hline Do. & 2100 & & 4 & 540 & 162 & 1.6 & 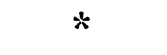 \\
\hline Do. & 2300 & & 4 & 660 & 160 & 1.2 & $*$ \\
\hline $8-13-78$ & 0100 & & 4 & 780 & 168 & 1.5 & $*$ \\
\hline Do. & 0300 & & 4 & 900 & 174 & 1.5 & $*$ \\
\hline Do. & 0500 & & 4 & 1,020 & 215 & ---- & $*$ \\
\hline Do. & 0600 & & 4 & 1,060 & --- & 1.5 & $*$ \\
\hline Do. & 0708 & & 4 & 1,148 & 314 & 1.7 & $*$ \\
\hline Do. & 0800 & & 4 & 1,208 & --- & 1.8 & $*$ \\
\hline Do. & 0908 & & 4 & 1,268 & 398 & ---- & $*$ \\
\hline Do. & 1007 & & 4 & 1,327 & --- & 1.6 & $*$ \\
\hline Do. & 1100 & & 4 & 1,380 & 475 & 1.9 & $*$ \\
\hline Do. & 1200 & & 5 & 0 & --- & 2.35 & $*$ \\
\hline Do. & 1300 & & 5 & 60 & 549 & --- & $*$ \\
\hline Do. & 1408 & & 5 & 128 & --- & ---- & $*$ \\
\hline Do. & 1500 & & 5 & 180 & 605 & 2.5 & $*$ \\
\hline Do. & 1600 & & 5 & 240 & --- & --- & $*$ \\
\hline Do. & 1800 & & 5 & 360 & 600 & 2.7 & $*$ \\
\hline
\end{tabular}


Table 55.--Data obtained from the tracer experiments conducted during August 8-15, 1978, at the Stanton, Tex. site--Continued

\begin{tabular}{|c|c|c|c|c|c|c|}
\hline \multicolumn{7}{|c|}{ 10-Meter wel1 } \\
\hline Date & Clock time & $\begin{array}{l}\text { Elapsed } \\
\text { (d) }\end{array}$ & $\begin{array}{l}\text { time } \\
(\min )\end{array}$ & $\begin{array}{l}\text { Boron } \\
(\mu \mathrm{g} / \mathrm{L})\end{array}$ & $\begin{array}{c}\text { Bromide } \\
(\mathrm{mg} / \mathrm{L})\end{array}$ & $\begin{array}{c}\mathrm{CBrClF}_{2} \\
(\mathrm{mg} / \mathrm{L})\end{array}$ \\
\hline $8-12-78$ & 1700 & Third tracer & experiment & started-- & ntinued & \\
\hline $8-13-78$ & 2012 & 5 & 612 & 598 & 2.7 & * \\
\hline Do . & 2300 & 5 & 660 & 579 & 2.9 & $*$ \\
\hline $8-14-78$ & 0300 & 5 & 900 & 544 & 2.6 & $*$ \\
\hline Do. & 0612 & 5 & 1,092 & 496 & 2.3 & $*$ \\
\hline Do. & 1000 & 5 & 1,320 & 333 & 2.0 & $*$ \\
\hline $8-14-78$ & 1200 & Fourth tracer & experiment & started & & \\
\hline $8-14-78$ & 1240 & 6 & 40 & 470 & --- & $\star$ \\
\hline Do. & 1300 & 6 & 60 & 422 & 2.6 & $*$ \\
\hline Do. & 1400 & 6 & 120 & 367 & -- & $*$ \\
\hline Do. & 1600 & 6 & 240 & 365 & -- & $*$ \\
\hline Do. & 1800 & 6 & 360 & 370 & -- & $*$ \\
\hline Do. & 2010 & 6 & 610 & 341 & -- & $*$ \\
\hline Do & 2400 & 6 & 720 & 330 & 3.7 & $*$ \\
\hline $8-15-78$ & 0200 & 6 & 840 & 309 & -- & $*$ \\
\hline Do. & 0400 & 6 & 960 & 300 & 4.2 & $*$ \\
\hline Do. & 0945 & 6 & 1,065 & 290 & 4.5 & $*$ \\
\hline Do. & 1007 & 6 & 1,327 & 278 & -- & $*$ \\
\hline Do. & 1225 & 7 & 25 & 270 & 2.6 & $*$ \\
\hline Do. & 1400 & 7 & 120 & -- & -- & $*$ \\
\hline Do. & 1800 & 7 & 360 & 270 & 1.5 & $*$ \\
\hline
\end{tabular}


Table 56.--Data obtained from the tracer experiments conducted during August 8-15, 1978, at the Stanton, Tex. site

[Samples are from the observation well $15 \mathrm{~m}$ from the injection well, which is screened for the saturated thickness of the aquifer. The dash (-) indicates sample is available but was not analyzed; an asterisk (*) indicates the sample was not analyzed and is no longer available.]

\begin{tabular}{|c|c|c|c|c|c|c|c|}
\hline \multicolumn{8}{|c|}{ 15-Meter well } \\
\hline Date & Clock tim & & $\begin{array}{l}\text { Elapsed } \\
\text { (d) }\end{array}$ & $\begin{array}{l}\text { time } \\
\text { (min) }\end{array}$ & $\begin{array}{l}\text { Boron } \\
(\mu \mathrm{g} / \mathrm{L})\end{array}$ & $\begin{array}{c}\text { Bromide } \\
(\mathrm{mg} / \mathrm{L})\end{array}$ & $\begin{array}{c}\mathrm{CBrClF}_{2} \\
(\mathrm{mg} / \mathrm{L})\end{array}$ \\
\hline $8-8-78$ & 1200 & First & tracer & experiment & started & & \\
\hline $8-8-78$ & 1204 & & 0 & 4 & 64 & 0.48 & $*$ \\
\hline Do. & 1300 & & 0 & 60 & --- & .43 & $*$ \\
\hline Do. & 1400 & & 0 & 120 & 91 & .39 & $*$ \\
\hline Do. & 1500 & & 0 & 180 & --- & .37 & $*$ \\
\hline Do. & 1600 & & 0 & 240 & 76 & .35 & $*$ \\
\hline Do. & 1800 & & 0 & 360 & 74 & .38 & $*$ \\
\hline Do. & 1815 & & 0 & 375 & --- & ---- & $*$ \\
\hline Do. & 1830 & & 0 & 390 & --- & ---- & $*$ \\
\hline Do. & 1900 & & 0 & 420 & 70 & .40 & $*$ \\
\hline Do. & 2006 & & 0 & 486 & 64 & .41 & $*$ \\
\hline Do. & 2106 & & 0 & 546 & --- & .51 & $*$ \\
\hline Do. & 2200 & & 0 & 600 & --- & .50 & $*$ \\
\hline Do. & 2300 & & 0 & 600 & 78 & .58 & $*$ \\
\hline Do. & 2400 & & 0 & 720 & -- & .62 & $*$ \\
\hline $8-9-78$ & 0200 & & 0 & 840 & --- & 1.10 & $*$ \\
\hline Do. & 0400 & & 0 & 960 & -- & 1.25 & $*$ \\
\hline Do. & 0820 & & 0 & 1,220 & --- & 2.30 & $\star$ \\
\hline Do. & $1028^{1}$ & $\begin{array}{l}120-1 / \\
124-\end{array}$ & 0 & 1,348 & 227 & 2.8 & $*$ \\
\hline Do. & $1152^{1}$ & $\begin{array}{c}124-1 / \\
128^{-}\end{array}$ & 0 & 1,432 & 227 & 2.4 & $*$ \\
\hline
\end{tabular}


Table 56.--Data obtained from the tracer experiments conducted during August 8-15, 1978, at the Stanton, Tex. site--Continued

\begin{tabular}{|c|c|c|c|c|c|c|}
\hline \multicolumn{7}{|c|}{15 -Meter we11 } \\
\hline Date & Clock time & $\begin{array}{l}\text { Elapsed } \\
\text { (d) }\end{array}$ & $\begin{array}{l}\text { time } \\
\text { (min) }\end{array}$ & $\begin{array}{l}\text { Boron } \\
(\mu \mathrm{g} / \mathrm{L})\end{array}$ & $\begin{array}{c}\text { Bromide } \\
(\mathrm{mg} / \mathrm{L})\end{array}$ & $\begin{array}{l}\mathrm{CBrC} \mathrm{F}_{2} \\
(\mathrm{mg} / \mathrm{L})\end{array}$ \\
\hline $8-8-78$ & First & tracer & experiment & started-- & ntinued & \\
\hline $8-9-78$ & 1232 & 1 & 32 & 175 & --- & $*$ \\
\hline Do. & 1348 & 1 & 108 & 126 & $\cdots$ & $*$ \\
\hline Do. & $1420 \begin{array}{c}116-1 \\
120\end{array}$ & 1 & 140 & 116 & 1.8 & $*$ \\
\hline Do. & $1505^{120-1 /}$ & 1 & 185 & 251 & 3.2 & $*$ \\
\hline Do. & $1521 \begin{array}{c}124-1 / \\
128\end{array}$ & 1 & 201 & -- & 3.0 & $*$ \\
\hline Do. & $1540^{128-1} 132-$ & 1 & 220 & -- & 1.6 & $*$ \\
\hline Do. & $1800^{128-1 /}$ & 1 & 480 & 180 & 2.3 & $*$ \\
\hline Do . & 2400 & 1 & 720 & --- & 2.7 & $*$ \\
\hline $8-10-78$ & 0613 & 1 & 1,093 & --- & 2.65 & $*$ \\
\hline Do. & 0815 & 1 & 1,215 & -- & 2.65 & $*$ \\
\hline Do. & $1025^{120-1 /}$ & 1 & 1,345 & 217 & 2.3 & $*$ \\
\hline Do. & $1055^{124-1 /}$ & 1 & 1,375 & -- & 2.9 & $*$ \\
\hline Do. & $1113^{128-1} 132-$ & 1 & 1,393 & 238 & 2.4 & $*$ \\
\hline $8-10-78$ & Seconc & ad tracer & experiment & $t$ started & & \\
\hline $8-10-78$ & 1300 & 2 & 60 & -- & 2.5 & $*$ \\
\hline Do. & 1400 & 2 & 120 & -- & $\cdots$ & $*$ \\
\hline Do. & 1500 & 2 & 180 & -- & ---- & $*$ \\
\hline Do. & 1615 & 2 & 255 & 281 & --- & $\star$ \\
\hline Do. & 1700 & 2 & 300 & --- & 2.3 & $*$ \\
\hline Do. & 1805 & 2 & 265 & -- & $-\cdots$ & $*$ \\
\hline Do. & 1900 & 2 & 420 & -- & --- & $*$ \\
\hline
\end{tabular}


Table 56.--Data obtained from the tracer experiments conducted during August 8-15, 1978, at the Stanton, Tex. site--Continued

\begin{tabular}{|c|c|c|c|c|c|c|}
\hline \multicolumn{7}{|c|}{ 15-Meter wel1 } \\
\hline Date & Clock time & $\begin{array}{l}\text { Elapsed } \\
\text { (d) }\end{array}$ & $\begin{array}{l}\text { time } \\
\text { (min) }\end{array}$ & $\begin{array}{l}\text { Boron } \\
(\mu \mathrm{g} / \mathrm{L})\end{array}$ & $\begin{array}{c}\text { Bromide } \\
(\mathrm{mg} / \mathrm{L})\end{array}$ & $\begin{array}{c}\mathrm{CBrClF}_{2} \\
(\mathrm{mg} / \mathrm{L})\end{array}$ \\
\hline $8-10-78$ & 1300 & Second tracer & experiment & $t$ startec & ontinued & \\
\hline $8-10-78$ & 2000 & 2 & 480 & 267 & --- & * \\
\hline Do. & 2115 & 2 & 555 & -- & --- & $*$ \\
\hline Do. & 2210 & 2 & 610 & -- & 2.05 & $*$ \\
\hline $8-11-78$ & 0004 & 2 & 724 & 172 & --- & $*$ \\
\hline Do. & 0100 & 2 & 780 & -- & 2.1 & $*$ \\
\hline Do. & 0215 & 2 & 855 & -- & $\cdots$ & $*$ \\
\hline Do. & 0410 & 2 & 970 & 238 & --- & $*$ \\
\hline Do. & 0600 & 2 & 1,080 & -- & --- & * \\
\hline Do. & 0800 & 2 & 1,200 & 247 & $-\cdots$ & $*$ \\
\hline Do. & 1025 & 2 & 1,345 & -- & --- & $*$ \\
\hline Do. & 1120 & 2 & 1,400 & $-\cdots$ & $\cdots$ & $*$ \\
\hline Do. & 1406 & 3 & 126 & --- & --- & $*$ \\
\hline Do. & 1614 & 3 & 254 & 202 & 2.45 & * \\
\hline Do. & 1815 & 3 & 375 & -- & $-\cdots$ & * \\
\hline Do. & 2100 & 3 & 540 & 206 & $-\cdots$ & * \\
\hline Do. & 2400 & 3 & 720 & 196 & $-\cdots$ & $*$ \\
\hline $8-12-78$ & 0600 & 3 & 1,080 & 180 & $-\cdots$ & $*$ \\
\hline Do. & 1049 & 3 & 1,369 & 172 & 2.6 & $*$ \\
\hline Do. & 1450 & 4 & 170 & -- & 2.3 & $*$ \\
\hline $8-12-78$ & 1710 & Third tracer & exper iment & started & & \\
\hline $8-12-78$ & 1715 & 4 & 315 & 218 & $-\cdots$ & $*$ \\
\hline Do. & 1910 & 4 & 430 & -- & 2.4 & $*$ \\
\hline Do. & 2300 & 4 & 660 & 176 & --- & $*$ \\
\hline $8-13-78$ & 0100 & 4 & 780 & 166 & 2.3 & $*$ \\
\hline Do. & 0300 & 4 & 900 & 166 & 2.5 & $*$ \\
\hline
\end{tabular}


Table 56.--Data obtained from the tracer experiments conducted during August 8-15, 1978, at the Stanton, Tex. site--Continued

\begin{tabular}{|c|c|c|c|c|c|c|c|}
\hline \multicolumn{8}{|c|}{ 15-Meter well } \\
\hline Date & Clock time & & $\begin{array}{l}\text { Elapsed } \\
\text { (d) }\end{array}$ & $\begin{array}{l}\text { time } \\
\text { (min) }\end{array}$ & $\begin{array}{l}\text { Boron } \\
(\mu \mathrm{g} / \mathrm{L})\end{array}$ & $\begin{array}{c}\text { Bromide } \\
(\mathrm{mg} / \mathrm{L})\end{array}$ & $\begin{array}{l}\mathrm{CBrClF}_{2} \\
(\mathrm{mg} / \mathrm{L})\end{array}$ \\
\hline $8-12-78$ & 1710 & Third & tracer & experiment & started-- & ntinued & $\because$ \\
\hline $8-13-78$ & 0500 & & 4 & 1,020 & 189 & 2.5 & $*$ \\
\hline Do. & 0600 & & 4 & 1,080 & -- & 2.1 & $*$ \\
\hline Do. & 0700 & & 4 & 1,140 & 236 & 2.0 & $*$ \\
\hline Do. & 0800 & & 4 & 1,200 & -- & 2.1 & $*$ \\
\hline Do. & 0900 & & 4 & 1,260 & 274 & 2.1 & $*$ \\
\hline Do. & 1000 & & 4 & 1,320 & --- & 2.1 & $*$ \\
\hline Do. & 1100 & & 4 & 1,380 & 293 & 2.0 & $*$ \\
\hline Do. & 1200 & & 5 & 0 & --- & 2.4 & $*$ \\
\hline Do. & 1300 & & 5 & 60 & 306 & 2.2 & $*$ \\
\hline Do & 1400 & & 5 & 120 & -- & -- & $*$ \\
\hline Do & 1500 & & 5 & 180 & 320 & 2.3 & $*$ \\
\hline Do. & 1600 & & 5 & 240 & -- & 2.3 & $*$ \\
\hline Do. & 1800 & & 5 & 360 & 316 & 2.3 & $*$ \\
\hline Do & 2008 & & 5 & 488 & 332 & 2.3 & $*$ \\
\hline Do. & 2100 & & 5 & 540 & -- & 2.0 & $*$ \\
\hline Do. & 2300 & & 5 & 660 & 338 & 2.3 & $*$ \\
\hline $8-14-78$ & 0300 & & 5 & 900 & 331 & 2.3 & $*$ \\
\hline Do. & 0610 & & 5 & 1,090 & 338 & 2.3 & $*$ \\
\hline Do. & 1002 & & 5 & 1,322 & -- & 2.3 & $*$ \\
\hline $8-14-78$ & 1200 & Fourth & tracer & experiment & started & & \\
\hline $8-14-78$ & 1250 & & 6 & 50 & 330 & 2.4 & $*$ \\
\hline Do. & 1300 & & 6 & 60 & 339 & -- & * \\
\hline Do. & 1400 & & 6 & 120 & 330 & 1.3 & * \\
\hline Do. & 1600 & & 6 & 240 & --- & -- & $*$ \\
\hline
\end{tabular}


Table 56.--Data obtained from the tracer experiments conducted during August 8-15, 1978, at the Stanton, Tex. site--Continued

\begin{tabular}{|c|c|c|c|c|c|c|}
\hline \multicolumn{7}{|c|}{ 15-Meter wel1 } \\
\hline Date & Clock time & $\begin{array}{l}\text { Elapsed } \\
\text { (d) }\end{array}$ & $\begin{array}{l}\text { time } \\
\text { (min) }\end{array}$ & $\begin{array}{l}\text { Boron } \\
(\mu \mathrm{g} / \mathrm{L})\end{array}$ & $\begin{array}{c}\text { Bromide } \\
(\mathrm{mg} / \mathrm{L})\end{array}$ & $\begin{array}{l}\mathrm{CBrClF}_{2} \\
(\mathrm{mg} / \mathrm{L})\end{array}$ \\
\hline $8-14-78$ & 1200 & Fourth tracer & experiment & starte & ontinued & \\
\hline $8-14-78$ & 1800 & 6 & 360 & 320 & 3.7 & $*$ \\
\hline Do. & 2013 & 6 & 493 & -- & --- & $*$ \\
\hline Do. & 2400 & 6 & 720 & 310 & 1.3 & $*$ \\
\hline $8-15-78$ & 0200 & 6 & 840 & -- & -- & $*$ \\
\hline Do. & 0400 & 6 & 960 & 300 & 1.0 & $*$ \\
\hline Do. & 1007 & 6 & 1,327 & 280 & 2.5 & $*$ \\
\hline Do. & 1225 & 7 & 25 & -- & -- & $*$ \\
\hline Do. & 1400 & 7 & 120 & 270 & 3.0 & $*$ \\
\hline Do. & 1800 & 7 & 360 & 270 & 2.2 & $*$ \\
\hline
\end{tabular}


Table 57.--Data obtained from the tracer experiments conducted during August 8-15, 1978, at the Stanton, Tex. site

[Samples are from the observation well $30 \mathrm{~m}$ from the injection we11, which is screened for the saturated thickness of the aquifer. The dash (-) indicates sample is available but was not analyzed; an asterisk (*) indicates the sample was not analyzed and is no longer available.]

\begin{tabular}{|c|c|c|c|c|c|c|}
\hline \multicolumn{7}{|c|}{ 30-Meter well } \\
\hline Date & Clock time & $\begin{array}{l}\text { Elapsed } \\
\text { (d) }\end{array}$ & $\begin{array}{l}\text { time } \\
\text { (min) }\end{array}$ & $\begin{array}{l}\text { Boron } \\
(\mu \mathrm{g} / \mathrm{L})\end{array}$ & $\begin{array}{c}\text { Bromide } \\
(\mathrm{mg} / \mathrm{L})\end{array}$ & $\begin{array}{l}\mathrm{CBrClF}_{2} \\
(\mathrm{mg} / \mathrm{L})\end{array}$ \\
\hline $8-9-78$ & 1400 & 1 & 120 & 127 & -- & $*$ \\
\hline Do. & 1800 & 1 & 360 & 124 & -- & $*$ \\
\hline Do. & 2130 & 1 & 570 & 127 & -- & $*$ \\
\hline $8-10-78$ & 1300 & 2 & 60 & 138 & --- & $*$ \\
\hline Do. & 1500 & 2 & 180 & 136 & -- & $\star$ \\
\hline Do. & 1900 & 2 & 420 & 136 & -- & $*$ \\
\hline Do. & 2210 & 2 & 610 & 136 & -- & $*$ \\
\hline Do. & 2400 & 2 & 720 & 136 & -- & $*$ \\
\hline $8-11-78$ & 0220 & 2 & 860 & 133 & -- & $*$ \\
\hline Do. & 0410 & 2 & 990 & 131 & -- & $*$ \\
\hline Do. & 1015 & 2 & 1,335 & 142 & -- & $*$ \\
\hline Do. & 1202 & 3 & 2 & 144 & -- & * \\
\hline Do. & 1400 & 3 & 128 & 144 & -- & $*$ \\
\hline Do. & 1555 & 3 & 235 & 142 & -- & $*$ \\
\hline Do. & 1805 & 3 & 365 & 147 & -- & $*$ \\
\hline Do. & 2115 & 3 & 555 & 145 & -- & $*$ \\
\hline $8-12-78$ & 0300 & 3 & 900 & 162 & -- & $\star$ \\
\hline Do. & 0600 & 3 & 1,080 & 92 & -- & $*$ \\
\hline Do. & 1045 & 3 & 1,365 & 158 & --- & $*$ \\
\hline Do. & 1700 & 4 & 300 & 160 & --- & $*$ \\
\hline Do. & 1902 & 4 & 422 & 160 & --- & $*$ \\
\hline Do. & 2100 & 4 & 540 & 160 & --- & $*$ \\
\hline Do. & 2300 & 4 & 660 & 156 & -- & $*$ \\
\hline
\end{tabular}


Table 57.--Data obtained from the tracer experiments conducted during August 8-15, 1978, at the Stanton, Tex. site--Continued

\begin{tabular}{|c|c|c|c|c|c|c|}
\hline \multicolumn{7}{|c|}{ 30-Meter wel1 } \\
\hline Date & Clock time & $\begin{array}{l}\text { Elapsed } \\
\text { (d) }\end{array}$ & $\begin{array}{l}\text { time } \\
\text { (min) }\end{array}$ & $\begin{array}{l}\text { Boron } \\
(\mu g / L)\end{array}$ & $\begin{array}{c}\text { Bromide } \\
(\mathrm{mg} / \mathrm{L})\end{array}$ & $\begin{array}{l}\mathrm{CBrClF}_{2} \\
(\mathrm{mg} / \mathrm{L})\end{array}$ \\
\hline $8-13-78$ & 0100 & 4 & 780 & 160 & -- & $*$ \\
\hline Do. & 0500 & 4 & 1,020 & 162 & -- & $*$ \\
\hline Do. & 0700 & 4 & 1,140 & 173 & --- & $*$ \\
\hline Do & 1000 & 4 & 1,320 & 164 & -- & $*$ \\
\hline Do. & 1200 & 5 & 0 & 167 & -- & $*$ \\
\hline Do. & 1400 & 5 & 120 & 176 & --- & $*$ \\
\hline Do. & 1600 & 5 & 240 & 162 & -- & * \\
\hline Do. & 1800 & 5 & 360 & 167 & -- & $*$ \\
\hline Do. & 2001 & 5 & 601 & 166 & -- & $*$ \\
\hline Do. & 2300 & 5 & 660 & 176 & -- & $*$ \\
\hline $8-14-78$ & 0300 & 5 & 900 & 173 & -- & * \\
\hline Do. & 0605 & 5 & 1,085 & 176 & --- & $*$ \\
\hline Do. & 1300 & 6 & 60 & 184 & --- & $*$ \\
\hline
\end{tabular}


Table 58.--Data obtained from the tracer experiments conducted during August 8-15, 1978, at the Stanton, Tex. site

[Samples are from the point sampler designated 30-m black, located $42.4 \mathrm{~m}$ below land surface and $30 \mathrm{~m}$ radially from the injection well. The dash (-) indicates sample is available but was not analyzed; an asterisk (*) indicates the sample was not analyzed and is no longer available.]

\begin{tabular}{|c|c|c|c|c|c|c|}
\hline \multicolumn{7}{|c|}{ 30-Meter black point sampler } \\
\hline Date & Clock time & $\begin{array}{l}\text { Elapsed } \\
\text { (d) }\end{array}$ & $\begin{array}{l}\text { time } \\
\text { (min) }\end{array}$ & $\begin{array}{l}\text { Boron } \\
(\mu g / L)\end{array}$ & $\begin{array}{c}\text { Bromide } \\
(\mathrm{mg} / \mathrm{L})\end{array}$ & $\begin{array}{c}\mathrm{CBrClF}_{2} \\
(\mathrm{mg} / \mathrm{L})\end{array}$ \\
\hline $8-8-78$ & 1800 & --- & 360 & 130 & --- & $*$ \\
\hline Do & 2200 & --- & 600 & 130 & --- & $*$ \\
\hline $8-9-78$ & 0613 & --- & 1,093 & 130 & -- & $*$ \\
\hline Do. & 1200 & 1 & 0 & 140 & --- & $*$ \\
\hline $8-11-78$ & 1200 & 3 & 0 & 150 & --- & $*$ \\
\hline Do. & 1800 & 3 & 360 & 140 & --- & $*$ \\
\hline $8-12-78$ & 1600 & 4 & 240 & 150 & -- & $*$ \\
\hline $8-13-78$ & 1600 & 5 & 240 & 150 & --- & $*$ \\
\hline $8-14-78$ & 0600 & 5 & 1,080 & 170 & --- & * \\
\hline
\end{tabular}


Table 59.--Data obtained from the tracer experiments conducted during August 8-15, 1978, at the Stanton, Tex. site

[Samples are from the point sampler designated $30-\mathrm{m}$ red, located $37.4 \mathrm{~m}$ below land surface and $30 \mathrm{~m}$ radially from the injection well. The dash (-) indicates sample is available but was not analyzed; an asterisk (*) indicates the sample was not analyzed and is no longer available.]

\begin{tabular}{|c|c|c|c|c|c|c|}
\hline \multicolumn{7}{|c|}{ 30-Meter red point sampler } \\
\hline Date & Clock time & $\begin{array}{l}\text { Elapsed } \\
\text { (d) }\end{array}$ & $\begin{array}{l}\text { time } \\
(\min )\end{array}$ & $\begin{array}{l}\text { Boron } \\
(\mu \mathrm{g} / \mathrm{L})\end{array}$ & $\begin{array}{c}\text { Bromide } \\
(\mathrm{mg} / \mathrm{L})\end{array}$ & $\begin{array}{l}\mathrm{CBrClF}_{2} \\
(\mathrm{mg} / \mathrm{L})\end{array}$ \\
\hline $8-8-78$ & 1200 & -- & 0 & 180 & --- & $*$ \\
\hline $8-9-78$ & 0200 & -- & 840 & 220 & --- & $*$ \\
\hline $8-10-78$ & 1500 & 2 & 180 & 180 & -- & $*$ \\
\hline Do. & 1900 & 2 & 420 & 180 & --- & $*$ \\
\hline $8-11-78$ & 1005 & 2 & 1,325 & 180 & -- & $*$ \\
\hline Do. & 2130 & 3 & 540 & 150 & -- & $*$ \\
\hline $8-12-78$ & 1040 & 3 & 1,360 & 170 & -- & $\star$ \\
\hline Do. & 2000 & 4 & 480 & 220 & --- & $*$ \\
\hline $8-13-78$ & 0300 & 4 & 900 & 240 & -- & $*$ \\
\hline Do. & 1200 & 5 & 0 & 190 & -- & $*$ \\
\hline $8-14-78$ & 0600 & 5 & 1,080 & 200 & --- & $*$ \\
\hline
\end{tabular}


Table 60.--Data obtained from the tracer experiments conducted during August 8-15, 1978, at the Stanton, Tex. site

[Samples are from the point sampler designated $30-\mathrm{m}$ orange, located $35.7 \mathrm{~m}$ below land surface and $30 \mathrm{~m}$ radially from the injection well. The dash (-) indicates sample is available but was not analyzed; an asterisk (*) indicates the sample was not analyzed and is no longer available.]

\begin{tabular}{|c|c|c|c|c|c|c|}
\hline \multicolumn{7}{|c|}{ 30-Meter orange point sampler } \\
\hline Date & Clock time & $\begin{array}{l}\text { Elapsed } \\
\text { (d) }\end{array}$ & $\begin{array}{l}\text { time } \\
\text { (min) }\end{array}$ & $\begin{array}{l}\text { Boron } \\
(\mu \mathrm{g} / \mathrm{L})\end{array}$ & $\begin{array}{c}\text { Bromide } \\
(\mathrm{mg} / \mathrm{L})\end{array}$ & $\begin{array}{l}\mathrm{CBrClF}_{2} \\
(\mathrm{mg} / \mathrm{L})\end{array}$ \\
\hline $8-8-78$ & 2000 & --- & 240 & 200 & --- & $*$ \\
\hline $8-9-78$ & 0200 & --- & 840 & 240 & --- & $*$ \\
\hline $8-10-78$ & 1300 & 2 & 60 & 180 & -- & * \\
\hline Do. & 2200 & 2 & 600 & 180 & --- & $*$ \\
\hline $8-11-78$ & 1005 & 2 & 1,325 & 190 & -- & $*$ \\
\hline Do. & 1800 & 3 & 360 & 180 & -- & $*$ \\
\hline $8-12-78$ & 1650 & 4 & 290 & 180 & -- & $*$ \\
\hline Do. & 2400 & 4 & 720 & 170 & --- & $*$ \\
\hline $8-13-78$ & 1600 & 5 & 240 & 240 & -- & $*$ \\
\hline $8-14-78$ & 0600 & 5 & 1,090 & 300 & --- & $*$ \\
\hline
\end{tabular}


at any sampling point to the initial input concentration (Co), the data are presented as a unitless ratio, C/Co (tables 61 through 74). Data normalized in this form facilitate a comparison between the tracers.

The fourth experiment of the August test involved the addition of fluoride, chloride, bromide, iodide, yeast, and three fluorocarbons as a 2-min pulse beginning at 1200 on August 14 using a bypass chamber containing $355 \mathrm{~L}$ of the tracer solution. The concentrations of the three fluorocarbons $\left(\mathrm{F}-12, \mathrm{~F}-11\right.$, and $\left.\mathrm{CBrC}_{1} \mathrm{~F}_{2}\right)$ were too sma11 to detect in the water samples for the fourth experiment, so these compounds were added in an identical manner beginning at 1000 on August 15 for the fifth experiment. The combined results of the fourth and fifth experiments are presented in tables 75 through 82 as unitless concentrations (C/Co). Background concentrations have not been subtracted from the measured concentrations. However, background concentrations of inorganic compounds in the ground water are listed in table 46. Residual concentrations of bromide and $\mathrm{CBrCl}_{2} \mathrm{~F}$ remaining from previous tracer experiments are listed in tables 47 through 60 .

Because the breakthrough of the tracer at the sampling points tended to trend uniformly with time, only samples necessary to describe the trend were analyzed. Many samples are available for future analysis if necessary, and these samples are designated with a $(-)$ in the tables. Selected data from tables 47 to 82 are presented in figures 19 to 38 , as the variation of the tracer concentration with time is more readily seen from graphic data than from tabular data.

\section{DECEMBER 1978 TRACER TEST}

Two-we11 tracer tests are conducted by pumping water from one we11 and injecting it into a nearby we11, with tracer being added after the flow field has stabilized. A two-well tracer test was conducted at the Stanton site by injecting water from well 1 into the injection well to provide a comparison with results obtained during the single-well injection tests conducted during March, May, and August to see whether the two types of tests give similar results.

For the test, injection was started 1330 on December 7,1978 at a rate of $3.8 \mathrm{~L} / \mathrm{s}$. Tracer injection was started 0900 on December 12 , and was halted at 2100 the same day. Injection of ground water continued at the $3.8-\mathrm{L} / \mathrm{s}$ rate until 0900 on December 14, when the well began to flow. The rate was decreased to $3.0 \mathrm{~L} / \mathrm{s}$ until 0900 December 15, when injection was halted. Injection rates and cumulative volumes of injected water are listed in table 83. Flow-meter logs were not obtained for this test. Water levels were measured daily, but are not listed.

We11 1 is equipped with a 22.5-kW electric motor, and it was necessary to greatly constrict a valve in the injection line to maintain sufficient back pressure, so the pump did not break suction. The water apparent1y 
Table 61.--A comparison between alz tracers used during the second tracer experiment written in terms of the unitless concentration ratio $\mathrm{C} / \mathrm{Co}$. The tracers were added simultaneously at 1300, August 10, 1978, and the initial concentrations are given in table 45

[Samples are from the observation well located $2 \mathrm{~m}$ from the injection well. The 2-m we11 is screened for the full-saturated thickness of the aquifer. The dash (-) indicates sample is available but was not analyzed; an asterisk (*) indicates the sample was not analyzed and is no longer available.]

\section{2-Meter we11}

\begin{tabular}{|c|c|c|c|c|c|c|}
\hline Date & $\begin{array}{l}\text { Clock } \\
\text { time }\end{array}$ & $\begin{array}{l}\text { Elapsed } \\
\text { time } \\
\text { (min) }\end{array}$ & Bromide & $\begin{array}{l}\text { Phenol- } \\
\text { phthalein }\end{array}$ & $\begin{array}{l}\text { Benzo- } \\
\text { ate }\end{array}$ & Freon-12 \\
\hline $8-10-78$ & 1310 & 10 & ---- & 0 & 0 & $*$ \\
\hline Do. & 1320 & 20 & --- & 0 & 0 & $*$ \\
\hline Do. & 1330 & 30 & 0.032 & 0 & 0 & 0 \\
\hline Do. & 1340 & 40 & $-\cdots$ & 0 & 0 & $*$ \\
\hline Do. & 1350 & 50 & ---- & 0 & 0 & $\star$ \\
\hline Do. & 1400 & 60 & .038 & 0 & .0091 & 0 \\
\hline Do. & 1410 & 70 & ---- & 0 & .018 & $*$ \\
\hline Do. & 1420 & 80 & $-\cdots$ & $*$ & .027 & 0 \\
\hline Do. & 1430 & 90 & .067 & .07 & .055 & $\star$ \\
\hline Do. & 1445 & 105 & $-\cdots-$ & .11 & .082 & 0 \\
\hline Do. & 1500 & 120 & .125 & .16 & $*$ & .01 \\
\hline Do. & 1515 & 135 & ---- & $*$ & .140 & .02 \\
\hline Do. & 1530 & 150 & .190 & .18 & $*$ & * \\
\hline Do. & 1545 & 165 & .244 & .20 & .210 & .04 \\
\hline Do. & 1600 & 180 & ---- & .22 & $*$ & .05 \\
\hline Do. & 1620 & 200 & ---- & $*$ & $*$ & $*$ \\
\hline Do. & 1630 & 210 & .244 & .27 & .240 & .04 \\
\hline Do. & 1700 & 240 & .260 & .32 & .350 & .10 \\
\hline Do. & 1730 & 270 & .250 & $*$ & .300 & .10 \\
\hline Do. & 1800 & 300 & .230 & .34 & .250 & .10 \\
\hline Do. & 1900 & 360 & ---- & .37 & .160 & .13 \\
\hline Do. & 2000 & 420 & .210 & .34 & .100 & .19 \\
\hline
\end{tabular}


Table 61.--A comparison between all tracers used during the second tracer experiment written in terms of the unitzess concentration ratio $\mathrm{C} / \mathrm{CO}$. The tracers were added simultaneously at 1300, August 10, 1978, and the initial concentrations are given in table 45--Continued

2-Meter well

\begin{tabular}{|c|c|c|c|c|c|c|}
\hline Date & $\begin{array}{l}\text { Clock } \\
\text { time }\end{array}$ & $\begin{array}{c}\text { Elapsed } \\
\text { time } \\
\text { (min) }\end{array}$ & Bromide & $\begin{array}{c}\text { Phenol- } \\
\text { phthalein }\end{array}$ & $\begin{array}{l}\text { Benzo- } \\
\text { ate }\end{array}$ & Freon-12 \\
\hline $8-10-78$ & 2100 & 480 & ----- & 0.25 & $*$ & 0.19 \\
\hline Do. & 2200 & 540 & ---- & .20 & $*$ & .61 \\
\hline Do. & 2300 & 600 & ----- & .20 & $*$ & .17 \\
\hline Do. & 2400 & 660 & ---- & .20 & 0.023 & .16 \\
\hline $8-11-78$ & 0100 & 720 & $-\cdots$ & .18 & $*$ & .15 \\
\hline Do. & 0200 & 780 & $-\cdots--$ & $*$ & 0 & $*$ \\
\hline Do. & 0300 & 840 & ---- & $*$ & $*$ & $*$ \\
\hline Do. & 0400 & 900 & --- & $*$ & $*$ & $*$ \\
\hline Do. & 0600 & 1,020 & ---- & .12 & $*$ & $*$ \\
\hline Do. & 0700 & 1,080 & ---- & $*$ & $*$ & $*$ \\
\hline Do. & 0800 & 1,140 & ---- & .07 & $*$ & $*$ \\
\hline
\end{tabular}


Table 62.--A comparison between all tracers used during the second tracer experiment written in terms of the unitzess concentration ratio $\mathrm{C} / \mathrm{Co}$.

The tracers were added simultaneously at 1300, August 10, 1978, and the initial concentrations are given in table 45

[Samples are from the point sampler designated 2-m red located $2 \mathrm{~m}$ radially from the injection well and $38.7 \mathrm{~m}$ below land surface. The dash (-) indicates sample is available but was not analyzed; an asterisk (*) indicates the sample was not analyzed and is no longer available.]

\begin{tabular}{|c|c|c|c|c|c|c|}
\hline \multicolumn{7}{|c|}{ 2-Meter red point sampler } \\
\hline Date & $\begin{array}{l}\text { Clock } \\
\text { time }\end{array}$ & $\begin{array}{l}\text { Elapsed } \\
\text { time } \\
\text { (min) }\end{array}$ & Bromide & $\begin{array}{c}\text { Phenol- } \\
\text { phthalein }\end{array}$ & $\begin{array}{l}\text { Benzo- } \\
\text { ate }\end{array}$ & Freon-12 \\
\hline $8-10-78$ & 1310 & 10 & ---- & 0 & 0 & $*$ \\
\hline Do. & 1320 & 20 & --- & 0 & 0 & $*$ \\
\hline Do. & 1330 & 30 & 0.150 & 0 & 0 & 0 \\
\hline Do . & 1340 & 40 & ---- & 0 & 0 & $*$ \\
\hline Do. & 1350 & 50 & --- & 0 & 0 & $*$ \\
\hline Do. & 1400 & 60 & ---- & 0 & 0 & 0 \\
\hline Do. & 1410 & 70 & .16 & 0 & 0 & $*$ \\
\hline Do & 1420 & 80 & - - & 0 & 0 & 0 \\
\hline Do. & 1430 & 90 & .13 & 0 & 0 & 0 \\
\hline Do. & 1445 & 105 & -- & 0 & 0 & $*$ \\
\hline Do. & 1500 & 120 & .18 & 0 & 0 & 0 \\
\hline Do. & 1515 & 135 & .16 & 0 & 0 & 0 \\
\hline Do. & 1530 & 150 & ---- & 0 & 0 & $*$ \\
\hline Do. & 1545 & 165 & ---- & 0 & 0 & .04 \\
\hline Do. & 1600 & 180 & ---- & 0 & 0 & 0 \\
\hline Do. & 1620 & 200 & ---- & 0 & 0 & $*$ \\
\hline Do. & 1630 & 210 & - - & 0 & 0 & 0 \\
\hline Do. & 1700 & 240 & .22 & 0 & 0 & .05 \\
\hline Do. & 1730 & 270 & .31 & 0 & .036 & .23 \\
\hline Do. & 1800 & 300 & $\cdots$ & 0 & $*$ & 0 \\
\hline Do. & 1900 & 360 & .48 & 0 & .150 & .85 \\
\hline
\end{tabular}


Table 62.--A comparison between all tracers used during the second tracer experiment written in terms of the unitless concentration ratio $\mathrm{C} / \mathrm{Co}$. The tracers were added simultaneously at 1300, August 10, 1978, and the initial concentrations are given in table 45--Continued

\begin{tabular}{|c|c|c|c|c|c|c|}
\hline \multicolumn{7}{|c|}{ 2-Meter red point sampler } \\
\hline Date & $\begin{array}{r}\text { Clock } \\
\text { time }\end{array}$ & $\begin{array}{c}\text { Elapsed } \\
\text { time } \\
\text { (min) }\end{array}$ & Bromide & $\begin{array}{c}\text { Phenol- } \\
\text { phthalein }\end{array}$ & $\begin{array}{l}\text { Benzo- } \\
\text { ate }\end{array}$ & Freon-12 \\
\hline $8-10-78$ & 2000 & 420 & $-\cdots$ & 0 & $*$ & $*$ \\
\hline Do. & 2100 & 480 & 0.71 & 0 & .250 & 0.39 \\
\hline Do. & 2200 & 540 & .70 & 0 & .270 & 0 \\
\hline Do $\cdot$ & 2300 & 600 & .61 & 0 & $*$ & $*$ \\
\hline Do. & 2400 & 660 & $-\cdots$ & 0 & .120 & 1.80 \\
\hline $8-11-78$ & 0100 & 720 & $-\cdots$ & 0 & $*$ & 1.40 \\
\hline Do. & 0200 & 780 & .47 & 0 & .055 & .70 \\
\hline Do. & 0300 & 840 & .45 & 0 & .13 & 1.80 \\
\hline Do. & 0400 & 900 & $-\cdots$ & 0 & $*$ & $*$ \\
\hline Do & 0600 & 1,020 & ---- & 0 & $*$ & .45 \\
\hline Do. & 0700 & 1,080 & - - & 0 & .009 & $*$ \\
\hline Do. & 0800 & 1,140 & .28 & 0 & 0 & .90 \\
\hline
\end{tabular}


Table 63.--A comparison between all tracers used during the second tracer experiment written in terms of the unitzess concentration ratio $\mathrm{C} / \mathrm{Co}$. The tracers were added simultaneously at 1300, August 10, 1978, and the initial concentrations are given in table 45

[Samples are from the point sampler designated 2-m orange located $34.8 \mathrm{~m}$ below land surface and $2 \mathrm{~m}$ radially from the injection well. The dash (-) indicates sample is available but was not analyzed; an asterisk (*) indicates this sample was not analyzed and is no longer available.]

\begin{tabular}{|c|c|c|c|c|c|c|}
\hline \multicolumn{7}{|c|}{ 2-Meter orange point sampler } \\
\hline Date & $\begin{array}{l}\text { Clock } \\
\text { time }\end{array}$ & $\begin{array}{c}\text { Elapsed } \\
\text { time } \\
\text { (min) }\end{array}$ & Bromide & $\begin{array}{l}\text { Phenol- } \\
\text { phthalein }\end{array}$ & $\begin{array}{l}\text { Benzo- } \\
\text { ate }\end{array}$ & Freon-12 \\
\hline $8-10-78$ & 1310 & 10 & 0.13 & 0 & 0 & $*$ \\
\hline Do. & 1320 & 20 & --- & 0 & 0 & $*$ \\
\hline Do . & 1330 & 30 & .13 & 0 & 0 & $*$ \\
\hline Do. & 1340 & 40 & ---- & 0 & 0 & * \\
\hline Do. & 1350 & 50 & --- & 0 & 0 & * \\
\hline Do. & 1400 & 60 & .27 & 0 & 0 & $*$ \\
\hline Do . & 1410 & 70 & --- & .1 & .045 & $*$ \\
\hline Do. & 1420 & 80 & --- & .13 & .110 & $*$ \\
\hline Do. & 1430 & 90 & .60 & .16 & .160 & $*$ \\
\hline Do. & 1445 & 105 & --- & .2 & .170 & $*$ \\
\hline Do. & 1500 & 120 & .71 & .21 & .160 & $*$ \\
\hline Do. & 1515 & 135 & --- & .25 & .270 & $*$ \\
\hline Do. & 1530 & 150 & .46 & .25 & .450 & $*$ \\
\hline Do. & 1545 & 165 & --- & .3 & .400 & $*$ \\
\hline Do. & 1600 & 180 & .69 & .32 & .350 & $*$ \\
\hline Do. & 1620 & 200 & --- & $*$ & $*$ & $*$ \\
\hline Do. & 1630 & 210 & .69 & .32 & $*$ & $*$ \\
\hline Do. & 1700 & 240 & .92 & .34 & .250 & $*$ \\
\hline Do. & 1730 & 270 & 1.0 & $*$ & .410 & $*$ \\
\hline Do. & 1800 & 300 & .95 & .32 & .170 & $*$ \\
\hline
\end{tabular}


Table 63.--A comparison between all tracers used during the second tracer experiment written in terms of the unitless concentration ratio $\mathrm{C} / \mathrm{Co}$. The tracers were added simultaneously at 1300, August 10, 1978, and the initial concentrations are given in table 45--Continued

\begin{tabular}{|c|c|c|c|c|c|c|}
\hline \multicolumn{7}{|c|}{ 2-Meter orange point sampler } \\
\hline Date & $\begin{array}{l}\text { Clock } \\
\text { time }\end{array}$ & $\begin{array}{c}\text { Elapsed } \\
\text { time } \\
\text { (min) }\end{array}$ & Bromide & $\begin{array}{l}\text { Phenol- } \\
\text { phthalein }\end{array}$ & $\begin{array}{l}\text { Benzo- } \\
\text { ate }\end{array}$ & Freon-12 \\
\hline $8-10-78$ & 1900 & 360 & 0.74 & 0.19 & $*$ & $*$ \\
\hline Do. & 2000 & 420 & .64 & .16 & .091 & $*$ \\
\hline Do. & 2100 & 480 & .40 & .14 & 0 & $*$ \\
\hline Do. & 2200 & 540 & --- & .11 & 0 & $*$ \\
\hline Do. & 2300 & 600 & .25 & .09 & 0 & $*$ \\
\hline Do. & 2400 & 660 & --- & .09 & 0 & $*$ \\
\hline $8-11-78$ & 0100 & 720 & .26 & .11 & 0 & $*$ \\
\hline Do. & 0200 & 780 & ---- & .09 & 0 & * \\
\hline Do. & 0300 & 840 & --- & 0 & 0 & $*$ \\
\hline Do. & 0400 & 900 & ---- & 0 & 0 & $*$ \\
\hline Do. & 0600 & 1,020 & .14 & 0 & 0 & $*$ \\
\hline Do. & 0700 & 1,080 & --- & 0 & 0 & $*$ \\
\hline Do. & 0800 & 1,140 & --- & 0 & 0 & $*$ \\
\hline Do. & 1200 & 1,380 & .14 & 0 & 0 & $*$ \\
\hline
\end{tabular}


Table 64.--A comparison between all tracers used during the second tracer experiment written in terms of the unitzess concentration ratio $\mathrm{C} / \mathrm{Co}$. The tracers were added simultaneously at 1300, August 10, 1978, and the initial concentrations are given in table 45

[Samples are from the observation well located $5 \mathrm{~m}$ from the injection well. The 5-m well is screened for the full-saturated thickness of the aquifer. The dash (-) indicates sample is available but was not analyzed; an asterisk (*) indicates the sample was not analyzed and is no longer available.]

5-Meter we11

\begin{tabular}{|c|c|c|c|c|c|c|}
\hline Date & $\begin{array}{l}\text { Clock } \\
\text { time }\end{array}$ & $\begin{array}{c}\text { Elapsed } \\
\text { time } \\
\text { (min) }\end{array}$ & Bromide & $\begin{array}{l}\text { Phenol- } \\
\text { phthalein }\end{array}$ & $\begin{array}{l}\text { Benzo- } \\
\text { ate }\end{array}$ & Freon-12 \\
\hline $8-10-78$ & 1400 & 60 & 0.06 & 0 & 0 & $*$ \\
\hline Do. & 1410 & 70 & ---- & 0 & .0045 & $*$ \\
\hline Do. & 1430 & 90 & .16 & .07 & .045 & $*$ \\
\hline Do. & 1445 & 105 & --- & .11 & $*$ & 0.01 \\
\hline Do. & 1500 & 120 & .17 & .16 & .12 & .04 \\
\hline Do. & 1515 & 135 & .31 & $*$ & $*$ & .07 \\
\hline Do. & 1530 & 150 & .27 & .2 & .19 & $*$ \\
\hline Do. & 1545 & 165 & --- & .25 & $*$ & .12 \\
\hline Do. & 1600 & 180 & .53 & .25 & .25 & .19 \\
\hline Do. & 1630 & 210 & .42 & .32 & .31 & .15 \\
\hline Do. & 1700 & 240 & --- & .39 & .35 & .39 \\
\hline Do. & 1800 & 300 & .57 & .45 & .29 & .78 \\
\hline Do. & 1900 & 360 & .53 & .39 & .18 & .71 \\
\hline Do. & 2000 & 420 & --- & .25 & .064 & .76 \\
\hline Do. & 2100 & 480 & .31 & .25 & .036 & .73 \\
\hline Do. & 2200 & 540 & .27 & .13 & 0 & .20 \\
\hline Do. & 2300 & 600 & .22 & .13 & 0 & .47 \\
\hline Do. & 2400 & 660 & .17 & .16 & 0 & .47 \\
\hline $8-11-78$ & 0100 & 720 & --- & .16 & 0 & .45 \\
\hline Do. & 0300 & 840 & .15 & 0 & 0 & $*$ \\
\hline
\end{tabular}


Table 64.--A comparison between all tracers used during the second tracer experiment written in terms of the unitless concentration ratio $\mathrm{C} / \mathrm{Co}$. The tracers were added simultaneously at 1300, August 10, 1978, and the initial concentrations are given in table 45--Continued

\begin{tabular}{|c|c|c|c|c|c|c|}
\hline \multicolumn{7}{|c|}{ 5-Meter well } \\
\hline Date & $\begin{array}{l}\text { Clock } \\
\text { time }\end{array}$ & $\begin{array}{c}\text { Elapsed } \\
\text { time } \\
\text { (min) }\end{array}$ & Bromide & $\begin{array}{l}\text { Phenol- } \\
\text { phthalein }\end{array}$ & $\begin{array}{l}\text { Benzo-: } \\
\text { ate }\end{array}$ & Freon-12 \\
\hline $8-11-78$ & 0600 & 1,020 & --- & 0 & 0 & $*$ \\
\hline Do. & 0800 & 1,140 & 0.13 & 0 & 0 & $\star$ \\
\hline Do. & 1000 & 1,260 & ---- & 0 & 0 & 0.10 \\
\hline Do. & 2400 & 2,100 & --- & 0 & 0 & .04 \\
\hline
\end{tabular}


Table 65.--A comparison between all tracers used during the second tracer experiment written in terms of the unitless concentration ratio $\mathrm{C} / \mathrm{Co}$. The tracers were added simultaneously at 1300, August 10, 1978, and the initial concentrations are given in table 45

[Samples are from the point sampler designated 5-m orange located $37.5 \mathrm{~m}$ below land surface and $5 \mathrm{~m}$ radially from the injection well. The dash (-) indicates sample is available but was not analyzed; an asterisk (*) indicates the sample was not analyzed and is no longer available. ]

\begin{tabular}{|c|c|c|c|c|c|c|}
\hline \multicolumn{7}{|c|}{ 5-Meter orange point sampler } \\
\hline Date & $\begin{array}{l}\text { Clock } \\
\text { time }\end{array}$ & $\begin{array}{c}\text { Elapsed } \\
\text { time } \\
\text { (min) }\end{array}$ & Bromide & $\begin{array}{l}\text { Phenol- } \\
\text { phthalein }\end{array}$ & $\begin{array}{l}\text { Benzo- } \\
\text { ate }\end{array}$ & Freon-12 \\
\hline $8-10-78$ & 1400 & 60 & 0.05 & 0 & 0 & 0 \\
\hline Do. & 1410 & 70 & --- & 0 & 0 & $*$ \\
\hline Do. & 1430 & 90 & .05 & 0 & .0091 & 0 \\
\hline Do. & 1445 & 105 & --- & 0 & .018 & $*$ \\
\hline Do. & 1500 & 120 & .14 & .07 & $*$ & 0 \\
\hline Do. & 1515 & 135 & --- & .07 & .064 & 0 \\
\hline Do. & 1530 & 150 & .45 & .07 & .140 & $*$ \\
\hline Do. & 1545 & 165 & $-\cdots$ & .08 & $*$ & * \\
\hline Do. & 1600 & 180 & .43 & .12 & .26 & .63 \\
\hline Do. & 1630 & 210 & .48 & .13 & $*$ & .85 \\
\hline Do. & 1700 & 240 & .58 & .12 & .39 & 1.30 \\
\hline Do. & 1800 & 300 & .63 & .17 & .43 & 2.10 \\
\hline Do. & 1900 & 360 & 1.0 & $*$ & .48 & 3.50 \\
\hline Do. & 2000 & 420 & .79 & .25 & .45 & 1.90 \\
\hline Do. & 2100 & 480 & .69 & .22 & .32 & $\star$ \\
\hline Do. & 2200 & 540 & --- & $*$ & $*$ & 2.30 \\
\hline Do. & 2300 & 600 & .40 & .13 & .11 & $*$ \\
\hline Do. & 2400 & 660 & --- & $*$ & $*$ & $*$ \\
\hline $8-11-78$ & 0100 & 720 & .23 & .10 & .05 & $*$ \\
\hline Do. & 0300 & 840 & .14 & $*$ & .009 & $*$ \\
\hline Do. & 0600 & 1,020 & .08 & .07 & 0 & $*$ \\
\hline Do. & 0800 & 1,140 & ---- & 0 & 0 & $*$ \\
\hline
\end{tabular}


Table 66.--A comparison between all tracers used during the second tracer experiment written in terms of the unitless concentration ratio $\mathrm{C} / \mathrm{Co}$. The tracers were added simultaneously at 1300, August 10, 1978, and the initial concentrations are given in table 45

[Samples are from the observation well located $15 \mathrm{~m}$ from the injection well, which is screened for the saturated thickness of the aquifer. The dash (-) indicates sample is available but was not analyzed; an asterisk (*) indicates the sample was not analyzed and is no longer available.]

15-Meter well

\begin{tabular}{|c|c|c|c|c|c|c|c|}
\hline Date & $\begin{array}{l}\text { Clock } \\
\text { time }\end{array}$ & $\begin{array}{r}E 1 \\
t \\
\text { (d) }\end{array}$ & $\begin{array}{l}\text { apsed } \\
\text { ime } \\
\text { (min) }\end{array}$ & Bromide & $\begin{array}{l}\text { Phenol- } \\
\text { phthalein }\end{array}$ & $\begin{array}{l}\text { Benzo- } \\
\text { ate }\end{array}$ & Freon-12 \\
\hline $8-10-78$ & 1615 & 0 & 195 & --- & 0 & 0 & * \\
\hline Do. & 1700 & 0 & 240 & 0.12 & 0 & 0 & $*$ \\
\hline Do. & 1800 & 0 & 300 & --- & 0 & 0 & $*$ \\
\hline Do. & 1900 & 0 & 360 & --- & 0 & 0 & $*$ \\
\hline Do. & 2000 & 0 & 420 & ---- & 0 & 0 & $*$ \\
\hline Do. & 2200 & 0 & 540 & .10 & 0 & 0 & $*$ \\
\hline Do. & 2400 & 0 & 660 & --- & 0 & .0045 & * \\
\hline $8-11-78$ & 0100 & 0 & 720 & .10 & 0 & $*$ & $*$ \\
\hline Do. & 0200 & 0 & 780 & --- & 0 & .0073 & * \\
\hline Do. & 0215 & 0 & 795 & --- & 0 & $*$ & $*$ \\
\hline Do. & 0300 & 0 & 840 & --- & 0 & .014 & * \\
\hline Do. & 0315 & 0 & 855 & ---- & 0 & $*$ & * \\
\hline Do. & 0400 & 0 & 900 & ---- & 0 & .014 & $*$ \\
\hline Do. & 0410 & 0 & 910 & ---- & 0 & $*$ & * \\
\hline Do. & 0600 & 0 & 1,020 & --- & 0 & .017 & $*$ \\
\hline Do. & 0800 & 0 & 1,140 & --- & 0 & .017 & $*$ \\
\hline Do. & 1030 & 0 & 1,290 & --- & 0 & $*$ & $*$ \\
\hline Do. & 1100 & 0 & 1,320 & --- & 0 & .0091 & $*$ \\
\hline Do. & 1130 & 0 & 1,350 & --- & 0 & $*$ & * \\
\hline Do. & 1400 & 1 & 60 & ---- & 0 & .0045 & $*$ \\
\hline Do. & 1600 & 1 & 180 & .10 & 0 & .001 & $*$ \\
\hline Do. & 1615 & 1 & 195 & --- & 0 & 0 & $*$ \\
\hline Do. & 1815 & 1 & 315 & ---- & 0 & 0 & $*$ \\
\hline Do. & 2100 & 1 & 480 & ---- & 0 & 0 & $*$ \\
\hline
\end{tabular}


Table 67.--A comparison between all tracers used during the third tracer experiment written in terms of the unitless concentration ratio $\mathrm{C} / \mathrm{Co}$. The tracers were added simultaneously at 1710, August 12, 1978, and the initial concentrations are given in table 45

[Samples are from the observation well located $2 \mathrm{~m}$ from the injection well. The 2-m well is screened for the full saturated thickness of the aquifer. The dash (-) indicates sample is available but was not analyzed; an asterisk (*) indicates the sample was not analyzed and is no longer available.]

\begin{tabular}{|c|c|c|c|c|c|c|c|}
\hline \multicolumn{8}{|c|}{ 2-Meter well } \\
\hline \multirow[t]{2}{*}{ Date } & \multirow{2}{*}{$\begin{array}{l}\text { Clock } \\
\text { time }\end{array}$} & \multicolumn{2}{|c|}{$\begin{array}{l}\text { Elapsed } \\
\text { time }\end{array}$} & \multirow[t]{2}{*}{ Boron } & \multirow[t]{2}{*}{ Aniline } & \multirow{2}{*}{$\begin{array}{l}\text { Ethyl- } \\
\text { amine }\end{array}$} & \multirow{2}{*}{$\mathrm{CBr}_{2} \mathrm{~F}_{2}$} \\
\hline & & (d) & (min) & & & & \\
\hline $8-12-78$ & 1710 & 0 & 0 & --- & $*$ & $\star$ & * \\
\hline Do. & 1730 & 0 & 20 & --- & $\star$ & $*$ & $*$ \\
\hline Do. & 1800 & 0 & 50 & 0.10 & 0.005 & 0 & 0.01 \\
\hline Do. & 1830 & 0 & 80 & .10 & .06 & $*$ & .05 \\
\hline Do. & 1900 & 0 & 110 & .13 & $*$ & .022 & .02 \\
\hline Do. & 1930 & 0 & 140 & .15 & .27 & $\star$ & .10 \\
\hline Do. & 2000 & 0 & 170 & .26 & .21 & $*$ & .05 \\
\hline Do. & 2030 & 0 & 200 & --- & .28 & $*$ & .22 \\
\hline Do. & 2100 & 0 & 230 & $-\cdots$ & $*$ & $*$ & .25 \\
\hline Do. & 2130 & 0 & 260 & .31 & .35 & .022 & .21 \\
\hline Do. & 2200 & 0 & 290 & --- & .35 & $*$ & .30 \\
\hline Do. & 2230 & 0 & 320 & .32 & .38 & * & .29 \\
\hline Do. & 2300 & 0 & 350 & .38 & .40 & $*$ & .25 \\
\hline Do. & 2330 & 0 & 380 & .39 & .43 & $*$ & .33 \\
\hline Do. & 2400 & 0 & 410 & .41 & .45 & $*$ & .35 \\
\hline $8-13-78$ & 0030 & 0 & 440 & --- & $*$ & $*$ & .31 \\
\hline Do. & 0100 & 0 & 470 & .38 & .46 & .064 & .37 \\
\hline Do. & 0130 & 0 & 500 & --- & .46 & $*$ & $*$ \\
\hline Do. & 0200 & 0 & 530 & ---- & .46 & $*$ & .43 \\
\hline Do. & 0230 & 0 & 560 & .31 & .43 & .064 & .46 \\
\hline Do. & 0300 & 0 & 590 & --- & .39 & $*$ & .35 \\
\hline
\end{tabular}


Table 67.--A comparison between all tracers used during the third tracer experiment written in terms of the unitzess concentration ratio $\mathrm{C} / \mathrm{Co}$. The tracers were added simultaneously at 1710, August 12, 1978, and the initial concentrations are given in table 45--Continued

\begin{tabular}{|c|c|c|c|c|c|c|c|}
\hline \multicolumn{8}{|c|}{ 2-Meter we11 } \\
\hline \multirow[t]{2}{*}{ Date } & \multirow{2}{*}{$\begin{array}{r}\text { Clock } \\
\text { time }\end{array}$} & \multicolumn{2}{|c|}{$\begin{array}{c}\text { Elapsed } \\
\text { time }\end{array}$} & \multirow[t]{2}{*}{ Boron } & \multirow[t]{2}{*}{ Aniline } & \multirow{2}{*}{$\begin{array}{l}\text { Ethyl- } \\
\text { amine }\end{array}$} & \multirow{2}{*}{$\mathrm{CBr}_{2} \mathrm{~F}_{2}$} \\
\hline & & (d) & (min) & & & & \\
\hline $8-13-78$ & 0330 & 0 & 620 & $-\cdots$ & 0.35 & $*$ & $*$ \\
\hline Do. & 0400 & 0 & 650 & 0.25 & .32 & 0.064 & $*$ \\
\hline Do. & 0500 & 0 & 710 & --- & .25 & .042 & 0.41 \\
\hline Do. & 0600 & 0 & 770 & .20 & .21 & $*$ & .43 \\
\hline Do. & 0700 & 0 & 830 & $-\cdots$ & .18 & .042 & .44 \\
\hline Do. & 0800 & 0 & 890 & .17 & .14 & $*$ & .41 \\
\hline Do. & 0900 & 0 & 950 & --- & .12 & $*$ & .42 \\
\hline Do. & 1000 & 0 & 1,010 & .15 & .10 & .042 & .41 \\
\hline Do. & 1200 & 0 & 1,130 & --- & .09 & $*$ & $*$ \\
\hline Do. & 1400 & 0 & 1,250 & .12 & .06 & $\star$ & * \\
\hline Do. & 1600 & 0 & 1,370 & .10 & .04 & $*$ & $*$ \\
\hline Do. & 1800 & 1 & 50 & --- & $*$ & $*$ & $*$ \\
\hline Do. & 2000 & 1 & 170 & .12 & .03 & .042 & $*$ \\
\hline Do. & 2300 & 1 & 350 & .08 & .02 & * & $*$ \\
\hline
\end{tabular}


Table 68.--A comparison between all tracers used during the third tracer experiment written in terms of the unitless concentration ratio $\mathrm{C} / \mathrm{Co}$. The tracers were added simultaneously at 1710, August 12, 1978, and the initial concentrations are given in table 45

[Samples are from the point sampler designated $2-\mathrm{m}$ red located $2 \mathrm{~m}$ radially from the injection well and $38.7 \mathrm{~m}$ below land surface. The dash (-) indicates sample is available but was not analyzed; an asterisk $(*)$ indicates the sample was not analyzed and is no longer available.]

\begin{tabular}{|c|c|c|c|c|c|c|c|}
\hline & & & leter $r$ & point s & ler & & \\
\hline Date & Clock & $E$ & ed & Boron & Aniline & Ethyl- & $\mathrm{CBr}_{2} \mathrm{~F}_{2}$ \\
\hline & & (d) & (min) & & & & \\
\hline $8-12-78$ & 1700 & 0 & 0 & ---- & 0 & $*$ & $*$ \\
\hline Do. & 1730 & 0 & 20 & 0.03 & 0 & $*$ & $*$ \\
\hline Do. & 1800 & 0 & 50 & .03 & 0 & $*$ & $*$ \\
\hline Do. & 1830 & 0 & 80 & .03 & 0 & $*$ & $*$ \\
\hline Do. & 1900 & 0 & 110 & .03 & 0 & $\star$ & $*$ \\
\hline Do. & 1930 & 0 & 140 & ---- & .005 & $*$ & $*$ \\
\hline Do. & 2000 & 0 & 170 & .04 & .027 & $*$ & $*$ \\
\hline Do. & 2030 & 0 & 200 & --- & $*$ & $*$ & 0.01 \\
\hline Do. & 2100 & 0 & 230 & .06 & .043 & $*$ & $*$ \\
\hline Do. & 2130 & 0 & 260 & ---- & .043 & $*$ & .01 \\
\hline Do. & 2200 & 0 & 290 & .07 & $*$ & $*$ & .01 \\
\hline Do. & 2230 & 0 & 320 & ---- & .085 & 0 & .03 \\
\hline Do. & 2300 & 0 & 350 & .13 & $*$ & $*$ & .05 \\
\hline Do. & 2330 & 0 & 380 & $-\cdots$ & .17 & $*$ & .02 \\
\hline Do. & 2400 & 0 & 410 & .18 & .19 & $*$ & .02 \\
\hline $8-13-78$ & 0030 & 0 & 440 & .22 & .27 & $*$ & .04 \\
\hline Do. & 0100 & 0 & 470 & ---- & .32 & 0 & .01 \\
\hline Do. & 0130 & 0 & 500 & .31 & .37 & $*$ & .01 \\
\hline Do. & 0200 & 0 & 530 & --- & .41 & $*$ & .15 \\
\hline Do. & 0230 & 0 & 560 & ---- & .44 & $*$ & .04 \\
\hline Do. & 0300 & 0 & 590 & ---- & .47 & $*$ & .09 \\
\hline
\end{tabular}


Table 68.--A comparison between all tracers used during the third tracer experiment written in terms of the unitless concentration ratio $\mathrm{C} / \mathrm{Co}$. The tracers were added simultaneously at 1710, August 12, 1978, and the initial concentrations are given in table 45--Continued

\begin{tabular}{|c|c|c|c|c|c|c|c|}
\hline \multicolumn{8}{|c|}{ 2-Meter red point sampler } \\
\hline \multirow[t]{2}{*}{ Date } & \multirow{2}{*}{$\begin{array}{l}\text { Clock } \\
\text { time }\end{array}$} & \multicolumn{2}{|c|}{$\begin{array}{c}\text { Elapsed } \\
\text { time }\end{array}$} & \multirow[t]{2}{*}{ Boron } & \multirow[t]{2}{*}{ Aniline } & \multirow{2}{*}{$\begin{array}{l}\text { Ethy1- } \\
\text { amine }\end{array}$} & \multirow{2}{*}{$\mathrm{CBr}_{2} \mathrm{~F}_{2}$} \\
\hline & & (d) & (min) & & & & \\
\hline $8-13-78$ & 0330 & 0 & 620 & ---- & 0.51 & $*$ & 0.19 \\
\hline Do. & 0400 & 0 & 650 & .40 & .52 & * & .17 \\
\hline Do. & 0500 & 0 & 710 & .39 & .54 & 0.092 & .19 \\
\hline Do. & 0600 & 0 & 770 & ---- & .52 & .019 & .04 \\
\hline Do. & 0700 & 0 & 830 & .35 & .50 & .021 & .06 \\
\hline Do. & 0800 & 0 & 890 & .30 & .41 & $*$ & .25 \\
\hline Do. & 0900 & 0 & 950 & --- & .36 & .015 & .01 \\
\hline Do. & 1000 & 0 & 1,010 & .26 & $*$ & $*$ & .01 \\
\hline Do. & 1200 & 0 & 1,130 & .23 & .29 & $*$ & .12 \\
\hline Do. & 1400 & 0 & 1,250 & .25 & .27 & $*$ & $\star$ \\
\hline Do. & 1600 & 0 & 1,370 & .20 & .15 & .019 & $*$ \\
\hline Do. & 1800 & 1 & 50 & .13 & .10 & $*$ & .35 \\
\hline Do. & 2000 & 1 & 170 & ---- & .07 & \pm & $*$ \\
\hline Do. & 2300 & 1 & 350 & ---- & .06 & $*$ & $*$ \\
\hline $8-14-78$ & 0300 & 1 & 590 & .12 & .05 & * & $*$ \\
\hline Do. & 0400 & 1 & 650 & ---- & $*$ & $*$ & $*$ \\
\hline Do. & 0600 & 1 & 770 & .084 & .04 & $* \quad *$ & $*$ \\
\hline
\end{tabular}


Table 69.--A comparison between all tracers used during the third tracer experiment written in terms of the unitzess concentration ratio $\mathrm{C} / \mathrm{Co}$. The tracers were added simultaneously at 1710, August 12, 1978, and the initial concentrations are given in table 45

[Samples are from the point sampler designated 2-m orange located $34.8 \mathrm{~m}$ below land surface and $2 \mathrm{~m}$ radially from the injection well. The dash $(-)$ indicates sample is available but was not analyzed; an asterisk (*) indicates this sample was not analyzed and is no longer available.]

2-Meter orange point sampler

\begin{tabular}{|c|c|c|c|c|c|c|c|}
\hline \multirow[t]{2}{*}{ Date } & \multirow{2}{*}{$\begin{array}{l}\text { Clock } \\
\text { time }\end{array}$} & \multicolumn{2}{|c|}{ Elapsed } & \multirow[t]{2}{*}{ Boron } & \multirow[t]{2}{*}{ Aniline } & \multirow{2}{*}{$\begin{array}{l}\text { Ethyl- } \\
\text { amine }\end{array}$} & \multirow{2}{*}{$\mathrm{CBr}_{2} \mathrm{~F}_{2}$} \\
\hline & & (d) & (min) & & & & \\
\hline $8-12-78$ & 1710 & 0 & 0 & $-\cdots$ & $*$ & $*$ & $*$ \\
\hline Do. & 1730 & 0 & 20 & --- & 0.01 & $*$ & $*$ \\
\hline Do. & 1800 & 0 & 50 & --- & .043 & 0 & $*$ \\
\hline Do. & 1830 & 0 & 80 & 0.21 & .15 & $*$ & $*$ \\
\hline Do. & 1900 & 0 & 110 & .31 & .27 & .042 & $*$ \\
\hline Do. & 1930 & 0 & 140 & .37 & $*$ & $*$ & $*$ \\
\hline Do. & 2000 & 0 & 170 & --- & $*$ & 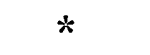 & $*$ \\
\hline Do. & 2030 & 0 & 200 & .42 & .45 & .064 & $*$ \\
\hline Do. & 2100 & 0 & 230 & .49 & .52 & $*$ & $\star$ \\
\hline Do. & 2130 & 0 & 260 & --- & .55 & $*$ & $*$ \\
\hline Do. & 2200 & 0 & 290 & .52 & .56 & $*$ & $*$ \\
\hline Do. & 2230 & 0 & 320 & --- & $*$ & $*$ & $*$ \\
\hline Do. & 2300 & 0 & 350 & .55 & .56 & $*$ & $*$ \\
\hline Do. & 2330 & 0 & 380 & --- & .58 & .127 & $*$ \\
\hline Do. & 2400 & 0 & 410 & $-\cdots$ & .58 & $*$ & $*$ \\
\hline $8-13-78$ & 0030 & 0 & 440 & .51 & $*$ & $*$ & $*$ \\
\hline Do. & 0100 & 0 & 470 & --- & .59 & .170 & $*$ \\
\hline Do. & 0130 & 0 & 500 & $-\cdots$ & .59 & $*$ & $*$ \\
\hline Do. & 0200 & 0 & 530 & .33 & .56 & $*$ & $*$ \\
\hline Do. & 0230 & 0 & 560 & --- & .49 & $*$ & $*$ \\
\hline
\end{tabular}


Table 69.--A comparison between all tracers used during the third tracer experiment written in terms of the unitless concentration ratio $\mathrm{C} / \mathrm{Co}$. The tracers were added simultaneously at 1710, August 12, 1978, and the initial concentrations are given in table 45--Continued

\begin{tabular}{|c|c|c|c|c|c|c|c|}
\hline \multicolumn{8}{|c|}{ 2-Meter orange point sampler } \\
\hline \multirow[t]{2}{*}{ Date } & \multirow{2}{*}{$\begin{array}{r}\text { Clock } \\
\text { time }\end{array}$} & \multicolumn{2}{|c|}{$\begin{array}{c}\text { Elapsed } \\
\text { time }\end{array}$} & \multirow[t]{2}{*}{ Boron } & \multirow[t]{2}{*}{ Aniline } & \multirow{2}{*}{$\begin{array}{l}\text { Ethyl- } \\
\text { amine }\end{array}$} & \multirow{2}{*}{$\mathrm{CBr}_{2} \mathrm{~F}_{2}$} \\
\hline & & (d) & $(\min )$ & & & & \\
\hline $8-13-78$ & 0300 & 0 & 590 & ---- & 0.43 & 0.192 & $*$ \\
\hline Do. & 0330 & 0 & 620 & --- & .35 & $*$ & $*$ \\
\hline Do . & 0400 & 0 & 650 & ---- & .30 & $*$ & $*$ \\
\hline Do. & 0500 & 0 & 710 & 0.19 & .24 & .127 & $*$ \\
\hline Do. & 0600 & 0 & 770 & --- & .24 & $*$ & $*$ \\
\hline Do. & 0700 & 0 & 830 & ---- & .18 & .106 & $*$ \\
\hline Do. & 0800 & 0 & 890 & --- & .16 & $*$ & $*$ \\
\hline Do. & 0900 & 0 & 950 & ---- & .17 & $*$ & $*$ \\
\hline Do. & 1000 & 0 & 1,010 & .14 & .13 & .085 & $*$ \\
\hline Do. & 1200 & 0 & 1,130 & ---- & .12 & $\star$ & $*$ \\
\hline Do. & 1400 & 0 & 1,250 & .22 & .15 & $*$ & $*$ \\
\hline Do. & 1600 & 0 & 1,370 & --- & .13 & $*$ & $*$ \\
\hline Do. & 1800 & 1 & 50 & --- & .10 & .064 & $*$ \\
\hline Do. & 2000 & 1 & 170 & .10 & .07 & $*$ & $*$ \\
\hline Do. & 2300 & 1 & 350 & ---- & .06 & .042 & $*$ \\
\hline $8-14-78$ & 0300 & 1 & 590 & ---- & .06 & $*$ & $*$ \\
\hline Do. & 0400 & 1 & 650 & --- & $*$ & $*$ & $*$ \\
\hline Do. & 0600 & 1 & 770 & --- & .03 & $*$ & $*$ \\
\hline
\end{tabular}


Table 70.--A comparison between all tracers used during the third tracer experiment written in terms of the unitless concentration ratio $\mathrm{C} / \mathrm{Co}$. The tracers were added simultaneously at 1710, August 12, 1978, and the initial concentrations are given in table 45

[Samples are from the observation well located $5 \mathrm{~m}$ from the injection well. The 5-m well is screened for the full-saturated thickness of the aquifer. The dash (-) indicates sample is available but was not analyzed; an asterisk (*) indicates the sample was not analyzed and is no longer available.]

5-Meter we11

\begin{tabular}{|c|c|c|c|c|c|c|c|}
\hline \multirow[t]{2}{*}{ Date } & \multirow{2}{*}{$\begin{array}{l}\text { Clock } \\
\text { time }\end{array}$} & \multicolumn{2}{|c|}{ Elapsed } & \multirow[t]{2}{*}{ Boron } & \multirow[t]{2}{*}{ Aniline } & \multirow{2}{*}{$\begin{array}{l}\text { Ethyl- } \\
\text { amine }\end{array}$} & \multirow{2}{*}{$\mathrm{CBr}_{2} \mathrm{~F}_{2}$} \\
\hline & & (d) & $(\min )$ & & & & \\
\hline $8-12-78$ & 1710 & 0 & 0 & 0.05 & 0 & $*$ & $*$ \\
\hline Do. & 1730 & 0 & 20 & .05 & $*$ & $*$ & 1.0 \\
\hline Do. & 1800 & 0 & 50 & .05 & 0 & 0.006 & $*$ \\
\hline Do. & 1830 & 0 & 80 & .10 & .03 & $*$ & $*$ \\
\hline Do. & 1845 & 0 & 95 & .13 & $*$ & $*$ & $*$ \\
\hline Do. & 1900 & 0 & 110 & --- & .11 & $*$ & .04 \\
\hline Do. & 1930 & 0 & 140 & --- & .18 & .021 & .14 \\
\hline Do. & 1945 & 0 & 155 & .22 & $*$ & $\stackrel{*}{*}$ & $*$ \\
\hline Do. & 2000 & 0 & 170 & --- & .24 & $*$ & .18 \\
\hline Do. & 2030 & 0 & 200 & --- & .28 & $*$ & $*$ \\
\hline Do. & 2100 & 0 & 230 & $-\cdots$ & .33 & $*$ & .37 \\
\hline Do. & 2130 & 0 & 260 & .36 & .36 & .042 & $*$ \\
\hline Do. & 2200 & 0 & 290 & --- & .38 & $*$ & $*$ \\
\hline Do. & 2230 & 0 & 320 & .39 & .39 & $*$ & .49 \\
\hline Do. & 2300 & 0 & 350 & ---- & .40 & $*$ & .46 \\
\hline Do. & 2330 & 0 & 380 & --- & .41 & $*$ & .47 \\
\hline Do. & 2400 & 0 & 410 & .43 & .43 & .064 & .47 \\
\hline $8-13-78$ & 0030 & 0 & 440 & .44 & .44 & $*$ & .52 \\
\hline Do. & 0100 & 0 & 470 & --- & .45 & $*$ & .53 \\
\hline Do. & 0130 & 0 & 500 & $-\cdots$ & .46 & $*$ & .42 \\
\hline Do. & 0200 & 0 & 530 & $-\cdots$ & .47 & .085 & .61 \\
\hline
\end{tabular}


Table 70.--A comparison between all tracers used during the third tracer experiment written in terms of the unitless concentration ratio $\mathrm{C} / \mathrm{Co}$. The tracers were added simultaneously at 1710, August 12, 1978, and the initial concentrations are given in table 45--Continued

\begin{tabular}{|c|c|c|c|c|c|c|c|}
\hline \multicolumn{8}{|c|}{ 5-Meter we11 } \\
\hline \multirow[t]{2}{*}{ Date } & \multirow{2}{*}{$\begin{array}{c}\text { Clock } \\
\text { time }\end{array}$} & \multicolumn{2}{|c|}{$\begin{array}{l}\text { Elapsed } \\
\text { time }\end{array}$} & \multirow[t]{2}{*}{ Boron } & \multirow[t]{2}{*}{ Aniline } & \multirow{2}{*}{$\begin{array}{l}\text { Ethyl- } \\
\text { amine }\end{array}$} & \multirow{2}{*}{$\mathrm{CBr}_{2} \mathrm{~F}_{2}$} \\
\hline & & (d) & (min) & & & & \\
\hline $8-13-78$ & 0230 & 0 & 560 & 0.30 & 0.44 & $*$ & 0.58 \\
\hline Do. & 0300 & 0 & 590 & --- & .38 & $*$ & .53 \\
\hline Do. & 0330 & 0 & 620 & --- & .33 & 0.085 & $*$ \\
\hline Do. & 0400 & 0 & 650 & .23 & .29 & $*$ & .58 \\
\hline Do. & 0500 & 0 & 710 & ---- & .22 & .064 & .42 \\
\hline Do. & 0600 & 0 & 770 & .17 & .17 & .064 & .38 \\
\hline Do. & 0700 & 0 & 830 & --- & .13 & * & .41 \\
\hline Do. & 0800 & 0 & 890 & .14 & .11 & $*$ & .46 \\
\hline Do. & 0900 & 0 & 950 & ---- & .10 & $*$ & .51 \\
\hline Do. & 1000 & 0 & 1,010 & .13 & .08 & .064 & .47 \\
\hline Do. & 1200 & 0 & 1,130 & .11 & .05 & $*$ & .43 \\
\hline Do. & 1400 & 0 & 1,250 & .10 & .04 & $*$ & .44 \\
\hline Do. & 1600 & 0 & 1,370 & .09 & .03 & .042 & .40 \\
\hline Do. & 1800 & 1 & 50 & --- & $*$ & $*$ & $*$ \\
\hline Do. & 2000 & 1 & 170 & .08 & .02 & $*$ & $*$ \\
\hline Do. & 2300 & 1 & 350 & --- & $*$ & $*$ & $*$ \\
\hline $8-14-78$ & 0300 & 1 & 590 & ---- & $*$ & $*$ & $*$ \\
\hline Do. & 0400 & 1 & 650 & ---- & $*$ & $*$ & $*$ \\
\hline Do. & 0600 & 1 & 770 & .07 & .005 & .006 & $*$ \\
\hline
\end{tabular}


Table 71.--A comparison between all tracers used during the third tracer experiment written ir terms of the unitzess concentration ratio $\mathrm{C} / \mathrm{Co}$. The tracers were added simultaneously at 1710, August 12, 1978, and the initial concentrations are given in table 45

[Samples are from an observation well designated 5-m red and screened only for $0.7 \mathrm{~m}$ at $40.0 \mathrm{~m}$ below land surface. This sampler is located at a distance of $5 \mathrm{~m}$ radially from the injection well. The dash (-) indicates sample is available but was not analyzed; an asterisk (*) indicates the sample was not analyzed and is no longer available.]

5-Meter red point well

\begin{tabular}{|c|c|c|c|c|c|c|c|}
\hline \multirow[t]{2}{*}{ Date } & \multirow{2}{*}{$\begin{array}{r}\text { Clock } \\
\text { time }\end{array}$} & \multicolumn{2}{|c|}{ Elapsed } & \multirow[t]{2}{*}{ Boron } & \multirow[t]{2}{*}{ Aniline } & \multirow{2}{*}{$\begin{array}{l}\text { Ethyl- } \\
\text { amine }\end{array}$} & \multirow{2}{*}{$\mathrm{CBr}_{2} \mathrm{~F}_{2}$} \\
\hline & & (d) & $(\mathrm{min})$ & & & & \\
\hline $8-12-78$ & 1710 & 0 & 0 & ---- & $*$ & $*$ & $*$ \\
\hline Do. & 1730 & 0 & 20 & 0.39 & 0.17 & 0.042 & 0.05 \\
\hline Do. & 1800 & 0 & 50 & .54 & .52 & $*$ & .17 \\
\hline Do. & 1817 & 0 & 67 & --- & $*$ & $*$ & .17 \\
\hline Do. & 1830 & 0 & 80 & --- & .63 & .36 & $*$ \\
\hline Do. & 1845 & 0 & 95 & --- & $*$ & $*$ & $*$ \\
\hline Do. & 1900 & 0 & 110 & .66 & .69 & $*$ & .19 \\
\hline Do. & 1930 & 0 & 140 & .69 & .69 & $*$ & .25 \\
\hline Do. & 2000 & 0 & 170 & .75 & .73 & $*$ & .14 \\
\hline Do. & 2030 & 0 & 200 & .79 & .78 & $*$ & $*$ \\
\hline Do. & 2100 & 0 & 230 & .80 & .79 & .57 & .39 \\
\hline Do. & 2130 & 0 & 260 & --- & $*$ & $*$ & * \\
\hline Do. & 2200 & 0 & 290 & .80 & .70 & * & .39 \\
\hline Do. & 2230 & 0 & 320 & --- & .71 & $*$ & .40 \\
\hline Do. & 2300 & 0 & 350 & .85 & .69 & * & .25 \\
\hline Do. & 2330 & 0 & 380 & --- & .69 & $\star$ & .27 \\
\hline Do. & 2400 & 0 & 410 & .65 & .69 & * & .31 \\
\hline $8-13-78$ & 0030 & 0 & 440 & --- & .70 & $*$ & $*$ \\
\hline Do. & 0100 & 0 & 470 & .29 & $*$ & .55 & .24 \\
\hline Do. & 0130 & 0 & 500 & --- & .72 & $*$ & .25 \\
\hline Do. & 0200 & 0 & 530 & .23 & .63 & $*$ & .28 \\
\hline
\end{tabular}


Table 71.--A comparison between all tracers used during the third tracer experiment written in terms of the unitless concentration ratio $\mathrm{C} / \mathrm{Co}$. The tracers were added simultaneously at 1710, August 12, 1978, and the initial concentrations are given in table 45--Continued

\begin{tabular}{|c|c|c|c|c|c|c|c|}
\hline \multicolumn{8}{|c|}{ 5-Meter red point well } \\
\hline \multirow[t]{2}{*}{ Date } & \multirow{2}{*}{$\begin{array}{l}\text { Clock } \\
\text { time }\end{array}$} & \multicolumn{2}{|c|}{$\begin{array}{l}\text { Elapsed } \\
\text { time }\end{array}$} & \multirow[t]{2}{*}{ Boron } & \multirow[t]{2}{*}{ Aniline } & \multirow{2}{*}{$\begin{array}{l}\text { Ethyl- } \\
\text { amine }\end{array}$} & \multirow{2}{*}{$\mathrm{CBr}_{2} \mathrm{~F}_{2}$} \\
\hline & & (d) & $(\min )$ & & & & \\
\hline $8-13-78$ & 0230 & 0 & 560 & --- & 0.34 & 0.25 & 0.26 \\
\hline Do. & 0300 & 0 & 590 & 0.17 & .26 & $*$ & .30 \\
\hline Do. & 0330 & 0 & 620 & --- & $*$ & $*$ & .37 \\
\hline Do. & 0400 & 0 & 650 & .14 & .20 & $\star$ & .21 \\
\hline Do. & 0500 & 0 & 710 & ---- & .14 & $*$ & .21 \\
\hline Do. & 0600 & 0 & 770 & .10 & .10 & .106 & .17 \\
\hline Do. & 0700 & 0 & 830 & --- & .06 & $*$ & .07 \\
\hline Do. & 0800 & 0 & 890 & .07 & .04 & $*$ & .21 \\
\hline Do. & 0900 & 0 & 950 & ---- & $*$ & 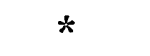 & .16 \\
\hline Do. & 1000 & 0 & 1,010 & ---- & .02 & $*$ & .13 \\
\hline Do. & 1200 & 0 & 1,130 & .06 & .01 & $\star$ & $*$ \\
\hline Do. & 1400 & 0 & 1,250 & --- & .01 & $\dot{*}$ & $*$ \\
\hline Do. & 1600 & 0 & 1,370 & --- & $*$ & $\star$ & $*$ \\
\hline Do. & 1800 & 1 & 50 & ---- & $*$ & $*$ & $\star$ \\
\hline Do. & 2000 & 1 & 170 & .04 & $*$ & $*$ & $*$ \\
\hline Do. & 2300 & 1 & 350 & .04 & $\star$ & $*$ & $*$ \\
\hline
\end{tabular}


Table 72.--A comparison between all tracers used during the third tracer experiment written in terms of the unitzess concentration ratio $\mathrm{C} / \mathrm{Co}$. The tracers were added simultaneously at 1710, August 12, 1978, and the initial concentrations are given in table 45

[Samples are from the point sampler designated 5-m orange located $37.5 \mathrm{~m}$ below 1 and surface and $5 \mathrm{~m}$ radially from the injection well. The dash (-) indicates sample is available but was not analyzed; an asterisk $(*)$ indicates the sample was not analyzed and is no longer available.]

5-Meter orange point sampler

\begin{tabular}{|c|c|c|c|c|c|c|c|}
\hline \multirow[t]{2}{*}{ Date } & \multirow{2}{*}{$\begin{array}{c}\text { Clock } \\
\text { time }\end{array}$} & E] & & \multirow[t]{2}{*}{ Boron } & \multirow[t]{2}{*}{ Aniline } & \multirow{2}{*}{$\begin{array}{l}\text { Ethyl- } \\
\text { amine }\end{array}$} & \multirow{2}{*}{$\mathrm{CBr}_{2} \mathrm{~F}_{2}$} \\
\hline & & (d) & $(\min )$ & & & & \\
\hline $8-12-78$ & 1710 & 0 & 0 & --- & 0 & $*$ & $*$ \\
\hline Do. & 1730 & 0 & 20 & 0.03 & 0 & $*$ & $*$ \\
\hline Do. & 1800 & 0 & 50 & ---- & 0 & $*$ & $*$ \\
\hline Do. & 1830 & 0 & 80 & .03 & 0 & $*$ & $*$ \\
\hline Do. & 1900 & 0 & 110 & --- & .01 & $*$ & $*$ \\
\hline Do. & 1930 & 0 & 140 & .04 & .02 & $*$ & $\star$ \\
\hline Do. & 2000 & 0 & 170 & .06 & .14 & 0 & 0.04 \\
\hline Do. & 2030 & 0 & 200 & ---- & .21 & $\dot{x}$ & $*$ \\
\hline Do. & 2100 & 0 & 230 & --- & .19 & $*$ & .15 \\
\hline Do. & 2130 & 0 & 260 & .08 & .31 & $*$ & .58 \\
\hline Do. & 2200 & 0 & 290 & --- & .34 & $*$ & $\dot{x}$ \\
\hline Do. & 2230 & 0 & 320 & ---- & $*$ & $*$ & .22 \\
\hline Do. & 2300 & 0 & 350 & --- & .47 & 0 & .72 \\
\hline Do. & 2330 & 0 & 380 & ---- & .52 & $*$ & .23 \\
\hline Do. & 2400 & 0 & 410 & .14 & .58 & .042 & .23 \\
\hline $8-13-78$ & 0030 & 0 & 440 & --- & .63 & $*$ & .19 \\
\hline Do. & 0100 & 0 & 470 & .20 & .68 & $*$ & .35 \\
\hline Do. & 0130 & 0 & 500 & ---- & .68 & $*$ & .37 \\
\hline Do. & 0200 & 0 & 530 & ---- & .68 & $*$ & .32 \\
\hline Do. & 0230 & 0 & 560 & .27 & .68 & $*$ & .35 \\
\hline
\end{tabular}


Table 72.--A comparison between all tracers used during the third tracer experiment written in terms of the unitless concentration ratio $\mathrm{C} / \mathrm{Co}$. The tracers were added simultaneously at 1710, August 12, 1978, and the initial concentrations are given in table 45--Continued

\begin{tabular}{|c|c|c|c|c|c|c|c|}
\hline \multicolumn{8}{|c|}{ 5-Meter orange point sampler } \\
\hline \multirow[t]{2}{*}{ Date } & \multirow{2}{*}{$\begin{array}{c}\text { Clock } \\
\text { time }\end{array}$} & \multicolumn{2}{|c|}{$\begin{array}{l}\text { Elapsed } \\
\text { time }\end{array}$} & \multirow[t]{2}{*}{ Boron } & \multirow[t]{2}{*}{ Aniline } & \multirow{2}{*}{$\begin{array}{l}\text { Ethyl- } \\
\text { amine }\end{array}$} & \multirow{2}{*}{$\mathrm{CBr}_{2} \mathrm{~F}_{2}$} \\
\hline & & (d) & (min) & & & & \\
\hline $8-13-78$ & 0300 & 0 & 590 & $-\infty$ & 0.68 & $*$ & 0.31 \\
\hline Do. & 0330 & 0 & 620 & --- & .68 & $*$ & $*$ \\
\hline Do & 0400 & 0 & 650 & --- & .68 & 0.127 & .16 \\
\hline Do. & 0500 & 0 & 710 & 0.31 & .61 & $*$ & .29 \\
\hline Do . & 0600 & 0 & 770 & --- & .52 & 0 & .17 \\
\hline Do. & 0700 & 0 & 830 & .35 & .38 & $*$ & .09 \\
\hline Do. & 0800 & 0 & 890 & --- & .28 & $*$ & .31 \\
\hline Do. & 0900 & 0 & 950 & .33 & .20 & 0 & .37 \\
\hline Do. & 1000 & 0 & 1,010 & --- & .16 & $*$ & .35 \\
\hline Do. & 1200 & 0 & 1,130 & .29 & .12 & $*$ & .33 \\
\hline Do. & 1400 & 0 & 1,250 & .31 & .13 & $*$ & $\star$ \\
\hline Do. & 1600 & 0 & 1,370 & .29 & .12 & 0 & $*$ \\
\hline Do. & 1800 & 1 & 50 & .21 & .06 & $*$ & $*$ \\
\hline Do. & 2000 & 1 & 170 & .19 & .04 & $*$ & $*$ \\
\hline Do. & 2300 & 1 & 350 & .16 & .03 & $*$ & $*$ \\
\hline $8-14-78$ & 0300 & 1 & 590 & .16 & .03 & $*$ & $*$ \\
\hline Do. & 0400 & 1 & 650 & --- & $*$ & $*$ & $*$ \\
\hline Do. & 0600 & 1 & 770 & .12 & $*$ & $*$ & $*$ \\
\hline
\end{tabular}


Table 73.--A comparison between all tracers used during the third tracer experiment written in terms of the unitzess concentration ratio $\mathrm{C} / \mathrm{Co}$. The tracers were added simultaneously at 1710, August 12, 1978, and the initial concentrations are given in table 45

[Samples are from the observation well $10 \mathrm{~m}$ from the injection well and are screened only for a $1.2-\mathrm{m}$ section, $41.5 \mathrm{~m}$ below land surface. The dash (-) indicates sample is available but was not analyzed; an asterisk (*) indicates the sample was not analyzed and is no longer available.]

10-Meter we11

\begin{tabular}{|c|c|c|c|c|c|c|c|}
\hline \multirow[t]{2}{*}{ Date } & \multirow{2}{*}{$\begin{array}{r}\text { Clock } \\
\text { time }\end{array}$} & \multicolumn{2}{|c|}{$\begin{array}{l}\text { Elapsed } \\
\text { time }\end{array}$} & \multirow[t]{2}{*}{ Boron } & \multirow[t]{2}{*}{ Aniline } & \multirow{2}{*}{$\begin{array}{l}\text { Ethy1- } \\
\text { amine }\end{array}$} & \multirow{2}{*}{$\mathrm{CBr}_{2} \mathrm{~F}_{2}$} \\
\hline & & (d) & $(\min )$ & & & & \\
\hline $8-13-78$ & 0100 & 0 & 470 & 0.04 & 0 & $*$ & * \\
\hline Do. & 0300 & 0 & 590 & .04 & .05 & $*$ & $*$ \\
\hline Do. & 0500 & 0 & 710 & .05 & $*$ & $*$ & $*$ \\
\hline Do. & 0600 & 0 & 770 & --- & .04 & $*$ & $*$ \\
\hline Do. & 0700 & 0 & 830 & .08 & .07 & $*$ & $*$ \\
\hline Do. & 0800 & 0 & 890 & --- & .08 & $*$ & $*$ \\
\hline Do. & 0900 & 0 & 950 & .10 & .10 & $*$ & $*$ \\
\hline Do. & 1000 & 0 & 1,010 & --- & .12 & $*$ & $*$ \\
\hline Do. & 1100 & 0 & 1,070 & .12 & .13 & $*$ & $*$ \\
\hline Do. & 1200 & 0 & 1,130 & ---- & .14 & $*$ & $*$ \\
\hline Do. & 1300 & 0 & 1,190 & .14 & .15 & $*$ & $*$ \\
\hline Do. & 1400 & 0 & 1,250 & --- & .15 & $*$ & $*$ \\
\hline Do. & 1500 & 0 & 1,310 & .15 & .15 & $*$ & $*$ \\
\hline Do. & 1600 & 0 & 1,370 & $-\cdots$ & .15 & $*$ & $*$ \\
\hline Do. & 1800 & 1 & 50 & .15 & .14 & $*$ & $*$ \\
\hline Do. & 2000 & 1 & 170 & .15 & $*$ & $*$ & $*$ \\
\hline Do. & 2300 & 1 & 350 & .15 & .12 & * & $\star$ \\
\hline $8-14-78$ & 0300 & 1 & 590 & .14 & .10 & $*$ & $*$ \\
\hline Do. & 0600 & 1 & 770 & .13 & .06 & 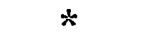 & $*$ \\
\hline Do. & 1100 & 1 & 1,070 & ---- & $*$ & $*$ & $*$ \\
\hline Do. & 1300 & 1 & 1,190 & .11 & .04 & $*$ & $*$ \\
\hline
\end{tabular}


Table 74.--A comparison between all tracers used during the third tracer experiment written in terms of the unitless concentration ratio $\mathrm{C} / \mathrm{Co}$. The tracers were added simultaneously at 1710, August 12, 1978, and the initial concentrations are given in table 45

[Samples are from the observation well $15 \mathrm{~m}$ from the injection well which is screened for the saturated thickness of the aquifer. The dash (-) indicates sample is available but was not analyzed; an asterisk (*) indicates the sample was not analyzed and is no longer available.]

15-Meter we11

\begin{tabular}{|c|c|c|c|c|c|c|c|}
\hline \multirow[t]{2}{*}{ Date } & \multirow{2}{*}{$\begin{array}{r}\text { Clock } \\
\text { time }\end{array}$} & \multicolumn{2}{|c|}{ Elapsed } & \multirow[t]{2}{*}{ Boron } & \multirow[t]{2}{*}{ Aniline } & \multirow{2}{*}{$\begin{array}{l}\text { Ethyl- } \\
\text { amine }\end{array}$} & \multirow{2}{*}{$\mathrm{CBr}_{2} \mathrm{~F}_{2}$} \\
\hline & & (d) & (min) & & & & \\
\hline $8-13-78$ & 0100 & 0 & 470 & 0.04 & $*$ & $\star$ & $*$ \\
\hline Do. & 0300 & 0 & 590 & .04 & $*$ & $*$ & $*$ \\
\hline Do. & 0500 & 0 & 710 & .05 & 0.005 & $*$ & $*$ \\
\hline Do. & 0600 & 0 & 770 & ---- & .02 & $*$ & $*$ \\
\hline Do. & 0700 & 0 & 830 & .06 & .02 & $*$ & $\star$ \\
\hline Do. & 0800 & 0 & 890 & ---- & .03 & $*$ & $\star$ \\
\hline Do. & 0900 & 0 & 950 & .07 & .04 & $*$ & 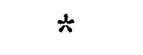 \\
\hline Do. & 1000 & 0 & 1,010 & ---- & $*$ & $*$ & $*$ \\
\hline Do. & 1100 & 0 & 1,070 & .07 & .04 & $*$ & $*$ \\
\hline Do. & 1200 & 0 & 1,130 & ---- & .04 & $*$ & $*$ \\
\hline Do. & 1300 & 0 & 1,190 & .08 & .04 & $*$ & $\star$ \\
\hline Do. & 1400 & 0 & 1,250 & --- & .05 & $\star$ & $*$ \\
\hline Do. & 1500 & 0 & 1,310 & .08 & .05 & $*$ & $*$ \\
\hline Do. & 1600 & 0 & 1,370 & --- & .05 & $*$ & $\star$ \\
\hline Do. & 1800 & 1 & 50 & .08 & $*$ & $*$ & $*$ \\
\hline Do. & 2000 & 1 & 170 & .08 & .05 & $*$ & $*$ \\
\hline Do. & 2300 & 1 & 350 & .09 & .05 & $*$ & $\star$ \\
\hline $8-14-78$ & 0300 & 1 & 590 & .08 & .05 & $*$ & $*$ \\
\hline Do. & 0600 & 1 & 770 & .09 & .05 & $*$ & $*$ \\
\hline Do. & 1100 & 1 & 1,070 & --- & $*$ & $*$ & $*$ \\
\hline Do. & 1300 & 1 & 1,190 & .09 & .03 & $*$ & $*$ \\
\hline
\end{tabular}




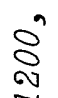

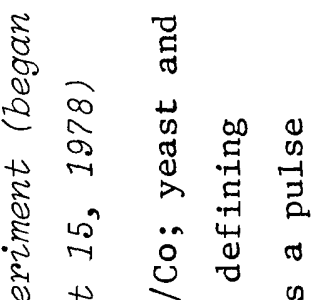

茛

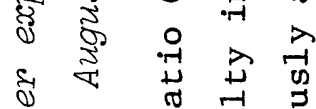

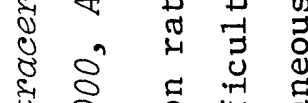

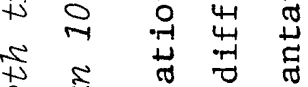

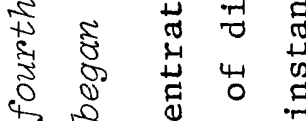

के

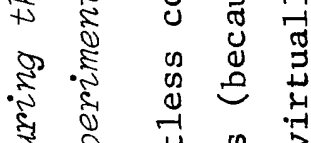

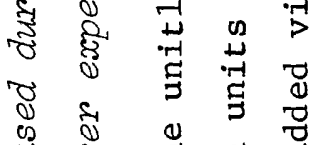

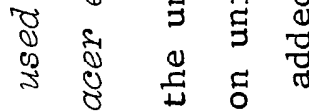

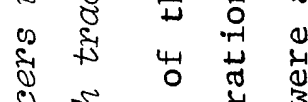

ठำ

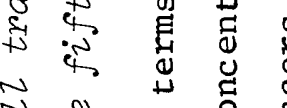

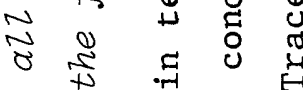

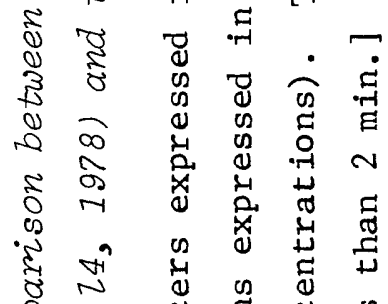

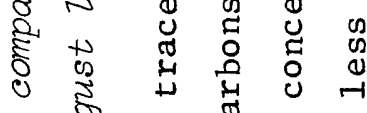

广

! ख

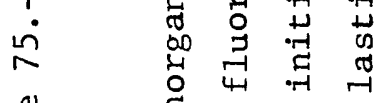

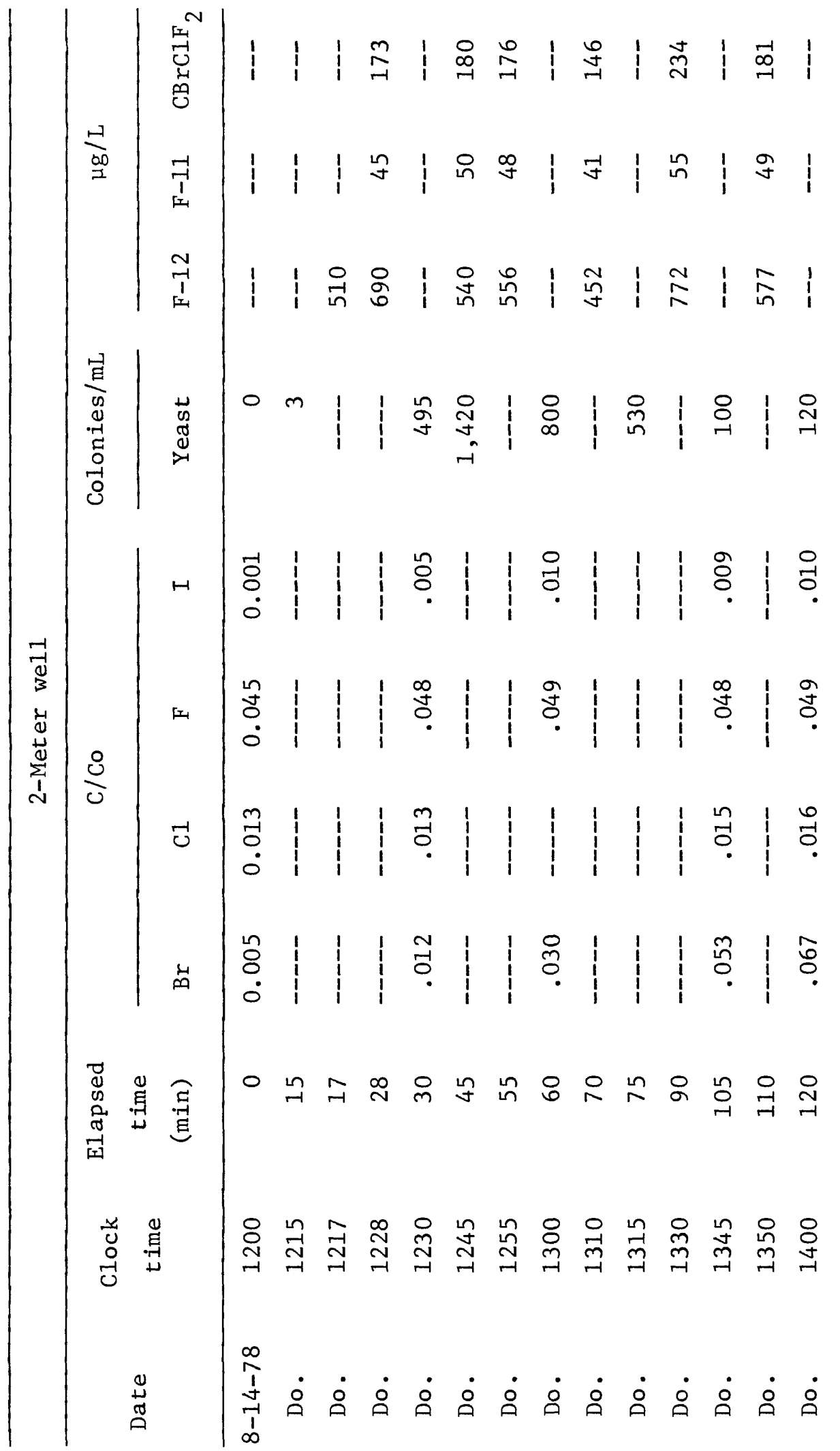




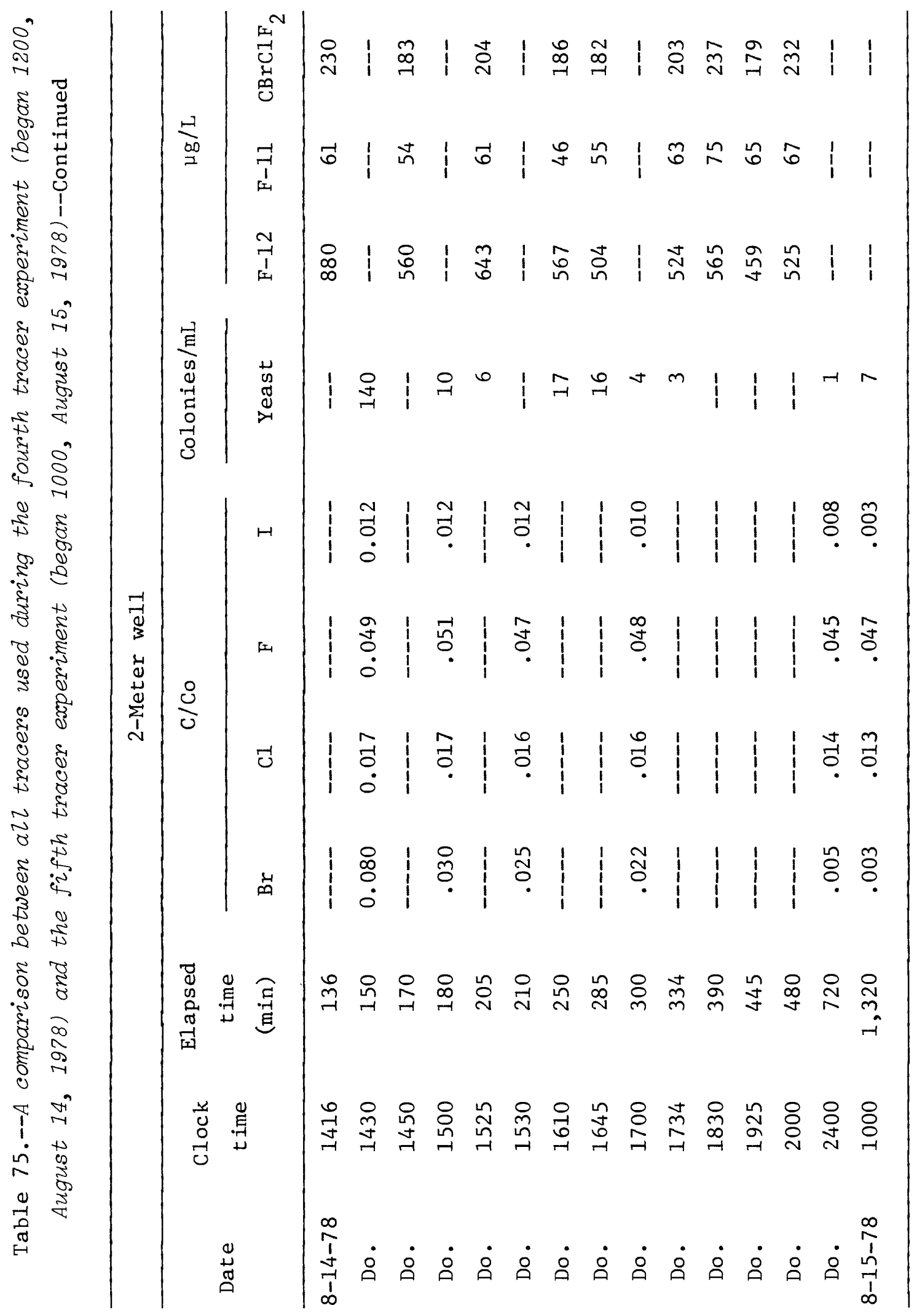




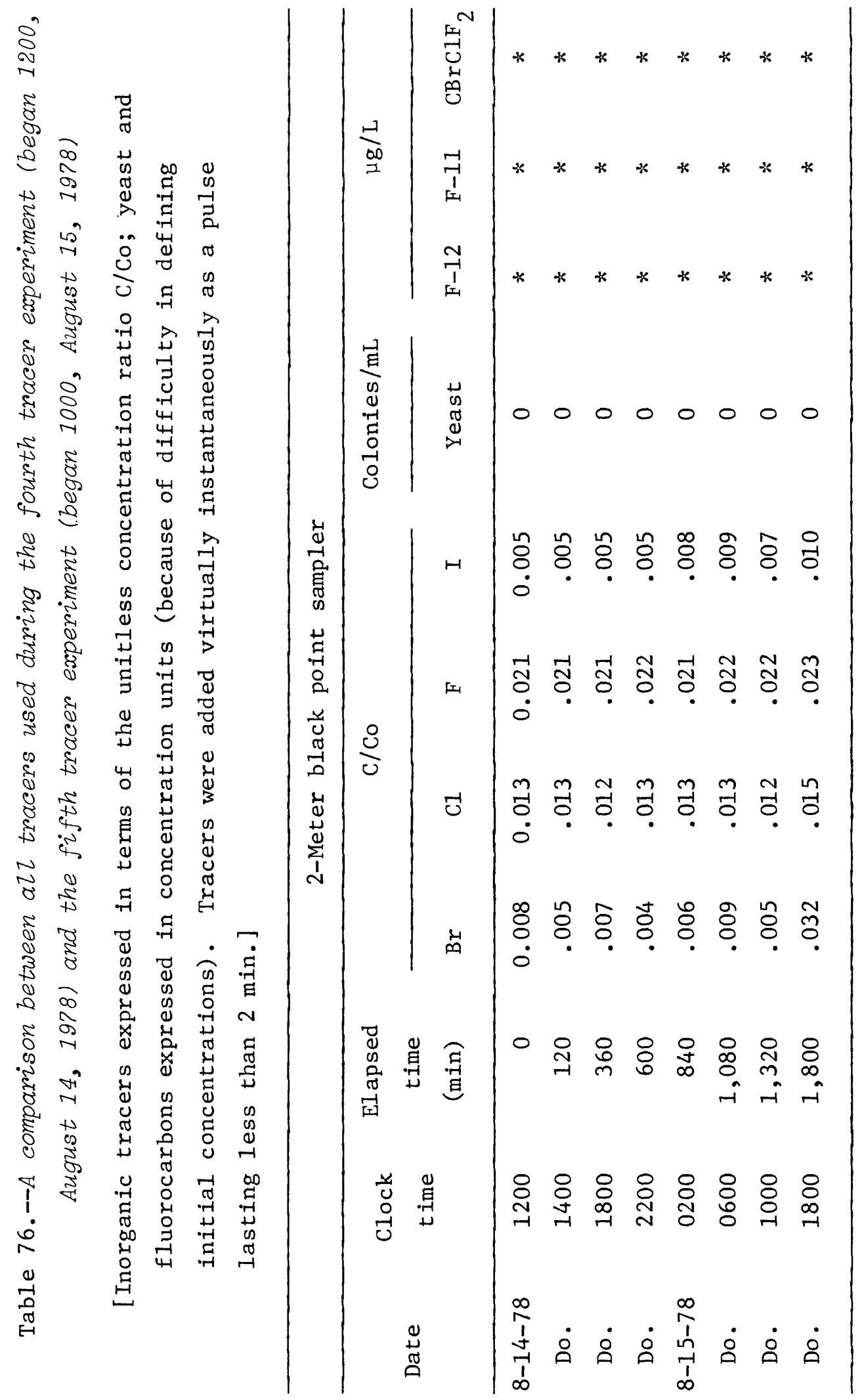




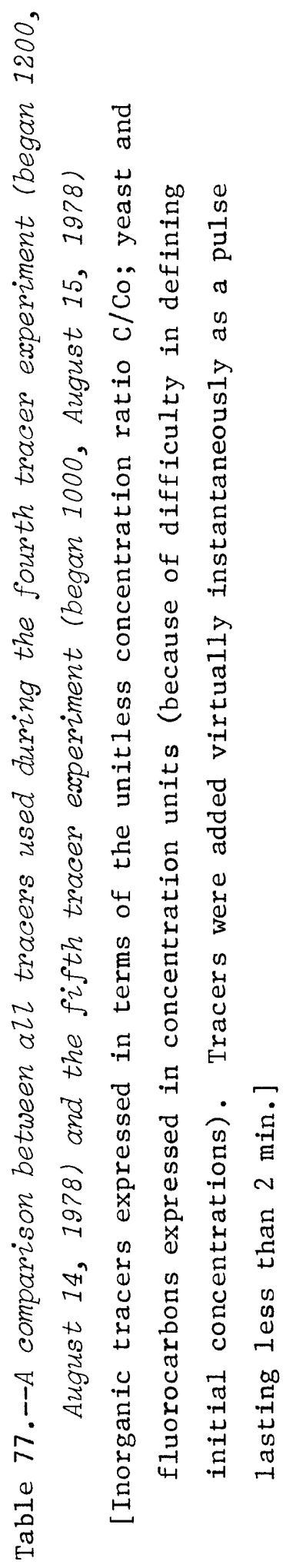

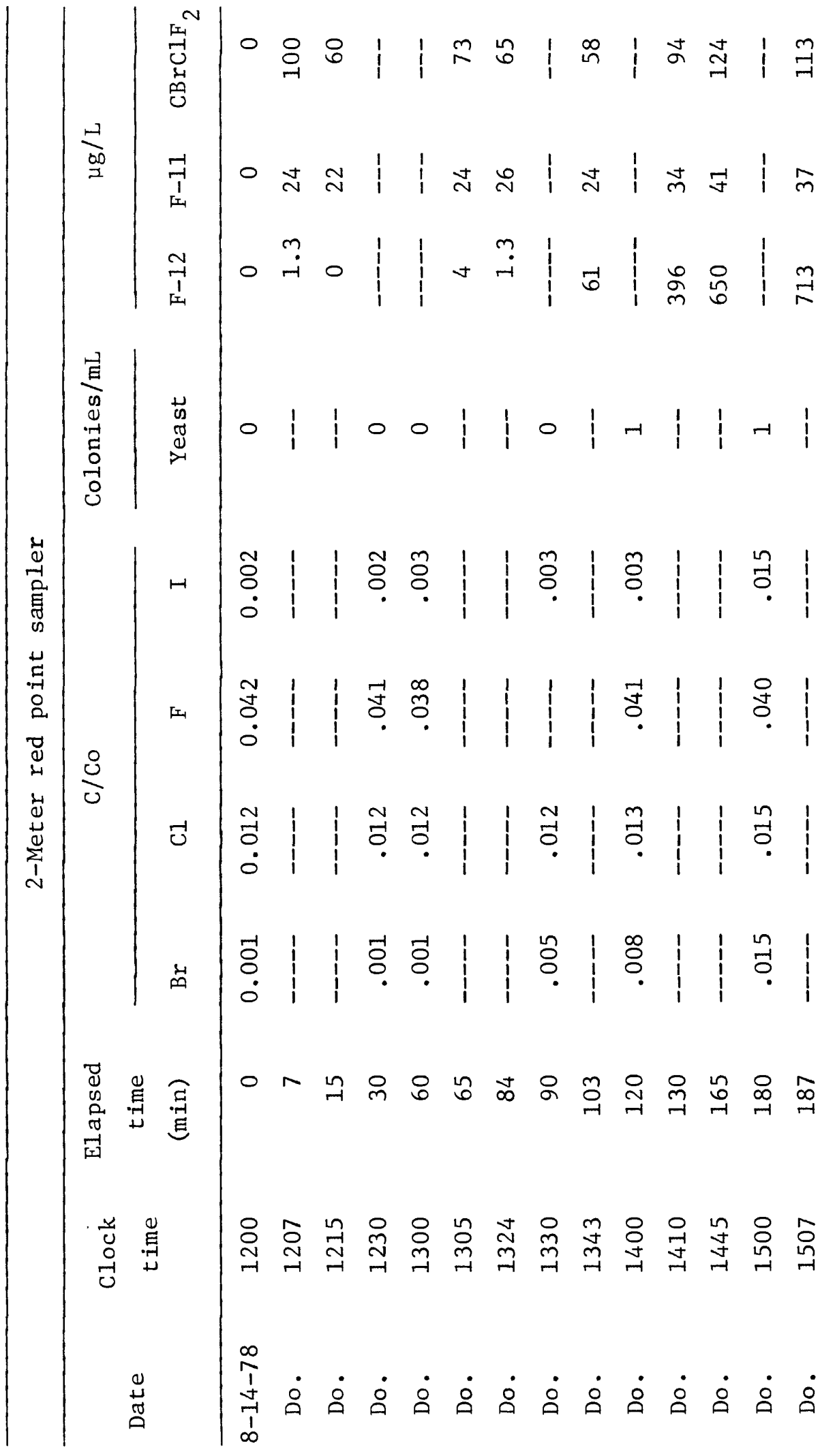




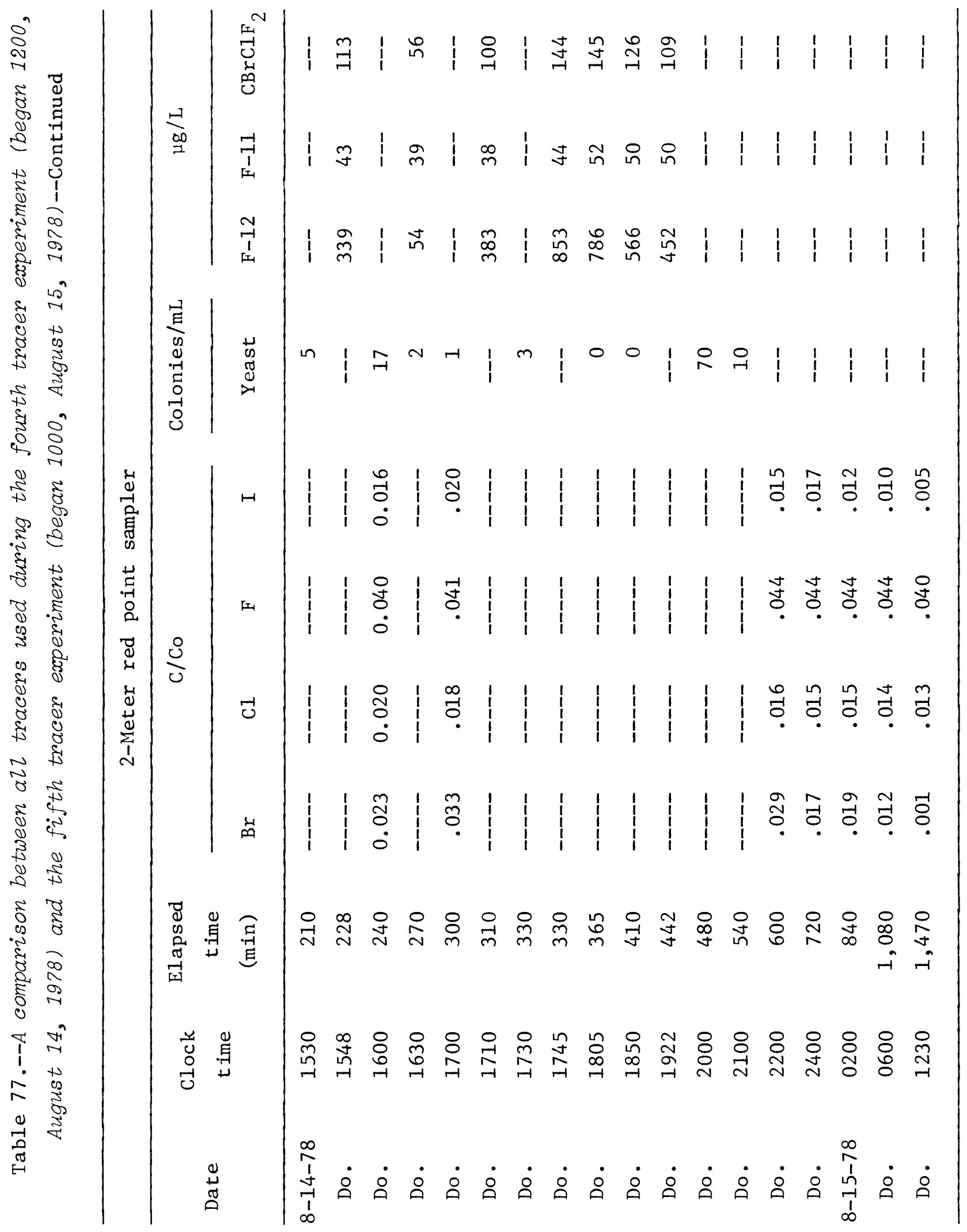




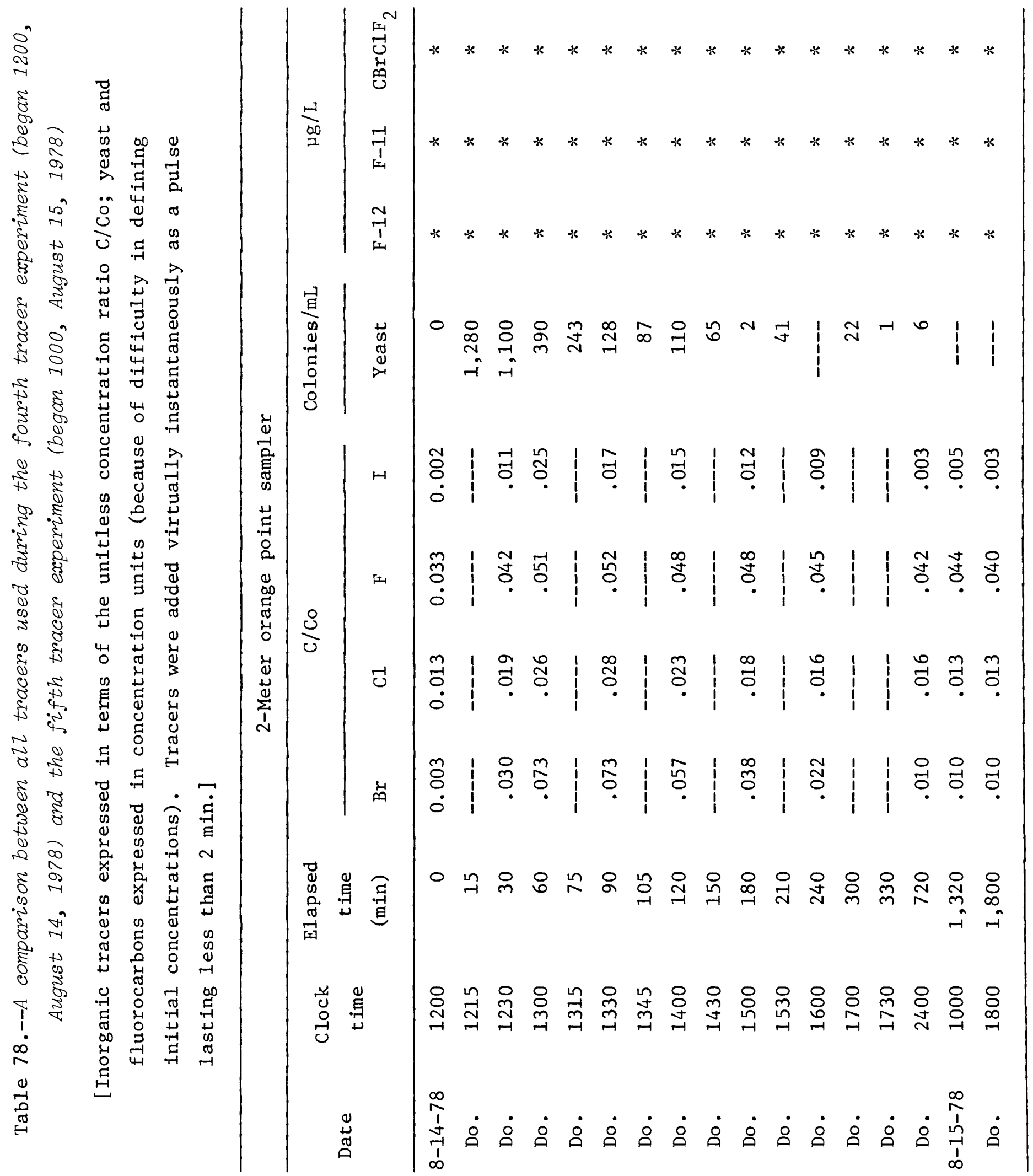




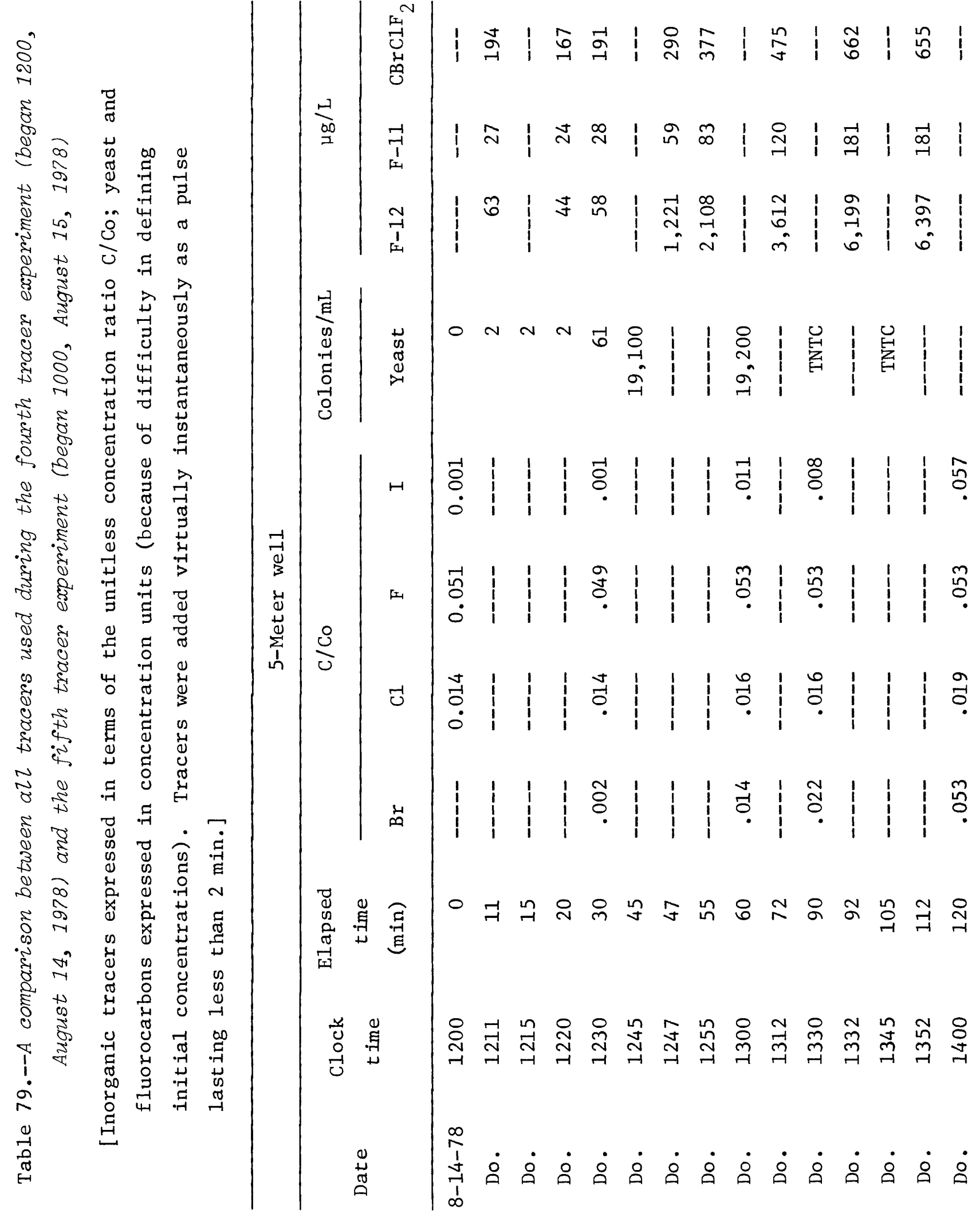




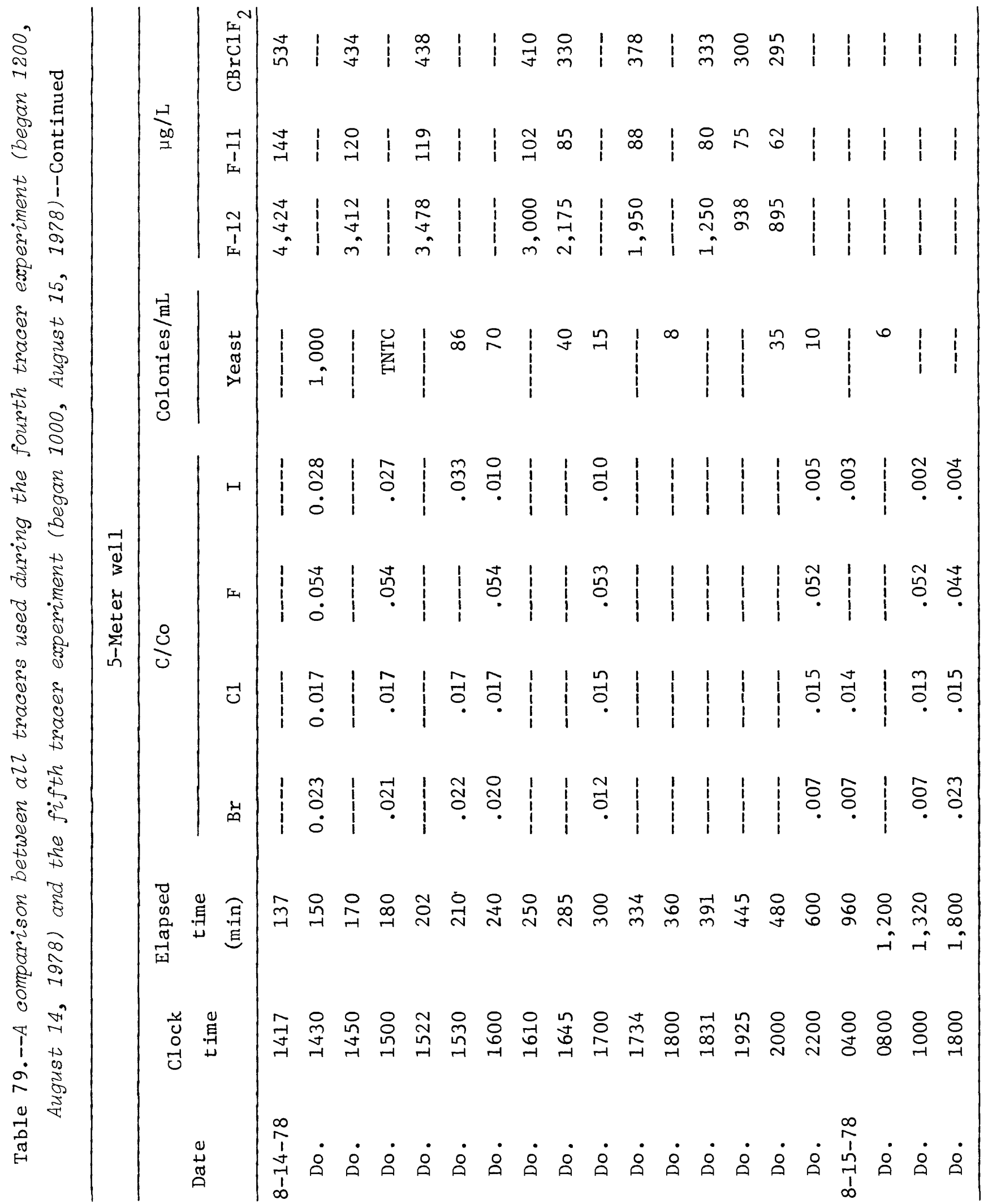




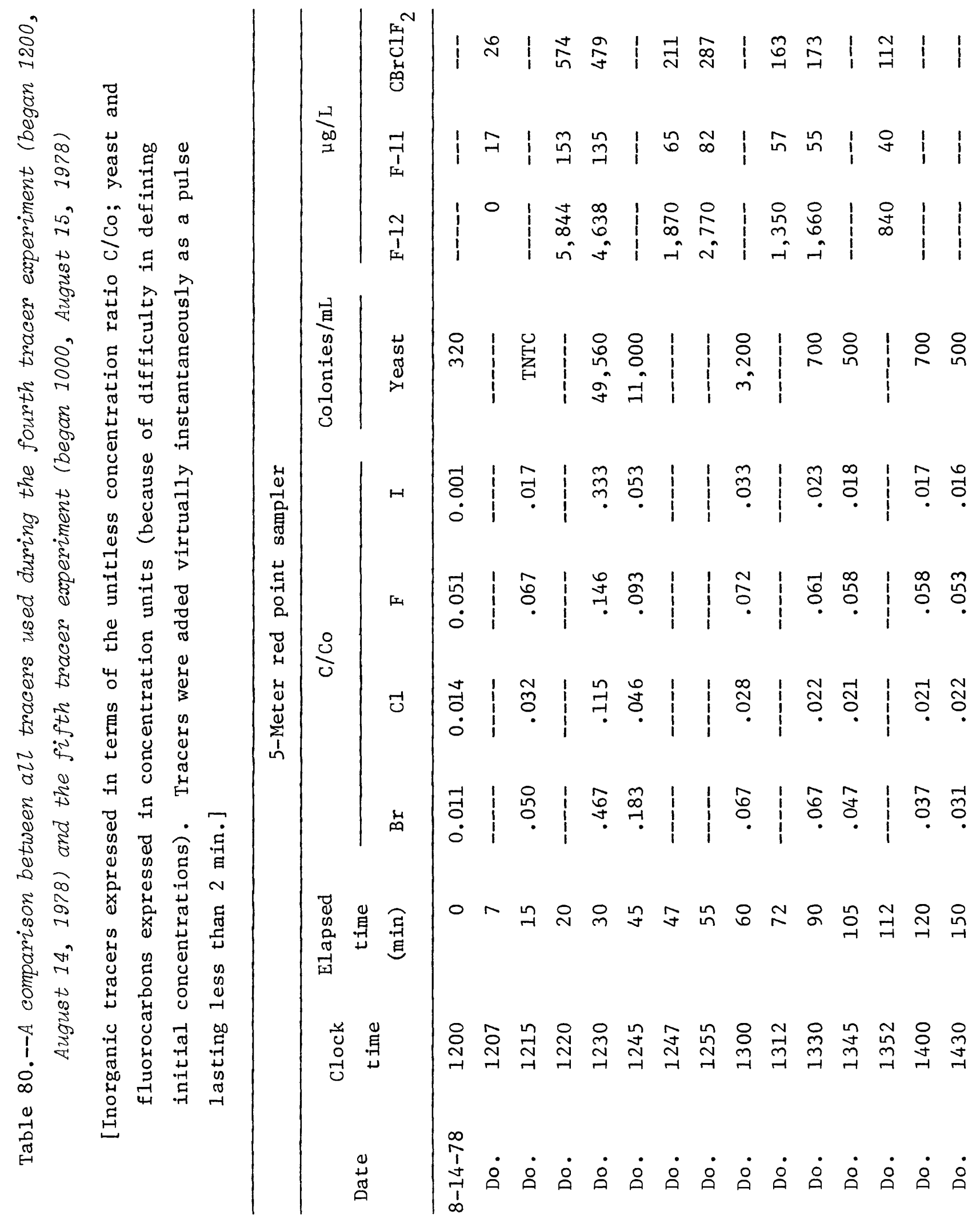




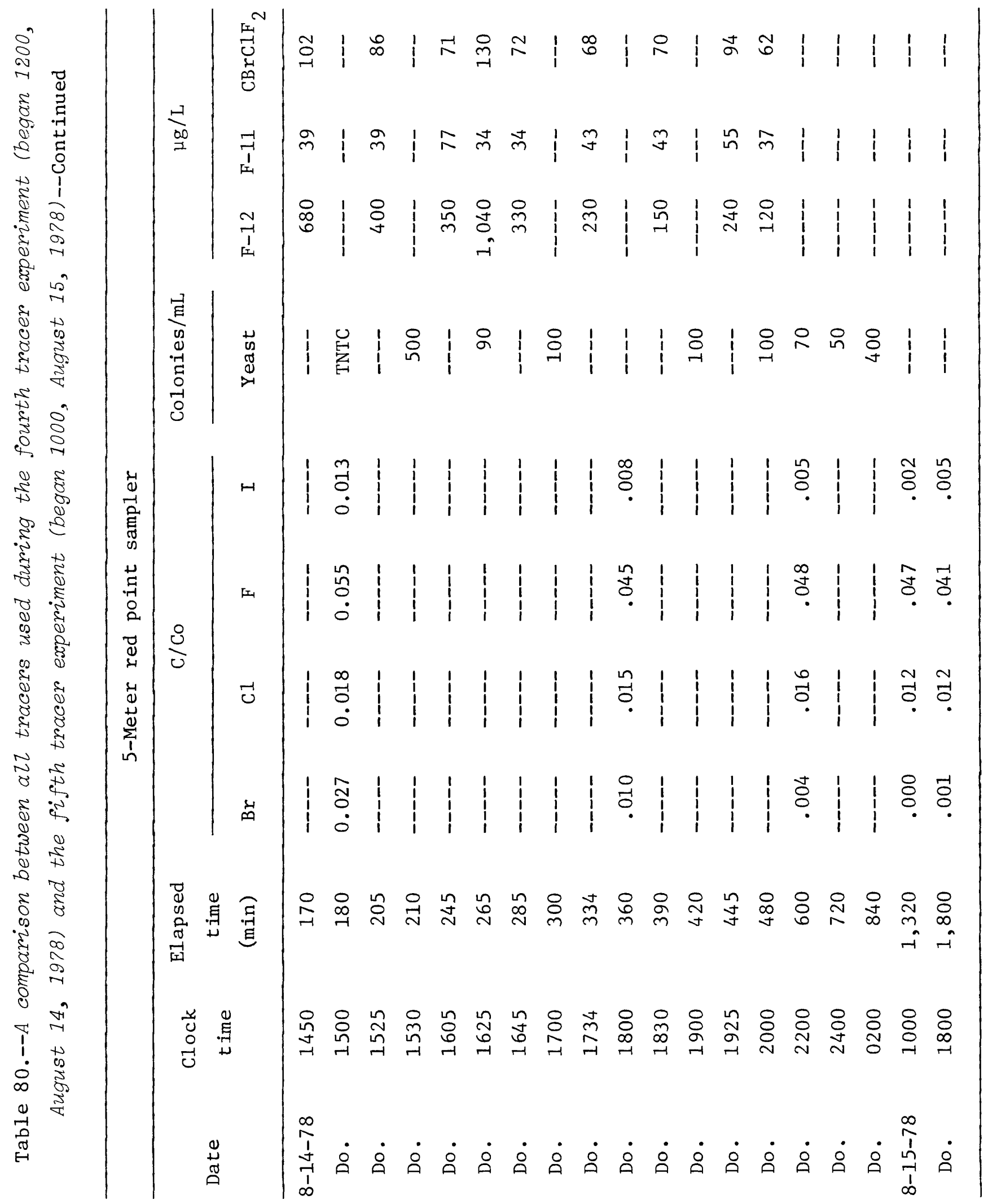



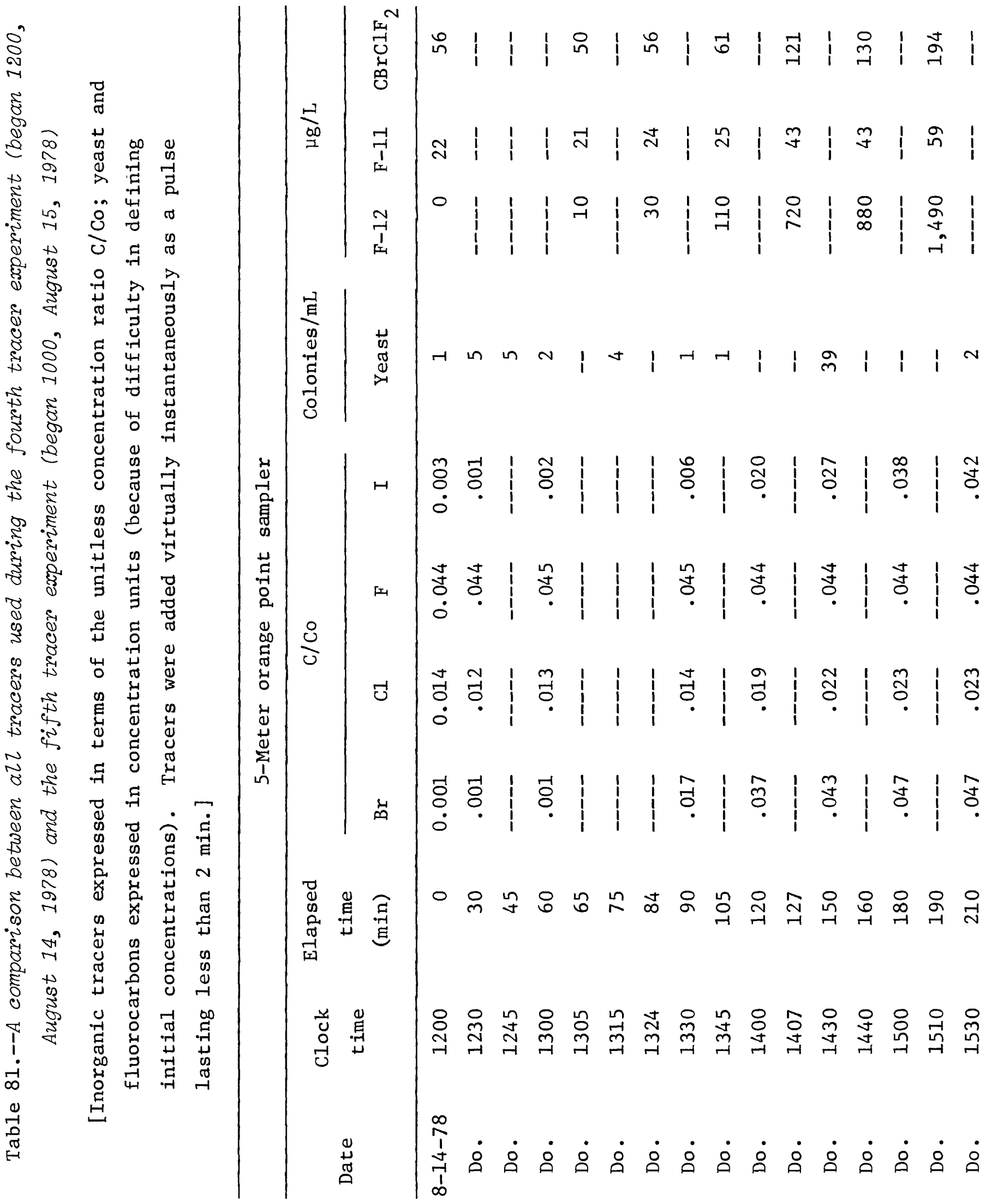


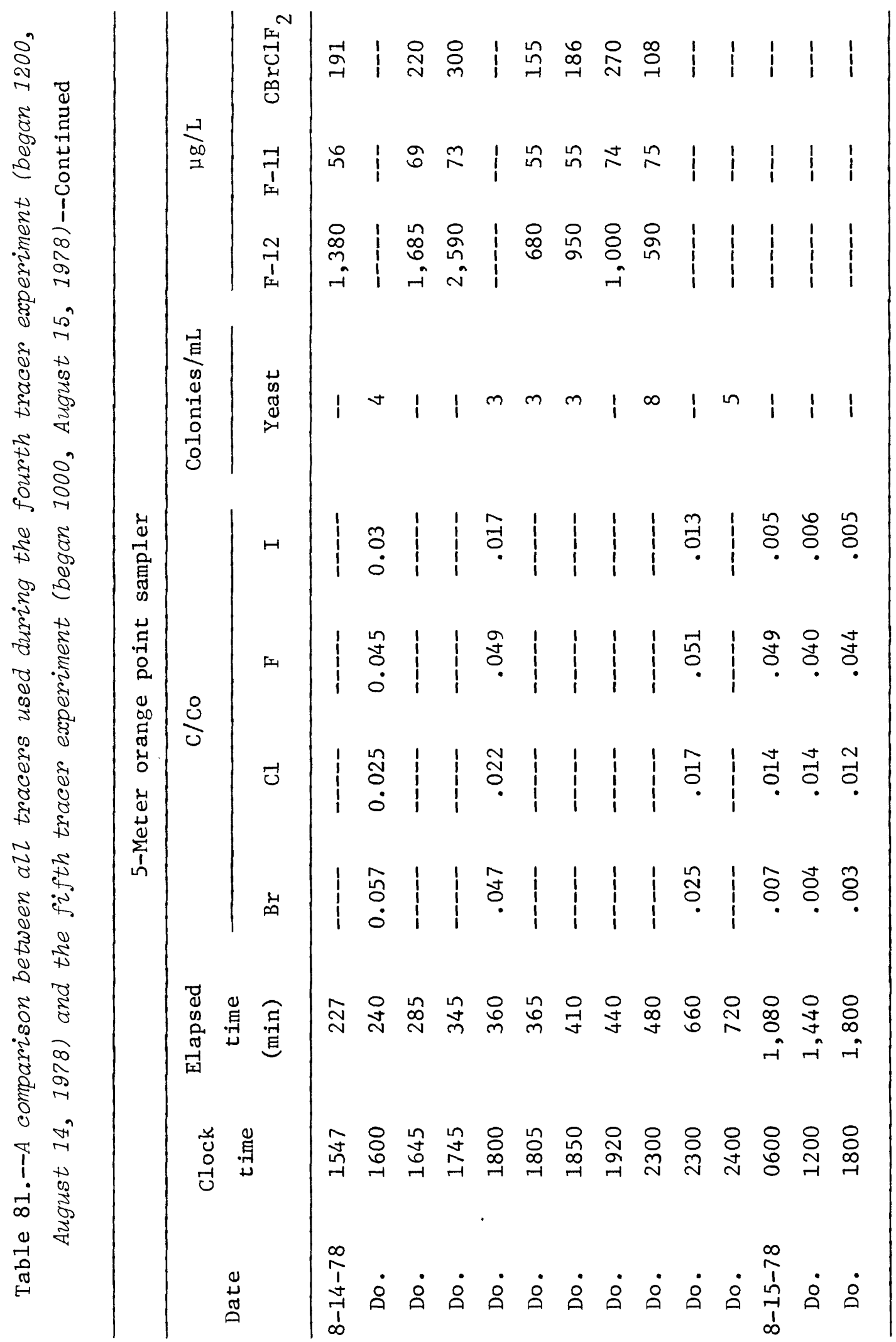




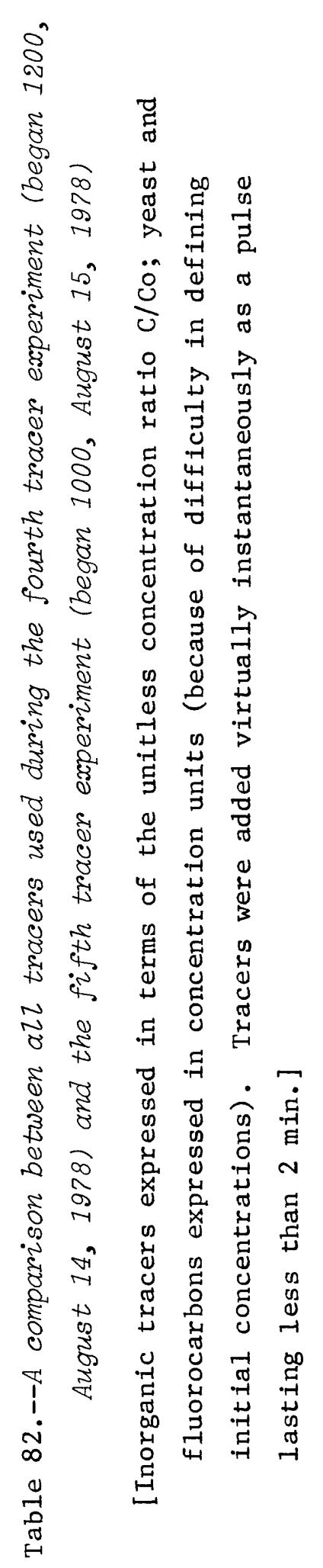

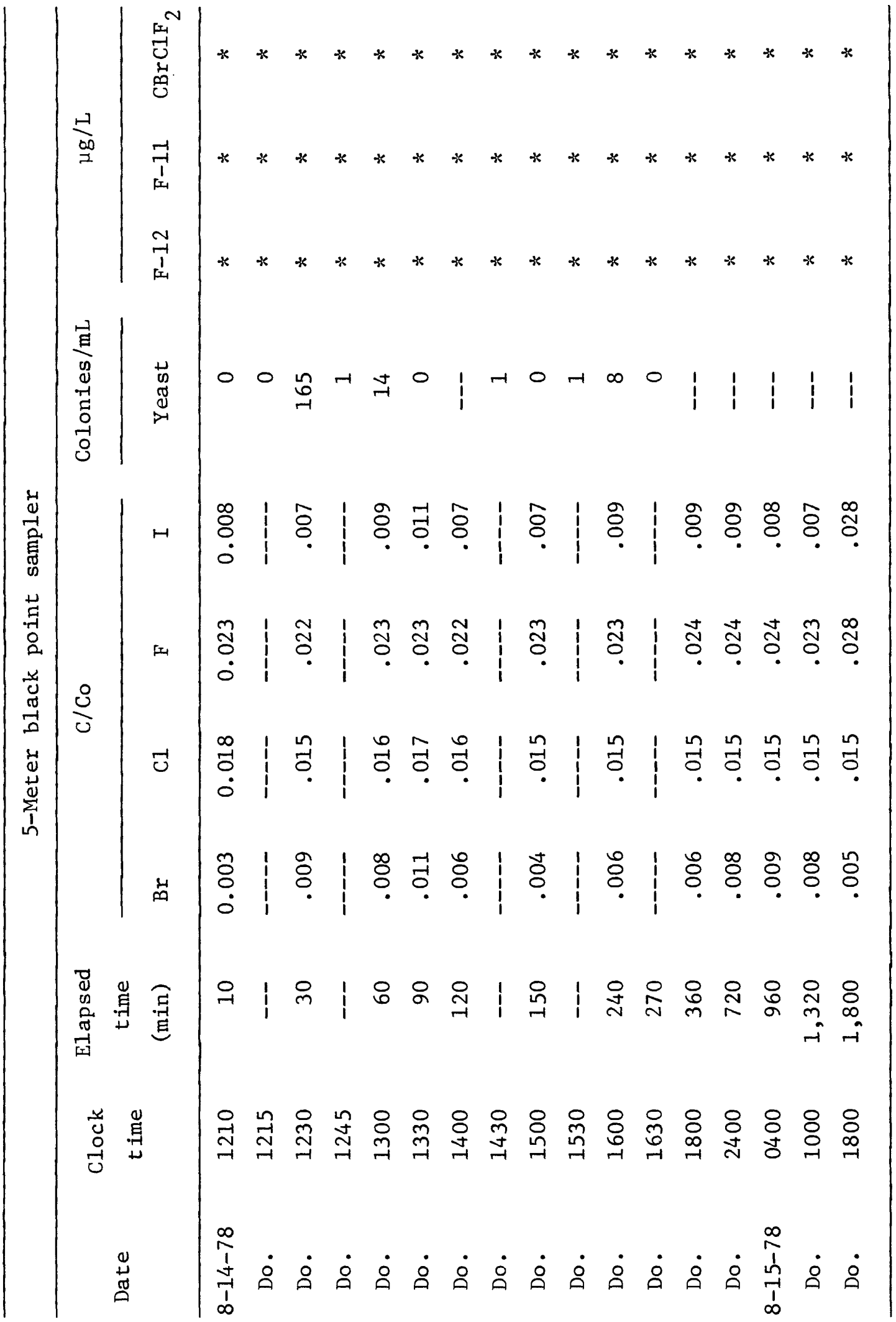




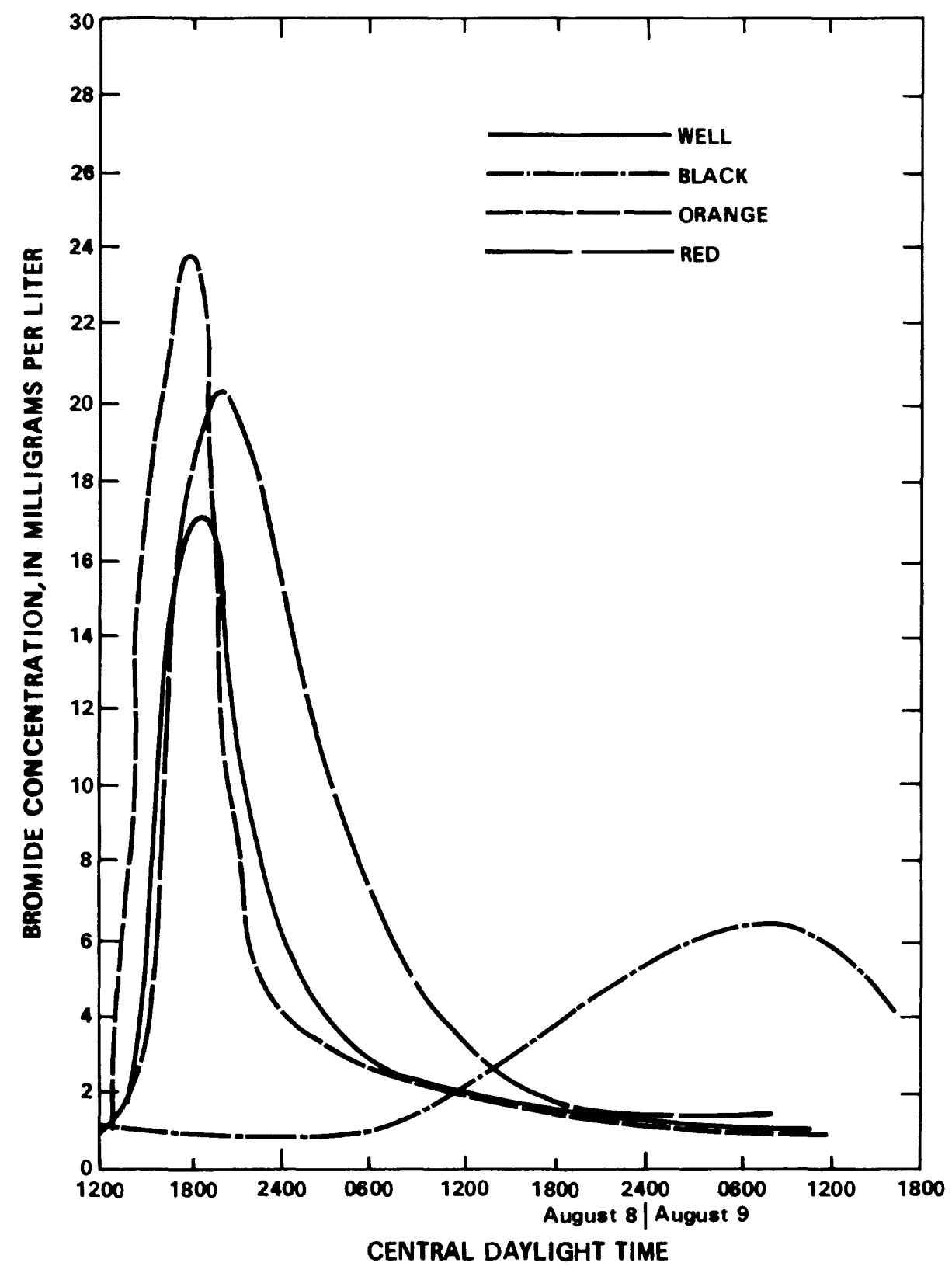

Figure 19.--Bromide data obtained from the 2-meter well and point samplers during the first experiment of the August 1978 test.

(Data are from tables 47-50.) 


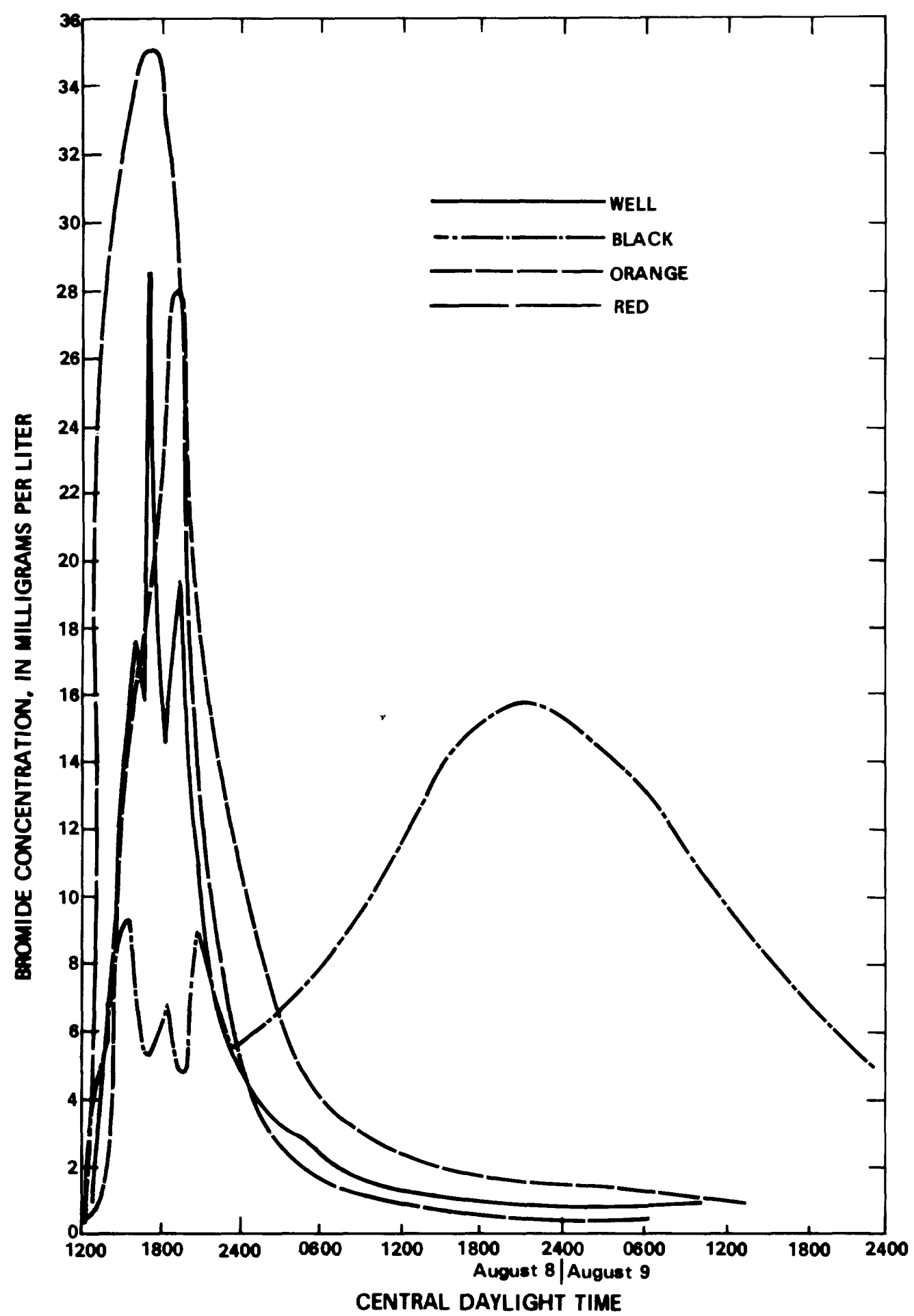

Figure 20.--Bromide data obtained from the 5-meter well and point samplers during the first experiment of the August 1978 test.

(Data are from tables 51-54.) 


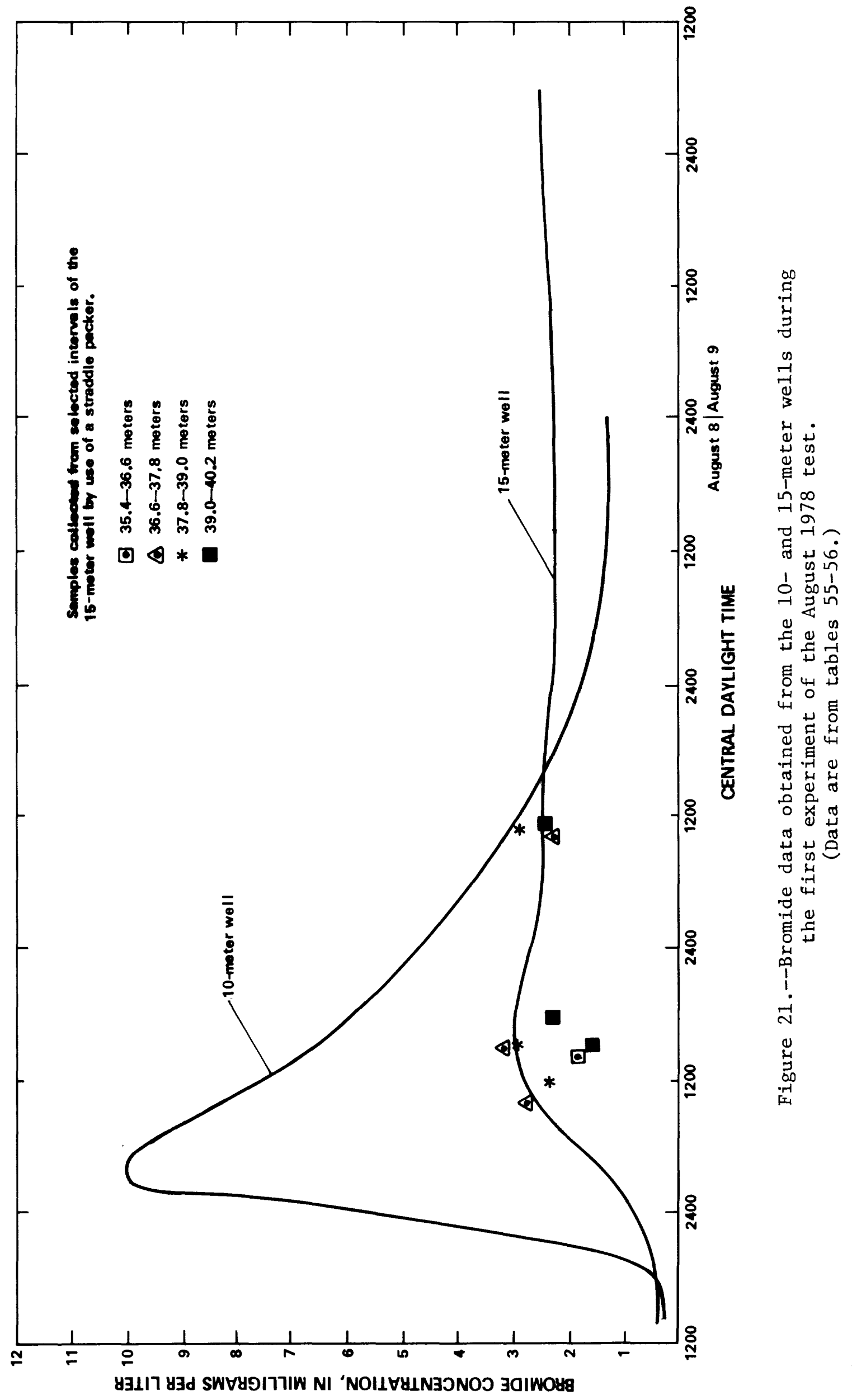




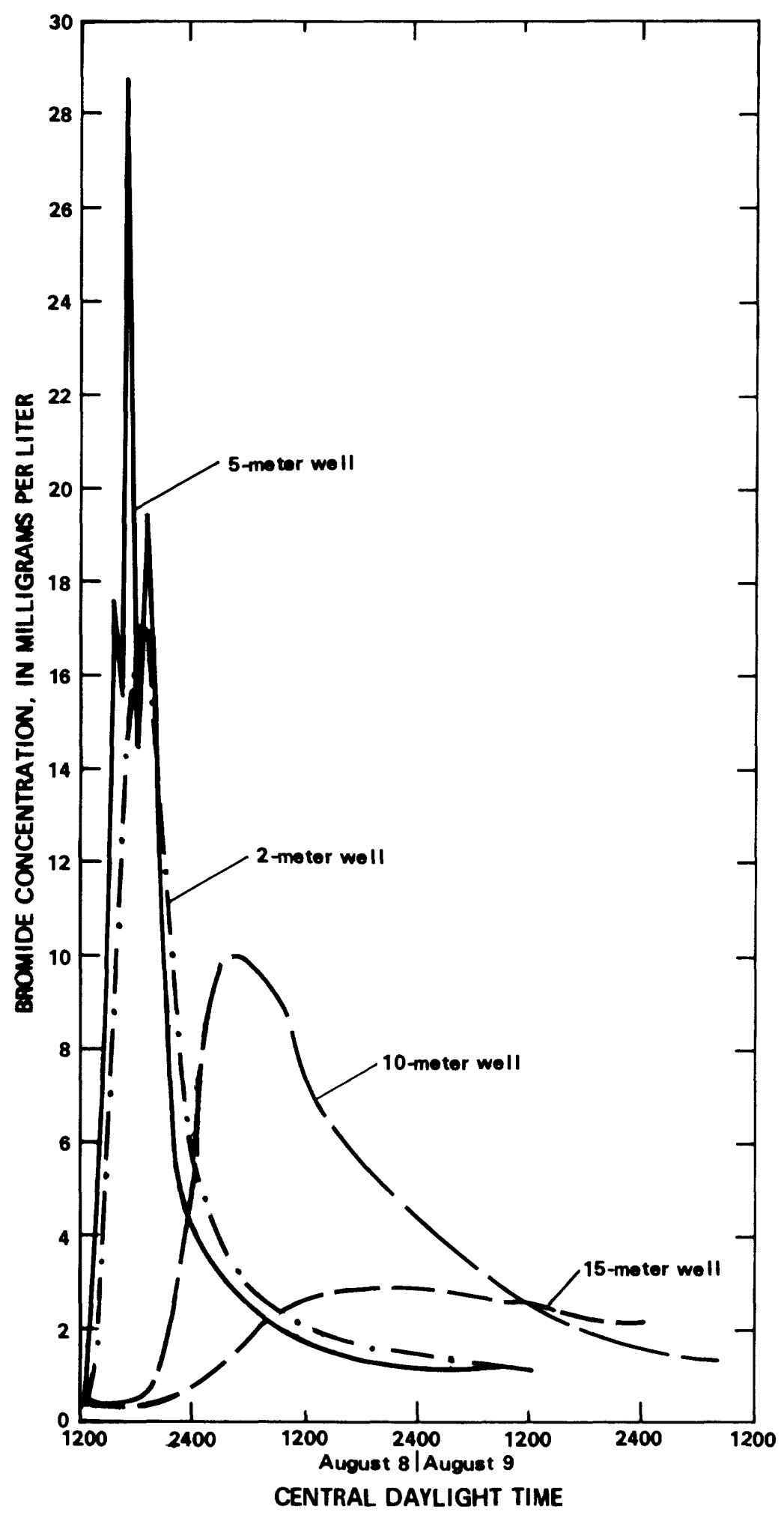

Figure 22.--Bromide data obtained from the 2-, 5-, 10-, and 15-meter wells during the first experiment of the August 1978 test. (Data are from tables 47-56.) 


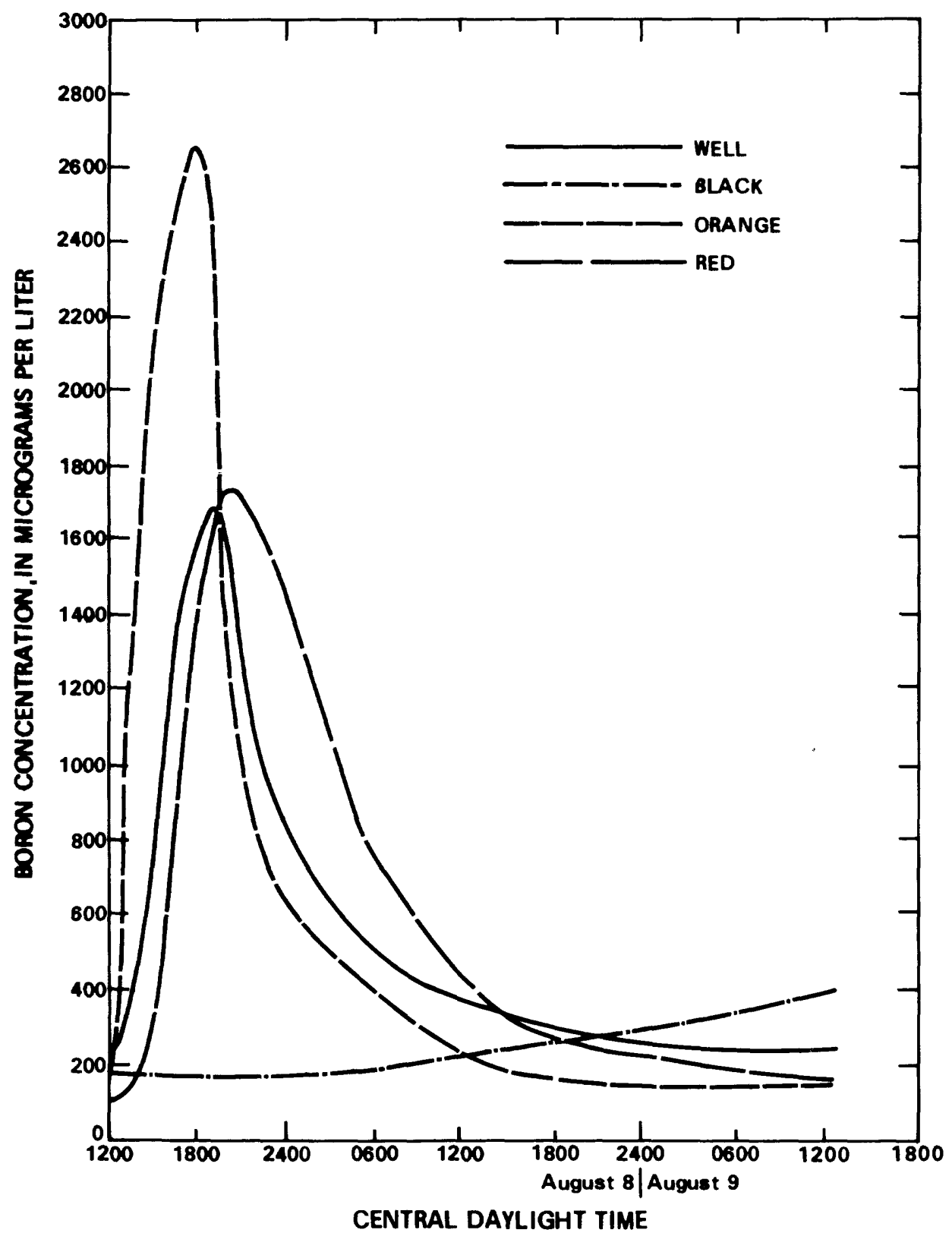

Figure 23.--Boron data obtained from the 2-meter well and point samplers during the first experiment of the August 1978 test.

(Data are from tables 47-50.) 


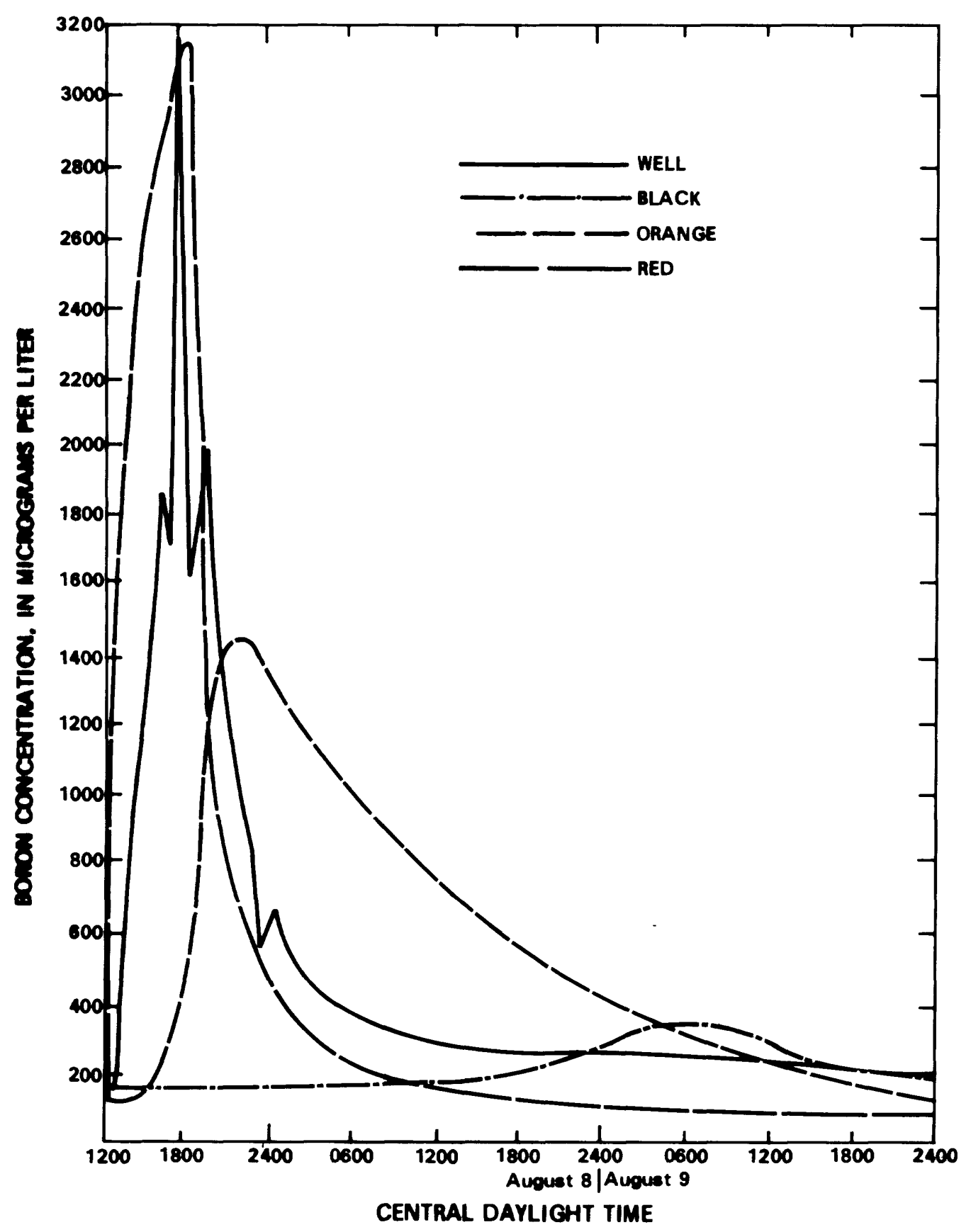

Figure 24.--Boron data obtained from the 5-meter well and point samplers during the first experiment of the August 1978 test. (Data are from tables 51-54.) 


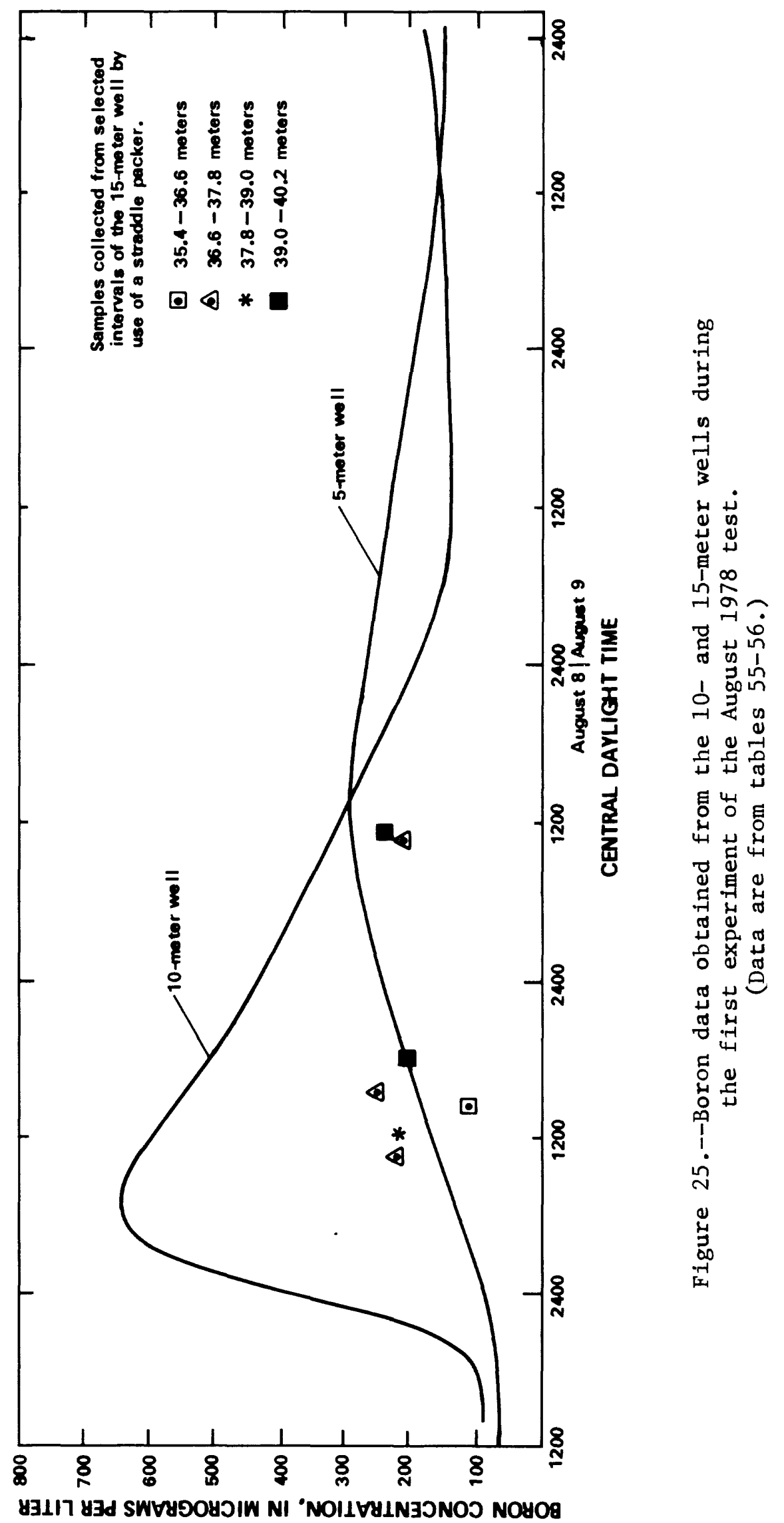




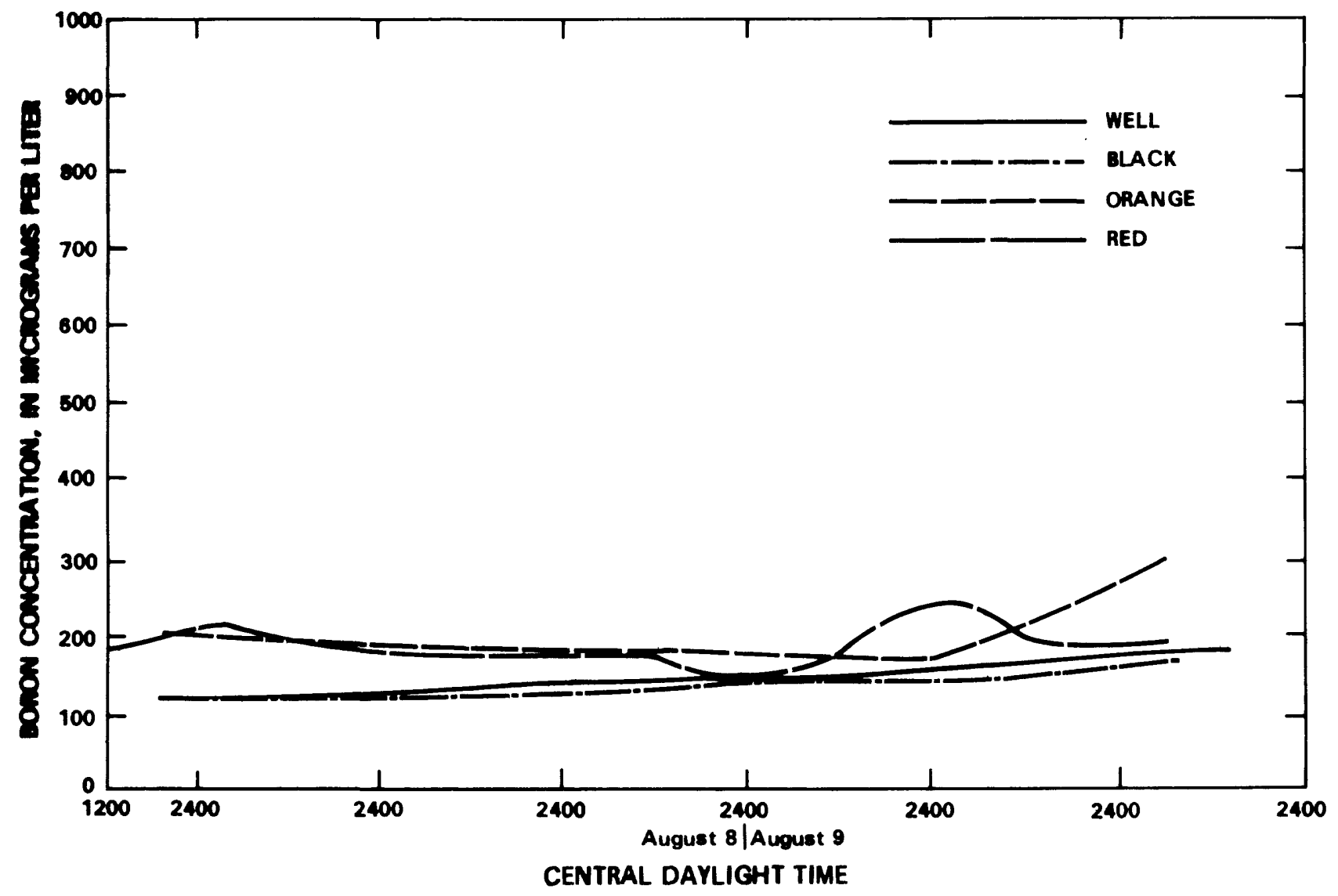

Figure 26.--Boron data obtained from the 30-meter well and point samplers during the first experiment of the August 1978 test. (Data are from tables 57-60.) 


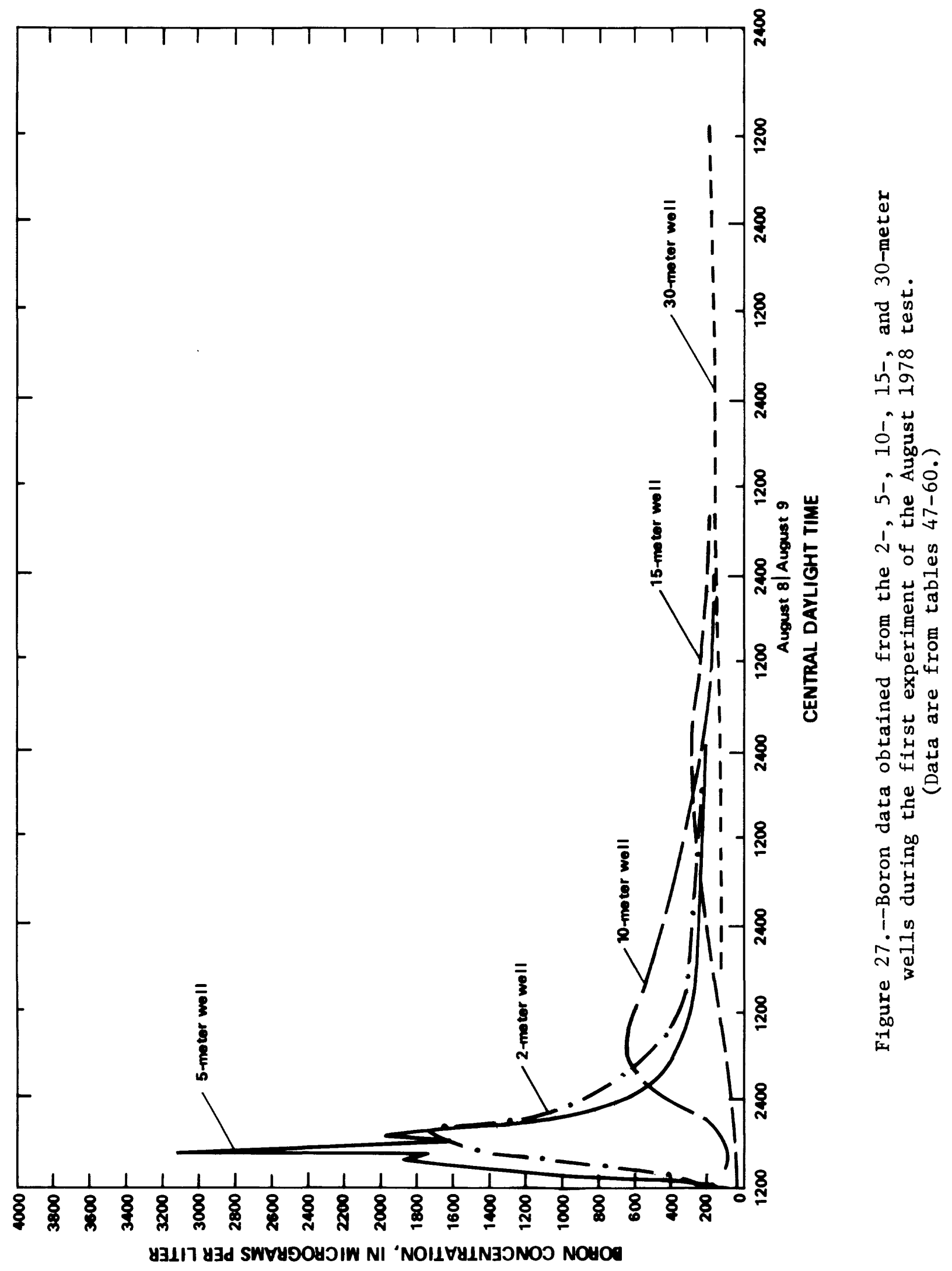




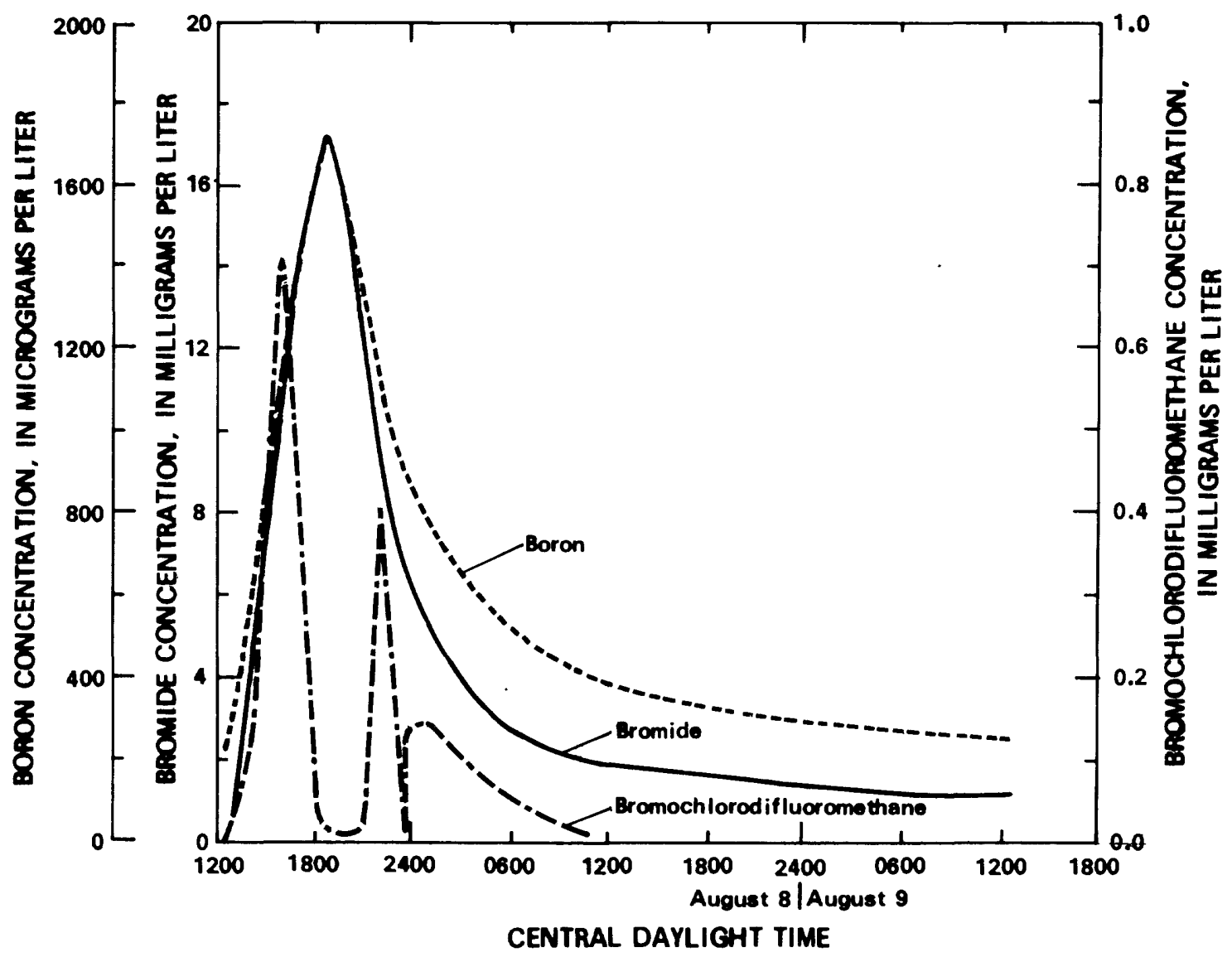

Figure 28.--Comparison of boron, bromide, and bromochlorodifluoromethane data obtained from the 2-meter well during the first experiment of the August 1978 test.

(Data are from table 47.) 


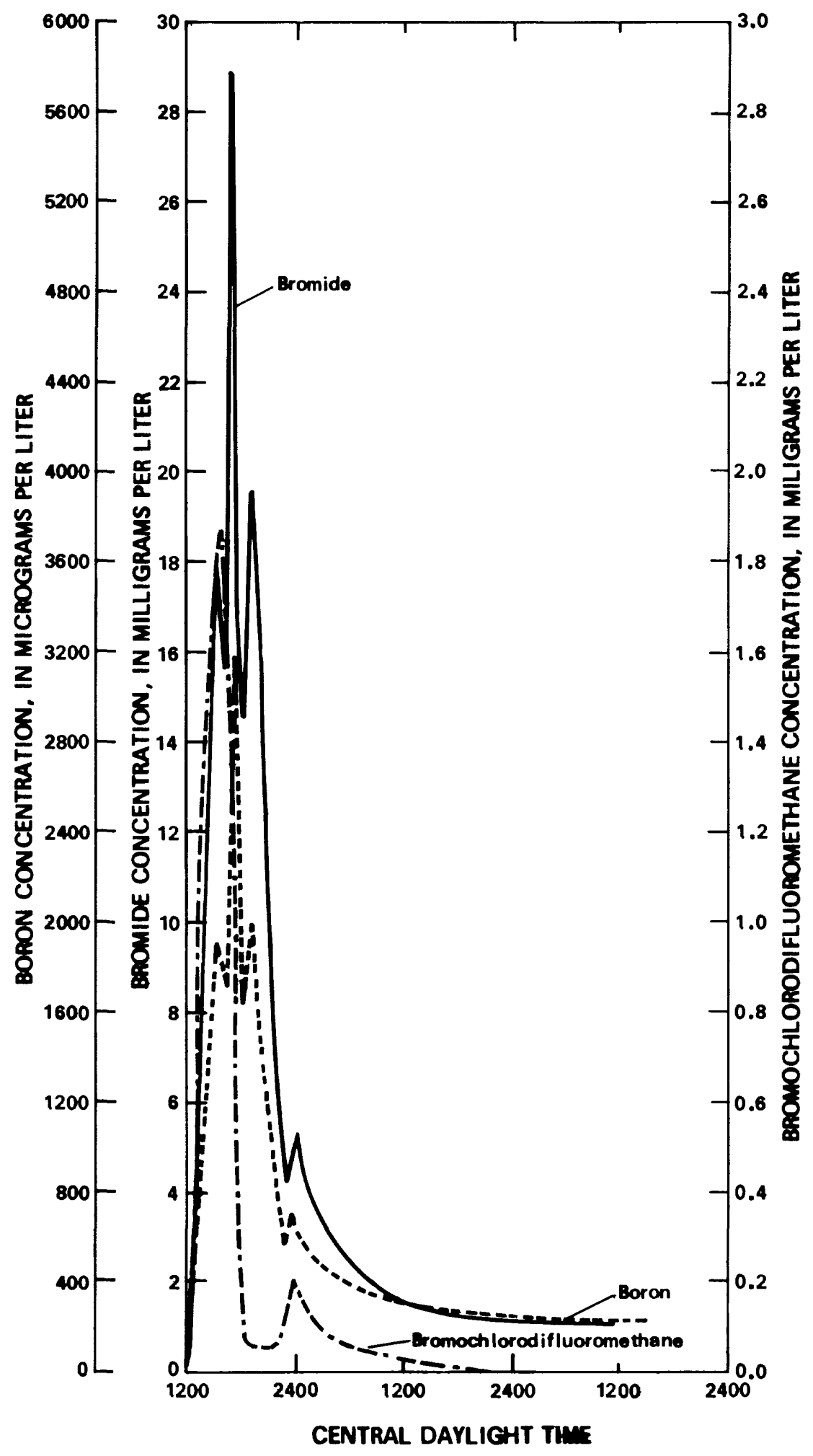

Figure 29.--Comparison of boron, bromide, and bromochlorodifluoromethane data obtalned from the 5-meter well during the first

experiment of the August 1978 test.

(Data are from table 51.) 


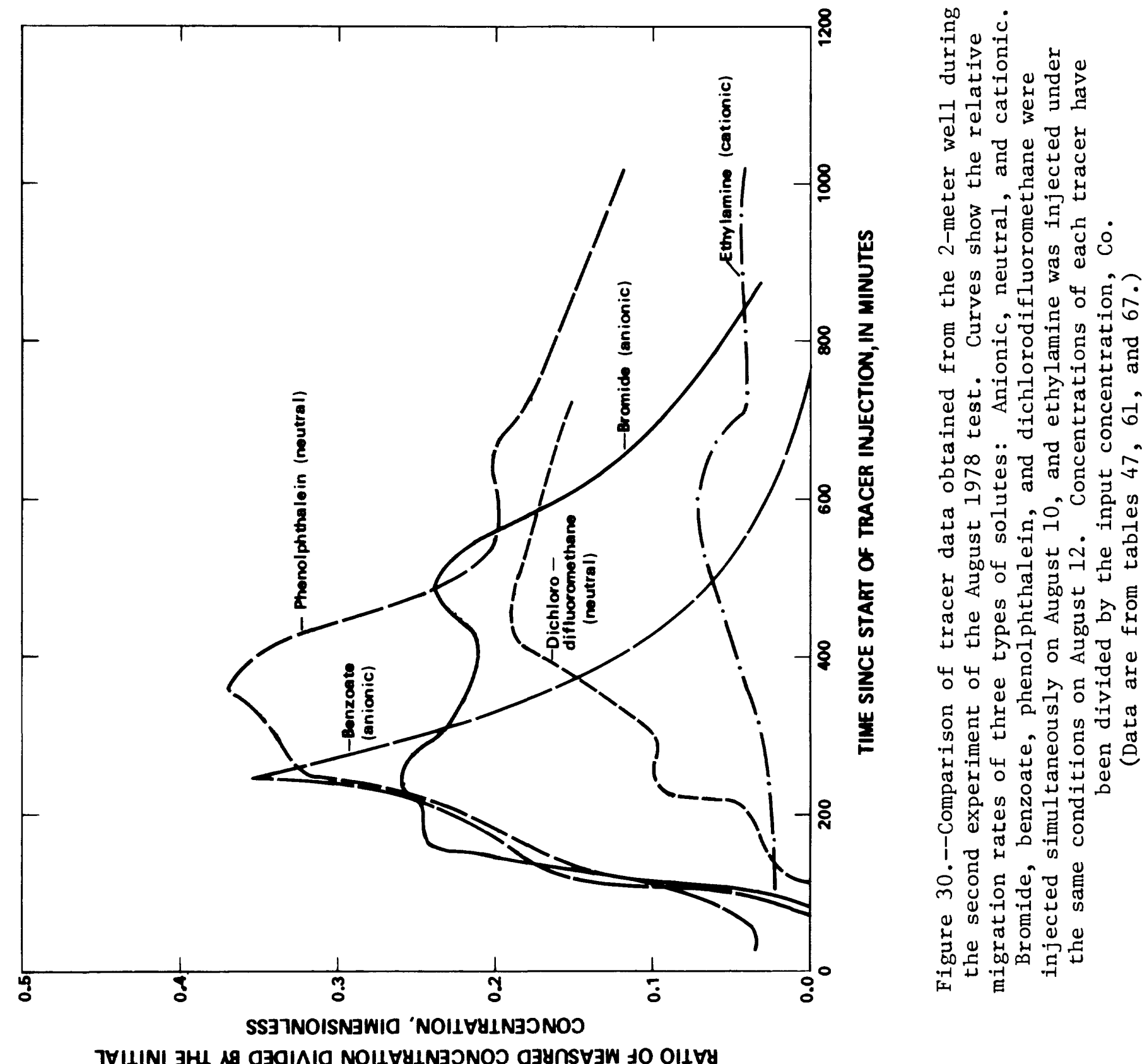




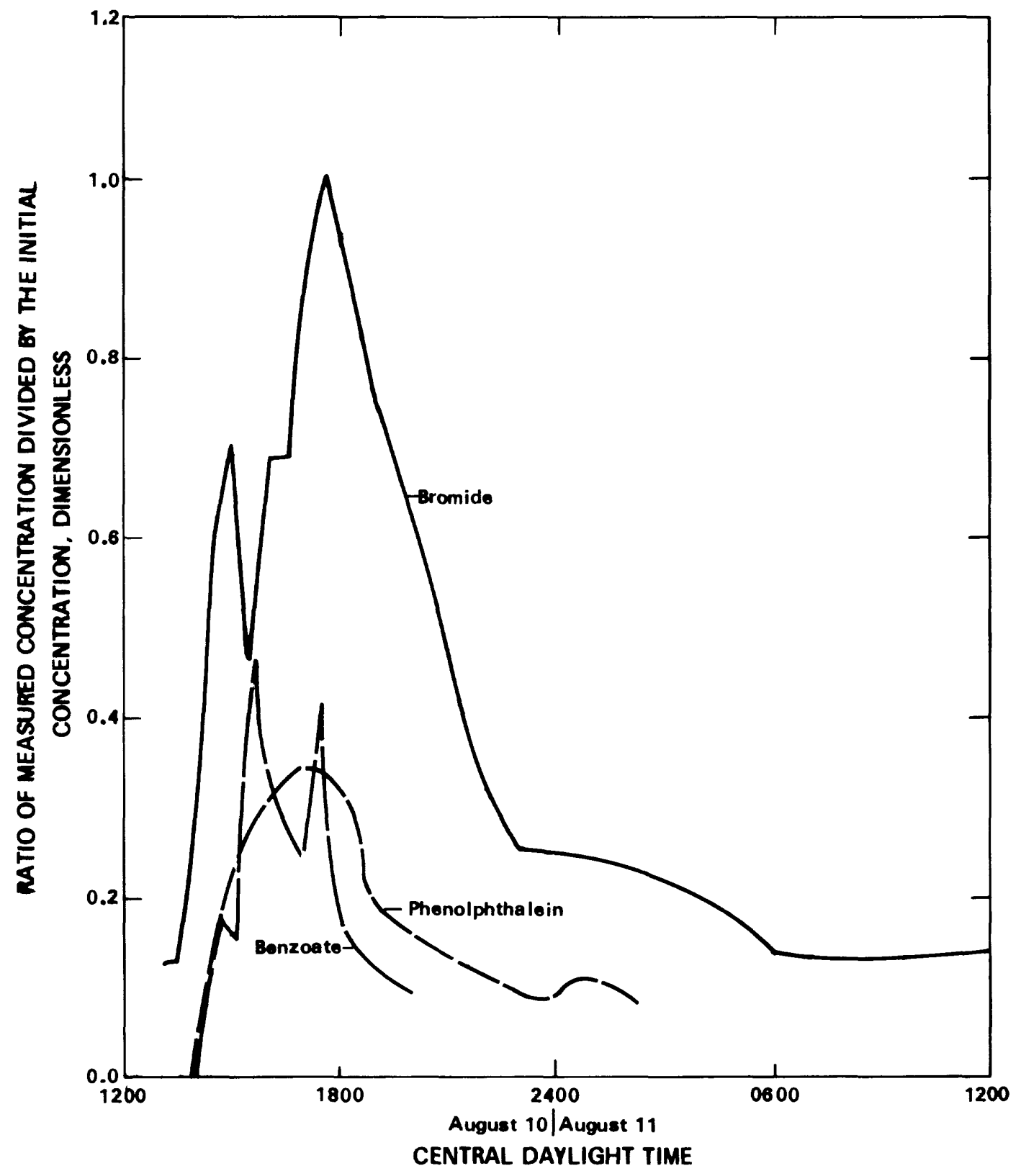

Figure 31.--Comparison of bromide, benzoate, and phenolphthalein data obtained from the 2-meter orange point sampler during the second experiment of the August 1978 test. Concentrations of each tracer have been divided by the input concentration, Co. (Data are from table 63.) 


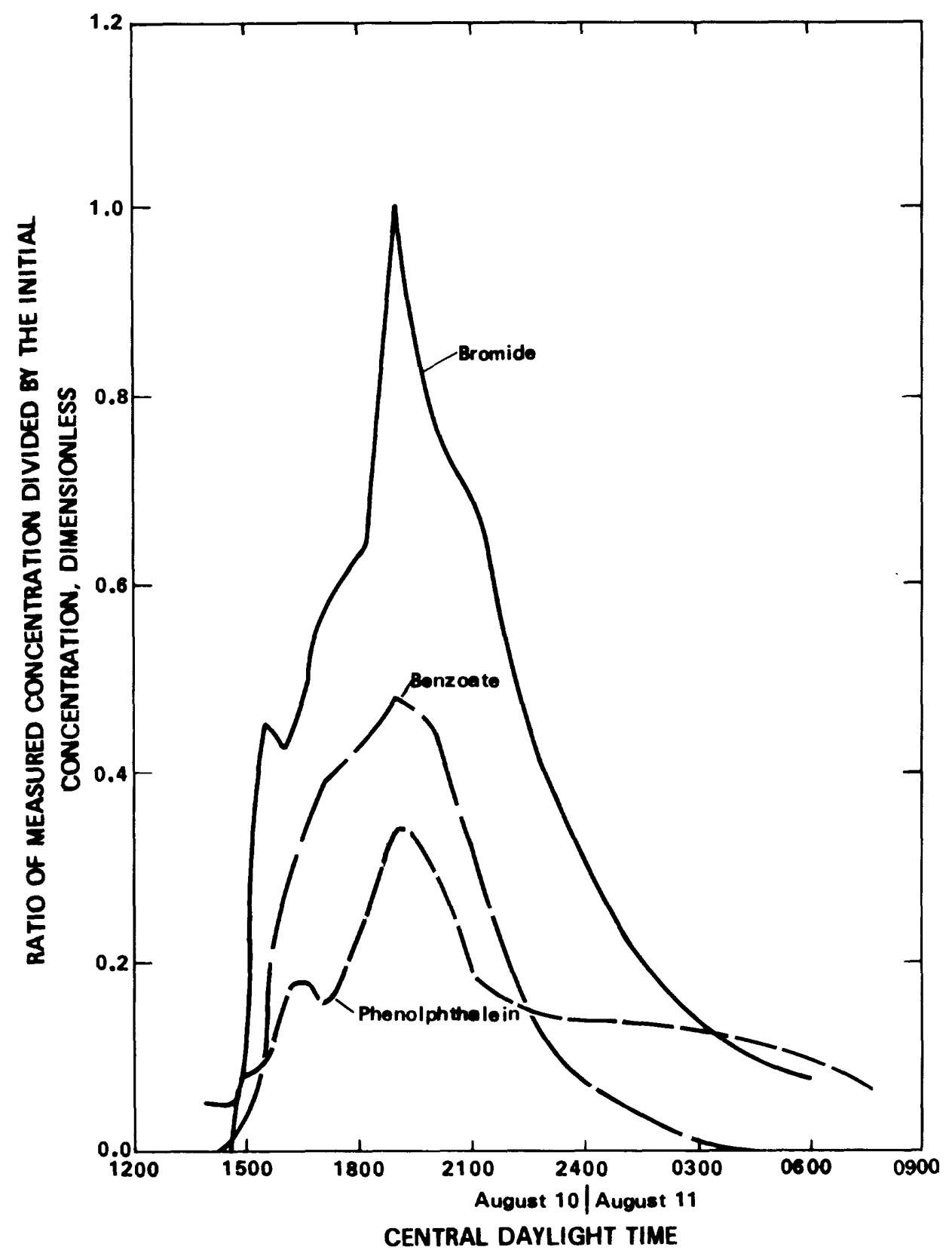

Figure 32.--Comparison of tracer data from the 5-meter orange point sampler during the second experiment of the August 1978 test. Concentrations of each tracer have been divided by the input concentration, Co. (Data are from table 65.) 


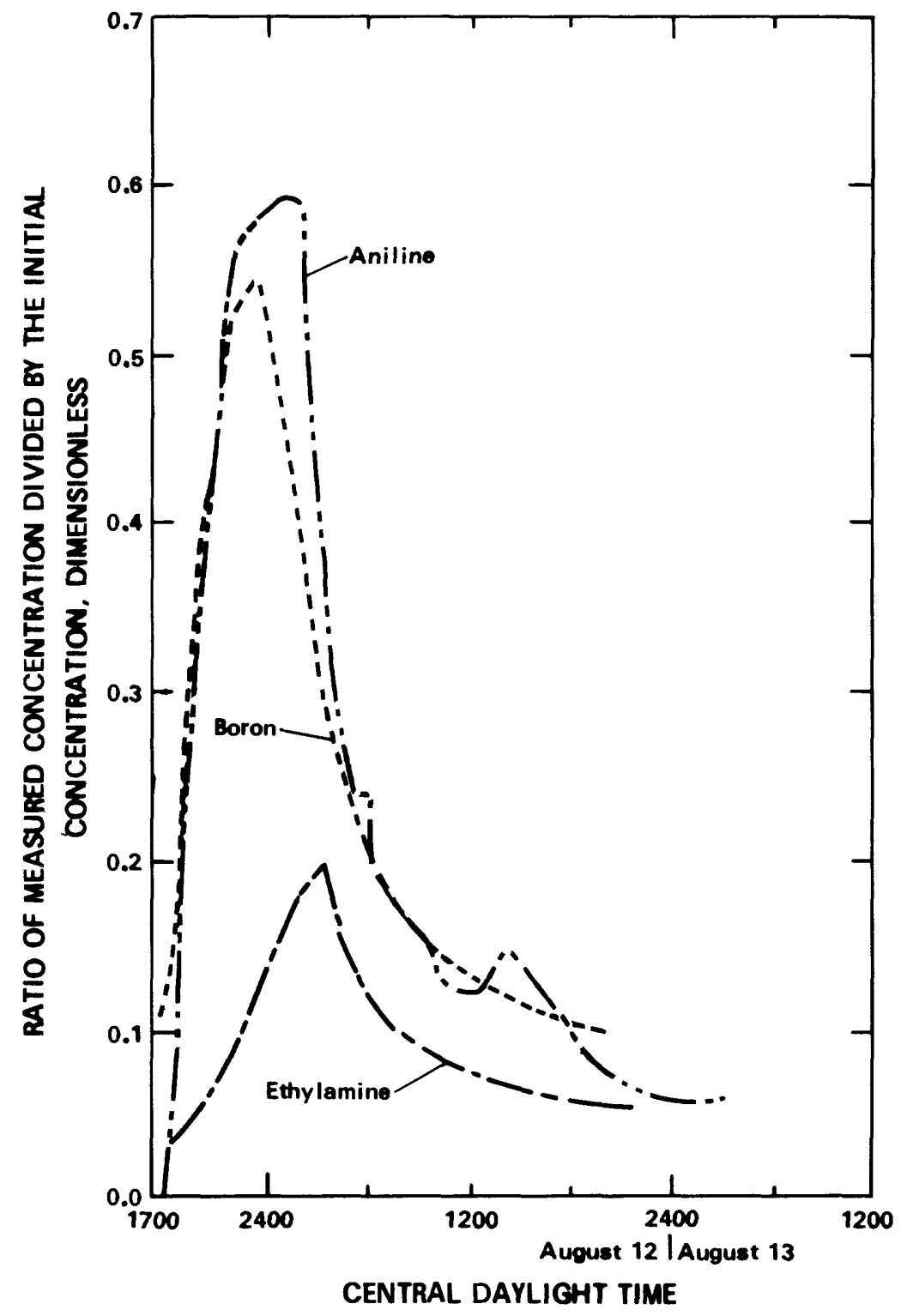

Figure 33.--Comparison of tracer data from the 2-meter orange point sampler during the third experiment of the August 1978 test. Concentrations of each tracer have been divided by the input concentration, Co. (Data are from table 69.) 


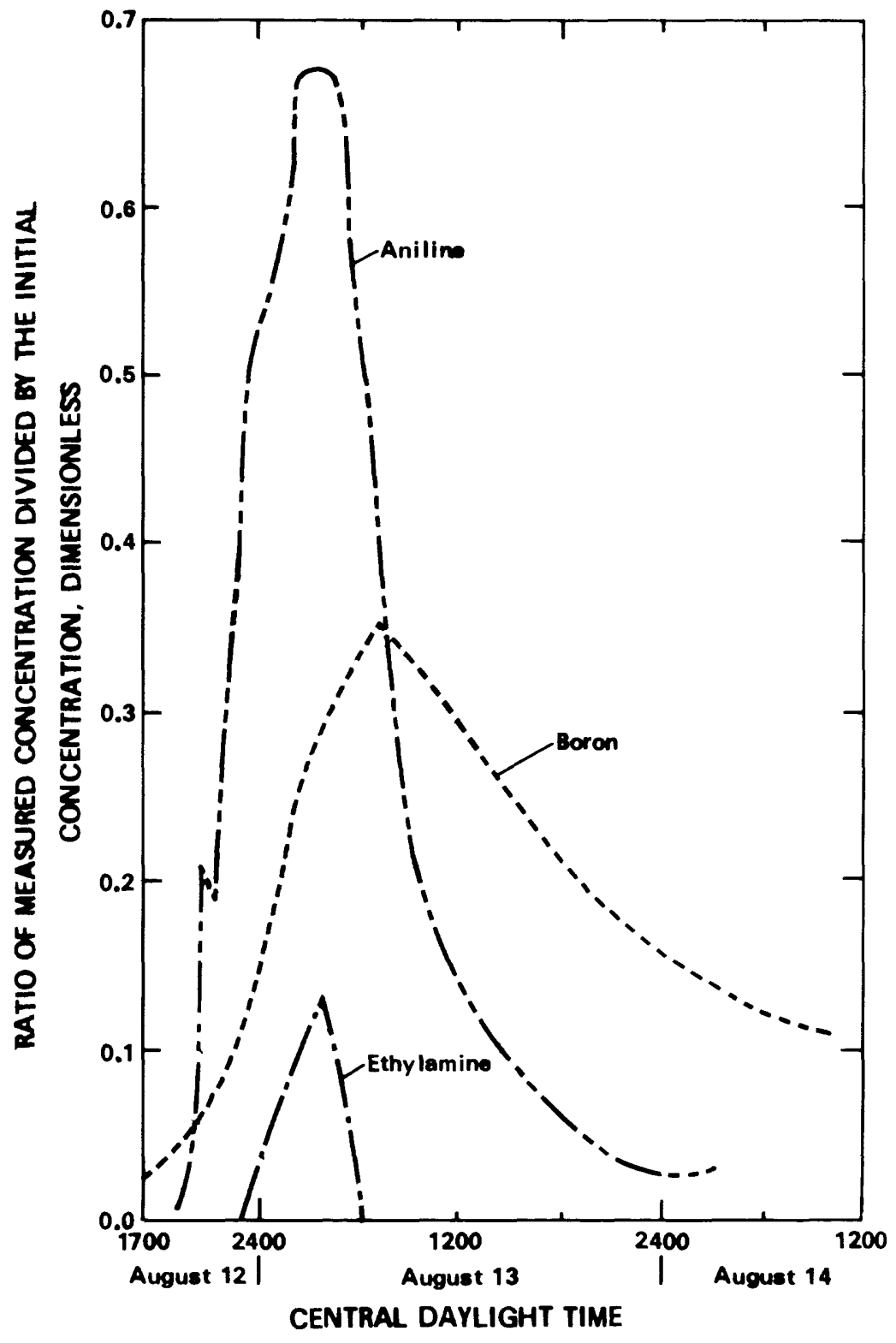

Figure 34.--Comparison of tracer data from the 5-meter orange point sampler during the third experiment of the August 1978 test. Concentrations of each tracer have been divided by the input concentration, Co. (Data are from table 72.) 


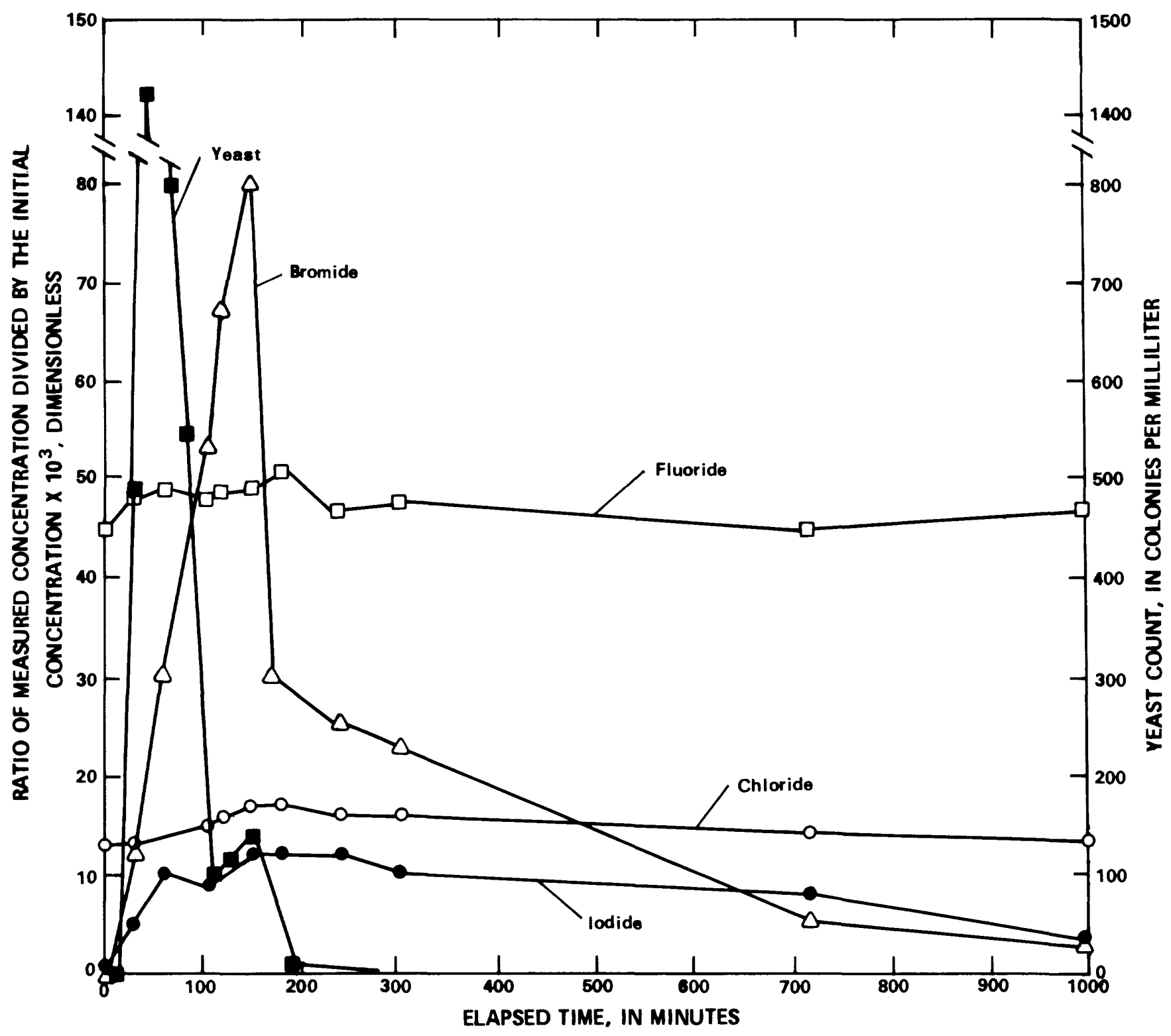

Figure 35.--Comparison of halide tracer data with yeast from the 2-meter well during the fourth experiment (pulse injection) of the August 1978 test. Concentrations of each halide tracer have been divided by the input concentration, Co. (Data are from table 75.) 


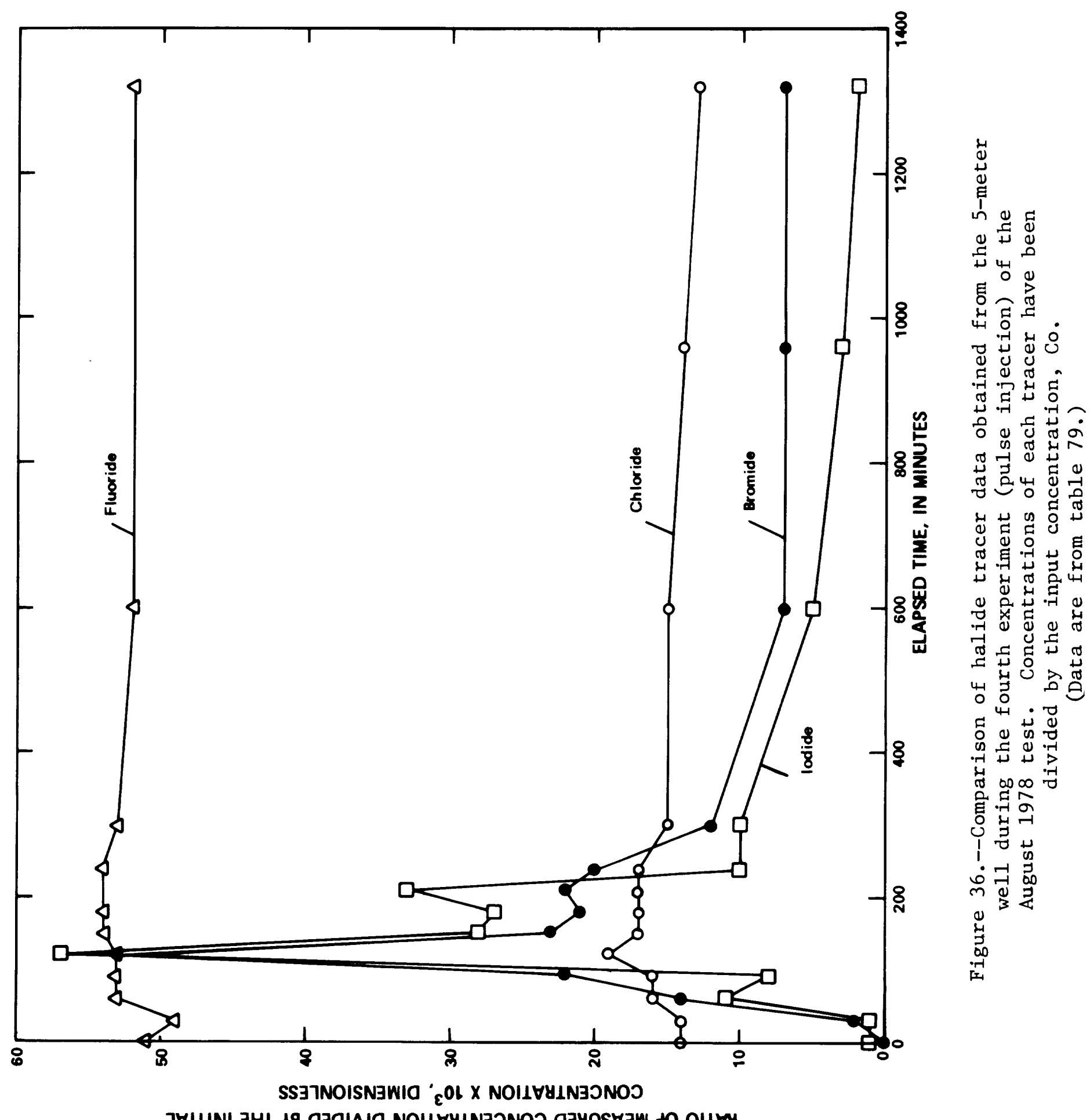

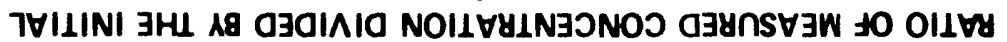


y

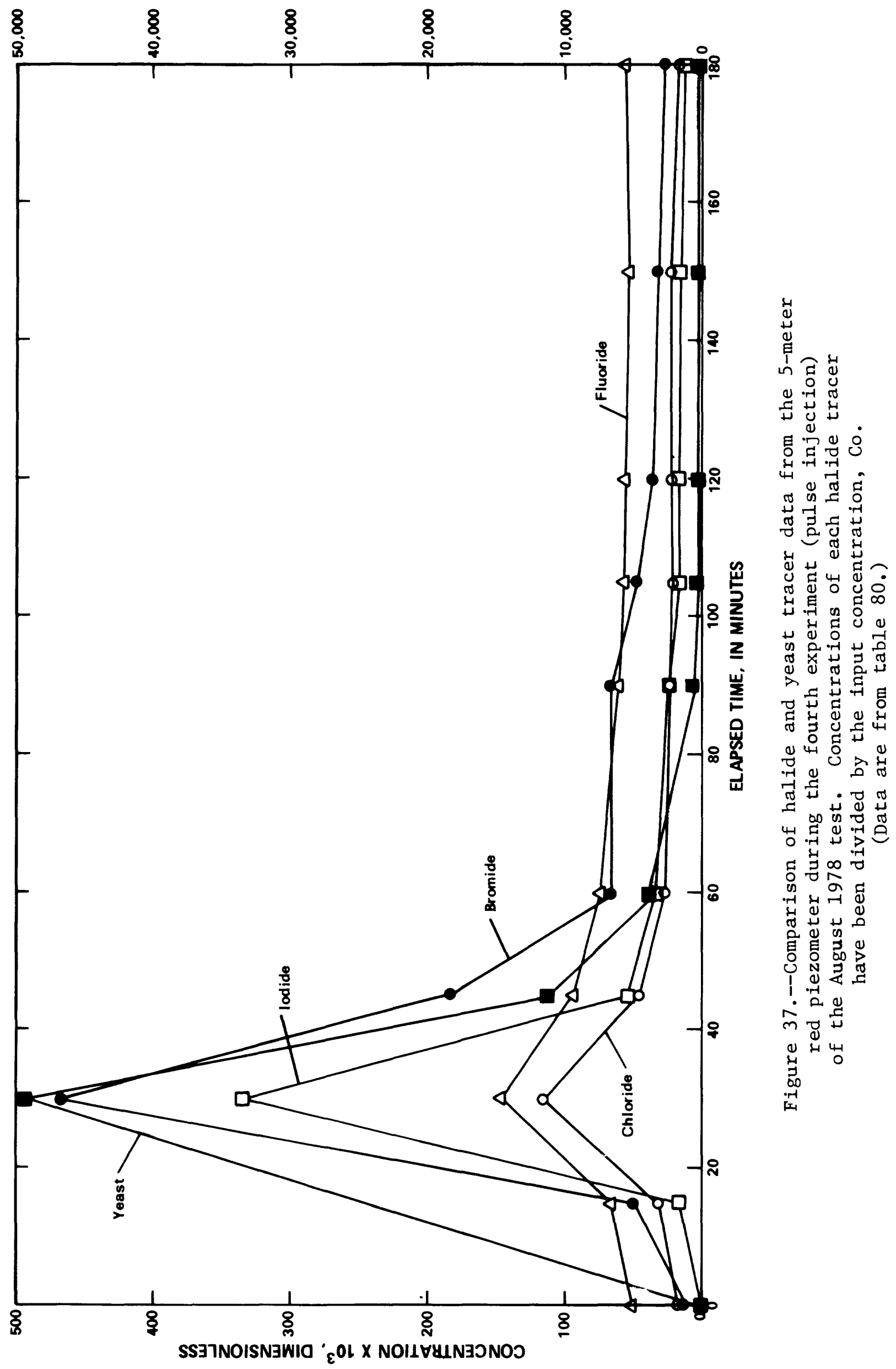

7 


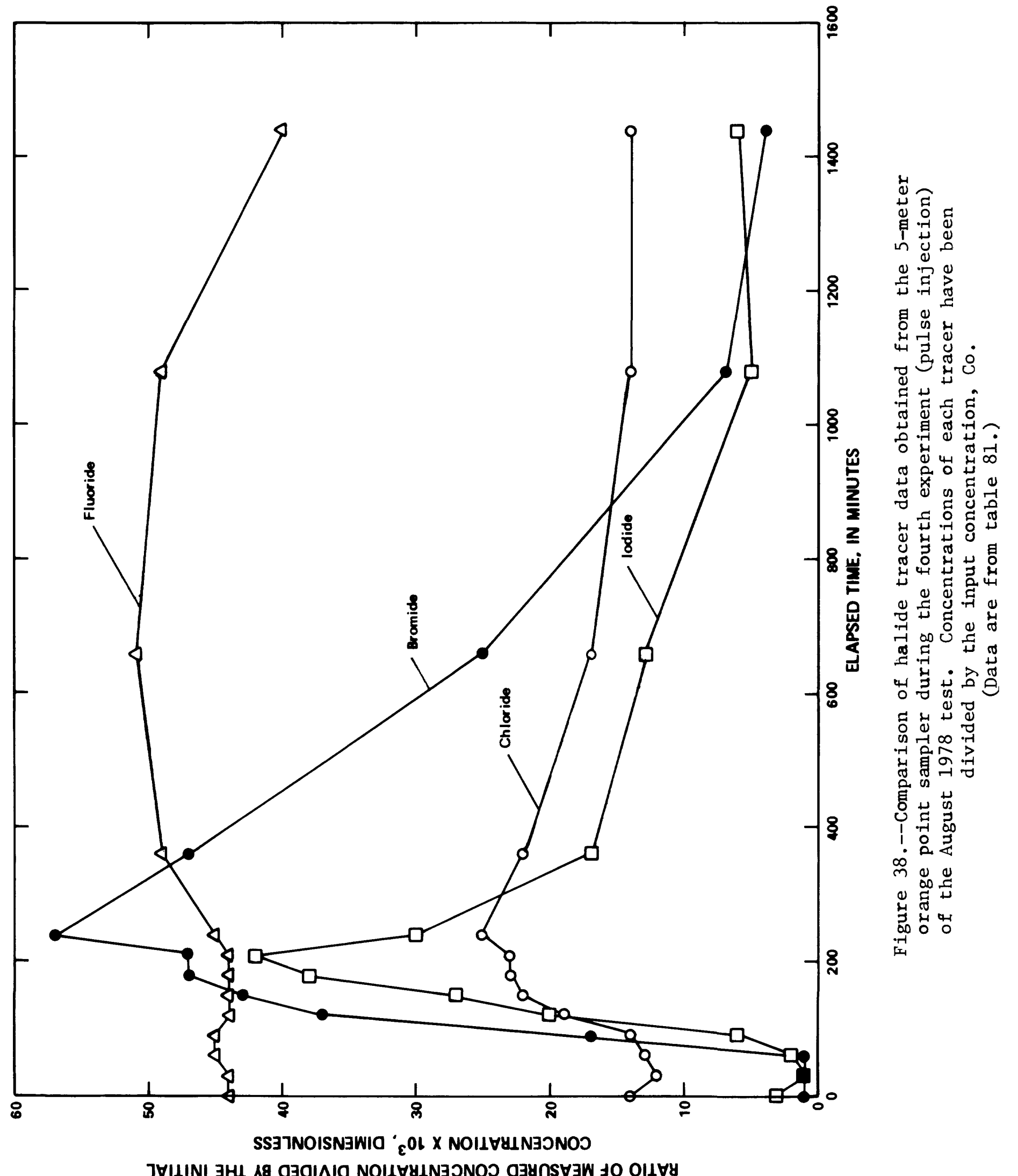




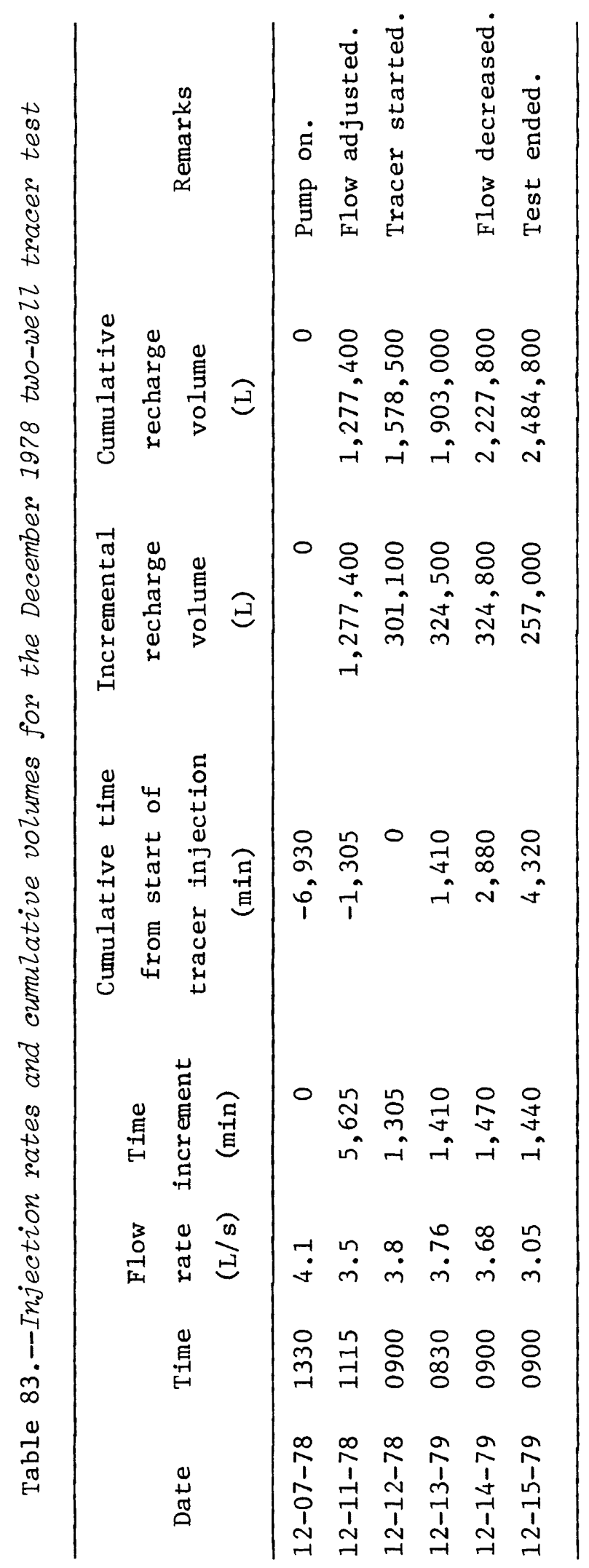


picked up entrained air downstream from the valve, which then plugged the near-well-bore materials in the injection well. An attempt was made to alleviate this problem by switching back-pressure control from one valve to another, to little or no avail.

Two tracers were used for the test, sodium benzoate and boric acid, added at concentrations of $10.0 \mathrm{mg} / \mathrm{L}$ dissolved carbon and $20 \mathrm{mg} / \mathrm{L}$ as boron, respectively. Samples were collected hourly from the injection line upstream from the point at which tracer was introduced, using an automatic sampler. The samples were collected in bottles packed in ice to retard biodegradation of the benzoate, and were re-packed in ice prior to shipment to the laboratory. Measured concentrations of benzoate as dissolved carbon in the injection stream, and in the water pumped from wel1 1 , are 1 isted in table 84. Unfortunately, the samples were inadvertently discarded before the boric-acid analyses could be made.

A11 samples collected after 1600 on December 13, 1978, showed no dissolved carbon. These samples were sent to the laboratory in a second shipment that was delayed in transit. The ice in which they were packed melted, and the samples became warm. Hence, any benzoate present may have biodegraded. 
Table 84.--Chemical data from the two-well tracer test conducted during December 2978 at the Stanton, Tex. site [* Indicates sample not analyzed]

\begin{tabular}{|c|c|c|c|c|}
\hline \multirow{2}{*}{ Date } & \multirow{2}{*}{ Time } & \multirow{2}{*}{$\begin{array}{c}\text { Time since start } \\
\text { of injection } \\
\text { (min) }\end{array}$} & \multicolumn{2}{|c|}{$\begin{array}{c}\text { Benzoate as } \mathrm{C} \\
(\mathrm{mg} / \mathrm{L})\end{array}$} \\
\hline & & & $\begin{array}{c}\text { Injection } \\
\text { sample }\end{array}$ & $\begin{array}{c}\text { Withdrawal } \\
\text { sample }\end{array}$ \\
\hline $12-12-78$ & 1000 & 60 & 9.8 & 0 \\
\hline Do. & 1100 & 120 & $*$ & 0 \\
\hline Do. & 1200 & 180 & $*$ & 0 \\
\hline Do. & 1300 & 240 & $*$ & 0 \\
\hline Do. & 1400 & 300 & 9.9 & 0 \\
\hline Do. & 1500 & 360 & $*$ & .09 \\
\hline Do. & 1600 & 420 & 10.0 & .13 \\
\hline Do. & 1700 & 480 & 10.0 & .17 \\
\hline Do. & 1800 & 540 & 8.8 & .34 \\
\hline Do. & 1900 & 600 & 8.7 & .51 \\
\hline Do. & 2000 & 660 & 10.0 & .8 \\
\hline Do. & 2100 & 720 & 10.2 & $*$ \\
\hline Do. & 2155 & 775 & ---- & 1.0 \\
\hline Do. & 2250 & 830 & --- & 1.0 \\
\hline Do. & 2345 & 885 & ---- & 1.20 \\
\hline $12-13-78$ & 0040 & 940 & --- & 1.11 \\
\hline Do. & 0135 & 995 & --- & 1.36 \\
\hline Do. & 0230 & 1,050 & $\cdots$ & 1.28 \\
\hline Do. & 0325 & 1,105 & $-\cdots$ & .77 \\
\hline Do. & 0420 & 1,160 & ---- & 1.2 \\
\hline Do. & 0610 & 1,270 & ---- & .77 \\
\hline Do. & 0700 & 1,320 & --- & .85 \\
\hline Do. & 0800 & 1,380 & --- & .00 \\
\hline Do. & 0900 & 1,440 & --- & .17 \\
\hline
\end{tabular}


Table 84.--Chemical data from the two-well tracer test conducted during December 1978 at the Stanton, Tex. site--Continued

\begin{tabular}{|c|c|c|c|c|}
\hline \multirow{2}{*}{ Date } & \multirow{2}{*}{ Time } & \multirow{2}{*}{$\begin{array}{c}\text { Time since start } \\
\text { of injection } \\
\text { (min) }\end{array}$} & \multicolumn{2}{|c|}{$\begin{array}{c}\text { Benzoate as } \mathrm{C} \\
(\mathrm{mg} / \mathrm{L})\end{array}$} \\
\hline & & & $\begin{array}{c}\text { Injection } \\
\text { sample }\end{array}$ & $\begin{array}{c}\text { Withdrawal } \\
\text { sample }\end{array}$ \\
\hline $12-13-78$ & 1000 & 1,500 & --- & 0.43 \\
\hline Do. & 1100 & 1,560 & --- & .34 \\
\hline Do. & 1200 & 1,620 & --- & .26 \\
\hline Do. & 1300 & 1,680 & ---- & .17 \\
\hline Do. & 1600 & 1,860 & --- & .09 \\
\hline
\end{tabular}




\section{REFERENCES}

American Public Health Association, American Water Works Association, and Water Pollution Control Federation, 1971, Standard methods for the examination of water and wastewater: New York, American Public Health Association (13th ed.), 874 p.

Brown, R. F., Signor, D. C., and Wood, W. W., 1978, Artificial ground-water recharge as a water management technique on the Southern High Plains of Texas and New Mexico: Texas Department of Water Resources, Report 220, $84 \mathrm{p}$.

Brown, Eugene, Skougstad, M. W., and Fishman, M. J., 1970, Methods for collection and analysis of water samples for dissolved minerals and gases: U.S. Geological Survey Techniques of Water-Resources Investigation, Book 5, Chap. A1, 160 p.

Cooper, H. H., Jr., and Jacob, C. E., 1946, A generalized graphical method for evaluating formation constants and summarizing well-field history: Transactions of the American Geophysical Union, v. 27, no. 4, p. 526-534.

Cronin, J. G., 1964, A summary of the occurrence and development of ground water in the Southern High Plains of Texas: U.S. Geological Survey Water Supply Paper 1693, 88 p.

Davis, S. N., Thompson, G. M., Bentley, H. W., and Stiles, G. K., 1980, Ground-water tracers--A short review: Ground Water, v. 18, p. 14-23.

Hughes, J. L., Eccles, L. A., and Malcolm, R. L., 1974, Dissolved organic carbon (DOC), an index of organic contamination in ground water near Barstow, California: Ground Water, v. 12, p. 283-290.

Leenheer, J. A., Malcolm, R. L., and White, W. R., 1976, Physical, chemical, and biological aspects of subsurface organic waste injection near Wilmington, North Carolina: U.S. Geological Survey Professional Paper 987, 56 p.

Orion Research, Inc., 1977, Instruction manual: halide electrodes: Orion Research Incorporated, form $94 \mathrm{H} / 776,31 \mathrm{p}$.

Signor, D. C., 1978, Gas-driven pump for ground-water samples: U.S. Geological Survey Water Resources Investigations 78 (72), 25 p.

Theis, C. V., 1935, The relation between the lowering of the piezometric surface and the rate and duration of discharge of a well using groundwater storage: Transactions of the American Geophysical Union, pt. 2, p. 519-524.

Thompson, G. M., 1976, Trichlorofluoromethane, a new tool for tracing and dating ground water: $\mathrm{Ph} . \mathrm{D}$. dissertation, Indiana University, Bloomington, Indiana, $93 \mathrm{p}$.

Thompson, G. M., and Hays, J. M., 1979, Trichlorofluoromethane in ground water--a possible tracer and indicator of ground water age: Water Resources Research, v. 15, p. 546-553.

Weeks, E. P., 1969, Determining the ratio of horizontal to vertical permeability by aquifer test analysis: Water Resources Research, v. 5, p. 196-214.

1979, Barometric fluctuations in wells tapping deep unconfined aquifers: Water Resources Research, v. 15, p. 1167-1176. 
Wood, W. W., 1973, A technique using porous cups for water sampling at any depth in the unsaturated zone: Water Resources Research, v. 9, p. 486-488.

, 1976, Guidelines for collection and field analysis of ground water samples for selected unstable constituents: U.S. Geological Survey Techniques of Water-Resources Investigation, Chap. D2, 24 p.

Wood, W. W., and Ehrlich, G. G., 1978, Use of Baker's yeast to trace microbial movement in ground water: Ground Water, v. 16, p. 398-403. 